\title{
Reticular Synthesis of tbo Topology Covalent Organic Frameworks
}

Xing Kang, Xing Han, Chen Yuan, Cheng Cheng, Yan Liu*, and Yong Cui* School of Chemistry and Chemical Engineering, Frontiers Science Center for Transformative Molecules and State Key Laboratory of Metal Matrix Composites, Shanghai Jiao Tong University, Shanghai 200240, China

Email:yongcui@sju.edu.cn; liuy@sjtu.edu.cn.

\section{Table of Content}

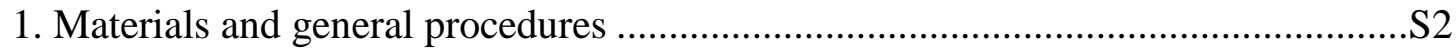

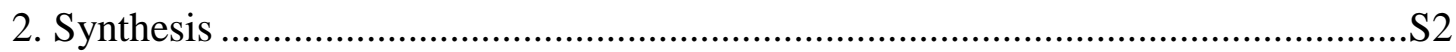

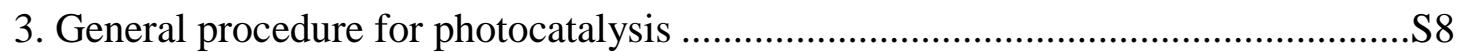

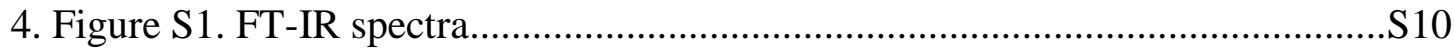

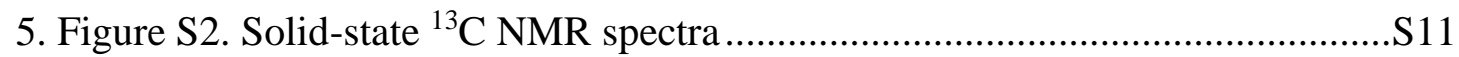

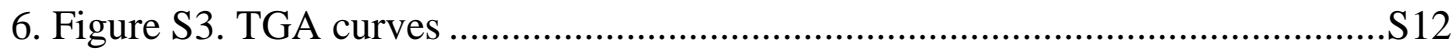

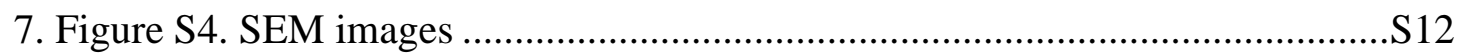

8. Figures S5-S23. Structural modeling and PXRD analysis of the COFs ................S13

9. Tables S1-S16. Fractional atomic coordinates and unit cell parameters ...............S23

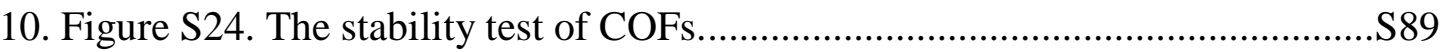

11. Figures S25-S29 \& Table S17. Dye adsorption measurements ..........................S90

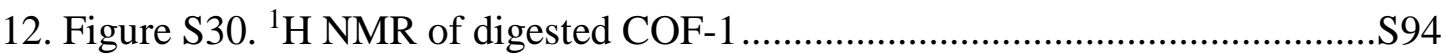

13. Figure S31 Solid-state UV spectra and band gaps of monomers........................S95

14. Figure S32 Normalized absorption and emission spectra of COFs ....................S96

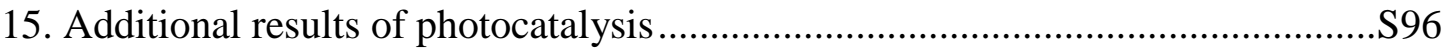

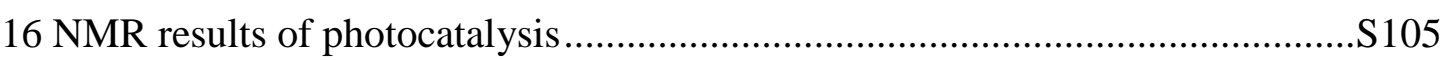

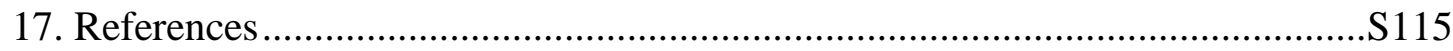




\section{Materials and general procedures}

All the chemicals are commercial available, and used without further purification. The IR (KBr pellet) spectra were recorded (400-4000 $\mathrm{cm}^{-1}$ region) on a Nicolet Magna 750 FT-IR spectrometer. Powder X-ray diffraction data (PXRD) were collected on a D8 Advance diffractometer using $\mathrm{Cu} \mathrm{K} \alpha$ radiation with $1.5418 \AA$ ranging from $0.6002^{\circ}$ to $35.0066^{\circ}$ by $0.2 \mathrm{~s}$ for 1679 steps. Elemental analysis (EA) was performed on Flash Smart (ThermoFisher, China). The confocal fluorescence microscopy images were obtained on a (Leica)/TCS SP8 STED 3X super-resolution multiphoton confocal microscope (SMCM). Thermogravimetric analyses (TGA) were carried out in an air atmosphere with a heating rate of $10{ }^{\circ} \mathrm{C} / \mathrm{min}$ on a STA449C integration thermal analyzer. Scanning Electron Microscopy (SEM) was conducted on Nova NanoSEM 450. ${ }^{1} \mathrm{H}$ NMR experiments were carried out on a MERCURY plus 400 spectrometer operating at resonance frequencies of $400 \mathrm{MHz}$. Solid ${ }^{13} \mathrm{C}$ NMR experiments were carried out on a Bruker Avance III $600 \mathrm{MHz}$. The solid-state UV spectra were recorded by using pressed $\mathrm{BaSO}_{4}$ matrix in the range of 200-800 $\mathrm{nm}$ at room temperature with a UV/Vis/NIR Spectrometer Lambda 750S (Perkin Elmer, Inc., USA). Inductively Coupled Plasma Optical Emission Spectrometer (ICP-OES) was performed on Optima 7300DV ICP-OES (Perkin Elmer Coporation, USA). Electron spin resonance (ESR) spectra were recorded at room temperature using a Bruker ESP-300E spectrometer at 9.8 GHz, X-band, with $100 \mathrm{~Hz}$ field modulation. High-resolution mass (HRMS) data was recorded on an Acquity UPLC / QTOF Premier spectrometer. The Material Studio (MS) software was used to simulate and describe the crystal structures. The ToposPro, Systre and 3dt software were designed to analyze periodic nets of crystal structures. The diamond software was used to construct the structural model.

\section{Synthesis}

\subsection{Synthesis of the monomers}

5,10,15,20-tetra(4-aminophenyl)porphyrin[H $\mathbf{H}_{2}$ (TAP)] and 5,10,15,20-tetrakis[(4aminophenyl)-porphinato]copper(II) $[\mathbf{C u}(\mathbf{T A P})]$ were synthesized according to the published procedures. ${ }^{\mathbf{1 , 2}}$ 


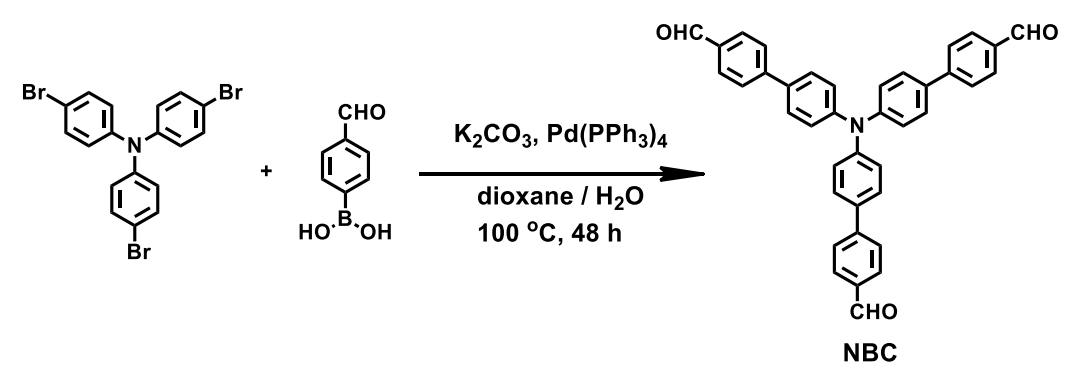

A mixture of tris(4-bromophenyl)amine (4.8 g, $10 \mathrm{mmol})$, 4-formylphenylboronic acid (5.4 g, $36 \mathrm{mmol}), \mathrm{K}_{2} \mathrm{CO}_{3}(11.0 \mathrm{~g}, 80 \mathrm{mmol})$ and $\mathrm{Pd}\left(\mathrm{PPh}_{3}\right)_{4}(570 \mathrm{mg}, 0.5 \mathrm{mmol})$ in dioxane $/ \mathrm{H}_{2} \mathrm{O}(19 / 1 \mathrm{v} / \mathrm{v}, 200 \mathrm{~mL})$ was degassed for $10 \mathrm{~min}$. The suspension was stirred under $\mathrm{N}_{2}$ at $100{ }^{\circ} \mathrm{C}$ for $48 \mathrm{~h}$. After cooling to room temperature, the mixture was concentrated and then extracted with dichloromethane (DCM). The organic phase was washed with brine, dried over anhydrous $\mathrm{Na}_{2} \mathrm{SO}_{4}$ and then concentrated under reduced pressure. The crude product was purified by silica gel column chromatography (petroleum ether/DCM, 1:2, v/v) to afford 4',4"',4"'"'-nitrilotris[(1,1'-biphenyl) 4carbaldehyde] (NBC) $(3.6 \mathrm{~g}, 65 \%) .{ }^{1} \mathrm{H} \mathrm{NMR}\left(400 \mathrm{MHz}, \mathrm{CDCl}_{3}\right) \delta 10.05$ (s, 3H), 7.96 $-7.94(\mathrm{~d}, J=8.2 \mathrm{~Hz}, 8 \mathrm{H}), 7.77-7.75(\mathrm{~d}, J=8.2 \mathrm{~Hz}, 8 \mathrm{H}), 7.62-7.60(\mathrm{~d}, J=8.6 \mathrm{~Hz}, 8 \mathrm{H})$, $7.29-7.27(\mathrm{~d}, J=8.6 \mathrm{~Hz}, 8 \mathrm{H})$.

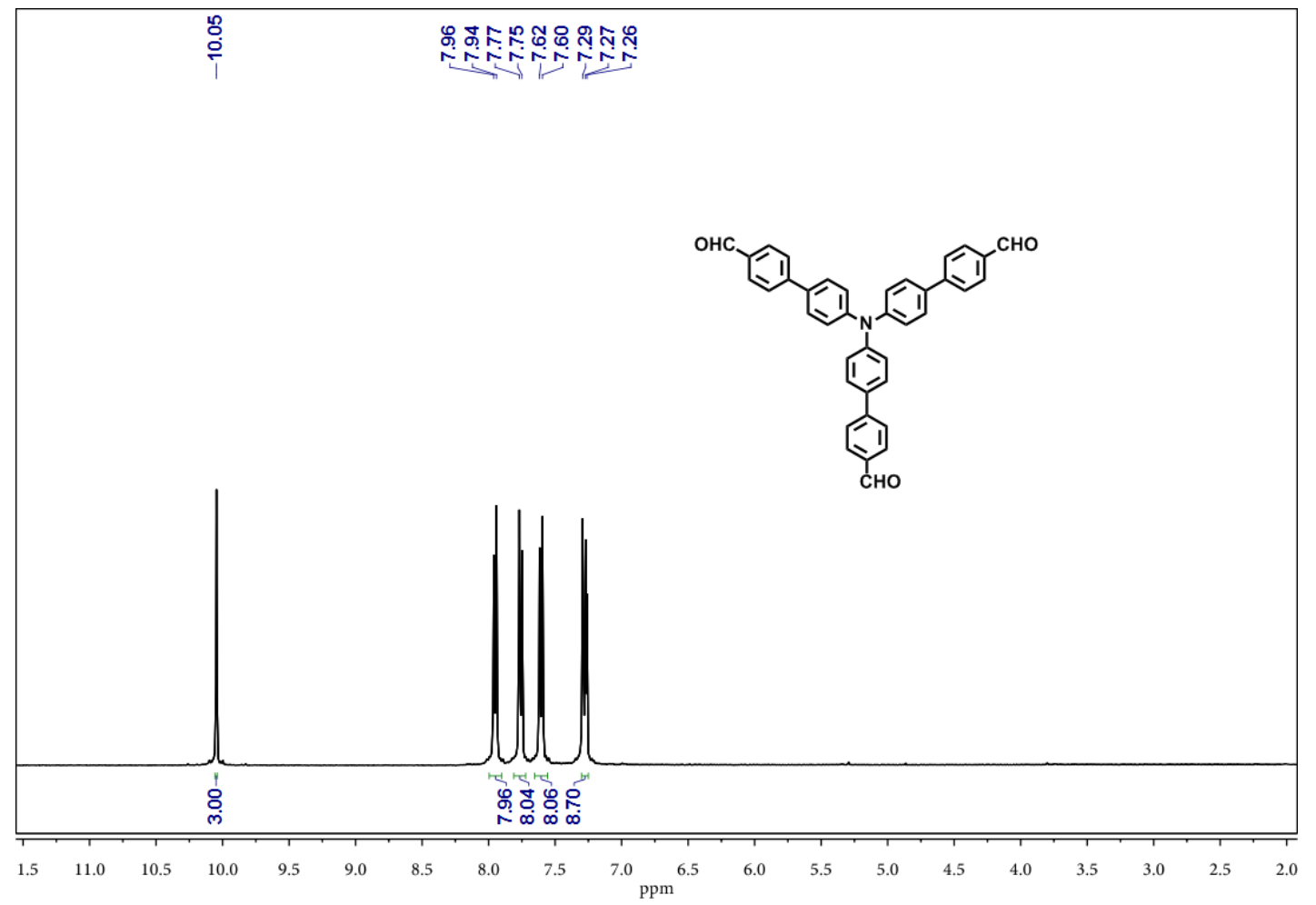




\subsection{Synthesis of COFs}

Synthesis of COF-1. A mixture of NBC (20 mg, $0.036 \mathrm{mmol}$ ), H2(TAP) (18.1 mg, $0.027 \mathrm{mmol}), n$-BuOH $(0.3 \mathrm{~mL})$ and o-dichlorobenzene $(o$-DCB) $(1.2 \mathrm{~mL})$ in a small vial was sonicated for $5 \mathrm{~min}$. The aqueous acetic acid $(6 \mathrm{M}, 0.2 \mathrm{~mL})$ was added, and the mixture was sonicated to afford a homogeneous dispersion. The solution was transferred into a pyrex tube and degassed by three freeze-pump-thaw cycles. The tube was sealed off and heated at $120{ }^{\circ} \mathrm{C}$ for $5 \mathrm{~d}$. The solid was collected by filtration and washed with anhydrous DMF and acetone. The powder was dried at $60{ }^{\circ} \mathrm{C}$ under vacuum overnight to afford COF-1 as a black purple solid (30.4 mg, 80\%). Elemental Analysis of COF-1: Anal(\%). Calcd. for $\mathrm{C}_{144} \mathrm{H}_{90} \mathrm{~N}_{14} \cdot 3\left(\mathrm{H}_{2} \mathrm{O}\right)$ : C, 83.56; H, 4.64; N, 9.48. Found: C, 82.13; H, 4.94; N, 9.30.

Synthesis of COF-2. COF-2 was synthesized in a similar procedure by using Cu(TAP) (19.8 mg, $0.027 \mathrm{mmol}$ ). The powder was washed with DMF and acetone, and then dried at $60{ }^{\circ} \mathrm{C}$ under vacuum overnight to afford COF-2 as a red solid (29.8 mg, 75\%). Elemental Analysis of COF-2: $\mathrm{Anal}(\%)$. Calcd. for $\mathrm{C}_{288} \mathrm{H}_{180} \mathrm{~N}_{28} \mathrm{Cu}_{3} \cdot 30\left(\mathrm{H}_{2} \mathrm{O}\right)$ : C, 72.57; H, 5.04; N, 8.24. Found: C, 71.76; H, 4.81; N, 8.30. ICP-OES of COF-2: Cu, $4.169 \mathrm{wt} \%$.

\subsection{Synthesis of dye molecules}
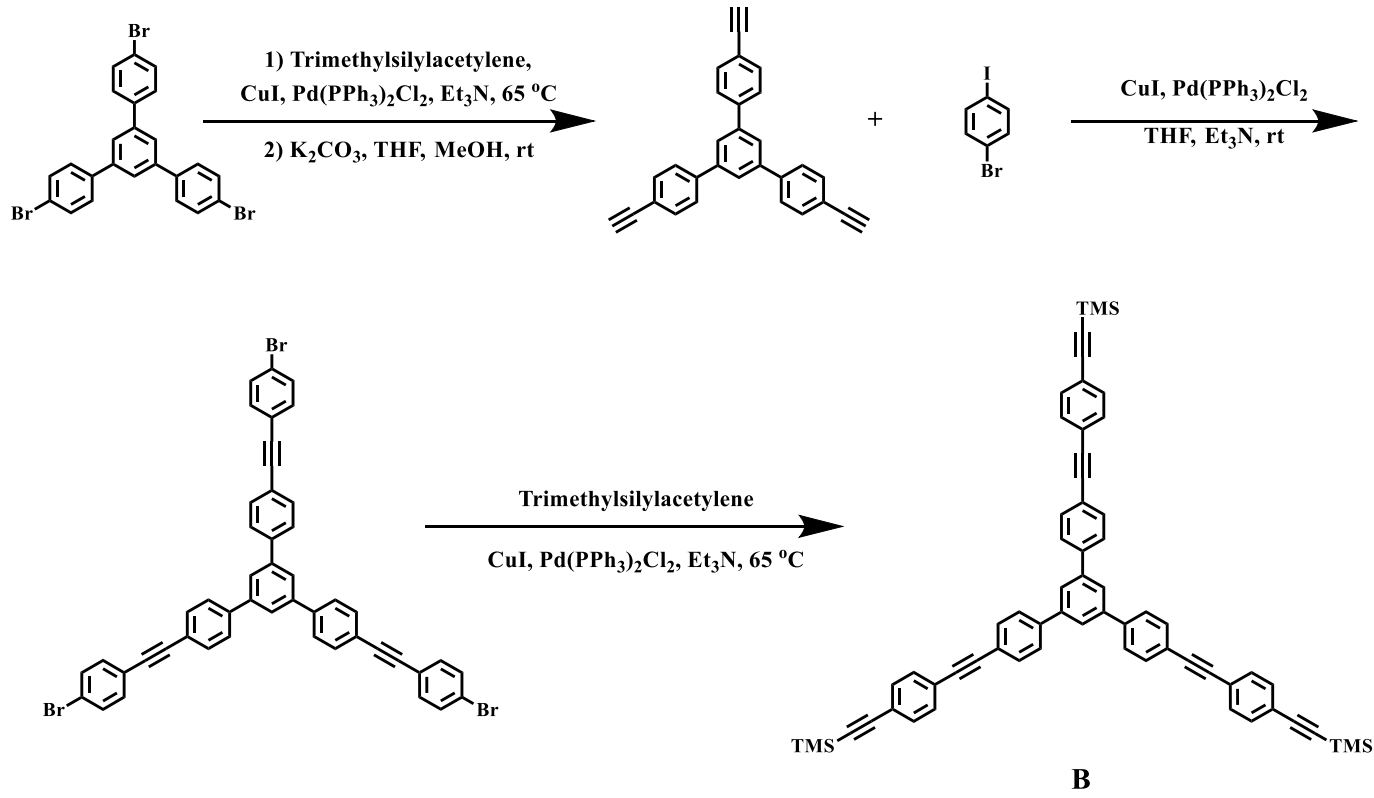
1,3,5-tris[4'-(ethynyl)phenyl]benzene. 1,3,5-tris(4-bromophenyl)benzene (10.0 g, $18.6 \mathrm{mmol}), \mathrm{Pd}\left(\mathrm{PPh}_{3}\right)_{2} \mathrm{Cl}_{2}(0.647 \mathrm{~g}, 0.92 \mathrm{mmol})$ and $\mathrm{CuI}(87.6 \mathrm{mg}, 0.46 \mathrm{mmol})$ were dissolved in $\mathrm{Et}_{3} \mathrm{~N}(150 \mathrm{~mL})$. The mixture was purged with $\mathrm{N}_{2}$ for $10 \mathrm{~min}$, and then trimethylsilylacetylene $(15.4 \mathrm{~g}, 157 \mathrm{mmol})$ was added. The suspension was stirred under $\mathrm{N}_{2}$ at $65{ }^{\circ} \mathrm{C}$ for $16 \mathrm{~h}$. After cooling to room temperature, the mixture was concentrated and then extracted with dichloromethane (DCM). The organic phase was washed with brine, dried over anhydrous $\mathrm{Na}_{2} \mathrm{SO}_{4}$, and then concentrated under reduced pressure. The crude product was purified by column chromatography on silica gel (petroleum ether/EtOAc, 20:1, v/v) to afford 1,3,5-trid\{4'-[(trimethylsiyl) ethynynl]phenyl $\}$ benzene. The obtained 1,3,5-trid $\{4$ '-[(trimethylsiyl)ethynynl]phenyl $\}$ benzene $(6.0 \mathrm{~g}, 10 \mathrm{mmol})$ was dissoved in the mixture of THF/MeOH $(100 \mathrm{~mL}, 1: 4$, $\mathrm{v} / \mathrm{v}) . \mathrm{K}_{2} \mathrm{CO}_{3}(8.3 \mathrm{~g}, 60 \mathrm{mmol})$ was added, and the reaction mixture was stirred at room temperature for $24 \mathrm{~h}$. The mixture was filtered, and the organic phase was collected and concentrated under reduced pressure. The crude product was purified by column chromatography on silica gel (petroleum ether/DCM, 10:1, v/v) to afford 1,3,5-tris[4'(ethynyl)phenyl)-benzene (2.8 g, $75 \%$ ). ${ }^{1} \mathrm{H}$ NMR (400 MHz, $\left.\mathrm{CDCl}_{3}\right) \delta 7.76(\mathrm{~s}, 3 \mathrm{H})$, 7.66-7.60 (m, 12H), 3.17 (s, 3H).

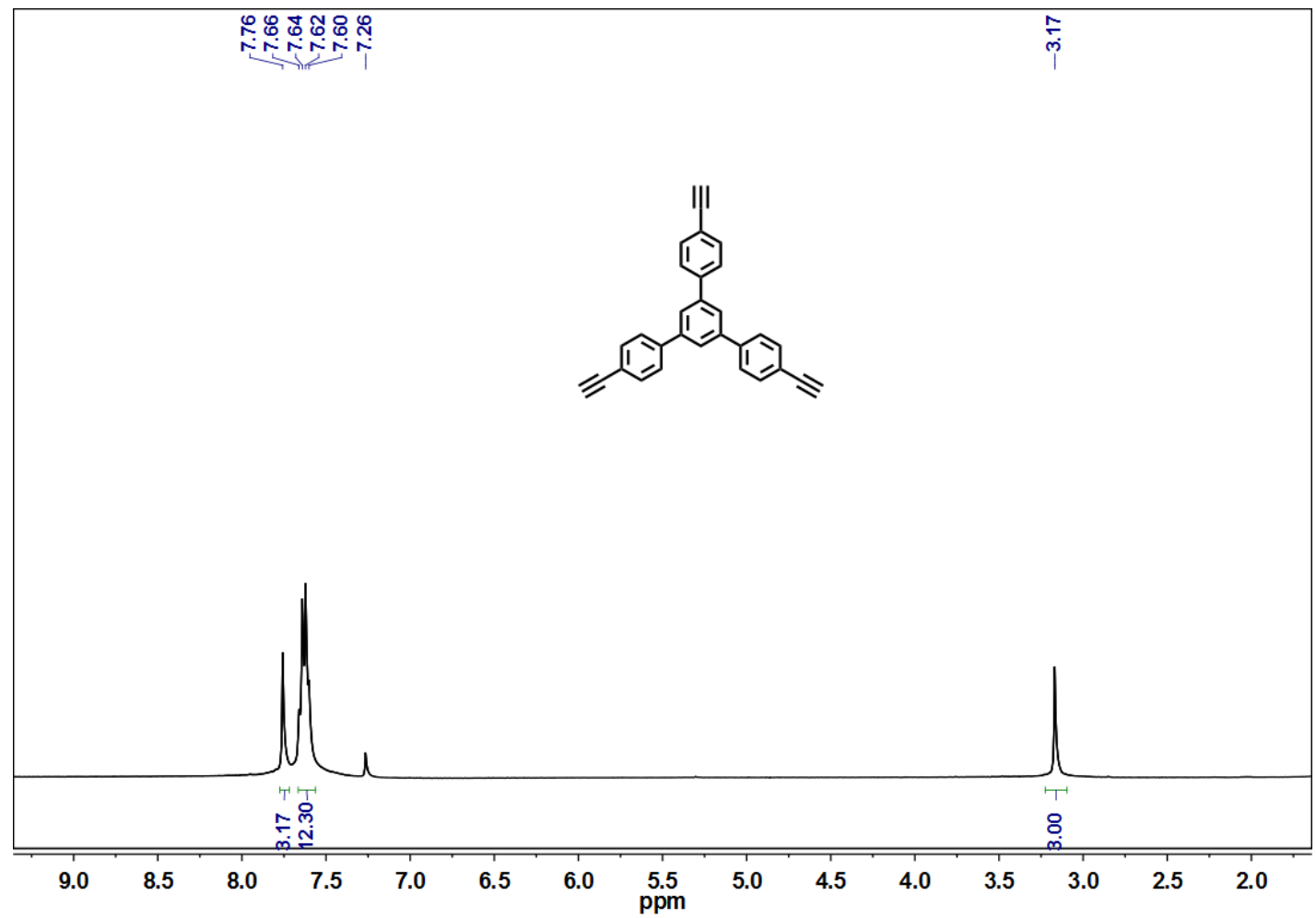


1,3,5-tris $\left\{4^{\prime}\left[\left(4^{\prime \prime}\right.\right.\right.$-bromophenyl)ethynyl]phenyl $\}$ benzene. A mixture of 1,3,5tris[4'-(ethynyl) phenyl)-benzene( $2 \mathrm{~g}, 5.3 \mathrm{mmol}), \mathrm{Pd}\left(\mathrm{PPh}_{3}\right)_{2} \mathrm{Cl}_{2}(185 \mathrm{mg}, 0.26 \mathrm{mmol})$, $\mathrm{CuI}(80 \mathrm{mg}, 0.42 \mathrm{mmol})$ and 4-bromo-iodobenzen $(6.7 \mathrm{~g}, 23.85 \mathrm{mmol}) \mathrm{in}^{\mathrm{E}} \mathrm{Et}_{3} \mathrm{~N} / \mathrm{THF}$ $(2 / 3 \mathrm{v} / \mathrm{v}, 50 \mathrm{~mL})$ was purged with $\mathrm{N}_{2}$ for $10 \mathrm{~min}$. The mixture was stirred at room temperature for $24 \mathrm{~h}$ under $\mathrm{N}_{2}$ atmosphere. The mixture was then filtered through suction funnel. The solid was washed with water, $\mathrm{MeOH}$ and diethyl ether, and dried at $60{ }^{\circ} \mathrm{C}$ under vacuum overnight to afford the target product as a yellow solid $(2.9 \mathrm{~g}$, $65 \%) .{ }^{1} \mathrm{H}$ NMR (400 MHz, $\left.\mathrm{CDCl}_{3}\right) \delta 7.80(\mathrm{~s}, 3 \mathrm{H}), 7.71$-7.63 (m, 12H), 7.51-7.41 (m, $12 \mathrm{H})$.

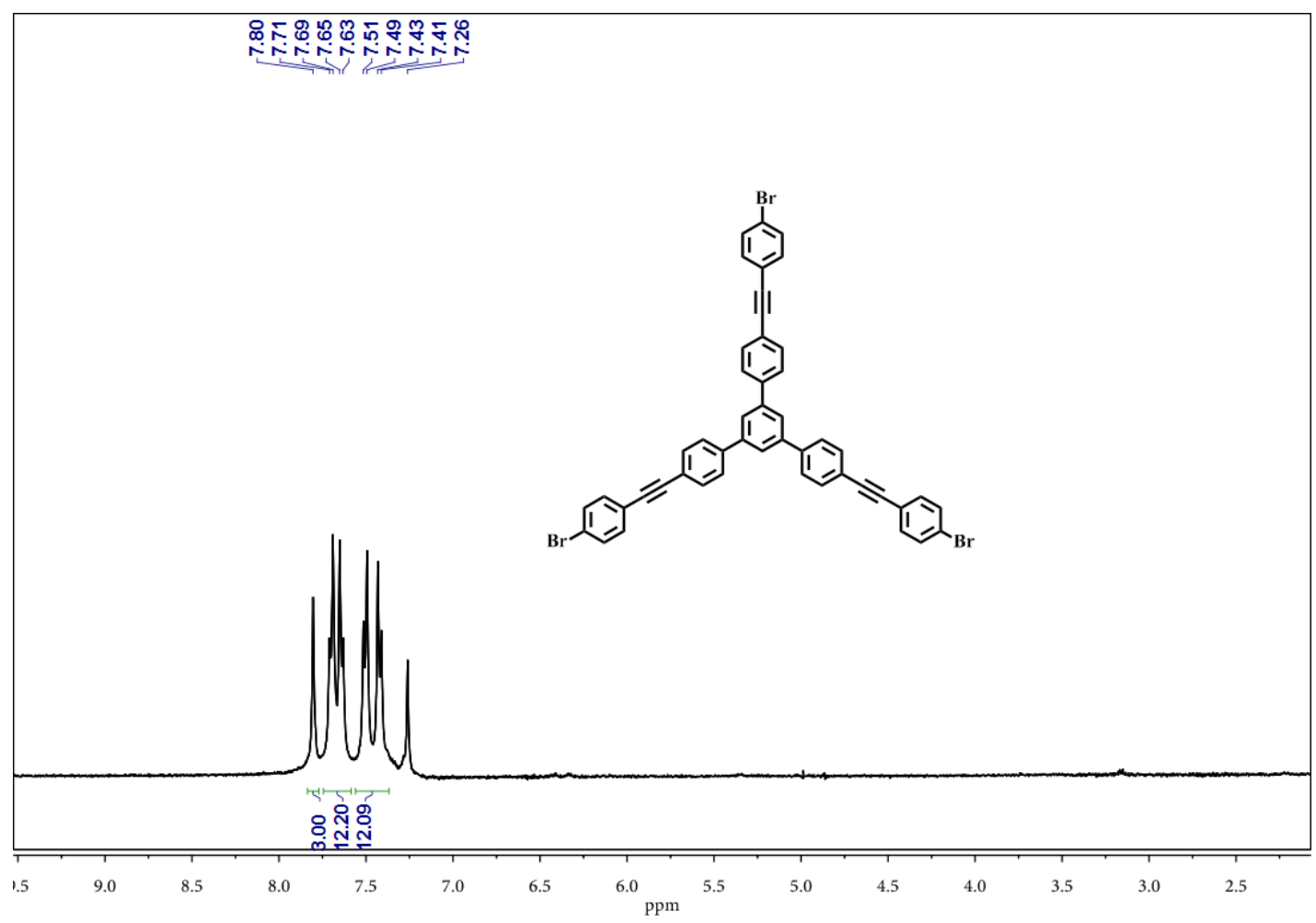

Compound B. 1,3,5-tris\{4'[(4"-bromophenyl)-ethynyl]phenyl) benzene (1.54 g, $1.84 \mathrm{mmol}), \mathrm{Pd}\left(\mathrm{PPh}_{3}\right)_{2} \mathrm{Cl}_{2}(64.7 \mathrm{mg}, 0.092 \mathrm{mmol})$ and $\mathrm{CuI}(8.76 \mathrm{mg}, 0.046 \mathrm{mmol})$ were dissolved in $\mathrm{Et}_{3} \mathrm{~N}$ (30 mL). The mixture was purged with $\mathrm{N}_{2}$ for $10 \mathrm{~min}$, and then trimethylsilylacetylene $(1.26 \mathrm{~g}, 12.9 \mathrm{mmol})$ was added. The suspension was stirred under $\mathrm{N}_{2}$ at $65{ }^{\circ} \mathrm{C}$ for $24 \mathrm{~h}$. After cooling to room temperature, the mixture was concentrated and then extracted with dichloromethane (DCM). The organic phase was washed with brine, dried over anhydrous $\mathrm{Na}_{2} \mathrm{SO}_{4}$, and then concentrated under reduced pressure. The crude product was purified by column chromatography on silica gel (petroleum ether/EtOAc, 10:1, v/v) to afford $\mathbf{B}$ as a yellow solid $(1.15 \mathrm{~g}, 70 \%) .{ }^{1} \mathrm{H}$ 
NMR (400 MHz, $\left.\mathrm{CDCl}_{3}\right) \delta 7.79(\mathrm{~s}, 3 \mathrm{H}), 7.70-7.63(\mathrm{~m}, 12 \mathrm{H}), 7.51-7.39(\mathrm{~m}, 12 \mathrm{H}), 0.27$ $(\mathrm{s}, 27 \mathrm{H})$.
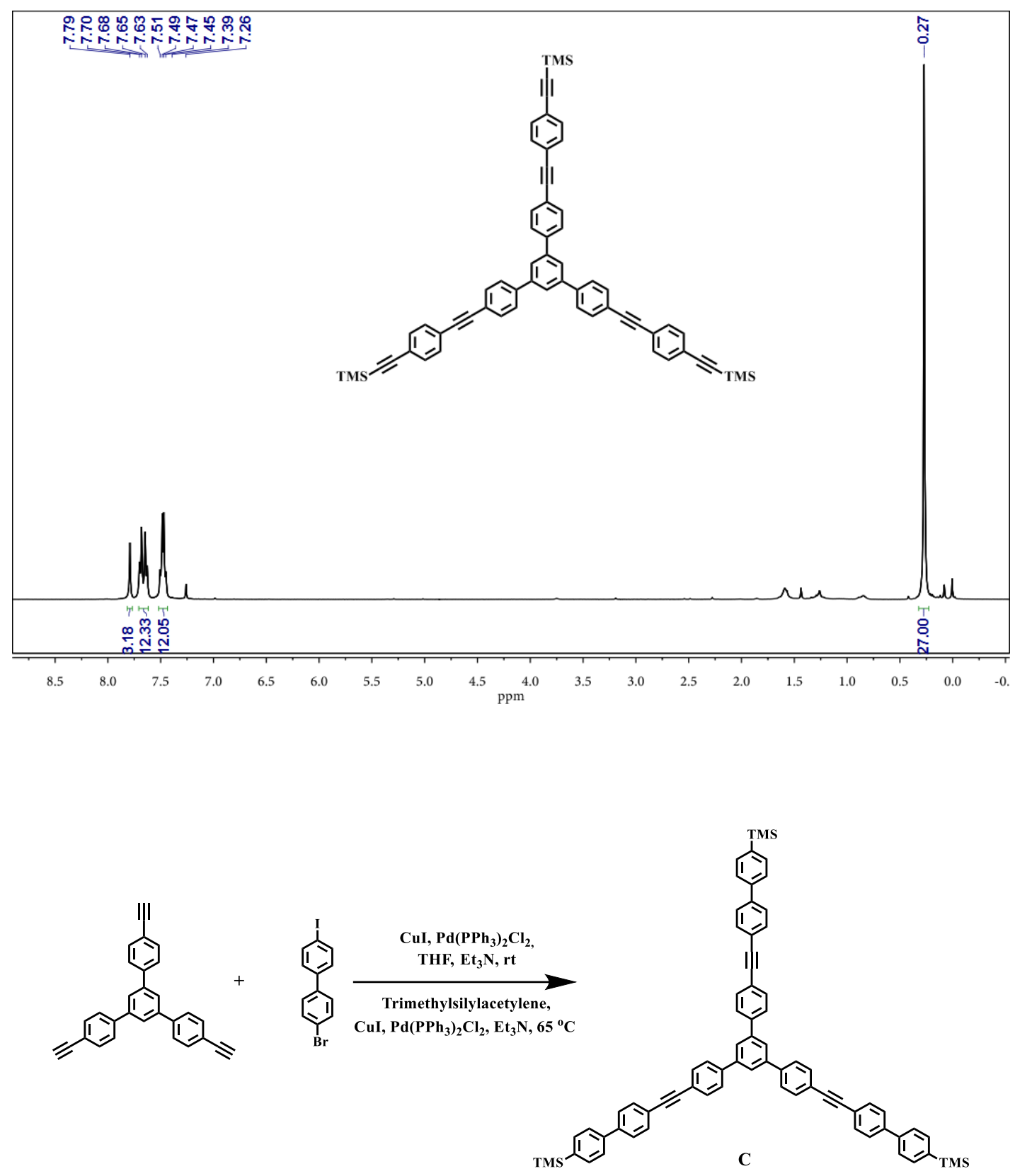

Compound C. A mixture of 1,3,5-tris[4'-(ethynyl)pheny-1)-benzene (2 g, $5.3 \mathrm{mmol}$ ), $\mathrm{Pd}\left(\mathrm{PPh}_{3}\right)_{2} \mathrm{Cl}_{2}(185 \mathrm{mg}, 0.26 \mathrm{mmol}), \mathrm{CuI}(80 \mathrm{mg}, 0.42 \mathrm{mmol})$ and 4-bromo-4'iodobiphenyl (8.6 g, $23.85 \mathrm{mmol})$ in $\mathrm{Et}_{3} \mathrm{~N} / \mathrm{THF}(1 / 2 \mathrm{v} / \mathrm{v}, 60 \mathrm{~mL})$ was purged with $\mathrm{N}_{2}$ for $10 \mathrm{~min}$. The mixture was then stirred at room temperature for $24 \mathrm{~h}$ under $\mathrm{N}_{2}$ atmosphere. The mixture was filtered through suction funnel, and the solid was washed with water, $\mathrm{MeOH}$ and diethyl ether, and dried at $60{ }^{\circ} \mathrm{C}$ under vacuum overnight to afford the intermediate product. The intermediate product $(1.5 \mathrm{~g}, 0.14 \mathrm{mmol})$, 
$\mathrm{Pd}\left(\mathrm{PPh}_{3}\right)_{2} \mathrm{Cl}_{2}(64.7 \mathrm{mg}, 0.092 \mathrm{mmol})$ and $\mathrm{CuI}(8.76 \mathrm{mg}, 0.046 \mathrm{mmol})$ were dissolved in $\mathrm{Et}_{3} \mathrm{~N}(30 \mathrm{~mL})$. The mixture was purged with $\mathrm{N}_{2}$ for $10 \mathrm{~min}$, and then trimethylsilylacetylene $(1.26 \mathrm{~g}, 12.9 \mathrm{mmol})$ was added. The suspension was stirred under $\mathrm{N}_{2}$ at $65^{\circ} \mathrm{C}$ for $24 \mathrm{~h}$. After cooling to room temperature, the mixture was extracted with dichloromethane (DCM). The organic phase was washed with brine, dried over anhydrous $\mathrm{Na}_{2} \mathrm{SO}_{4}$, and then concentrated under reduced pressure. The crude product was purified by column chromatography on silica gel (petroleum ether/EtOAc, 10:1, v/v) to afford $\mathbf{C}$ as a yellow solid $(0.92 \mathrm{~g}, 63 \%)$. ${ }^{1} \mathrm{H}$ NMR (400 $\left.\mathrm{MHz}, \mathrm{CDCl}_{3}\right) \delta 7.82$ (s, 1H), 7.70-7.53 (m, 36H), $0.28(\mathrm{~s}, 27 \mathrm{H})$.

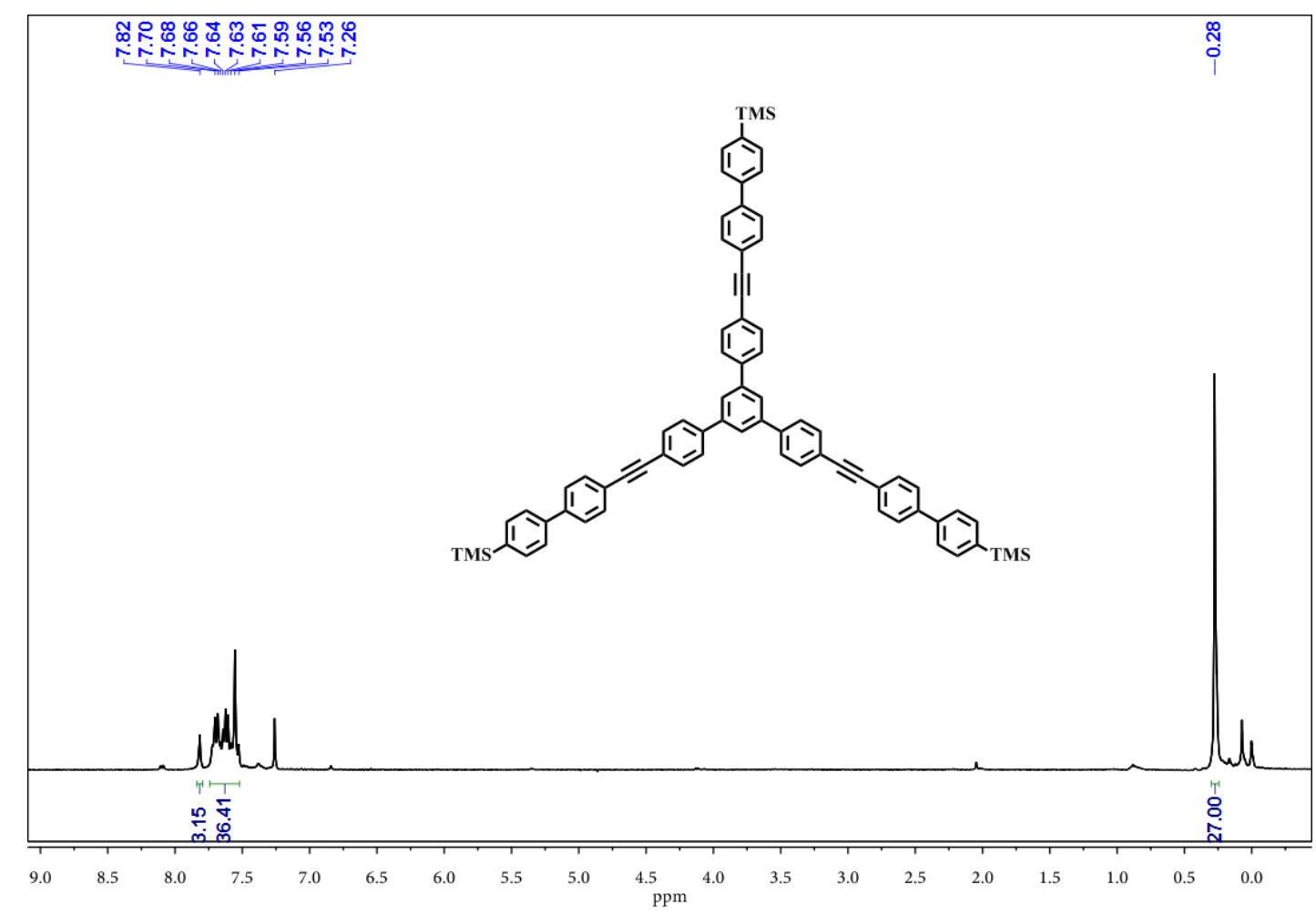

3. General procedure for photocatalysis

\subsection{The hydroxylation of arylboronic acids to phenols}
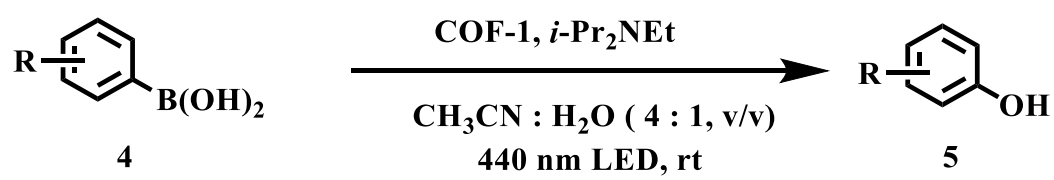

Arylboronic acids (4) $(0.5 \mathrm{mmol}), i$ - $\operatorname{Pr}_{2} \mathrm{NEt}(1.5 \mathrm{mmol})$ and COF-1 $(0.025 \mathrm{mmol})$ were dispersed in the mixture of $\mathrm{H}_{2} \mathrm{O}(1 \mathrm{~mL})$ and $\mathrm{CH}_{3} \mathrm{CN}(4 \mathrm{~mL})$. The mixture was stirred under air atmosphere at rt. After irradiation with $440 \mathrm{~nm}$ LED for $36 \mathrm{~h}$, the 
mixture was extracted with DCM $(3 \times 30 \mathrm{~mL})$. The combined organic layer was washed with brine, dried over $\mathrm{Na}_{2} \mathrm{SO}_{4}$, and then concentrated under reduced pressure. The crude product was purified by a flash chromatography on silica gel $\left(\mathrm{SiO}_{2} ; 0 \%\right.$ to $50 \%$ $\mathrm{CH}_{2} \mathrm{Cl}_{2}$ in Petroleum ether) to afford the title compound. The yields of obtained compounds was determined by ${ }^{1} \mathrm{H}$ NMR spectra using 4-bromo-1,2-dimethoxybenzene as an internal standard.

\subsection{Defluoroalkylation of 1,3 -bis(trifluoromethyl)benzene with unactivated} alkenes

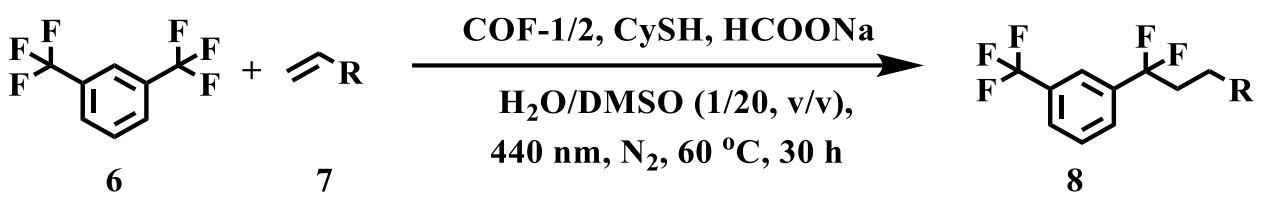

1,3-bis(trifluoromethyl)benzene (6) (0.5 mmol), alkene derivatives (7) (1.5 mmol), COF-1/2 (0.025 mmol), HCOONa $(1.5 \mathrm{mmol})$ and CySH $(0.05 \mathrm{mmol})$ were dissolved in $\mathrm{H}_{2} \mathrm{O} / \mathrm{DMSO}(1 / 20$, v/v, $5 \mathrm{~mL})$. The reaction mixture was purged with $\mathrm{N}_{2}$ for $10 \mathrm{~min}$, and then was stirred at $60{ }^{\circ} \mathrm{C}$ under irradiation with $440 \mathrm{~nm}$ LED. After $36 \mathrm{~h}$, the mixture was diluted with water and was extracted with DCM $(3 \times 30 \mathrm{~mL})$. The organic layer was washed with brine, dried over $\mathrm{Na}_{2} \mathrm{SO}_{4}$, and then concentrated under reduced pressure. The crude product was purified by column flash chromatography on silica gel ( $\mathrm{SiO}_{2} ; 0 \%$ to $40 \%$ ethyl acetate in Petroleum ether) to afford the title compound. The yields of obtained compounds was isolated yields

\subsection{Recycle experiments}

\subsubsection{Photocatalytic hydroxylation of arylboronic acids}

$4 \mathbf{a}(0.5 \mathrm{mmol}), i$-Pr $2 \mathrm{NEt}(1.5 \mathrm{mmol})$ and COF-1 $(0.025 \mathrm{mmol})$ were dispersed in the mixture of $\mathrm{H}_{2} \mathrm{O}(1 \mathrm{~mL})$ and $\mathrm{CH}_{3} \mathrm{CN}(4 \mathrm{~mL})$. The mixture was stirred at room temperature under irradiation with $440 \mathrm{~nm}$ LED for $30 \mathrm{~h}$. The COF catalyst was separated by centrifugation, washed with fresh THF for three times and then dried at $60{ }^{\circ} \mathrm{C}$ under vacuum for $1 \mathrm{~h}$. The recovered solid could be used for the next runs directly.

\subsubsection{The defluoroalkylation of 1,3-bis(trifluorometh- yl)benzene}

1,3-bis(trifluoromethyl)benzene (6) $(0.5 \mathrm{mmol})$, alkene derivatives (7a) $(1.5 \mathrm{mmol})$, COF-1 (0.025 mmol), HCOONa (1.5 mmol) and CySH $(0.05 \mathrm{mmol})$ were dissolved in $\mathrm{H}_{2} \mathrm{O} / \mathrm{DMSO}(1 / 20$, v/v, $5 \mathrm{~mL})$. The reaction mixture was purged with $\mathrm{N}_{2}$ for $10 \mathrm{~min}$, 
and then was stirred at $60{ }^{\circ} \mathrm{C}$ under irradiation with $440 \mathrm{~nm}$ LED for $36 \mathrm{~h}$. The COF catalyst was separated by centrifugation, washed with fresh methanol and THF for three times and then dried at $60{ }^{\circ} \mathrm{C}$ under vacuum for $1 \mathrm{~h}$. The recovered solid could be used for the next runs directly.

\section{Figure S1. FT-IR spectra}
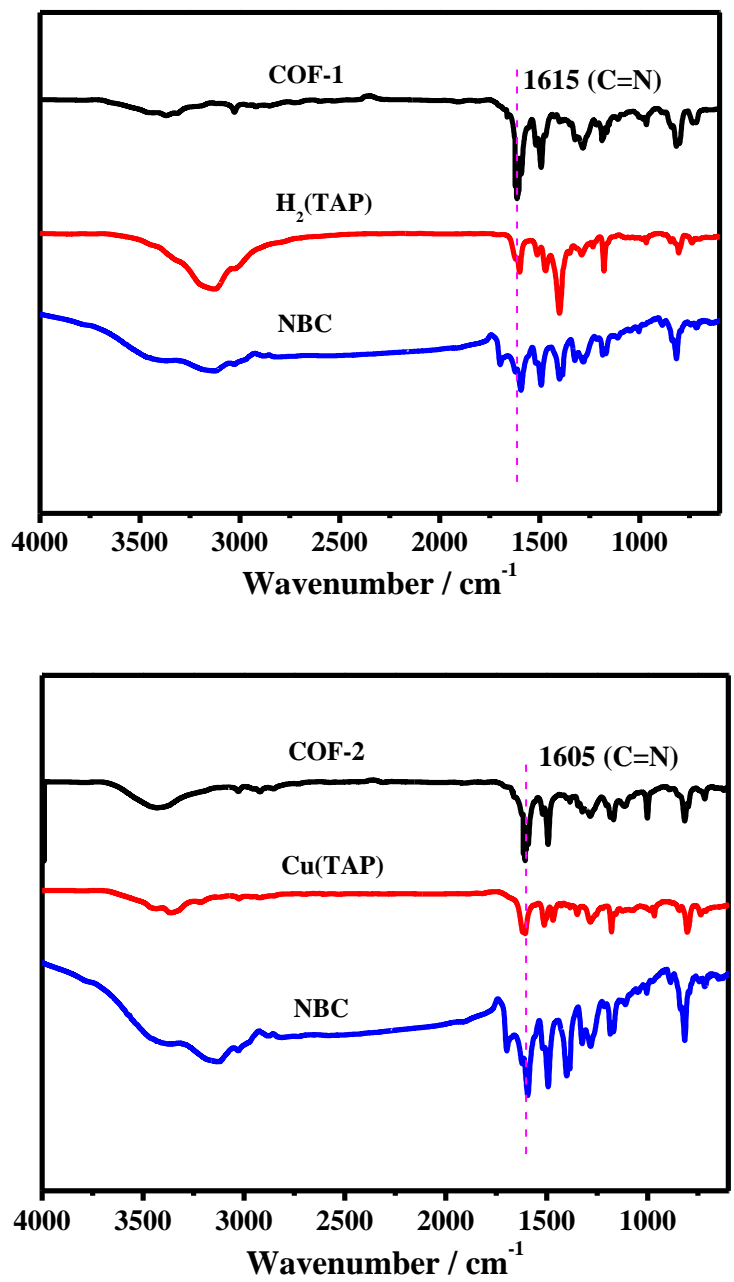


\section{Figure S2. Solid-state ${ }^{13}$ C NMR spectra}
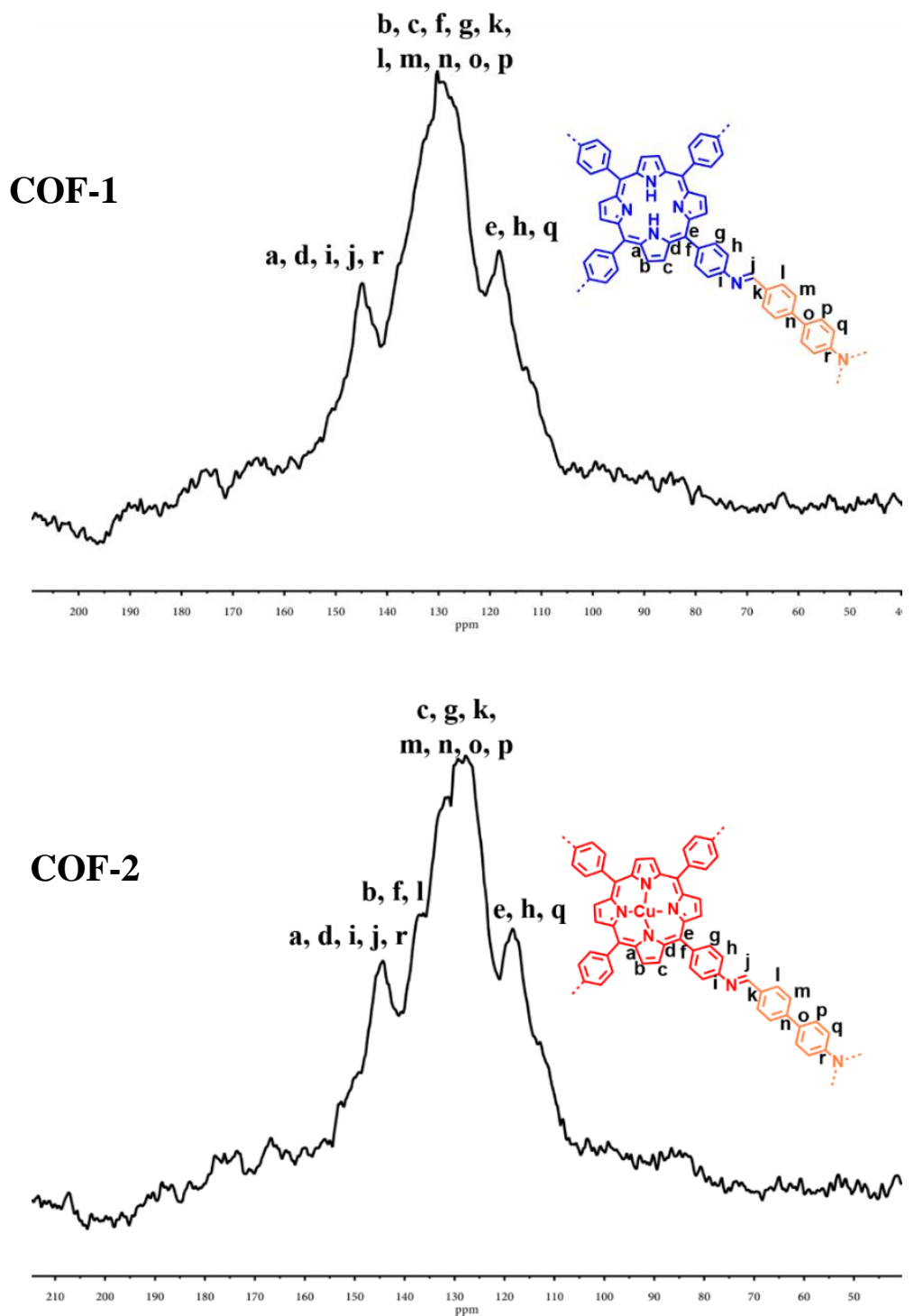
6. Figure S3. TGA curves

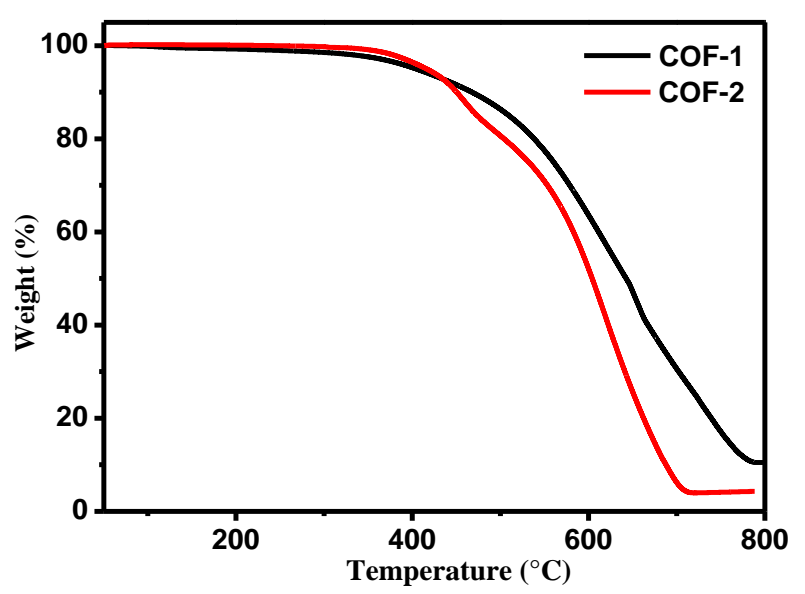

\section{Figure S4. SEM images}

a)

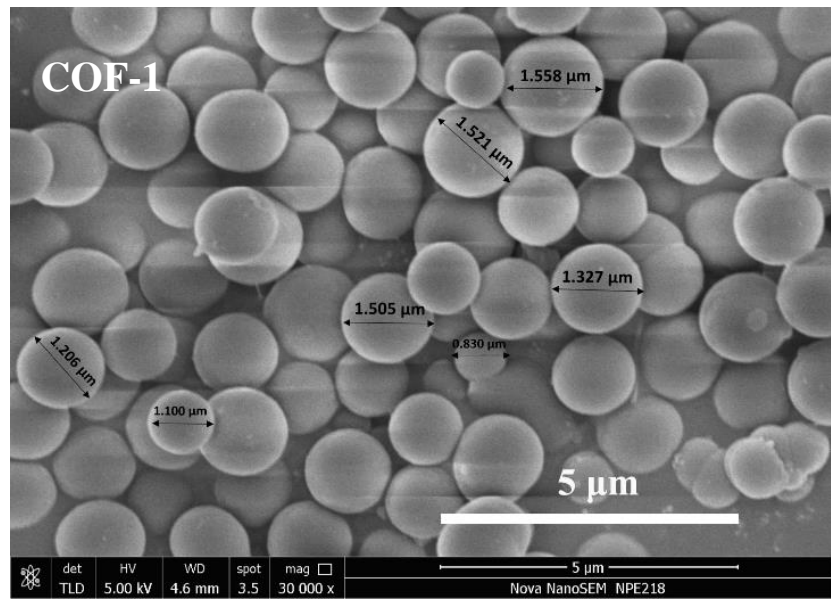

b)

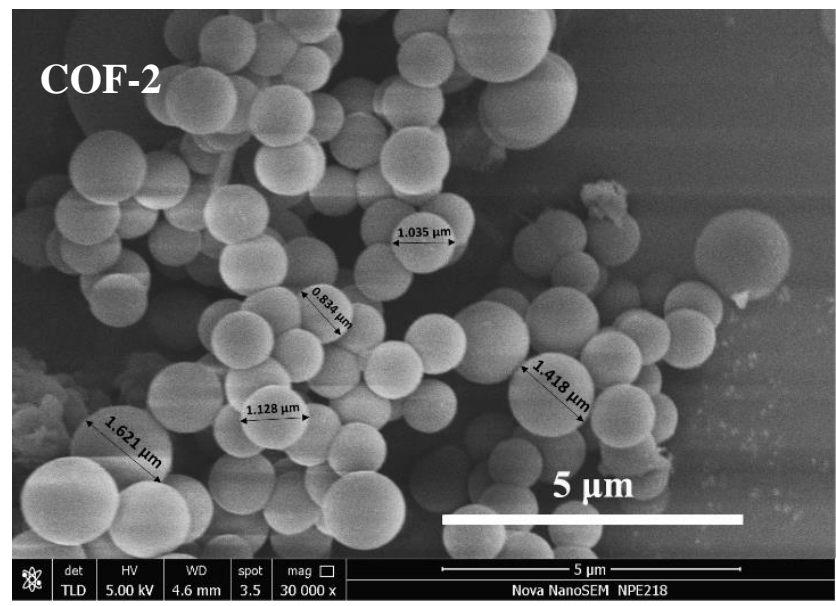




\section{Figures S5-S23. Structural modeling and PXRD analysis of the COFs}

\subsection{Structure Simulation}

Molecular modeling of COFs was generated with the Materials Studio (version 7.0) suite of programs. Pawley refinement was carried out using Reflex, a software package for crystal determination from PXRD pattern. Unit cell dimension was set to the theoretical parameters. The Pawley refinement was performed to optimize the lattice parameters iteratively until the $R_{\mathrm{wp}}$ value converges and the overlay of the observed with refined profiles shows good agreement. The lattice models (e.g., cell parameters, atomic positions, and total energies) were then fully optimized using MS Forcite molecular dynamics module (universal force fields, Ewald summations) method.

For COF-1, considering the geometry of the precursors and the connection patterns, only a few topologies (eg. tbo, pto, ffc, fjh, etc.) are reasonable according to RCSR. The lattice models (e.g., cell parameters, atomic positions, and total energies) were then fully optimized.

Figure S5. Space-filling models of COF-1 with different non-interpenetrated nets: a) pto, b) ffc, c) tbo, d) fjh. (Carbon, gray; Nitrogen, blue; Hydrogen, white)
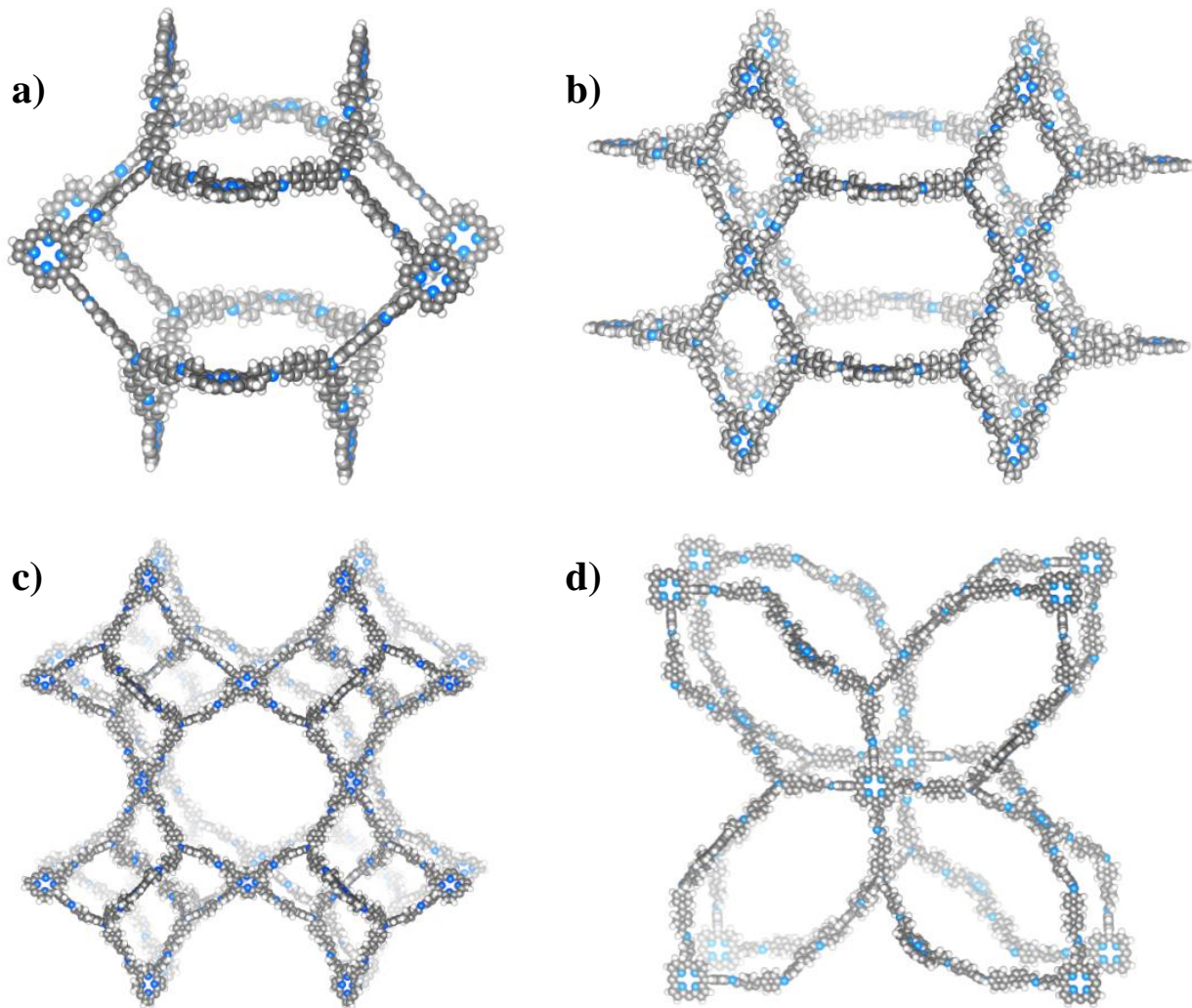

d)

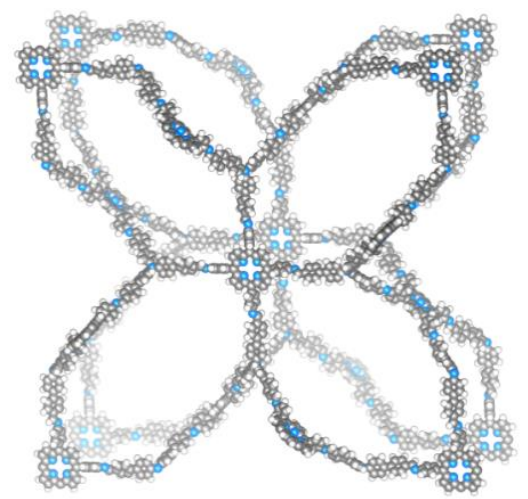


Figure S6. PXRD patterns of COF-1 after Pawley refinement: PXRD profiles of experimental pattern (black curve), Pawley refined (red curve), and calculated (blue curve) patterns from the modeled structure with the non-interpenetrated pto topology; their difference (green curve).

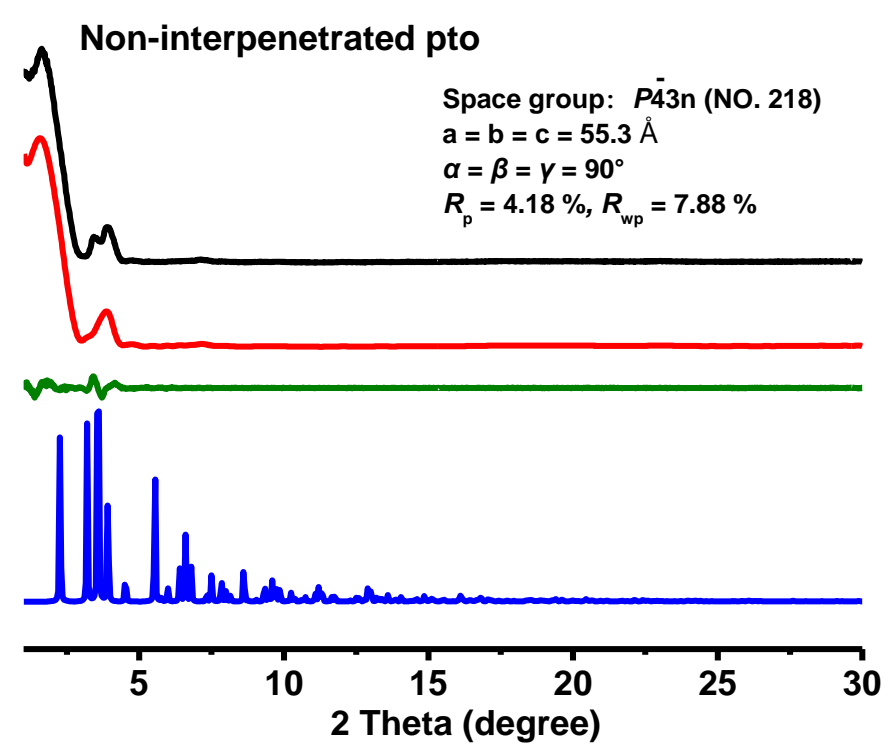

Figure S7. PXRD patterns of COF-1 after Pawley refinement: PXRD profiles of experimental pattern (black curve), Pawley refined (red curve), and calculated (blue curve) patterns from the modeled structure with the non-interpenetrated ffc topology; their difference (green curve).

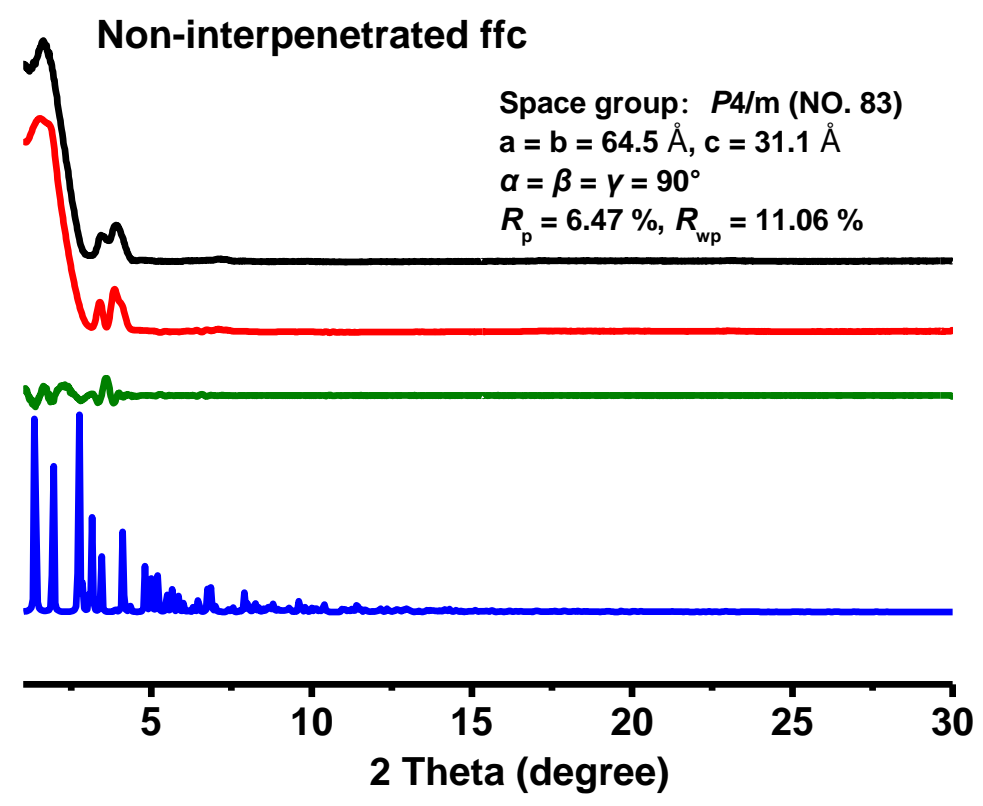


Figure S8. PXRD patterns of COF-1 after Pawley refinement: PXRD profiles of experimental pattern (black curve), Pawley refined (red curve), and calculated (blue curve) patterns from the modeled structure with the non-interpenetrated fjh topology; their difference (green curve).

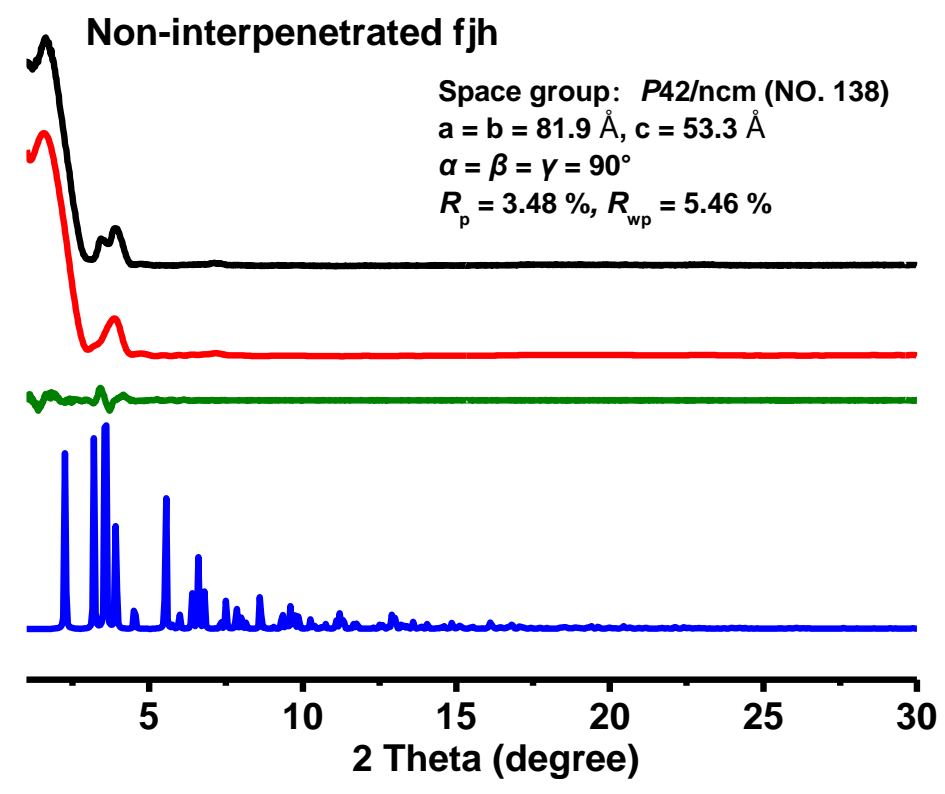

Figure S9. Space-filling models of COF-1 with different interpenetrated nets: a) pto, b) ffc, c) tbo, d) fjh.

a)

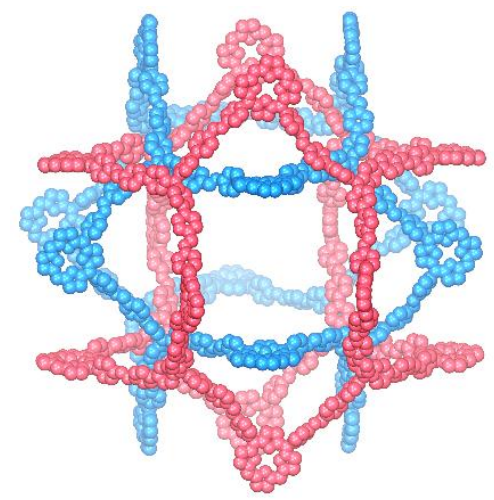

c)

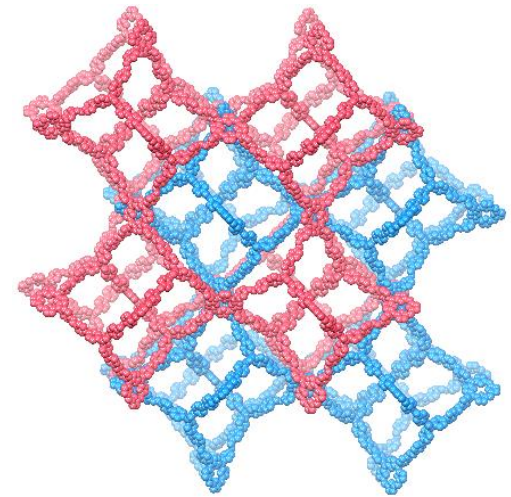

b)

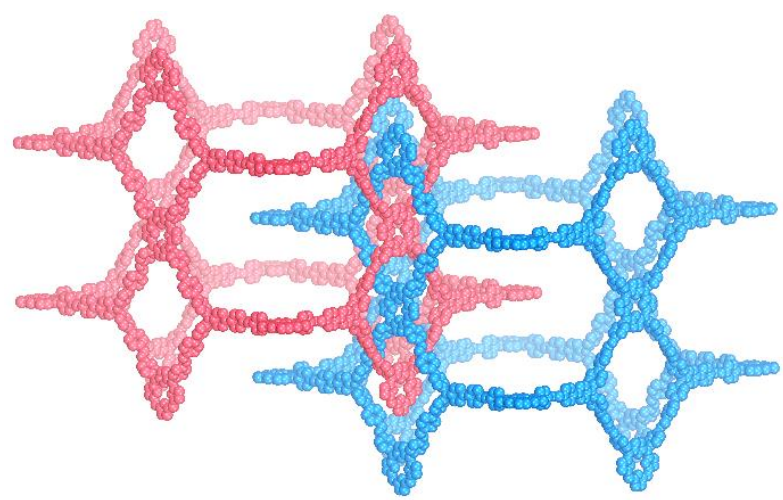

d)

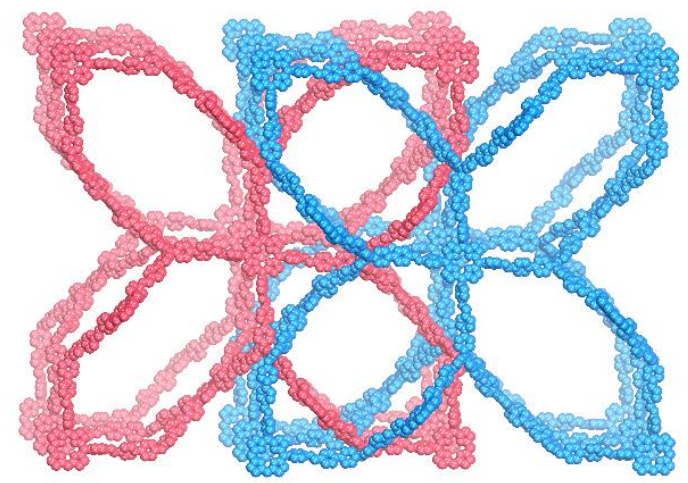


Figure S10. PXRD patterns of COF-1 after Pawley refinement: PXRD profiles of experimental pattern (black curve), Pawley refined (red curve), and calculated (blue curve) patterns from the modeled structure with the interpenetrated pto topology; their difference (green curve).

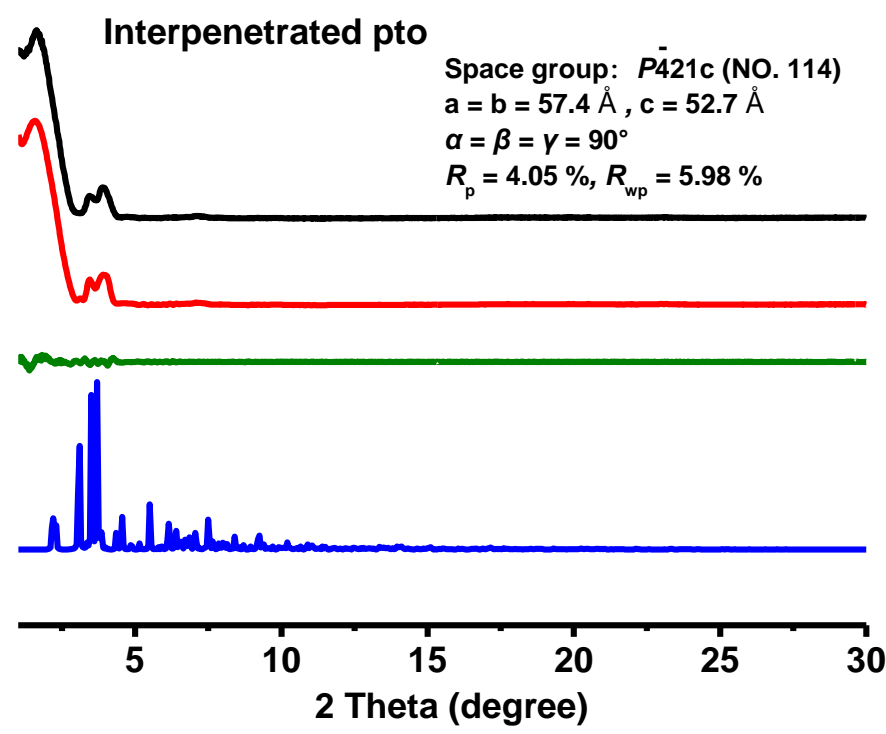

Figure S11. PXRD patterns of COF-1 after Pawley refinement: PXRD profiles of experimental pattern (black curve), Pawley refined (red curve), and calculated (blue curve) patterns from the modeled structure with the interpenetrated ffc topology; their difference (green curve).

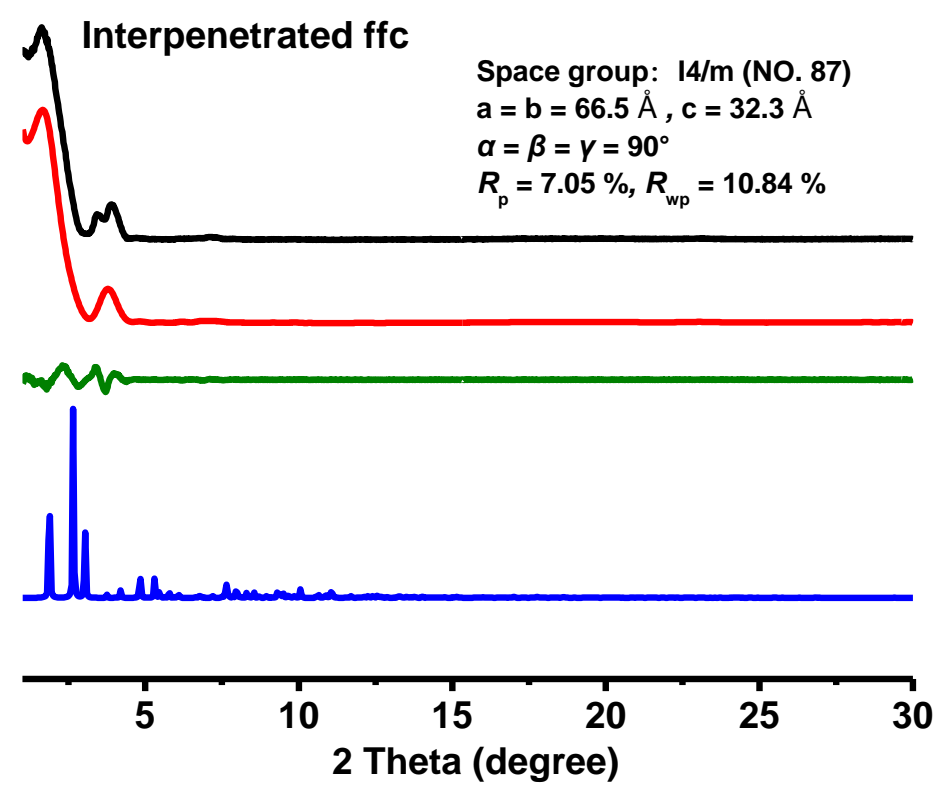


Figure S12. PXRD patterns of COF-1 after Pawley refinement: PXRD profiles of experimental pattern (black curve), Pawley refined (red curve), and calculated (blue curve) patterns from the modeled structure with the interpenetrated tbo topology; their difference (green curve).

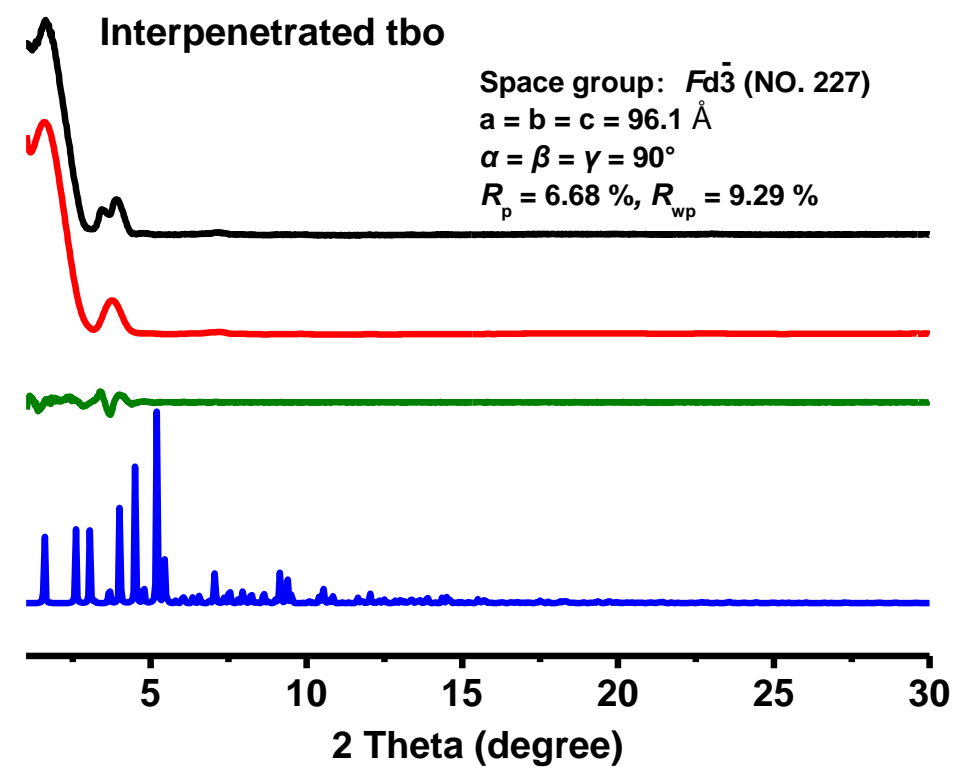

Figure S13. PXRD patterns of COF-1 after Pawley refinement: PXRD profiles of experimental pattern (black curve), Pawley refined (red curve), and calculated (blue curve) patterns from the modeled structure with the interpenetrated fjh topology; their difference (green curve).

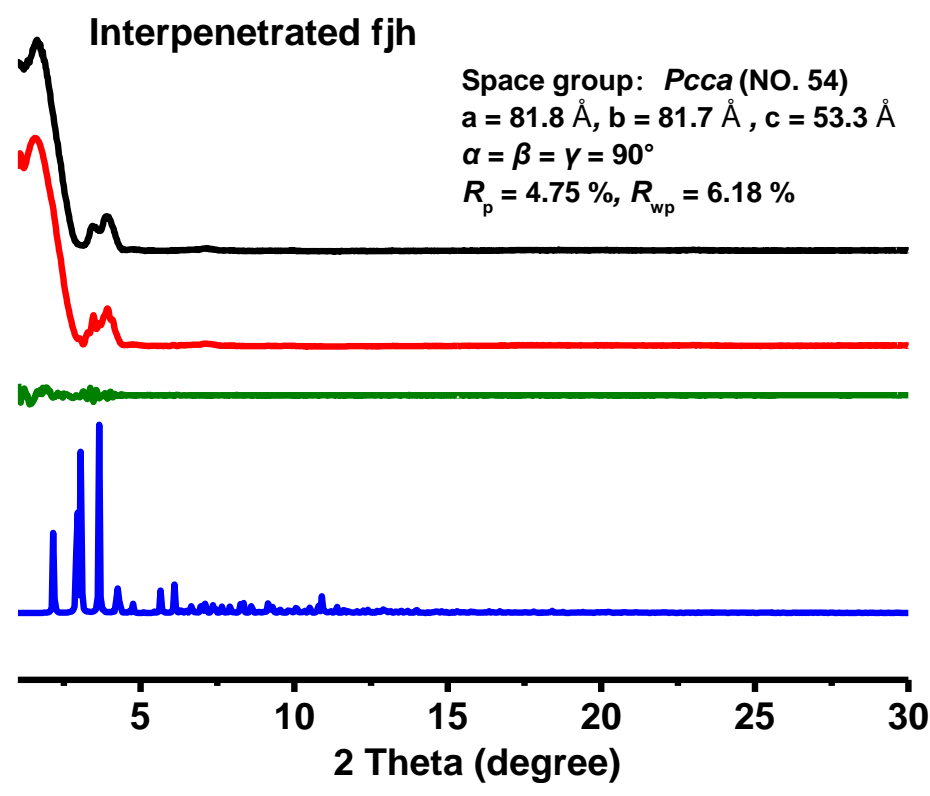


Figure S14. Space-filling models of COF-2 with different nets: a) pto, b) ffc, c) tbo, d) fjh. (Carbon, gray; Nitrogen, blue; Hydrogen, white.)

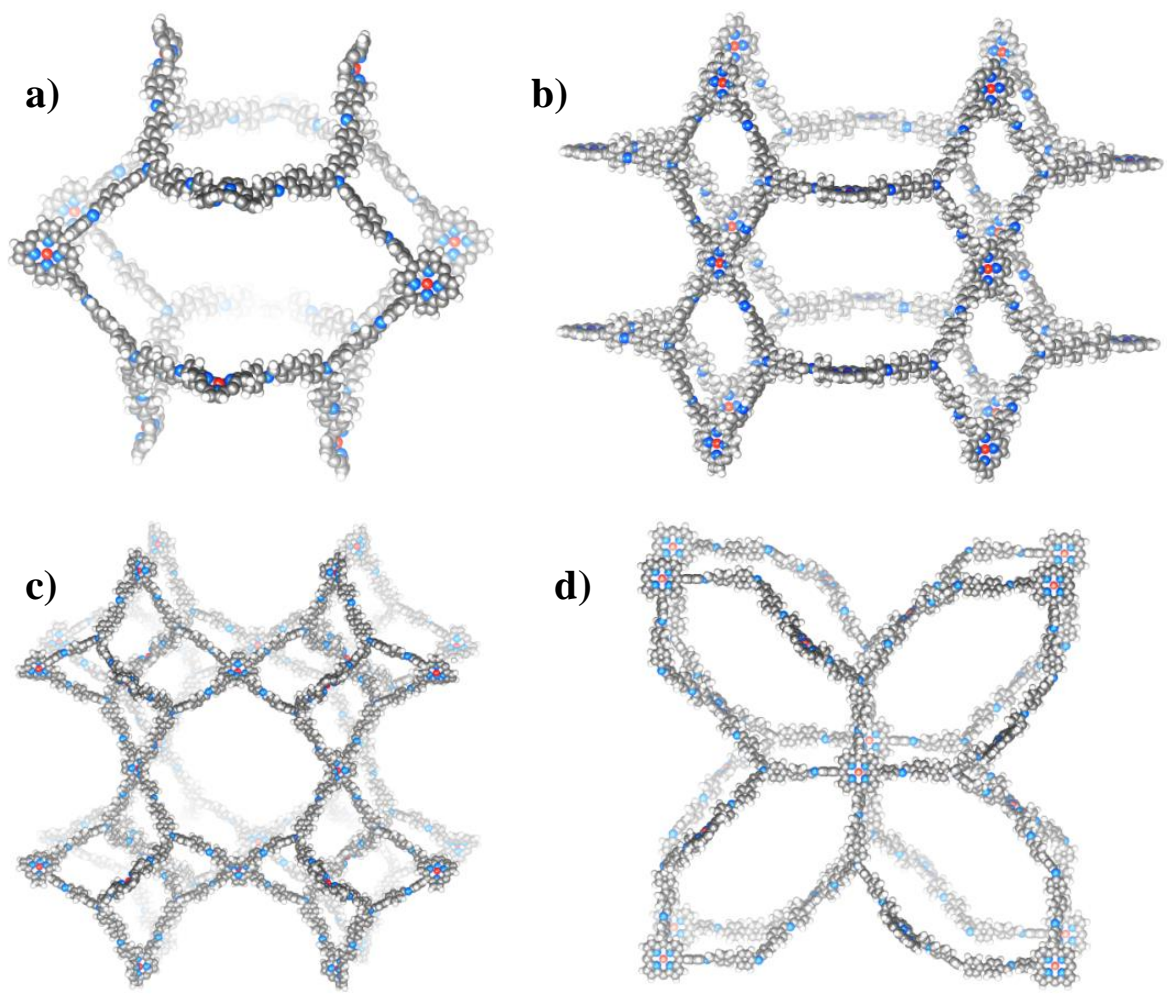

Figure S15. PXRD patterns of COF-2 after Pawley refinement: PXRD profiles of experimental pattern (black curve), Pawley refined (red curve), and calculated (blue curve) patterns from the modeled structure with the non-interpenetrated pto topology; their difference (green curve).

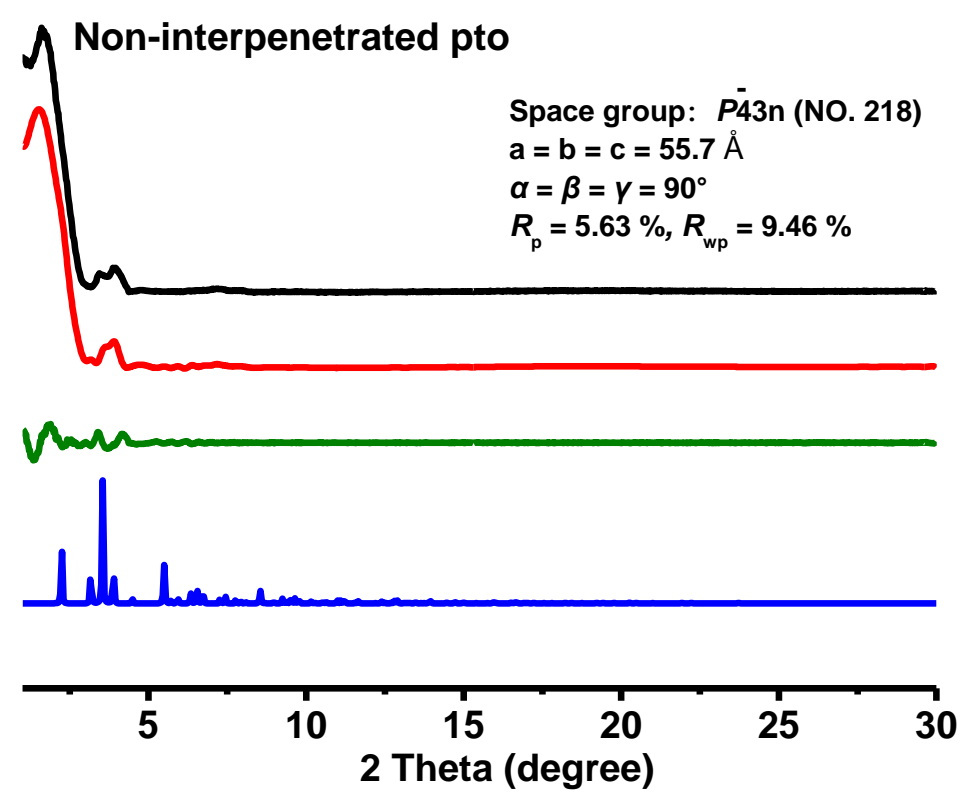


Figure S16. PXRD patterns of COF-2 after Pawley refinement: PXRD profiles of experimental pattern (black curve), Pawley refined (red curve), and calculated (blue curve) patterns from the modeled structure with the non-interpenetrated ffc topology; their difference (green curve).

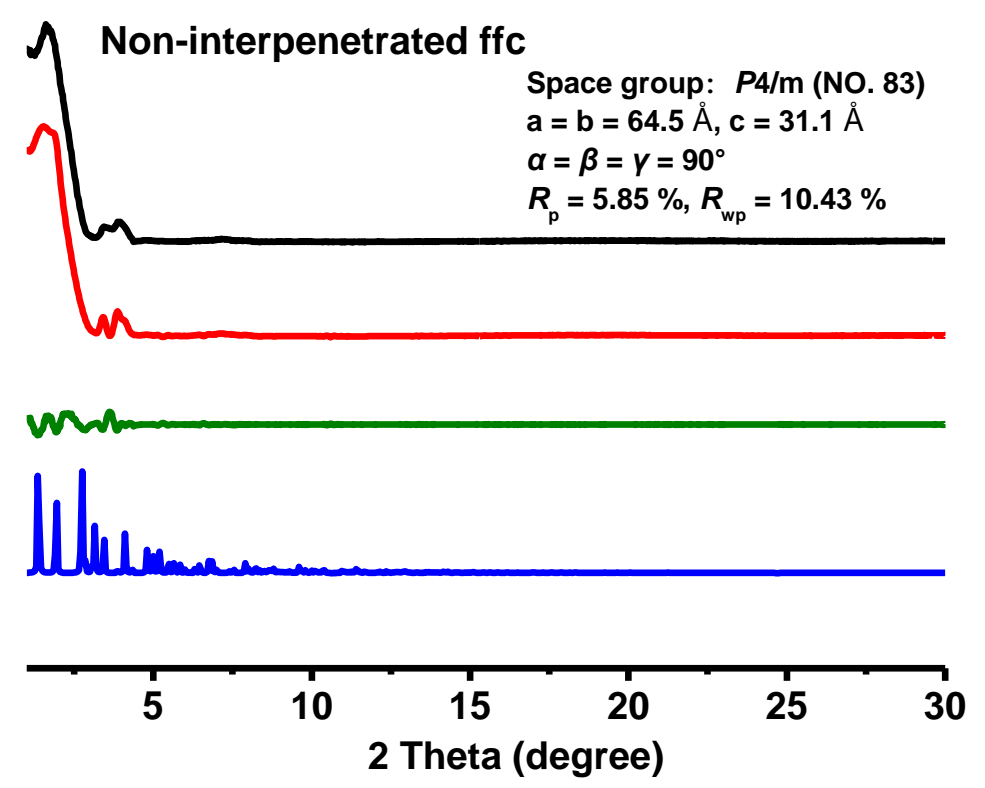

Figure S17. PXRD patterns of COF-2 after Pawley refinement: PXRD profiles of experimental pattern (black curve), Pawley refined (red curve), and calculated (blue curve) patterns from the modeled structure with the non-interpenetrated fjh topology; their difference (green curve).

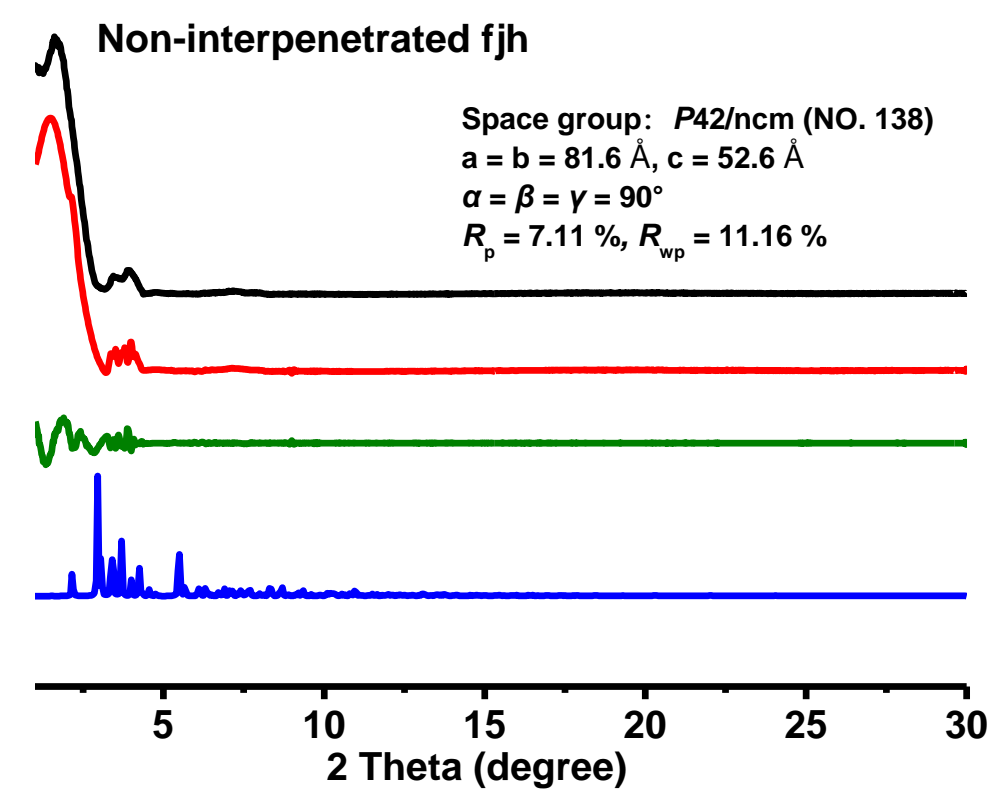


Figure S18. Space-filling models of COF-2 with different interpenetrated nets: a) pto, b) ffc, c) tbo, d) fjh.
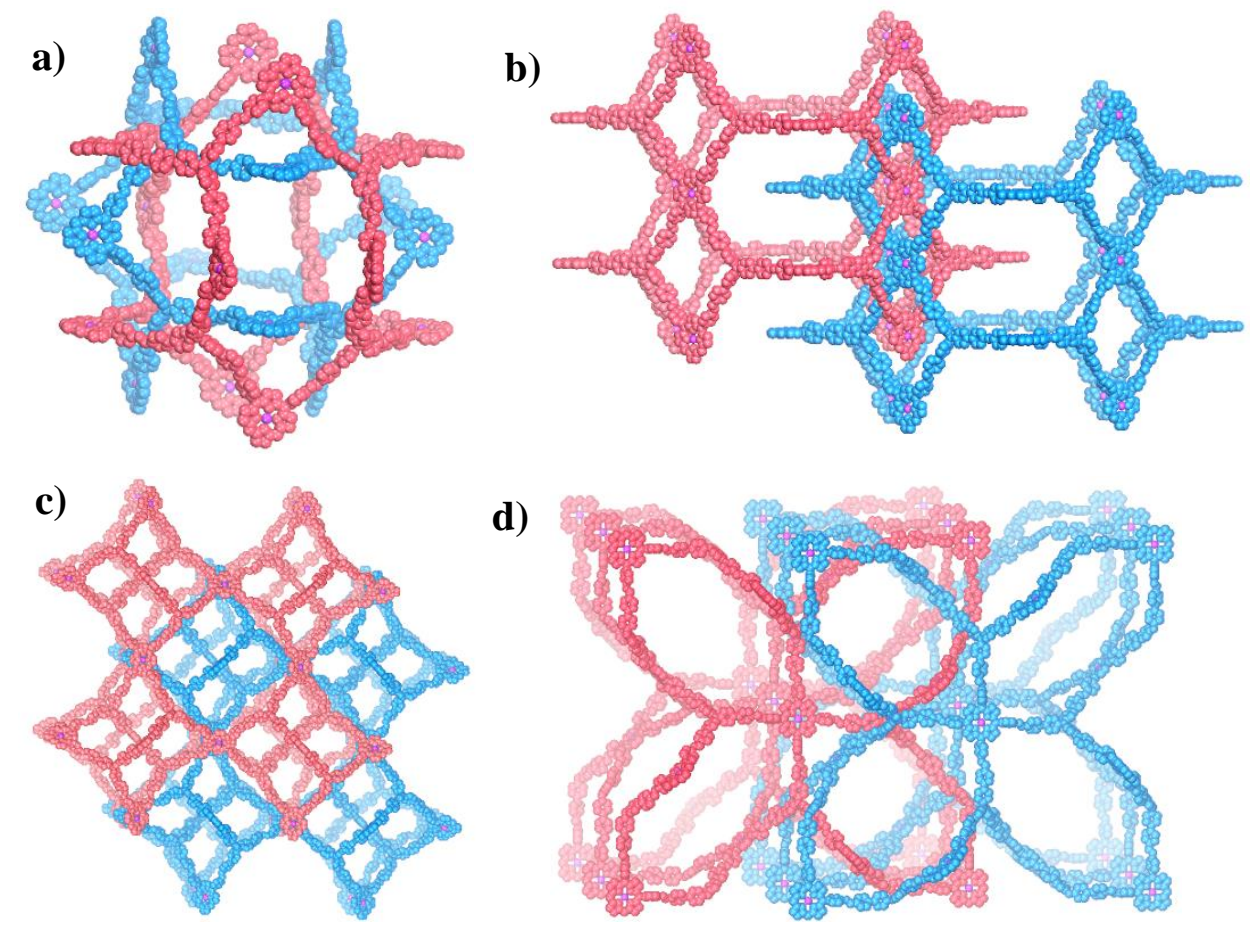

d)

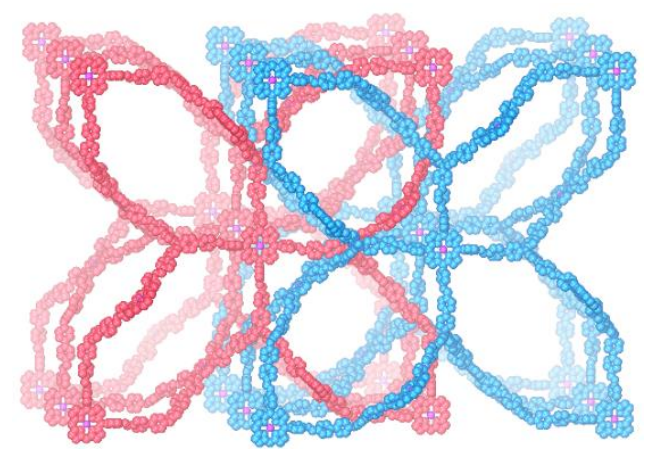

Figure S19. PXRD patterns of COF-2 after Pawley refinement: PXRD profiles of experimental pattern (black curve), Pawley refined (red curve), and calculated (blue curve) patterns from the modeled structure with the interpenetrated pto topology; their difference (green curve).

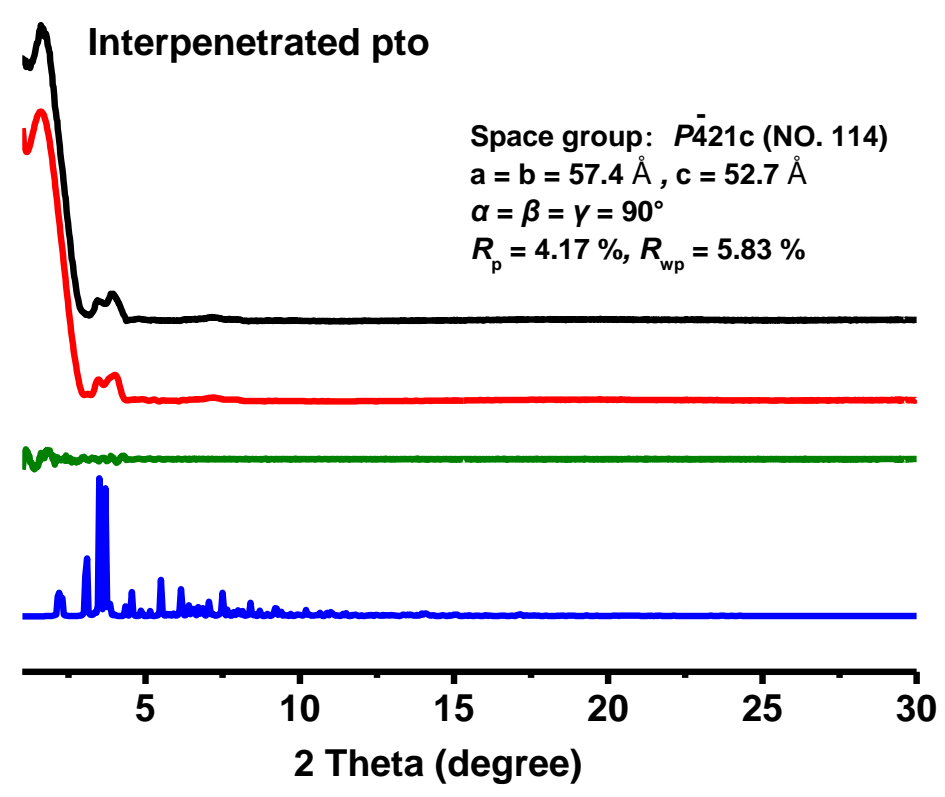


Figure S20. PXRD patterns of COF-2 after Pawley refinement: PXRD profiles of experimental pattern (black curve), Pawley refined (red curve), and calculated (blue curve) patterns from the modeled structure with the interpenetrated ffc topology; their difference (green curve).

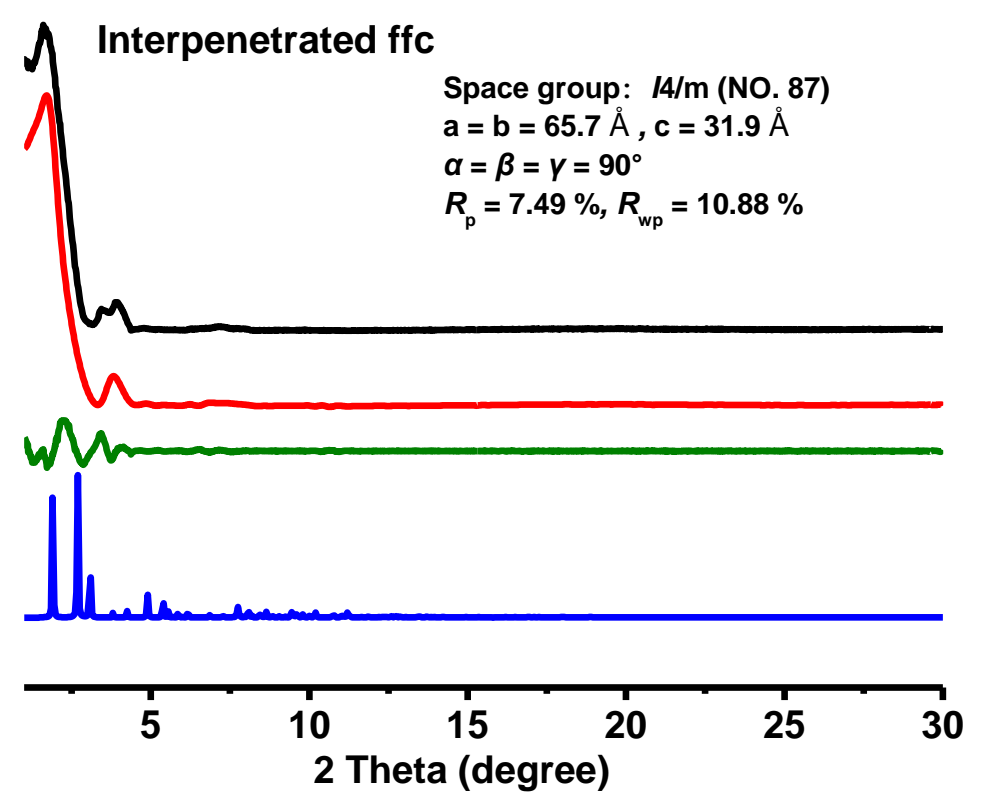

Figure S21. PXRD patterns of COF-2 after Pawley refinement: PXRD profiles of experimental pattern (black curve), Pawley refined (red curve), and calculated (blue curve) patterns from the modeled structure with the interpenetrated tbo topology; their difference (green curve).

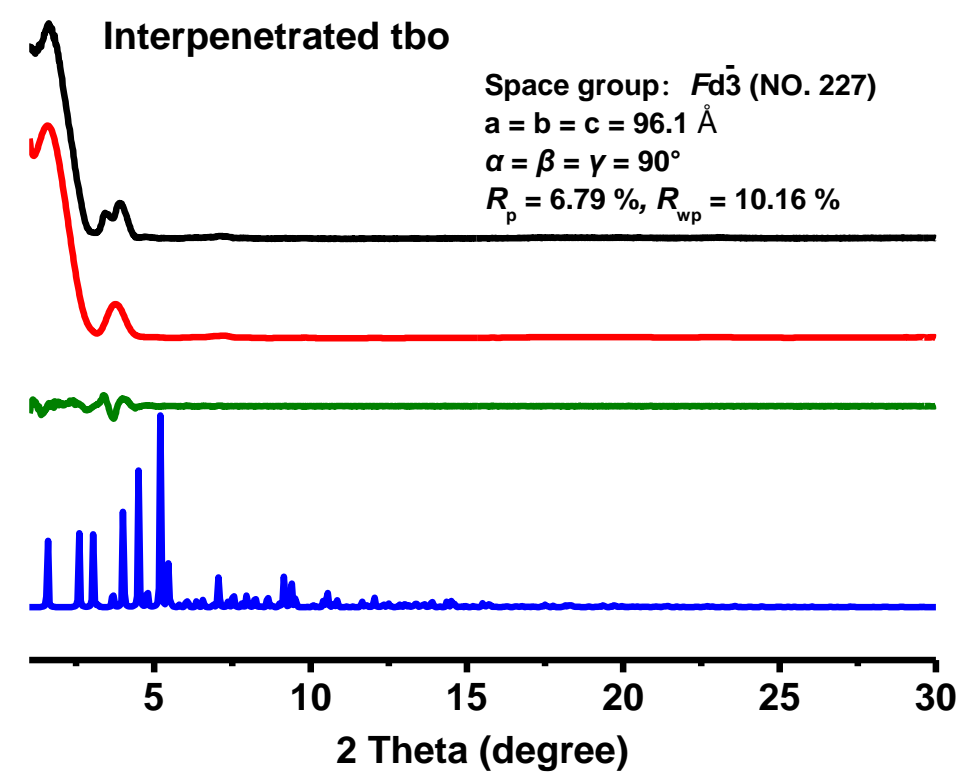


Figure S22. PXRD patterns of COF-2 after Pawley refinement: PXRD profiles of experimental pattern (black curve), Pawley refined (red curve), and calculated (blue curve) patterns from the modeled structure with the interpenetrated fjh topology; their difference (green curve).

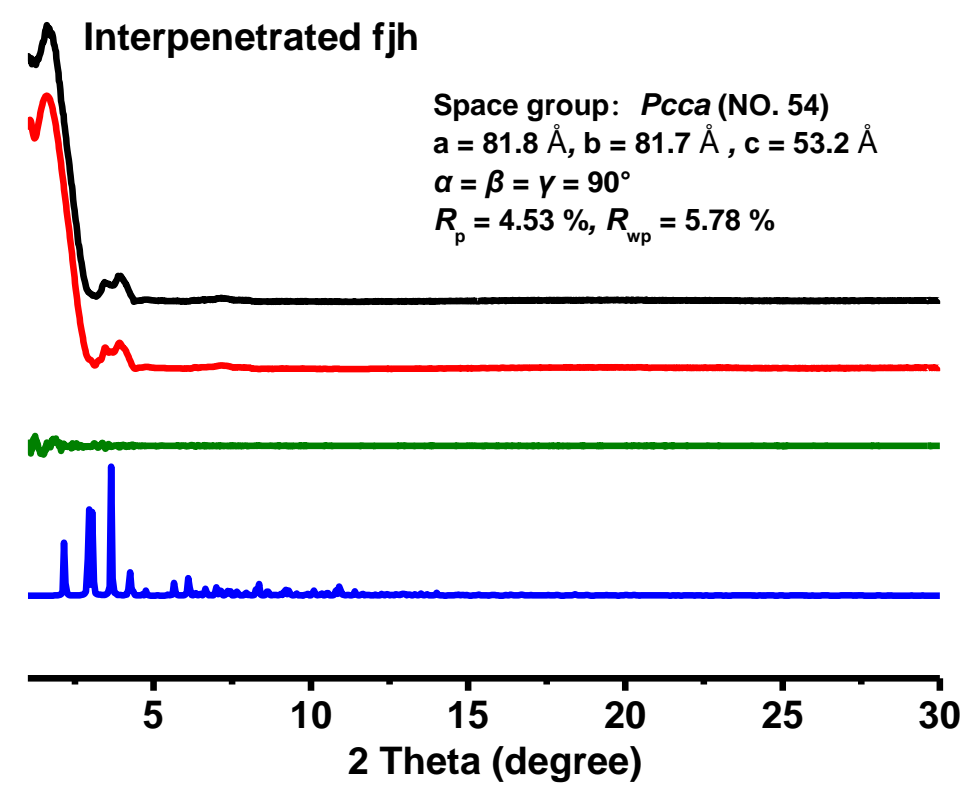

Figure S23. The structures of COF-2: a) representative section of the crystal structure of COF-2 showing the polyhedral cages. b) the structure of COF-2 with tbo topology. c) the nanochannels of COF-2.

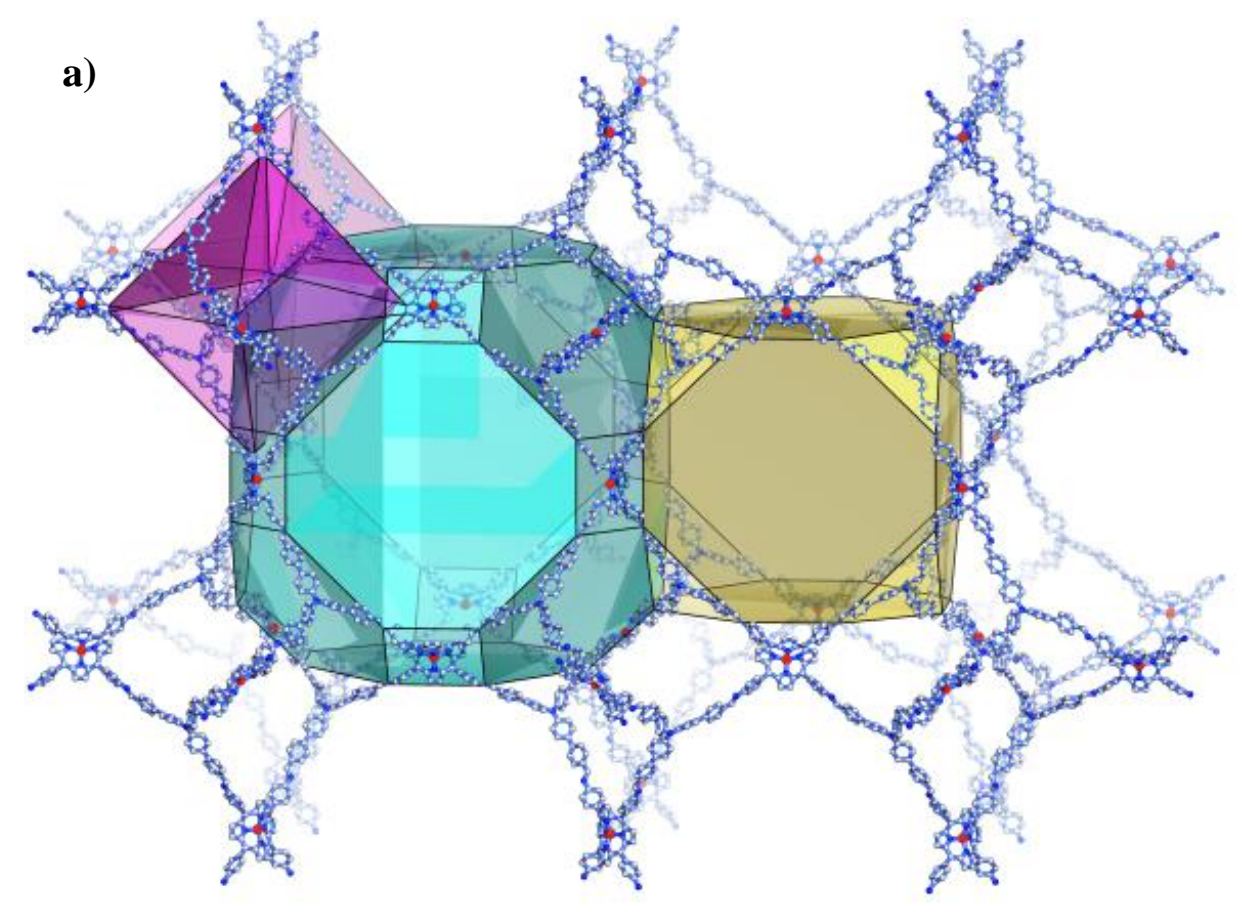


b)

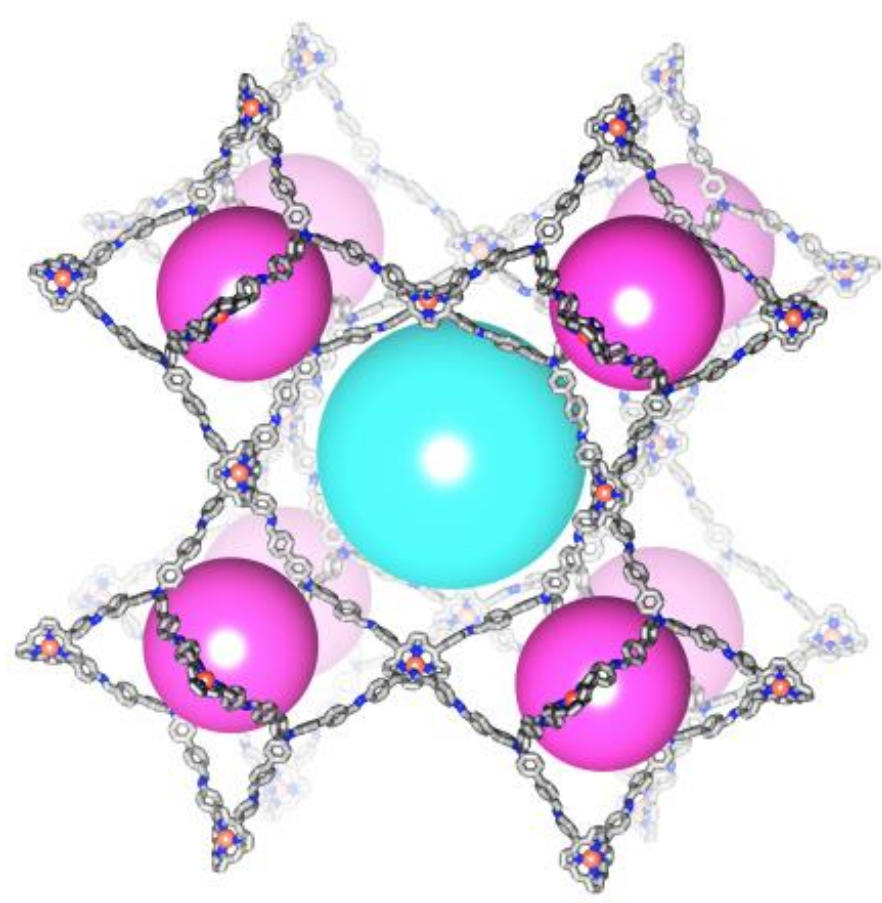

c)

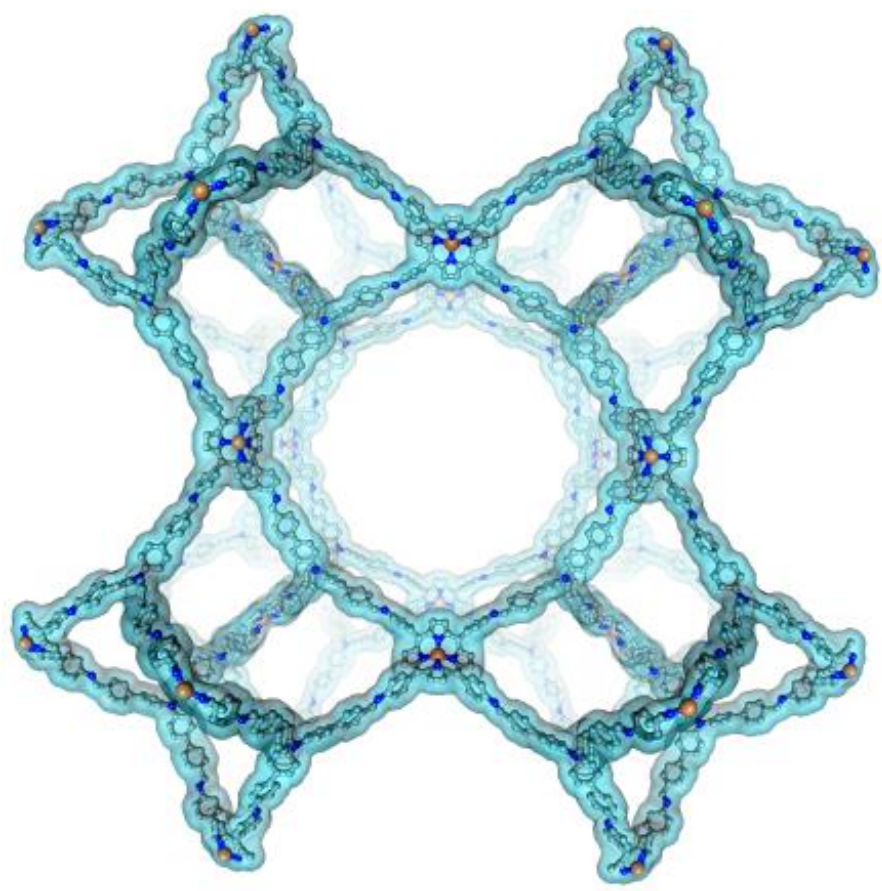

\section{Tables S1-S16. Fractional atomic coordinates and unit cell parameters}

Table S1. Fractional atomic coordinates for the unit cell of COF-1 with the noninterpenetrated pto topology.

\begin{tabular}{|c|c|c|c|}
\hline \\
\hline \multicolumn{4}{|c|}{$\mathrm{a}=\mathrm{b}=\mathrm{c}=55.2619 \AA, \alpha=\beta=\gamma=90^{\circ}$} \\
\hline Atom & $\mathrm{X}$ & $\mathrm{y}$ & $\mathrm{Z}$ \\
\hline
\end{tabular}




\begin{tabular}{|c|c|c|c|}
\hline $\mathrm{C}$ & -0.01425 & 0.55186 & 0.24778 \\
\hline $\mathrm{N}$ & 0.0034 & 0.53563 & 0.25 \\
\hline $\mathrm{C}$ & 0.02387 & 0.54822 & 0.24852 \\
\hline $\mathrm{C}$ & 0.01915 & 0.57215 & 0.24518 \\
\hline $\mathrm{C}$ & -0.00497 & 0.57445 & 0.24466 \\
\hline $\mathrm{C}$ & 0.04695 & 0.53876 & 0.25075 \\
\hline $\mathrm{C}$ & 0.06724 & 0.5556 & 0.25157 \\
\hline $\mathrm{C}$ & 0.07167 & 0.56883 & 0.27219 \\
\hline $\mathrm{C}$ & 0.09063 & 0.58484 & 0.27292 \\
\hline $\mathrm{C}$ & 0.10553 & 0.58777 & 0.2532 \\
\hline $\mathrm{C}$ & 0.10093 & 0.57457 & 0.2324 \\
\hline $\mathrm{C}$ & 0.08194 & 0.55857 & 0.23168 \\
\hline $\mathrm{N}$ & 0.1245 & 0.60482 & 0.25491 \\
\hline $\mathrm{C}$ & 0.14223 & 0.60726 & 0.24032 \\
\hline $\mathrm{C}$ & 0.16021 & 0.626 & 0.24412 \\
\hline $\mathrm{C}$ & 0.17914 & 0.62829 & 0.22806 \\
\hline $\mathrm{C}$ & 0.19618 & 0.64611 & 0.23112 \\
\hline $\mathrm{C}$ & 0.19464 & 0.66199 & 0.25035 \\
\hline $\mathrm{C}$ & 0.17574 & 0.65957 & 0.2665 \\
\hline $\mathrm{C}$ & 0.15869 & 0.64178 & 0.26342 \\
\hline $\mathrm{C}$ & 0.2125 & 0.68117 & 0.25342 \\
\hline $\mathrm{C}$ & 0.22081 & 0.69403 & 0.23368 \\
\hline $\mathrm{C}$ & 0.23724 & 0.71242 & 0.23662 \\
\hline $\mathrm{C}$ & 0.24575 & 0.7183 & 0.25934 \\
\hline $\mathrm{C}$ & 0.23761 & 0.70534 & 0.27905 \\
\hline $\mathrm{C}$ & 0.22115 & 0.68698 & 0.27613 \\
\hline $\mathrm{H}$ & 0.03179 & 0.58653 & 0.24334 \\
\hline $\mathrm{H}$ & -0.01459 & 0.59095 & 0.2424 \\
\hline $\mathrm{H}$ & 0.0604 & 0.56677 & 0.28765 \\
\hline $\mathrm{H}$ & 0.09379 & 0.595 & 0.28896 \\
\hline $\mathrm{H}$ & 0.11154 & 0.5767 & 0.21655 \\
\hline $\mathrm{H}$ & 0.07856 & 0.54859 & 0.21556 \\
\hline $\mathrm{H}$ & 0.14437 & 0.59549 & 0.22528 \\
\hline $\mathrm{H}$ & 0.18073 & 0.6163 & 0.21309 \\
\hline $\mathrm{H}$ & 0.21069 & 0.64733 & 0.21854 \\
\hline $\mathrm{H}$ & 0.17389 & 0.67172 & 0.28131 \\
\hline $\mathrm{H}$ & 0.14425 & 0.6405 & 0.27608 \\
\hline $\mathrm{H}$ & 0.21425 & 0.69021 & 0.21601 \\
\hline $\mathrm{H}$ & 0.24308 & 0.72232 & 0.2212 \\
\hline $\mathrm{H}$ & 0.24411 & 0.70941 & 0.29669 \\
\hline $\mathrm{H}$ & 0.21535 & 0.67716 & 0.29163 \\
\hline $\mathrm{N}$ & 0.26242 & 0.26242 & 0.73758 \\
\hline
\end{tabular}


Table S2. Fractional atomic coordinates for the unit cell of COF-1 with the noninterpenetrated fjh topology.

\begin{tabular}{|c|c|c|c|}
\hline \multicolumn{4}{|c|}{ Space group: $P 42 / \mathrm{ncm}$} \\
\hline \multicolumn{4}{|c|}{$\mathrm{a}=\mathrm{b}=81.8708 \AA, \mathrm{c}=53.3392 \AA, \alpha=\beta=\gamma=90^{\circ}$} \\
\hline Atom & $\mathrm{x}$ & $\mathrm{y}$ & $\mathrm{Z}$ \\
\hline $\mathrm{C}$ & 0.11558 & 0.36581 & 0.44217 \\
\hline $\mathrm{C}$ & 0.13196 & 0.37982 & 0.41741 \\
\hline $\mathrm{C}$ & 0.14801 & 0.40087 & 0.51554 \\
\hline $\mathrm{C}$ & 0.15846 & 0.41326 & 0.5081 \\
\hline $\mathrm{C}$ & 0.1453 & 0.39655 & 0.44989 \\
\hline $\mathrm{C}$ & 0.15498 & 0.40509 & 0.43037 \\
\hline $\mathrm{C}$ & 0.1481 & 0.41795 & 0.41662 \\
\hline $\mathrm{C}$ & 0.15743 & 0.4262 & 0.3987 \\
\hline $\mathrm{C}$ & 0.17374 & 0.42184 & 0.3944 \\
\hline $\mathrm{C}$ & 0.18049 & 0.40867 & 0.4079 \\
\hline $\mathrm{C}$ & 0.17116 & 0.40043 & 0.42581 \\
\hline $\mathrm{N}$ & 0.18285 & 0.43091 & 0.37601 \\
\hline $\mathrm{C}$ & 0.19864 & 0.43097 & 0.3735 \\
\hline $\mathrm{C}$ & 0.20663 & 0.44084 & 0.35385 \\
\hline $\mathrm{C}$ & 0.22372 & 0.44081 & 0.35212 \\
\hline $\mathrm{C}$ & 0.23163 & 0.45015 & 0.3339 \\
\hline $\mathrm{C}$ & 0.22261 & 0.45974 & 0.31699 \\
\hline $\mathrm{C}$ & 0.20547 & 0.45969 & 0.31863 \\
\hline $\mathrm{C}$ & 0.19755 & 0.45034 & 0.3369 \\
\hline $\mathrm{C}$ & 0.2311 & 0.46977 & 0.29779 \\
\hline $\mathrm{C}$ & 0.24549 & 0.47825 & 0.30388 \\
\hline $\mathrm{C}$ & 0.25367 & 0.48743 & 0.28576 \\
\hline $\mathrm{C}$ & 0.24765 & 0.48837 & 0.26115 \\
\hline $\mathrm{C}$ & 0.23321 & 0.48001 & 0.25507 \\
\hline $\mathrm{C}$ & 0.225 & 0.47082 & 0.2732 \\
\hline $\mathrm{C}$ & 0.61543 & 0.86595 & -0.0485 \\
\hline $\mathrm{C}$ & 0.63177 & 0.88001 & -0.0733 \\
\hline $\mathrm{C}$ & 0.64807 & 0.90081 & 0.02487 \\
\hline $\mathrm{C}$ & 0.6585 & 0.91322 & 0.01746 \\
\hline $\mathrm{C}$ & 0.64517 & 0.89668 & -0.0408 \\
\hline $\mathrm{C}$ & 0.65478 & 0.90531 & -0.0603 \\
\hline $\mathrm{C}$ & 0.64776 & 0.91804 & -0.0742 \\
\hline $\mathrm{C}$ & 0.65706 & 0.92647 & -0.0919 \\
\hline $\mathrm{C}$ & 0.67348 & 0.92243 & -0.0959 \\
\hline
\end{tabular}




\begin{tabular}{|c|c|c|c|}
\hline $\mathrm{C}$ & 0.68037 & 0.90934 & -0.0824 \\
\hline $\mathrm{C}$ & 0.67107 & 0.90092 & -0.0647 \\
\hline $\mathrm{N}$ & 0.6826 & 0.93175 & -0.114 \\
\hline $\mathrm{C}$ & 0.69843 & 0.93236 & -0.1157 \\
\hline $\mathrm{C}$ & 0.70648 & 0.94253 & -0.135 \\
\hline $\mathrm{C}$ & 0.72358 & 0.94342 & -0.1354 \\
\hline $\mathrm{C}$ & 0.73155 & 0.95317 & -0.1531 \\
\hline $\mathrm{C}$ & 0.72256 & 0.96223 & -0.1707 \\
\hline $\mathrm{C}$ & 0.70543 & 0.96121 & -0.1704 \\
\hline $\mathrm{C}$ & 0.69745 & 0.95147 & -0.1527 \\
\hline $\mathrm{C}$ & 0.73109 & 0.97278 & -0.1892 \\
\hline $\mathrm{C}$ & 0.74461 & 0.9822 & -0.1818 \\
\hline $\mathrm{C}$ & 0.7528 & 0.99197 & -0.1992 \\
\hline $\mathrm{C}$ & 0.74767 & 0.99254 & -0.2243 \\
\hline $\mathrm{C}$ & 0.7341 & 0.9832 & -0.2317 \\
\hline $\mathrm{C}$ & 0.72586 & 0.97344 & -0.2143 \\
\hline $\mathrm{N}$ & 0.14149 & 0.39323 & 0.49534 \\
\hline $\mathrm{C}$ & 0.54185 & 0.46992 & 0.74985 \\
\hline $\mathrm{C}$ & 0.53381 & 0.48481 & 0.74968 \\
\hline $\mathrm{C}$ & 0.49999 & 0.4581 & 0.75055 \\
\hline $\mathrm{C}$ & 0.48479 & 0.46617 & 0.75023 \\
\hline $\mathrm{C}$ & 0.45814 & 0.4699 & 0.74976 \\
\hline $\mathrm{C}$ & 0.43996 & 0.50003 & 0.74889 \\
\hline $\mathrm{C}$ & 0.43102 & 0.49627 & 0.77055 \\
\hline $\mathrm{C}$ & 0.41395 & 0.49638 & 0.76999 \\
\hline $\mathrm{C}$ & 0.40559 & 0.50053 & 0.74799 \\
\hline $\mathrm{C}$ & 0.41459 & 0.50412 & 0.72616 \\
\hline $\mathrm{C}$ & 0.43165 & 0.50385 & 0.72666 \\
\hline $\mathrm{N}$ & 0.38805 & 0.50047 & 0.74832 \\
\hline $\mathrm{C}$ & 0.37872 & 0.50866 & 0.73315 \\
\hline $\mathrm{C}$ & 0.36076 & 0.50756 & 0.73498 \\
\hline $\mathrm{C}$ & 0.35105 & 0.5182 & 0.72075 \\
\hline $\mathrm{C}$ & 0.33403 & 0.51741 & 0.72219 \\
\hline $\mathrm{C}$ & 0.32639 & 0.50584 & 0.73771 \\
\hline $\mathrm{C}$ & 0.33612 & 0.49512 & 0.75184 \\
\hline $\mathrm{C}$ & 0.35315 & 0.496 & 0.75058 \\
\hline $\mathrm{C}$ & 0.30833 & 0.50495 & 0.73909 \\
\hline $\mathrm{C}$ & 0.29896 & 0.51927 & 0.74104 \\
\hline $\mathrm{C}$ & 0.28194 & 0.51843 & 0.74227 \\
\hline $\mathrm{C}$ & 0.27397 & 0.50327 & 0.74136 \\
\hline $\mathrm{C}$ & 0.28329 & 0.48895 & 0.73937 \\
\hline $\mathrm{C}$ & 0.30032 & 0.48977 & 0.73836 \\
\hline
\end{tabular}




\begin{tabular}{|c|c|c|c|}
\hline $\mathrm{N}$ & 0.25638 & 0.49757 & 0.24233 \\
\hline $\mathrm{H}$ & 0.13819 & 0.38446 & 0.401 \\
\hline $\mathrm{H}$ & 0.16544 & 0.42146 & 0.51987 \\
\hline $\mathrm{H}$ & 0.13558 & 0.42162 & 0.41992 \\
\hline $\mathrm{H}$ & 0.15199 & 0.43617 & 0.3883 \\
\hline $\mathrm{H}$ & 0.19283 & 0.40452 & 0.40462 \\
\hline $\mathrm{H}$ & 0.17655 & 0.39041 & 0.43617 \\
\hline $\mathrm{H}$ & 0.20633 & 0.42415 & 0.38623 \\
\hline $\mathrm{H}$ & 0.23098 & 0.43354 & 0.36491 \\
\hline $\mathrm{H}$ & 0.24483 & 0.44973 & 0.33288 \\
\hline $\mathrm{H}$ & 0.1982 & 0.46708 & 0.30605 \\
\hline $\mathrm{H}$ & 0.18434 & 0.45062 & 0.3378 \\
\hline $\mathrm{H}$ & 0.25035 & 0.4779 & 0.32274 \\
\hline $\mathrm{H}$ & 0.26469 & 0.4939 & 0.29095 \\
\hline $\mathrm{H}$ & 0.22844 & 0.4804 & 0.23615 \\
\hline $\mathrm{H}$ & 0.21406 & 0.46425 & 0.26785 \\
\hline $\mathrm{H}$ & 0.63797 & 0.88468 & -0.0897 \\
\hline $\mathrm{H}$ & 0.66552 & 0.92138 & 0.02924 \\
\hline $\mathrm{H}$ & 0.63516 & 0.9215 & -0.071 \\
\hline $\mathrm{H}$ & 0.65153 & 0.93637 & -0.1024 \\
\hline $\mathrm{H}$ & 0.69281 & 0.90543 & -0.0855 \\
\hline $\mathrm{H}$ & 0.67657 & 0.89099 & -0.0542 \\
\hline $\mathrm{H}$ & 0.70607 & 0.92587 & -0.1025 \\
\hline $\mathrm{H}$ & 0.73081 & 0.93659 & -0.122 \\
\hline $\mathrm{H}$ & 0.74477 & 0.95351 & -0.1531 \\
\hline $\mathrm{H}$ & 0.69817 & 0.96817 & -0.1836 \\
\hline $\mathrm{H}$ & 0.68424 & 0.95103 & -0.1528 \\
\hline $\mathrm{H}$ & 0.74876 & 0.98216 & -0.1626 \\
\hline $\mathrm{H}$ & 0.76312 & 0.9992 & -0.193 \\
\hline $\mathrm{H}$ & 0.73002 & 0.98331 & -0.251 \\
\hline $\mathrm{H}$ & 0.71561 & 0.96617 & -0.2205 \\
\hline $\mathrm{H}$ & 0.55487 & 0.4677 & 0.74983 \\
\hline $\mathrm{H}$ & 0.44511 & 0.46769 & 0.74947 \\
\hline $\mathrm{H}$ & 0.43728 & 0.49323 & 0.78781 \\
\hline $\mathrm{H}$ & 0.4072 & 0.4934 & 0.78683 \\
\hline $\mathrm{H}$ & 0.40855 & 0.50683 & 0.70864 \\
\hline $\mathrm{H}$ & 0.43842 & 0.50663 & 0.70975 \\
\hline $\mathrm{H}$ & 0.38395 & 0.5169 & 0.71946 \\
\hline $\mathrm{H}$ & 0.35664 & 0.52717 & 0.70855 \\
\hline $\mathrm{H}$ & 0.32682 & 0.5257 & 0.71089 \\
\hline $\mathrm{H}$ & 0.33055 & 0.48618 & 0.76412 \\
\hline $\mathrm{H}$ & 0.36033 & 0.48761 & 0.76174 \\
\hline
\end{tabular}




\begin{tabular}{|r|r|r|r|}
\hline $\mathrm{H}$ & 0.30482 & 0.53111 & 0.74188 \\
\hline $\mathrm{H}$ & 0.27494 & 0.52961 & 0.74372 \\
\hline $\mathrm{H}$ & 0.27735 & 0.47715 & 0.73873 \\
\hline $\mathrm{H}$ & 0.30724 & 0.47856 & 0.73663 \\
\hline $\mathrm{N}$ & 0.12417 & 0.37583 & 0.45749 \\
\hline $\mathrm{N}$ & 0.37594 & 0.12406 & -0.0332 \\
\hline $\mathrm{N}$ & 0.5174 & 0.4826 & 0.75 \\
\hline $\mathrm{N}$ & 0.48258 & 0.48258 & 0.75 \\
\hline
\end{tabular}

Table S3. Fractional atomic coordinates for the unit cell of COF-1 with the noninterpenetrated ffc topology.

\begin{tabular}{|c|r|r|r|}
\hline \multicolumn{4}{|l|}{ Space group: $P 4 / \mathrm{m}$} \\
\hline $\mathrm{a}=\mathrm{b}=64.4930 \AA \mathrm{A}, \mathrm{c}=31.1324 \AA$ & \multicolumn{1}{l|}{$\alpha=\gamma=90^{\circ}$} \\
\hline Atom & \multicolumn{1}{|c|}{$\mathrm{x}$} & \multicolumn{1}{c|}{$\mathrm{y}$} & \multicolumn{1}{c|}{$\mathrm{z}$} \\
\hline $\mathrm{C}$ & 0.41316 & 0.49511 & 0.53893 \\
\hline $\mathrm{C}$ & 0.39155 & 0.4939 & 0.5389 \\
\hline $\mathrm{C}$ & 0.31369 & 0.50537 & 0.46135 \\
\hline $\mathrm{C}$ & 0.05357 & 0.49558 & 0.11157 \\
\hline $\mathrm{C}$ & 0.06367 & 0.5135 & 0.12585 \\
\hline $\mathrm{C}$ & 0.07813 & 0.51232 & 0.15912 \\
\hline $\mathrm{C}$ & 0.08256 & 0.49332 & 0.17884 \\
\hline $\mathrm{C}$ & 0.07277 & 0.47523 & 0.16359 \\
\hline $\mathrm{C}$ & 0.05832 & 0.47643 & 0.13028 \\
\hline $\mathrm{N}$ & 0.09697 & 0.4932 & 0.21404 \\
\hline $\mathrm{C}$ & 0.09967 & 0.47827 & 0.24147 \\
\hline $\mathrm{C}$ & 0.11453 & 0.48045 & 0.27721 \\
\hline $\mathrm{C}$ & 0.11588 & 0.46474 & 0.30816 \\
\hline $\mathrm{C}$ & 0.12921 & 0.46679 & 0.34322 \\
\hline $\mathrm{C}$ & 0.14152 & 0.48461 & 0.34801 \\
\hline $\mathrm{C}$ & 0.14039 & 0.5002 & 0.31658 \\
\hline $\mathrm{C}$ & 0.12703 & 0.49816 & 0.28154 \\
\hline $\mathrm{C}$ & 0.15503 & 0.48714 & 0.38625 \\
\hline $\mathrm{C}$ & 0.16604 & 0.47017 & 0.40309 \\
\hline $\mathrm{C}$ & 0.17802 & 0.4724 & 0.44014 \\
\hline $\mathrm{C}$ & 0.17924 & 0.49161 & 0.46084 \\
\hline $\mathrm{C}$ & 0.16859 & 0.50865 & 0.44396 \\
\hline $\mathrm{C}$ & 0.15657 & 0.50645 & 0.40696 \\
\hline $\mathrm{C}$ & 0.29214 & 0.50336 & 0.53879 \\
\hline $\mathrm{C}$ & 0.24582 & 0.49901 & 0.53868 \\
\hline & & & \\
\hline
\end{tabular}




\begin{tabular}{|c|c|c|c|}
\hline $\mathrm{C}$ & 0.22428 & 0.49693 & 0.53862 \\
\hline $\mathrm{C}$ & 0.50136 & 0.01664 & 0.90905 \\
\hline $\mathrm{C}$ & 0.50353 & 0.04386 & 0.03458 \\
\hline $\mathrm{C}$ & 0.50534 & 0.06434 & 0.02188 \\
\hline $\mathrm{C}$ & 0.01052 & 0.49916 & 0.86645 \\
\hline $\mathrm{C}$ & 0.96255 & 0.50305 & 0.07776 \\
\hline $\mathrm{H}$ & 0.42142 & 0.49555 & 0.56916 \\
\hline $\mathrm{H}$ & 0.38323 & 0.49341 & 0.56905 \\
\hline $\mathrm{H}$ & 0.32195 & 0.50613 & 0.43113 \\
\hline $\mathrm{H}$ & 0.06015 & 0.52838 & 0.11157 \\
\hline $\mathrm{H}$ & 0.08566 & 0.52632 & 0.17019 \\
\hline $\mathrm{H}$ & 0.07618 & 0.46017 & 0.17691 \\
\hline $\mathrm{H}$ & 0.05069 & 0.46246 & 0.11926 \\
\hline $\mathrm{H}$ & 0.09039 & 0.46434 & 0.23997 \\
\hline $\mathrm{H}$ & 0.10637 & 0.45098 & 0.30556 \\
\hline $\mathrm{H}$ & 0.1295 & 0.45464 & 0.36715 \\
\hline $\mathrm{H}$ & 0.14989 & 0.51397 & 0.31903 \\
\hline $\mathrm{H}$ & 0.12639 & 0.51049 & 0.25804 \\
\hline $\mathrm{H}$ & 0.1654 & 0.45518 & 0.38758 \\
\hline $\mathrm{H}$ & 0.18623 & 0.45917 & 0.45302 \\
\hline $\mathrm{H}$ & 0.16945 & 0.52355 & 0.45975 \\
\hline $\mathrm{H}$ & 0.14809 & 0.51975 & 0.39517 \\
\hline $\mathrm{H}$ & 0.28496 & 0.50268 & 0.5699 \\
\hline $\mathrm{H}$ & 0.253 & 0.49977 & 0.56977 \\
\hline $\mathrm{H}$ & 0.2164 & 0.49629 & 0.56923 \\
\hline $\mathrm{H}$ & 0.50659 & 0.07792 & 0.04208 \\
\hline $\mathrm{H}$ & 0.02021 & 0.4984 & 0.83816 \\
\hline $\mathrm{C}$ & 0.49702 & 0.55302 & 0.5 \\
\hline $\mathrm{C}$ & 0.47835 & 0.54173 & 0.5 \\
\hline $\mathrm{C}$ & 0.45893 & 0.55086 & 0.5 \\
\hline $\mathrm{C}$ & 0.42405 & 0.49573 & 0.5 \\
\hline $\mathrm{C}$ & 0.38069 & 0.49337 & 0.5 \\
\hline $\mathrm{N}$ & 0.35862 & 0.49203 & 0.5 \\
\hline $\mathrm{C}$ & 0.28068 & 0.50229 & 0.5 \\
\hline $\mathrm{C}$ & 0.25727 & 0.50007 & 0.5 \\
\hline $\mathrm{C}$ & 0.21314 & 0.49573 & 0.5 \\
\hline $\mathrm{C}$ & 0.45611 & 0.51684 & 0.5 \\
\hline $\mathrm{C}$ & 0.44485 & 0.53511 & 0.5 \\
\hline $\mathrm{N}$ & 0.47672 & 0.5208 & 0.5 \\
\hline $\mathrm{N}$ & 0.19076 & 0.49362 & 0.5 \\
\hline $\mathrm{C}$ & 0.34708 & 0.50844 & 0.5 \\
\hline $\mathrm{C}$ & 0.32451 & 0.50635 & 0.5 \\
\hline
\end{tabular}




\begin{tabular}{|r|r|r|r|}
$\mathrm{H}$ & 0.45524 & 0.56722 & 0.5 \\
\hline $\mathrm{H}$ & 0.42818 & 0.53694 & 0.5 \\
\hline $\mathrm{H}$ & 0.35391 & 0.52378 & 0.5 \\
\hline $\mathrm{N}$ & 0.96883 & 0.50242 & 0 \\
\hline $\mathrm{N}$ & 0 & 0.5 & 0.06456 \\
\hline
\end{tabular}

Table S4. Fractional atomic coordinates for the unit cell of COF-1 with the noninterpenetrated tbo topology.

\begin{tabular}{|c|c|c|c|}
\hline \multicolumn{4}{|c|}{ Space group: $P \mathrm{~m}-3$} \\
\hline \multicolumn{4}{|c|}{$\mathrm{a}=\mathrm{b}=\mathrm{c}=95.3162 \AA, \alpha=\beta=\gamma=90^{\circ}$} \\
\hline Atom & $\mathrm{x}$ & $\mathrm{y}$ & $\mathrm{z}$ \\
\hline $\mathrm{C}$ & 0.27922 & 0.72869 & 0.46422 \\
\hline $\mathrm{C}$ & 0.27631 & 0.71483 & 0.46151 \\
\hline $\mathrm{C}$ & 0.28318 & 0.70793 & 0.45092 \\
\hline $\mathrm{C}$ & 0.29311 & 0.71471 & 0.44296 \\
\hline $\mathrm{C}$ & 0.29602 & 0.72866 & 0.44572 \\
\hline $\mathrm{C}$ & 0.28914 & 0.73555 & 0.45631 \\
\hline $\mathrm{N}$ & 0.29944 & 0.70726 & 0.43185 \\
\hline $\mathrm{C}$ & 0.31037 & 0.71089 & 0.42504 \\
\hline $\mathrm{C}$ & 0.31545 & 0.70248 & 0.41344 \\
\hline $\mathrm{C}$ & 0.3272 & 0.70673 & 0.4062 \\
\hline $\mathrm{C}$ & 0.33201 & 0.69914 & 0.39498 \\
\hline $\mathrm{C}$ & 0.32517 & 0.6871 & 0.39077 \\
\hline $\mathrm{C}$ & 0.31346 & 0.68278 & 0.3981 \\
\hline $\mathrm{C}$ & 0.30864 & 0.6904 & 0.40932 \\
\hline $\mathrm{C}$ & 0.3301 & 0.67917 & 0.37867 \\
\hline $\mathrm{C}$ & 0.33466 & 0.68602 & 0.36677 \\
\hline $\mathrm{C}$ & 0.33911 & 0.67858 & 0.3553 \\
\hline $\mathrm{C}$ & 0.33913 & 0.66412 & 0.35549 \\
\hline $\mathrm{C}$ & 0.33464 & 0.65726 & 0.3674 \\
\hline $\mathrm{C}$ & 0.33016 & 0.6647 & 0.37886 \\
\hline $\mathrm{C}$ & 0.77183 & 0.22137 & 0.46421 \\
\hline $\mathrm{C}$ & 0.76518 & 0.21134 & 0.45627 \\
\hline $\mathrm{C}$ & 0.77224 & 0.20464 & 0.44569 \\
\hline $\mathrm{C}$ & 0.78599 & 0.20791 & 0.44281 \\
\hline $\mathrm{C}$ & 0.79275 & 0.21778 & 0.45102 \\
\hline $\mathrm{C}$ & 0.78568 & 0.22448 & 0.46161 \\
\hline $\mathrm{N}$ & 0.79259 & 0.20092 & 0.43155 \\
\hline
\end{tabular}




\begin{tabular}{|c|c|c|c|}
\hline $\mathrm{C}$ & 0.80418 & 0.20429 & 0.42577 \\
\hline $\mathrm{C}$ & 0.80962 & 0.19631 & 0.41403 \\
\hline $\mathrm{C}$ & 0.82184 & 0.20055 & 0.40762 \\
\hline $\mathrm{C}$ & 0.82702 & 0.19336 & 0.39629 \\
\hline $\mathrm{C}$ & 0.82008 & 0.18175 & 0.39115 \\
\hline $\mathrm{C}$ & 0.8079 & 0.17744 & 0.39766 \\
\hline $\mathrm{C}$ & 0.80271 & 0.18464 & 0.40898 \\
\hline $\mathrm{C}$ & 0.82543 & 0.17427 & 0.37895 \\
\hline $\mathrm{C}$ & 0.83079 & 0.18155 & 0.36764 \\
\hline $\mathrm{C}$ & 0.83564 & 0.17453 & 0.35607 \\
\hline $\mathrm{C}$ & 0.83528 & 0.16009 & 0.35556 \\
\hline $\mathrm{C}$ & 0.82999 & 0.1528 & 0.36687 \\
\hline $\mathrm{C}$ & 0.82511 & 0.15981 & 0.37844 \\
\hline $\mathrm{C}$ & 0.27165 & 0.23111 & 0.96423 \\
\hline $\mathrm{C}$ & 0.26931 & 0.21715 & 0.96147 \\
\hline $\mathrm{C}$ & 0.27664 & 0.21052 & 0.95102 \\
\hline $\mathrm{C}$ & 0.28646 & 0.21767 & 0.94325 \\
\hline $\mathrm{C}$ & 0.28875 & 0.23174 & 0.94602 \\
\hline $\mathrm{C}$ & 0.28141 & 0.23836 & 0.95646 \\
\hline $\mathrm{N}$ & 0.2934 & 0.21042 & 0.93238 \\
\hline $\mathrm{C}$ & 0.30434 & 0.21447 & 0.92583 \\
\hline $\mathrm{C}$ & 0.31018 & 0.20612 & 0.91454 \\
\hline $\mathrm{C}$ & 0.32197 & 0.21079 & 0.90764 \\
\hline $\mathrm{C}$ & 0.32756 & 0.2032 & 0.89678 \\
\hline $\mathrm{C}$ & 0.32147 & 0.19075 & 0.8926 \\
\hline $\mathrm{C}$ & 0.3097 & 0.18605 & 0.89958 \\
\hline $\mathrm{C}$ & 0.3041 & 0.19366 & 0.91043 \\
\hline $\mathrm{C}$ & 0.32729 & 0.18277 & 0.88094 \\
\hline $\mathrm{C}$ & 0.33204 & 0.18954 & 0.86906 \\
\hline $\mathrm{C}$ & 0.33739 & 0.18203 & 0.85803 \\
\hline $\mathrm{C}$ & 0.33812 & 0.16761 & 0.85863 \\
\hline $\mathrm{C}$ & 0.33343 & 0.16084 & 0.87051 \\
\hline $\mathrm{C}$ & 0.32806 & 0.16833 & 0.88155 \\
\hline $\mathrm{C}$ & 0.78119 & 0.72086 & 0.96419 \\
\hline $\mathrm{C}$ & 0.77488 & 0.71034 & 0.95661 \\
\hline $\mathrm{C}$ & 0.78197 & 0.70389 & 0.94589 \\
\hline $\mathrm{C}$ & 0.79539 & 0.70791 & 0.94251 \\
\hline $\mathrm{C}$ & 0.80186 & 0.71825 & 0.95038 \\
\hline $\mathrm{C}$ & 0.79476 & 0.72469 & 0.96112 \\
\hline $\mathrm{N}$ & 0.80196 & 0.70125 & 0.93103 \\
\hline $\mathrm{C}$ & 0.81305 & 0.7053 & 0.92474 \\
\hline $\mathrm{C}$ & 0.8184 & 0.69763 & 0.91275 \\
\hline
\end{tabular}




\begin{tabular}{|c|c|c|c|}
\hline $\mathrm{C}$ & 0.82989 & 0.70272 & 0.90563 \\
\hline $\mathrm{C}$ & 0.83487 & 0.69588 & 0.89399 \\
\hline $\mathrm{C}$ & 0.82848 & 0.6838 & 0.88926 \\
\hline $\mathrm{C}$ & 0.81708 & 0.67862 & 0.89651 \\
\hline $\mathrm{C}$ & 0.81208 & 0.68547 & 0.90813 \\
\hline $\mathrm{C}$ & 0.83354 & 0.67673 & 0.87668 \\
\hline $\mathrm{C}$ & 0.83754 & 0.68439 & 0.86508 \\
\hline $\mathrm{C}$ & 0.84205 & 0.67776 & 0.85315 \\
\hline $\mathrm{C}$ & 0.84269 & 0.66333 & 0.85257 \\
\hline $\mathrm{C}$ & 0.83877 & 0.65566 & 0.86418 \\
\hline $\mathrm{C}$ & 0.83423 & 0.66229 & 0.87611 \\
\hline $\mathrm{C}$ & 0.25316 & 0.45733 & 0.74316 \\
\hline $\mathrm{C}$ & 0.2548 & 0.47089 & 0.73906 \\
\hline $\mathrm{N}$ & 0.24635 & 0.4793 & 0.74632 \\
\hline $\mathrm{C}$ & 0.23928 & 0.47089 & 0.75492 \\
\hline $\mathrm{C}$ & 0.24334 & 0.45733 & 0.75319 \\
\hline $\mathrm{C}$ & 0.22891 & 0.47507 & 0.76416 \\
\hline $\mathrm{C}$ & 0.22531 & 0.48891 & 0.76654 \\
\hline $\mathrm{C}$ & 0.21467 & 0.50702 & 0.77512 \\
\hline $\mathrm{C}$ & 0.26381 & 0.47507 & 0.72848 \\
\hline $\mathrm{C}$ & 0.26611 & 0.48891 & 0.72483 \\
\hline $\mathrm{C}$ & 0.27449 & 0.49298 & 0.71403 \\
\hline $\mathrm{C}$ & 0.74206 & 0.0427 & 0.23828 \\
\hline $\mathrm{C}$ & 0.73819 & 0.02912 & 0.23629 \\
\hline $\mathrm{N}$ & 0.745 & 0.0207 & 0.24509 \\
\hline $\mathrm{C}$ & 0.75309 & 0.02912 & 0.25273 \\
\hline $\mathrm{C}$ & 0.75149 & 0.0427 & 0.24868 \\
\hline $\mathrm{C}$ & 0.76173 & 0.02494 & 0.2636 \\
\hline $\mathrm{C}$ & 0.76394 & 0.01109 & 0.26729 \\
\hline $\mathrm{C}$ & 0.77194 & 0.99298 & 0.27838 \\
\hline $\mathrm{C}$ & 0.72821 & 0.02494 & 0.22662 \\
\hline $\mathrm{C}$ & 0.72478 & 0.01109 & 0.22401 \\
\hline $\mathrm{C}$ & 0.71459 & 0.00702 & 0.21491 \\
\hline $\mathrm{N}$ & 0.84016 & 0.15284 & 0.34353 \\
\hline $\mathrm{H}$ & 0.2686 & 0.70943 & 0.46749 \\
\hline $\mathrm{H}$ & 0.28071 & 0.69726 & 0.44885 \\
\hline $\mathrm{H}$ & 0.3033 & 0.73442 & 0.4396 \\
\hline $\mathrm{H}$ & 0.29138 & 0.74631 & 0.45822 \\
\hline $\mathrm{H}$ & 0.31601 & 0.72009 & 0.42776 \\
\hline $\mathrm{H}$ & 0.33263 & 0.71599 & 0.40921 \\
\hline $\mathrm{H}$ & 0.34115 & 0.70263 & 0.38965 \\
\hline $\mathrm{H}$ & 0.30787 & 0.67366 & 0.39501 \\
\hline
\end{tabular}




\begin{tabular}{|c|c|c|c|}
\hline $\mathrm{H}$ & 0.29953 & 0.68687 & 0.41468 \\
\hline $\mathrm{H}$ & 0.33454 & 0.69715 & 0.36622 \\
\hline $\mathrm{H}$ & 0.34238 & 0.68409 & 0.34618 \\
\hline $\mathrm{H}$ & 0.33468 & 0.64613 & 0.36782 \\
\hline $\mathrm{H}$ & 0.32689 & 0.65913 & 0.38794 \\
\hline $\mathrm{H}$ & 0.75449 & 0.20882 & 0.4582 \\
\hline $\mathrm{H}$ & 0.76693 & 0.19699 & 0.43956 \\
\hline $\mathrm{H}$ & 0.80345 & 0.22034 & 0.44944 \\
\hline $\mathrm{H}$ & 0.79095 & 0.23215 & 0.46774 \\
\hline $\mathrm{H}$ & 0.80983 & 0.21329 & 0.42908 \\
\hline $\mathrm{H}$ & 0.82736 & 0.20949 & 0.41135 \\
\hline $\mathrm{H}$ & 0.83652 & 0.19683 & 0.39161 \\
\hline $\mathrm{H}$ & 0.80223 & 0.16863 & 0.39384 \\
\hline $\mathrm{H}$ & 0.79324 & 0.18113 & 0.4137 \\
\hline $\mathrm{H}$ & 0.83098 & 0.19269 & 0.36764 \\
\hline $\mathrm{H}$ & 0.83952 & 0.18037 & 0.34741 \\
\hline $\mathrm{H}$ & 0.82973 & 0.14166 & 0.36675 \\
\hline $\mathrm{H}$ & 0.82121 & 0.15391 & 0.38705 \\
\hline $\mathrm{H}$ & 0.26174 & 0.21145 & 0.96734 \\
\hline $\mathrm{H}$ & 0.27466 & 0.19975 & 0.94894 \\
\hline $\mathrm{H}$ & 0.29592 & 0.23777 & 0.94004 \\
\hline $\mathrm{H}$ & 0.2832 & 0.24919 & 0.95842 \\
\hline $\mathrm{H}$ & 0.30948 & 0.22395 & 0.92858 \\
\hline $\mathrm{H}$ & 0.32686 & 0.22035 & 0.91064 \\
\hline $\mathrm{H}$ & 0.33674 & 0.207 & 0.89172 \\
\hline $\mathrm{H}$ & 0.30467 & 0.17659 & 0.89649 \\
\hline $\mathrm{H}$ & 0.29496 & 0.18981 & 0.91553 \\
\hline $\mathrm{H}$ & 0.33139 & 0.20063 & 0.8682 \\
\hline $\mathrm{H}$ & 0.3408 & 0.18746 & 0.84891 \\
\hline $\mathrm{H}$ & 0.33402 & 0.14973 & 0.87125 \\
\hline $\mathrm{H}$ & 0.32466 & 0.16284 & 0.89063 \\
\hline $\mathrm{H}$ & 0.76441 & 0.70727 & 0.9589 \\
\hline $\mathrm{H}$ & 0.7769 & 0.69589 & 0.94002 \\
\hline $\mathrm{H}$ & 0.81233 & 0.7214 & 0.9484 \\
\hline $\mathrm{H}$ & 0.79977 & 0.73275 & 0.96696 \\
\hline $\mathrm{H}$ & 0.81824 & 0.71467 & 0.92775 \\
\hline $\mathrm{H}$ & 0.83496 & 0.71205 & 0.90902 \\
\hline $\mathrm{H}$ & 0.84379 & 0.70002 & 0.88873 \\
\hline $\mathrm{H}$ & 0.81184 & 0.66942 & 0.89303 \\
\hline $\mathrm{H}$ & 0.80319 & 0.6813 & 0.9134 \\
\hline $\mathrm{H}$ & 0.83692 & 0.69552 & 0.86512 \\
\hline $\mathrm{H}$ & 0.84486 & 0.68388 & 0.84427 \\
\hline
\end{tabular}




\begin{tabular}{|r|r|r|r|}
\hline $\mathrm{H}$ & 0.8393 & 0.64454 & 0.86401 \\
\hline $\mathrm{H}$ & 0.83139 & 0.65612 & 0.88495 \\
\hline $\mathrm{H}$ & 0.25845 & 0.44832 & 0.7393 \\
\hline $\mathrm{H}$ & 0.2396 & 0.44833 & 0.75857 \\
\hline $\mathrm{H}$ & 0.20765 & 0.5135 & 0.78084 \\
\hline $\mathrm{H}$ & 0.28008 & 0.4865 & 0.7069 \\
\hline $\mathrm{H}$ & 0.73845 & 0.05172 & 0.23284 \\
\hline $\mathrm{H}$ & 0.75656 & 0.05172 & 0.25281 \\
\hline $\mathrm{H}$ & 0.77726 & 0.9865 & 0.28571 \\
\hline $\mathrm{H}$ & 0.70785 & 0.01351 & 0.20885 \\
\hline $\mathrm{N}$ & 0.76117 & 0.23184 & 0.5 \\
\hline $\mathrm{N}$ & 0.73147 & 0.26088 & 0.5 \\
\hline $\mathrm{N}$ & 0.26047 & 0.75895 & 0 \\
\hline $\mathrm{N}$ & 0.22968 & 0.73106 & 0 \\
\hline $\mathrm{N}$ & 0.34357 & 0.65643 & 0.34357 \\
\hline
\end{tabular}

Table S5. Fractional atomic coordinates for the unit cell of COF-1 with the interpenetrated pto topology.

\begin{tabular}{|c|c|c|c|}
\hline \multicolumn{4}{|c|}{ Space group: $P-421 \mathrm{c}$} \\
\hline \multicolumn{4}{|c|}{$\mathrm{a}=\mathrm{b}=\mathrm{c}=57.3636 \AA, \alpha=\beta=\gamma=90^{\circ}$} \\
\hline Atom & $\mathrm{x}$ & $\mathrm{y}$ & $\mathrm{Z}$ \\
\hline $\mathrm{C}$ & 0.98568 & 0.5498 & 0.12583 \\
\hline $\mathrm{N}$ & 1.00209 & 0.53325 & 0.12979 \\
\hline $\mathrm{C}$ & 0.02597 & 0.54435 & 0.12738 \\
\hline $\mathrm{C}$ & 0.02265 & 0.56775 & 0.12193 \\
\hline $\mathrm{C}$ & 0.99593 & 0.57119 & 0.12087 \\
\hline $\mathrm{C}$ & 0.04798 & 0.53382 & 0.13016 \\
\hline $\mathrm{C}$ & 0.06898 & 0.5487 & 0.12812 \\
\hline $\mathrm{C}$ & 0.07554 & 0.56331 & 0.14831 \\
\hline $\mathrm{C}$ & 0.09541 & 0.57725 & 0.14613 \\
\hline $\mathrm{C}$ & 0.10903 & 0.57663 & 0.12392 \\
\hline $\mathrm{C}$ & 0.10227 & 0.56203 & 0.10359 \\
\hline $\mathrm{C}$ & 0.08241 & 0.54815 & 0.10577 \\
\hline $\mathrm{N}$ & 0.12862 & 0.59209 & 0.1222 \\
\hline $\mathrm{C}$ & 0.14548 & 0.59094 & 0.10569 \\
\hline $\mathrm{C}$ & 0.16335 & 0.60937 & 0.10474 \\
\hline $\mathrm{C}$ & 0.17949 & 0.60971 & 0.08462 \\
\hline $\mathrm{C}$ & 0.19518 & 0.6282 & 0.08217 \\
\hline $\mathrm{C}$ & 0.19512 & 0.64654 & 0.09996 \\
\hline
\end{tabular}




\begin{tabular}{|c|c|c|c|}
\hline $\mathrm{C}$ & 0.17947 & 0.64573 & 0.12058 \\
\hline $\mathrm{C}$ & 0.16372 & 0.62735 & 0.12294 \\
\hline $\mathrm{C}$ & 0.2103 & 0.66713 & 0.09637 \\
\hline $\mathrm{C}$ & 0.21298 & 0.67711 & 0.07202 \\
\hline $\mathrm{C}$ & 0.22564 & 0.6977 & 0.06895 \\
\hline $\mathrm{C}$ & 0.23563 & 0.70872 & 0.09017 \\
\hline $\mathrm{C}$ & 0.23348 & 0.69852 & 0.11439 \\
\hline $\mathrm{C}$ & 0.221 & 0.67785 & 0.11747 \\
\hline $\mathrm{C}$ & 0.01123 & 0.54965 & 0.63293 \\
\hline $\mathrm{N}$ & -0.0051 & 0.53281 & 0.6301 \\
\hline $\mathrm{C}$ & 0.97099 & 0.54403 & 0.63142 \\
\hline $\mathrm{C}$ & 0.97422 & 0.56766 & 0.63557 \\
\hline $\mathrm{C}$ & 0.00088 & 0.57121 & 0.63663 \\
\hline $\mathrm{C}$ & 0.94908 & 0.53374 & 0.6271 \\
\hline $\mathrm{C}$ & 0.92917 & 0.5495 & 0.62209 \\
\hline $\mathrm{C}$ & 0.92781 & 0.56111 & 0.59861 \\
\hline $\mathrm{C}$ & 0.91012 & 0.57725 & 0.59418 \\
\hline $\mathrm{C}$ & 0.89347 & 0.58195 & 0.61301 \\
\hline $\mathrm{C}$ & 0.8947 & 0.57009 & 0.63657 \\
\hline $\mathrm{C}$ & 0.91248 & 0.55391 & 0.64101 \\
\hline $\mathrm{N}$ & 0.87647 & 0.59962 & 0.60769 \\
\hline $\mathrm{C}$ & 0.85773 & 0.60414 & 0.62066 \\
\hline $\mathrm{C}$ & 0.84227 & 0.62333 & 0.61274 \\
\hline $\mathrm{C}$ & 0.82119 & 0.62693 & 0.62568 \\
\hline $\mathrm{C}$ & 0.80624 & 0.64492 & 0.61829 \\
\hline $\mathrm{C}$ & 0.81216 & 0.6597 & 0.59787 \\
\hline $\mathrm{C}$ & 0.83337 & 0.65618 & 0.58506 \\
\hline $\mathrm{C}$ & 0.84828 & 0.63817 & 0.59239 \\
\hline $\mathrm{C}$ & 0.79609 & 0.67851 & 0.58976 \\
\hline $\mathrm{C}$ & 0.78395 & 0.69171 & 0.60805 \\
\hline $\mathrm{C}$ & 0.76807 & 0.70874 & 0.60042 \\
\hline $\mathrm{C}$ & 0.76404 & 0.7129 & 0.5744 \\
\hline $\mathrm{C}$ & 0.77644 & 0.70013 & 0.55609 \\
\hline $\mathrm{C}$ & 0.79235 & 0.68306 & 0.56368 \\
\hline $\mathrm{C}$ & 0.23319 & 0.98098 & 0.38198 \\
\hline $\mathrm{N}$ & 0.23294 & 0.99893 & 0.36515 \\
\hline $\mathrm{C}$ & 0.23273 & 0.02166 & 0.37966 \\
\hline $\mathrm{C}$ & 0.23269 & 0.01607 & 0.40541 \\
\hline $\mathrm{C}$ & 0.23317 & 0.98917 & 0.40687 \\
\hline $\mathrm{C}$ & 0.23316 & 0.04467 & 0.3703 \\
\hline $\mathrm{C}$ & 0.23362 & 0.06401 & 0.38905 \\
\hline $\mathrm{C}$ & 0.25411 & 0.06916 & 0.40249 \\
\hline
\end{tabular}




\begin{tabular}{|c|c|c|c|}
\hline $\mathrm{C}$ & 0.25443 & 0.08746 & 0.42006 \\
\hline $\mathrm{C}$ & 0.2344 & 0.10084 & 0.4244 \\
\hline $\mathrm{C}$ & 0.21392 & 0.09581 & 0.41062 \\
\hline $\mathrm{C}$ & 0.21356 & 0.07741 & 0.39318 \\
\hline $\mathrm{N}$ & 0.2359 & 0.11986 & 0.44212 \\
\hline $\mathrm{C}$ & 0.21848 & 0.13066 & 0.45265 \\
\hline $\mathrm{C}$ & 0.22262 & 0.15056 & 0.46985 \\
\hline $\mathrm{C}$ & 0.20365 & 0.16204 & 0.48111 \\
\hline $\mathrm{C}$ & 0.20726 & 0.18136 & 0.49688 \\
\hline $\mathrm{C}$ & 0.22993 & 0.18974 & 0.50134 \\
\hline $\mathrm{C}$ & 0.24891 & 0.17785 & 0.49048 \\
\hline $\mathrm{C}$ & 0.24531 & 0.15846 & 0.47493 \\
\hline $\mathrm{C}$ & 0.23391 & 0.21073 & 0.51727 \\
\hline $\mathrm{C}$ & 0.22054 & 0.2147 & 0.53936 \\
\hline $\mathrm{C}$ & 0.22528 & 0.23378 & 0.55509 \\
\hline $\mathrm{C}$ & 0.24358 & 0.24901 & 0.5491 \\
\hline $\mathrm{C}$ & 0.25655 & 0.2455 & 0.52681 \\
\hline $\mathrm{C}$ & 0.25163 & 0.22665 & 0.51088 \\
\hline $\mathrm{C}$ & 0.22476 & 0.01624 & 0.27209 \\
\hline $\mathrm{N}$ & 0.22723 & -0.0017 & 0.28868 \\
\hline $\mathrm{C}$ & 0.22441 & 0.97562 & 0.27444 \\
\hline $\mathrm{C}$ & 0.21991 & 0.98123 & 0.24917 \\
\hline $\mathrm{C}$ & 0.22013 & 0.00812 & 0.24769 \\
\hline $\mathrm{C}$ & 0.22646 & 0.95263 & 0.28346 \\
\hline $\mathrm{C}$ & 0.22579 & 0.93351 & 0.26449 \\
\hline $\mathrm{C}$ & 0.24519 & 0.92938 & 0.24891 \\
\hline $\mathrm{C}$ & 0.24479 & 0.91126 & 0.23117 \\
\hline $\mathrm{C}$ & 0.22518 & 0.89689 & 0.22896 \\
\hline $\mathrm{C}$ & 0.20552 & 0.9013 & 0.24434 \\
\hline $\mathrm{C}$ & 0.20588 & 0.91953 & 0.26203 \\
\hline $\mathrm{N}$ & 0.22605 & 0.87826 & 0.21075 \\
\hline $\mathrm{C}$ & 0.21232 & 0.8603 & 0.20976 \\
\hline $\mathrm{C}$ & 0.21618 & 0.84209 & 0.19039 \\
\hline $\mathrm{C}$ & 0.20368 & 0.82113 & 0.19177 \\
\hline $\mathrm{C}$ & 0.20813 & 0.80319 & 0.17439 \\
\hline $\mathrm{C}$ & 0.22512 & 0.80598 & 0.15528 \\
\hline $\mathrm{C}$ & 0.23718 & 0.82722 & 0.15355 \\
\hline $\mathrm{C}$ & 0.23279 & 0.84509 & 0.17094 \\
\hline $\mathrm{C}$ & 0.23072 & 0.78664 & 0.13758 \\
\hline $\mathrm{C}$ & 0.21295 & 0.77262 & 0.12734 \\
\hline $\mathrm{C}$ & 0.21838 & 0.75433 & 0.1108 \\
\hline $\mathrm{C}$ & 0.24165 & 0.74975 & 0.10418 \\
\hline
\end{tabular}




\begin{tabular}{|c|c|c|c|}
\hline $\mathrm{C}$ & 0.2594 & 0.76372 & 0.11441 \\
\hline $\mathrm{C}$ & 0.25401 & 0.78197 & 0.13096 \\
\hline $\mathrm{C}$ & 0.54848 & 0.26869 & 0.80931 \\
\hline $\mathrm{N}$ & 0.5332 & 0.26803 & 0.82902 \\
\hline $\mathrm{C}$ & 0.54646 & 0.26523 & -0.14979 \\
\hline $\mathrm{C}$ & 0.56989 & 0.26417 & -0.15614 \\
\hline $\mathrm{C}$ & 0.57115 & 0.2662 & 0.81798 \\
\hline $\mathrm{C}$ & 0.53805 & 0.26436 & -0.12453 \\
\hline $\mathrm{C}$ & 0.55538 & 0.26282 & -0.10359 \\
\hline $\mathrm{C}$ & 0.56043 & 0.28251 & -0.08874 \\
\hline $\mathrm{C}$ & 0.57753 & 0.28136 & -0.06981 \\
\hline $\mathrm{C}$ & 0.59015 & 0.26079 & -0.06594 \\
\hline $\mathrm{C}$ & 0.58473 & 0.24088 & -0.08047 \\
\hline $\mathrm{C}$ & 0.56736 & 0.2419 & -0.09913 \\
\hline $\mathrm{N}$ & 0.60808 & 0.26066 & -0.04691 \\
\hline $\mathrm{C}$ & 0.62655 & 0.24758 & -0.04742 \\
\hline $\mathrm{C}$ & 0.64409 & 0.24934 & -0.027 \\
\hline $\mathrm{C}$ & 0.66639 & 0.24 & -0.03079 \\
\hline $\mathrm{C}$ & 0.68346 & 0.24232 & -0.01195 \\
\hline $\mathrm{C}$ & 0.6785 & 0.25382 & 0.01115 \\
\hline $\mathrm{C}$ & 0.65594 & 0.26268 & 0.01507 \\
\hline $\mathrm{C}$ & 0.63896 & 0.2606 & -0.00386 \\
\hline $\mathrm{C}$ & 0.69688 & 0.25689 & 0.03087 \\
\hline $\mathrm{C}$ & 0.71399 & 0.23968 & 0.03471 \\
\hline $\mathrm{C}$ & 0.73138 & 0.2428 & 0.05302 \\
\hline $\mathrm{C}$ & 0.73206 & 0.26317 & 0.06781 \\
\hline $\mathrm{C}$ & 0.71515 & 0.28043 & 0.06395 \\
\hline $\mathrm{C}$ & 0.69761 & 0.27723 & 0.04587 \\
\hline $\mathrm{C}$ & 0.44794 & 0.26537 & -0.15582 \\
\hline $\mathrm{N}$ & 0.46319 & 0.26825 & -0.17531 \\
\hline $\mathrm{C}$ & 0.45002 & 0.26791 & 0.80321 \\
\hline $\mathrm{C}$ & 0.42672 & 0.26457 & 0.80909 \\
\hline $\mathrm{C}$ & 0.4254 & 0.26301 & -0.16497 \\
\hline $\mathrm{C}$ & 0.45843 & 0.27113 & 0.77821 \\
\hline $\mathrm{C}$ & 0.44138 & 0.27175 & 0.75699 \\
\hline $\mathrm{C}$ & 0.42761 & 0.29158 & 0.75299 \\
\hline $\mathrm{C}$ & 0.41192 & 0.29227 & 0.73262 \\
\hline $\mathrm{C}$ & 0.40999 & 0.27334 & 0.71586 \\
\hline $\mathrm{C}$ & 0.42363 & 0.2533 & 0.72014 \\
\hline $\mathrm{C}$ & 0.43925 & 0.25259 & 0.74056 \\
\hline $\mathrm{N}$ & 0.39327 & 0.27477 & 0.69556 \\
\hline $\mathrm{C}$ & 0.39279 & 0.26185 & 0.67519 \\
\hline
\end{tabular}




\begin{tabular}{|c|c|c|c|}
\hline $\mathrm{C}$ & 0.37371 & 0.26432 & 0.65654 \\
\hline $\mathrm{C}$ & 0.37264 & 0.24926 & 0.63548 \\
\hline $\mathrm{C}$ & 0.3539 & 0.25041 & 0.61848 \\
\hline $\mathrm{C}$ & 0.33595 & 0.26672 & 0.62225 \\
\hline $\mathrm{C}$ & 0.33738 & 0.28219 & 0.643 \\
\hline $\mathrm{C}$ & 0.35606 & 0.281 & 0.65998 \\
\hline $\mathrm{C}$ & 0.31509 & 0.26684 & 0.60559 \\
\hline $\mathrm{C}$ & 0.30448 & 0.24577 & 0.5984 \\
\hline $\mathrm{C}$ & 0.2835 & 0.24585 & 0.58493 \\
\hline $\mathrm{C}$ & 0.27269 & 0.26701 & 0.5784 \\
\hline $\mathrm{C}$ & 0.28379 & 0.28806 & 0.58469 \\
\hline $\mathrm{C}$ & 0.30476 & 0.28799 & 0.59817 \\
\hline $\mathrm{N}$ & 0.24998 & 0.26717 & 0.56658 \\
\hline $\mathrm{N}$ & 0.74955 & 0.26606 & 0.08727 \\
\hline $\mathrm{H}$ & 0.03581 & 0.58098 & 0.11889 \\
\hline $\mathrm{H}$ & 0.98736 & 0.58762 & 0.11698 \\
\hline $\mathrm{H}$ & 0.06524 & 0.56394 & 0.16564 \\
\hline $\mathrm{H}$ & 0.10021 & 0.58864 & 0.16176 \\
\hline $\mathrm{H}$ & 0.1118 & 0.5616 & 0.08581 \\
\hline $\mathrm{H}$ & 0.07736 & 0.53705 & 0.08997 \\
\hline $\mathrm{H}$ & 0.14633 & 0.57697 & 0.09186 \\
\hline $\mathrm{H}$ & 0.17955 & 0.59598 & 0.07042 \\
\hline $\mathrm{H}$ & 0.2072 & 0.62831 & 0.06622 \\
\hline $\mathrm{H}$ & 0.1788 & 0.65977 & 0.13439 \\
\hline $\mathrm{H}$ & 0.15149 & 0.62754 & 0.1387 \\
\hline $\mathrm{H}$ & 0.20458 & 0.66949 & 0.0555 \\
\hline $\mathrm{H}$ & 0.22699 & 0.70551 & 0.0502 \\
\hline $\mathrm{H}$ & 0.24132 & 0.70674 & 0.13091 \\
\hline $\mathrm{H}$ & 0.21948 & 0.67038 & 0.13639 \\
\hline $\mathrm{H}$ & 0.96101 & 0.58104 & 0.63725 \\
\hline $\mathrm{H}$ & 0.00937 & 0.58789 & 0.63921 \\
\hline $\mathrm{H}$ & 0.94073 & 0.55795 & 0.58392 \\
\hline $\mathrm{H}$ & 0.9095 & 0.58637 & 0.57608 \\
\hline $\mathrm{H}$ & 0.88251 & 0.57359 & 0.65182 \\
\hline $\mathrm{H}$ & 0.91355 & 0.54521 & 0.65933 \\
\hline $\mathrm{H}$ & 0.85291 & 0.59372 & 0.63704 \\
\hline $\mathrm{H}$ & 0.8162 & 0.6157 & 0.64138 \\
\hline $\mathrm{H}$ & 0.78985 & 0.647 & 0.6283 \\
\hline $\mathrm{H}$ & 0.83854 & 0.6675 & 0.5695 \\
\hline $\mathrm{H}$ & 0.86452 & 0.63592 & 0.58215 \\
\hline $\mathrm{H}$ & 0.78678 & 0.68889 & 0.62827 \\
\hline $\mathrm{H}$ & 0.75879 & 0.71865 & 0.61479 \\
\hline
\end{tabular}




\begin{tabular}{|c|c|c|c|}
\hline $\mathrm{H}$ & 0.77323 & 0.70291 & 0.53595 \\
\hline $\mathrm{H}$ & 0.80122 & 0.67298 & 0.54915 \\
\hline $\mathrm{H}$ & 0.23257 & 0.02784 & 0.42156 \\
\hline $\mathrm{H}$ & 0.23358 & 0.97914 & 0.42437 \\
\hline $\mathrm{H}$ & 0.26976 & 0.05899 & 0.39936 \\
\hline $\mathrm{H}$ & 0.27031 & 0.09116 & 0.43044 \\
\hline $\mathrm{H}$ & 0.19832 & 0.10616 & 0.4128 \\
\hline $\mathrm{H}$ & 0.19771 & 0.07371 & 0.3827 \\
\hline $\mathrm{H}$ & 0.20072 & 0.12519 & 0.44921 \\
\hline $\mathrm{H}$ & 0.18599 & 0.15623 & 0.47747 \\
\hline $\mathrm{H}$ & 0.19226 & 0.19022 & 0.50483 \\
\hline $\mathrm{H}$ & 0.26663 & 0.18344 & 0.49412 \\
\hline $\mathrm{H}$ & 0.2603 & 0.14973 & 0.46681 \\
\hline $\mathrm{H}$ & 0.20688 & 0.20272 & 0.54492 \\
\hline $\mathrm{H}$ & 0.21523 & 0.23628 & 0.57238 \\
\hline $\mathrm{H}$ & 0.27063 & 0.25727 & 0.52197 \\
\hline $\mathrm{H}$ & 0.26183 & 0.22444 & 0.49365 \\
\hline $\mathrm{H}$ & 0.21686 & 0.96946 & 0.23337 \\
\hline $\mathrm{H}$ & 0.21728 & 0.01822 & 0.23051 \\
\hline $\mathrm{H}$ & 0.26062 & 0.94016 & 0.25058 \\
\hline $\mathrm{H}$ & 0.25993 & 0.90818 & 0.21929 \\
\hline $\mathrm{H}$ & 0.18982 & 0.89101 & 0.24267 \\
\hline $\mathrm{H}$ & 0.19072 & 0.92275 & 0.27383 \\
\hline $\mathrm{H}$ & 0.19882 & 0.85771 & 0.22391 \\
\hline $\mathrm{H}$ & 0.19081 & 0.81845 & 0.20662 \\
\hline $\mathrm{H}$ & 0.19872 & 0.78693 & 0.17633 \\
\hline $\mathrm{H}$ & 0.25005 & 0.83003 & 0.13873 \\
\hline $\mathrm{H}$ & 0.24257 & 0.86116 & 0.16926 \\
\hline $\mathrm{H}$ & 0.19484 & 0.77599 & 0.13186 \\
\hline $\mathrm{H}$ & 0.20442 & 0.74384 & 0.10294 \\
\hline $\mathrm{H}$ & 0.27748 & 0.76028 & 0.1098 \\
\hline $\mathrm{H}$ & 0.2681 & 0.7922 & 0.13893 \\
\hline $\mathrm{H}$ & 0.58468 & 0.26234 & -0.14347 \\
\hline $\mathrm{H}$ & 0.58705 & 0.26607 & 0.80685 \\
\hline $\mathrm{H}$ & 0.55126 & 0.29874 & -0.09198 \\
\hline $\mathrm{H}$ & 0.58137 & 0.29671 & -0.05853 \\
\hline $\mathrm{H}$ & 0.5937 & 0.22455 & -0.07733 \\
\hline $\mathrm{H}$ & 0.56347 & 0.22654 & -0.11038 \\
\hline $\mathrm{H}$ & 0.62998 & 0.23631 & -0.0636 \\
\hline $\mathrm{H}$ & 0.67076 & 0.23132 & -0.04854 \\
\hline $\mathrm{H}$ & 0.70071 & 0.23563 & -0.01575 \\
\hline $\mathrm{H}$ & 0.65128 & 0.27112 & 0.03284 \\
\hline
\end{tabular}




\begin{tabular}{|r|r|r|r|}
\hline $\mathrm{H}$ & 0.62178 & 0.26774 & -0.00032 \\
\hline $\mathrm{H}$ & 0.71377 & 0.22362 & 0.02387 \\
\hline $\mathrm{H}$ & 0.74435 & 0.2293 & 0.05572 \\
\hline $\mathrm{H}$ & 0.71535 & 0.29625 & 0.07524 \\
\hline $\mathrm{H}$ & 0.68487 & 0.29094 & 0.04337 \\
\hline $\mathrm{H}$ & 0.41212 & 0.26347 & 0.79606 \\
\hline $\mathrm{H}$ & 0.40957 & 0.26039 & -0.15408 \\
\hline $\mathrm{H}$ & 0.42909 & 0.30647 & 0.7656 \\
\hline $\mathrm{H}$ & 0.4013 & 0.30765 & 0.72976 \\
\hline $\mathrm{H}$ & 0.42206 & 0.23803 & 0.70818 \\
\hline $\mathrm{H}$ & 0.44971 & 0.23711 & 0.74362 \\
\hline $\mathrm{H}$ & 0.40619 & 0.24898 & 0.67165 \\
\hline $\mathrm{H}$ & 0.38611 & 0.23632 & 0.63242 \\
\hline $\mathrm{H}$ & 0.35334 & 0.23844 & 0.6025 \\
\hline $\mathrm{H}$ & 0.32364 & 0.29471 & 0.6466 \\
\hline $\mathrm{H}$ & 0.35641 & 0.29287 & 0.67607 \\
\hline $\mathrm{H}$ & 0.31189 & 0.22922 & 0.60412 \\
\hline $\mathrm{H}$ & 0.27525 & 0.22938 & 0.58047 \\
\hline $\mathrm{H}$ & 0.27595 & 0.30458 & 0.57953 \\
\hline $\mathrm{H}$ & 0.31276 & 0.30448 & 0.60311 \\
\hline & & &
\end{tabular}

Table S6. Fractional atomic coordinates for the unit cell of COF-1 with the interpenetrated ffc topology.

\begin{tabular}{|c|r|r|r|}
\hline \multicolumn{3}{|l|}{ Space group: $14 / \mathrm{m}$} & \multicolumn{1}{l|}{} \\
\hline $\mathrm{a}=\mathrm{b}=66.5298 \AA, \mathrm{c}=32.3478 \AA$ & \multicolumn{1}{l|}{$\mathrm{y}$} \\
\hline Atom & \multicolumn{1}{|c|}{$\mathrm{x}$} & \multicolumn{1}{l|}{$\mathrm{z}$} \\
\hline $\mathrm{C}$ & 0.91336 & 0.99609 & 0.53903 \\
\hline $\mathrm{C}$ & 0.89179 & 0.99519 & 0.539 \\
\hline $\mathrm{C}$ & 0.8144 & 0.00833 & 0.46124 \\
\hline $\mathrm{C}$ & 0.55358 & 0.99857 & 0.11187 \\
\hline $\mathrm{C}$ & 0.56254 & 0.01691 & 0.12683 \\
\hline $\mathrm{C}$ & 0.57704 & 0.01634 & 0.16013 \\
\hline $\mathrm{C}$ & 0.58264 & 0.99754 & 0.17917 \\
\hline $\mathrm{C}$ & 0.57399 & 0.97907 & 0.16324 \\
\hline $\mathrm{C}$ & 0.5595 & 0.97965 & 0.12991 \\
\hline $\mathrm{N}$ & 0.59707 & 0.99796 & 0.21436 \\
\hline $\mathrm{C}$ & 0.60051 & 0.98307 & 0.24149 \\
\hline $\mathrm{C}$ & 0.61532 & 0.9857 & 0.27721 \\
\hline $\mathrm{C}$ & 0.61741 & 0.96994 & 0.30785 \\
\hline
\end{tabular}




\begin{tabular}{|c|c|c|c|}
\hline $\mathrm{C}$ & 0.63072 & 0.97233 & 0.34287 \\
\hline $\mathrm{C}$ & 0.6423 & 0.99055 & 0.34792 \\
\hline $\mathrm{C}$ & 0.64044 & 0.0062 & 0.31679 \\
\hline $\mathrm{C}$ & 0.62708 & 0.00382 & 0.28179 \\
\hline $\mathrm{C}$ & 0.65581 & 0.99339 & 0.38613 \\
\hline $\mathrm{C}$ & 0.66738 & 0.97677 & 0.40262 \\
\hline $\mathrm{C}$ & 0.67934 & 0.97924 & 0.4397 \\
\hline $\mathrm{C}$ & 0.68 & 0.99835 & 0.46075 \\
\hline $\mathrm{C}$ & 0.66879 & 0.01505 & 0.4442 \\
\hline $\mathrm{C}$ & 0.65678 & 0.0126 & 0.4072 \\
\hline $\mathrm{C}$ & 0.79285 & 0.00701 & 0.53889 \\
\hline $\mathrm{C}$ & 0.74653 & 0.00433 & 0.53878 \\
\hline $\mathrm{C}$ & 0.725 & 0.00295 & 0.53872 \\
\hline $\mathrm{C}$ & 0.00036 & 0.51664 & 0.90881 \\
\hline $\mathrm{C}$ & 0.00083 & 0.54387 & 0.03467 \\
\hline $\mathrm{C}$ & 0.00136 & 0.56437 & 0.02195 \\
\hline $\mathrm{C}$ & 0.51052 & 0.99978 & 0.86609 \\
\hline $\mathrm{C}$ & 0.46254 & 0.0008 & 0.07797 \\
\hline $\mathrm{H}$ & 0.92212 & 0.99644 & 0.57084 \\
\hline $\mathrm{H}$ & 0.88298 & 0.99474 & 0.57075 \\
\hline $\mathrm{H}$ & 0.82323 & 0.00888 & 0.42953 \\
\hline $\mathrm{H}$ & 0.55801 & 0.03233 & 0.11185 \\
\hline $\mathrm{H}$ & 0.58443 & 0.03127 & 0.17203 \\
\hline $\mathrm{H}$ & 0.5788 & 0.96359 & 0.17754 \\
\hline $\mathrm{H}$ & 0.55251 & 0.96465 & 0.11743 \\
\hline $\mathrm{H}$ & 0.59205 & 0.96773 & 0.23789 \\
\hline $\mathrm{H}$ & 0.6082 & 0.95503 & 0.3043 \\
\hline $\mathrm{H}$ & 0.63227 & 0.95945 & 0.36767 \\
\hline $\mathrm{H}$ & 0.64987 & 0.02099 & 0.31996 \\
\hline $\mathrm{H}$ & 0.62575 & 0.01668 & 0.25689 \\
\hline $\mathrm{H}$ & 0.66705 & 0.96115 & 0.38571 \\
\hline $\mathrm{H}$ & 0.68859 & 0.96568 & 0.45292 \\
\hline $\mathrm{H}$ & 0.66944 & 0.03071 & 0.46092 \\
\hline $\mathrm{H}$ & 0.64772 & 0.02633 & 0.3941 \\
\hline $\mathrm{H}$ & 0.78448 & 0.00646 & 0.57115 \\
\hline $\mathrm{H}$ & 0.75529 & 0.00496 & 0.57057 \\
\hline $\mathrm{H}$ & 0.71647 & 0.00257 & 0.57081 \\
\hline $\mathrm{H}$ & 0.0017 & 0.57715 & 0.04716 \\
\hline $\mathrm{H}$ & 0.52261 & 0.99954 & 0.83943 \\
\hline $\mathrm{C}$ & 0.99761 & 0.0529 & 0.5 \\
\hline $\mathrm{C}$ & 0.97887 & 0.04184 & 0.5 \\
\hline $\mathrm{C}$ & 0.95961 & 0.05116 & 0.5 \\
\hline
\end{tabular}




\begin{tabular}{|r|r|r|r|}
\hline $\mathrm{C}$ & 0.92422 & 0.99658 & 0.5 \\
\hline $\mathrm{C}$ & 0.88095 & 0.99483 & 0.5 \\
\hline $\mathrm{N}$ & 0.85891 & 0.99393 & 0.5 \\
\hline $\mathrm{C}$ & 0.78139 & 0.00635 & 0.5 \\
\hline $\mathrm{C}$ & 0.75798 & 0.00497 & 0.5 \\
\hline $\mathrm{C}$ & 0.71386 & 0.00204 & 0.5 \\
\hline $\mathrm{C}$ & 0.95642 & 0.01727 & 0.5 \\
\hline $\mathrm{C}$ & 0.9454 & 0.03561 & 0.5 \\
\hline $\mathrm{N}$ & 0.97702 & 0.02099 & 0.5 \\
\hline $\mathrm{N}$ & 0.69148 & 0.00055 & 0.5 \\
\hline $\mathrm{C}$ & 0.84776 & 0.01054 & 0.5 \\
\hline $\mathrm{C}$ & 0.82521 & 0.00898 & 0.5 \\
\hline $\mathrm{H}$ & 0.95832 & 0.06872 & 0.5 \\
\hline $\mathrm{H}$ & 0.9278 & 0.03531 & 0.5 \\
\hline $\mathrm{H}$ & 0.85549 & 0.02636 & 0.5 \\
\hline $\mathrm{N}$ & 0.46883 & 0.00049 & 0 \\
\hline $\mathrm{N}$ & & 0.5 & 0.06473 \\
\hline
\end{tabular}

Table S7. Fractional atomic coordinates for the unit cell of COF-1 with the interpenetrated fjh topology.

\begin{tabular}{|c|c|c|c|}
\hline \multicolumn{4}{|c|}{ Space group: $P$ cca } \\
\hline \multicolumn{4}{|c|}{$\begin{array}{l}\mathrm{a}=81.7704 \AA, \mathrm{b}=81.7325 \AA, \mathrm{c}=53.2629 \AA \\
\alpha=\beta=\gamma=90^{\circ}\end{array}$} \\
\hline Atom & $\mathrm{x}$ & $\mathrm{y}$ & $\mathrm{z}$ \\
\hline $\mathrm{C}$ & 0.36442 & -0.1347 & 0.57167 \\
\hline $\mathrm{C}$ & 0.37988 & -0.12041 & 0.54582 \\
\hline $\mathrm{C}$ & 0.39853 & -0.09931 & 0.64299 \\
\hline $\mathrm{C}$ & 0.40935 & -0.08739 & 0.63486 \\
\hline $\mathrm{C}$ & 0.39433 & -0.10389 & 0.57749 \\
\hline $\mathrm{C}$ & 0.40354 & -0.09537 & 0.55738 \\
\hline $\mathrm{C}$ & 0.39687 & -0.08164 & 0.54541 \\
\hline $\mathrm{C}$ & 0.40597 & -0.07318 & 0.52737 \\
\hline $\mathrm{C}$ & 0.42189 & -0.07818 & 0.52133 \\
\hline $\mathrm{C}$ & 0.42835 & -0.09227 & 0.5329 \\
\hline $\mathrm{C}$ & 0.41923 & -0.10075 & 0.55085 \\
\hline $\mathrm{N}$ & 0.431 & -0.06881 & 0.50326 \\
\hline $\mathrm{C}$ & 0.44684 & -0.06865 & 0.50125 \\
\hline $\mathrm{C}$ & 0.45509 & -0.05867 & 0.48192 \\
\hline $\mathrm{C}$ & 0.47223 & -0.05848 & 0.48097 \\
\hline
\end{tabular}




\begin{tabular}{|c|c|c|c|}
\hline $\mathrm{C}$ & 0.48039 & -0.04917 & 0.46291 \\
\hline $\mathrm{C}$ & 0.47157 & -0.03978 & 0.44541 \\
\hline $\mathrm{C}$ & 0.45438 & -0.04001 & 0.44636 \\
\hline $\mathrm{C}$ & 0.44621 & -0.04935 & 0.46444 \\
\hline $\mathrm{C}$ & 0.48032 & -0.02981 & 0.42631 \\
\hline $\mathrm{C}$ & 0.49476 & -0.02143 & 0.43265 \\
\hline $\mathrm{C}$ & 0.50316 & -0.01229 & 0.41465 \\
\hline $\mathrm{C}$ & 0.49735 & -0.01134 & 0.3899 \\
\hline $\mathrm{C}$ & 0.48286 & -0.01959 & 0.38354 \\
\hline $\mathrm{C}$ & 0.47442 & -0.02872 & 0.40156 \\
\hline $\mathrm{C}$ & 0.86331 & 0.3703 & 1.08252 \\
\hline $\mathrm{C}$ & 0.87821 & 0.38632 & 1.05827 \\
\hline $\mathrm{C}$ & 0.89582 & 0.40407 & 0.1575 \\
\hline $\mathrm{C}$ & 0.90621 & 0.41669 & 0.15064 \\
\hline $\mathrm{C}$ & 0.89174 & 0.40222 & 1.09165 \\
\hline $\mathrm{C}$ & 0.90086 & 0.41184 & 1.07262 \\
\hline $\mathrm{C}$ & 0.89412 & 0.42615 & 1.06243 \\
\hline $\mathrm{C}$ & 0.90339 & 0.4359 & 1.0462 \\
\hline $\mathrm{C}$ & 0.91959 & 0.43174 & 1.04053 \\
\hline $\mathrm{C}$ & 0.92612 & 0.41709 & 1.05015 \\
\hline $\mathrm{C}$ & 0.9168 & 0.40724 & 1.06608 \\
\hline $\mathrm{N}$ & 0.92895 & 0.4424 & 1.02458 \\
\hline $\mathrm{C}$ & 0.9448 & 0.44363 & 1.02492 \\
\hline $\mathrm{C}$ & 0.95351 & 0.4544 & 1.00712 \\
\hline $\mathrm{C}$ & 0.9705 & 0.45628 & 1.00928 \\
\hline $\mathrm{C}$ & 0.97914 & 0.46587 & 0.99207 \\
\hline $\mathrm{C}$ & 0.97095 & 0.47376 & 0.97227 \\
\hline $\mathrm{C}$ & 0.95387 & 0.47204 & 0.97037 \\
\hline $\mathrm{C}$ & 0.94523 & 0.46249 & 0.98763 \\
\hline $\mathrm{C}$ & 0.98018 & 0.4836 & 0.95354 \\
\hline $\mathrm{C}$ & 0.99337 & 0.49349 & 0.96107 \\
\hline $\mathrm{C}$ & 1.00205 & 0.50275 & 0.94351 \\
\hline $\mathrm{C}$ & 0.99779 & 0.50226 & 0.91798 \\
\hline $\mathrm{C}$ & 0.98472 & 0.49229 & 0.91036 \\
\hline $\mathrm{C}$ & 0.97598 & 0.48308 & 0.92795 \\
\hline $\mathrm{N}$ & 0.39137 & -0.10688 & 0.6232 \\
\hline $\mathrm{C}$ & 0.79177 & -0.03028 & 0.87924 \\
\hline $\mathrm{C}$ & 0.78375 & -0.01535 & 0.879 \\
\hline $\mathrm{C}$ & 0.74985 & -0.04204 & 0.88007 \\
\hline $\mathrm{C}$ & 0.73467 & -0.03388 & 0.87965 \\
\hline $\mathrm{C}$ & 0.708 & -0.03 & 0.87893 \\
\hline $\mathrm{C}$ & 0.68997 & 0.00022 & 0.87797 \\
\hline
\end{tabular}




\begin{tabular}{|c|c|c|c|}
\hline $\mathrm{C}$ & 0.68099 & -0.00316 & 0.89979 \\
\hline $\mathrm{C}$ & 0.66392 & -0.00311 & 0.89915 \\
\hline $\mathrm{C}$ & 0.65559 & 0.00066 & 0.87692 \\
\hline $\mathrm{C}$ & 0.66462 & 0.00388 & 0.85496 \\
\hline $\mathrm{C}$ & 0.6817 & 0.00363 & 0.85552 \\
\hline $\mathrm{N}$ & 0.63804 & 0.00056 & 0.87716 \\
\hline $\mathrm{C}$ & 0.62872 & 0.00866 & 0.86181 \\
\hline $\mathrm{C}$ & 0.61076 & 0.0075 & 0.86361 \\
\hline $\mathrm{C}$ & 0.60099 & 0.01821 & 0.84955 \\
\hline $\mathrm{C}$ & 0.58396 & 0.01738 & 0.85107 \\
\hline $\mathrm{C}$ & 0.57637 & 0.00572 & 0.86658 \\
\hline $\mathrm{C}$ & 0.58617 & -0.00511 & 0.88048 \\
\hline $\mathrm{C}$ & 0.60321 & -0.0042 & 0.87909 \\
\hline $\mathrm{C}$ & 0.5583 & 0.00483 & 0.86819 \\
\hline $\mathrm{C}$ & 0.5489 & 0.0192 & 0.86962 \\
\hline $\mathrm{C}$ & 0.53186 & 0.01835 & 0.87097 \\
\hline $\mathrm{C}$ & 0.52394 & 0.00314 & 0.87068 \\
\hline $\mathrm{C}$ & 0.53328 & -0.01122 & 0.86931 \\
\hline $\mathrm{C}$ & 0.55031 & -0.0104 & 0.8682 \\
\hline $\mathrm{N}$ & 0.50635 & -0.00227 & 0.3712 \\
\hline $\mathrm{C}$ & 0.38293 & 0.11596 & 1.07047 \\
\hline $\mathrm{C}$ & 0.36818 & 0.13229 & 1.04658 \\
\hline $\mathrm{C}$ & 0.34945 & 0.14817 & 0.1461 \\
\hline $\mathrm{C}$ & 0.33736 & 0.15921 & 0.13936 \\
\hline $\mathrm{C}$ & 0.35267 & 0.14601 & 1.08018 \\
\hline $\mathrm{C}$ & 0.34372 & 0.15581 & 1.06121 \\
\hline $\mathrm{C}$ & 0.3301 & 0.14924 & 1.04884 \\
\hline $\mathrm{C}$ & 0.32145 & 0.1587 & 1.03149 \\
\hline $\mathrm{C}$ & 0.32613 & 0.17488 & 1.02656 \\
\hline $\mathrm{C}$ & 0.34001 & 0.18134 & 1.03872 \\
\hline $\mathrm{C}$ & 0.34869 & 0.17184 & 1.05596 \\
\hline $\mathrm{N}$ & 0.31685 & 0.18412 & 1.0086 \\
\hline $\mathrm{C}$ & 0.31654 & 0.19997 & 1.00677 \\
\hline $\mathrm{C}$ & 0.30689 & 0.20808 & 0.98694 \\
\hline $\mathrm{C}$ & 0.30682 & 0.22521 & 0.98538 \\
\hline $\mathrm{C}$ & 0.29807 & 0.23312 & 0.9664 \\
\hline $\mathrm{C}$ & 0.28917 & 0.22404 & 0.94862 \\
\hline $\mathrm{C}$ & 0.28915 & 0.20688 & 0.95037 \\
\hline $\mathrm{C}$ & 0.29791 & 0.19897 & 0.96932 \\
\hline $\mathrm{C}$ & 0.27999 & 0.23241 & 0.92828 \\
\hline $\mathrm{C}$ & 0.27145 & 0.247 & 0.93312 \\
\hline $\mathrm{C}$ & 0.2628 & 0.25485 & 0.914 \\
\hline
\end{tabular}




\begin{tabular}{|c|c|c|c|}
\hline $\mathrm{C}$ & 0.26254 & 0.24832 & 0.88962 \\
\hline $\mathrm{C}$ & 0.27105 & 0.23376 & 0.88471 \\
\hline $\mathrm{C}$ & 0.27968 & 0.22587 & 0.90384 \\
\hline $\mathrm{C}$ & 0.88091 & -0.38995 & 0.58333 \\
\hline $\mathrm{C}$ & 0.86711 & -0.37385 & 0.55776 \\
\hline $\mathrm{C}$ & 0.84776 & -0.35417 & 0.65549 \\
\hline $\mathrm{C}$ & 0.83602 & -0.34309 & 0.64759 \\
\hline $\mathrm{C}$ & 0.85201 & -0.35818 & 0.58983 \\
\hline $\mathrm{C}$ & 0.84377 & -0.34847 & 0.56994 \\
\hline $\mathrm{C}$ & 0.83049 & -0.35501 & 0.55666 \\
\hline $\mathrm{C}$ & 0.82212 & -0.34543 & 0.53911 \\
\hline $\mathrm{C}$ & 0.82673 & -0.32914 & 0.53485 \\
\hline $\mathrm{C}$ & 0.84036 & -0.32275 & 0.54772 \\
\hline $\mathrm{C}$ & 0.84876 & -0.33236 & 0.56518 \\
\hline $\mathrm{N}$ & 0.81748 & -0.31962 & 0.51713 \\
\hline $\mathrm{C}$ & 0.81698 & -0.30373 & 0.5162 \\
\hline $\mathrm{C}$ & 0.80713 & -0.29519 & 0.497 \\
\hline $\mathrm{C}$ & 0.80607 & -0.27806 & 0.49751 \\
\hline $\mathrm{C}$ & 0.79686 & -0.26969 & 0.47952 \\
\hline $\mathrm{C}$ & 0.78853 & -0.27831 & 0.46059 \\
\hline $\mathrm{C}$ & 0.7896 & -0.29548 & 0.46012 \\
\hline $\mathrm{C}$ & 0.79878 & -0.30384 & 0.47815 \\
\hline $\mathrm{C}$ & 0.77866 & -0.26944 & 0.44151 \\
\hline $\mathrm{C}$ & 0.769 & -0.25596 & 0.44847 \\
\hline $\mathrm{C}$ & 0.75957 & -0.24773 & 0.43061 \\
\hline $\mathrm{C}$ & 0.75961 & -0.25278 & 0.4054 \\
\hline $\mathrm{C}$ & 0.76934 & -0.26617 & 0.39836 \\
\hline $\mathrm{C}$ & 0.77875 & -0.27443 & 0.41623 \\
\hline $\mathrm{N}$ & 0.35663 & 0.14166 & 1.12545 \\
\hline $\mathrm{C}$ & 0.27919 & -0.45753 & 0.37965 \\
\hline $\mathrm{C}$ & 0.26447 & -0.46592 & 0.37936 \\
\hline $\mathrm{C}$ & 0.29198 & -0.49911 & 0.38274 \\
\hline $\mathrm{C}$ & 0.28425 & 0.48549 & 0.38174 \\
\hline $\mathrm{C}$ & 0.28105 & 0.45873 & 0.38011 \\
\hline $\mathrm{C}$ & 1.25115 & 0.44 & 0.37712 \\
\hline $\mathrm{C}$ & 0.25457 & 0.43072 & 0.39862 \\
\hline $\mathrm{C}$ & 0.25405 & 0.41365 & 0.39761 \\
\hline $\mathrm{C}$ & 1.24972 & 0.40565 & 0.37534 \\
\hline $\mathrm{C}$ & 1.24652 & 0.41496 & 0.35365 \\
\hline $\mathrm{C}$ & 1.24729 & 0.43203 & 0.35457 \\
\hline $\mathrm{N}$ & 1.24922 & 0.3881 & 0.37526 \\
\hline $\mathrm{C}$ & 1.24045 & 0.37914 & 0.36029 \\
\hline
\end{tabular}




\begin{tabular}{|c|c|c|c|}
\hline $\mathrm{C}$ & 1.2412 & 0.36113 & 0.36178 \\
\hline $\mathrm{C}$ & 1.22985 & 0.35164 & 0.34844 \\
\hline $\mathrm{C}$ & 1.23048 & 0.33456 & 0.34956 \\
\hline $\mathrm{C}$ & 1.24257 & 0.32667 & 0.36392 \\
\hline $\mathrm{C}$ & 0.25392 & 0.33622 & 0.37725 \\
\hline $\mathrm{C}$ & 0.25322 & 0.35327 & 0.37626 \\
\hline $\mathrm{C}$ & 1.24343 & 0.30857 & 0.36501 \\
\hline $\mathrm{C}$ & 1.22911 & 0.29917 & 0.36729 \\
\hline $\mathrm{C}$ & 1.23001 & 0.28213 & 0.36886 \\
\hline $\mathrm{C}$ & 1.24522 & 0.27419 & 0.36798 \\
\hline $\mathrm{C}$ & 0.2595 & 0.28353 & 0.36548 \\
\hline $\mathrm{C}$ & 0.25863 & 0.30056 & 0.36408 \\
\hline $\mathrm{N}$ & 0.25383 & 0.25663 & 0.86982 \\
\hline $\mathrm{C}$ & 0.6365 & 0.36825 & 0.68912 \\
\hline $\mathrm{C}$ & 0.62031 & 0.3821 & 0.71449 \\
\hline $\mathrm{C}$ & 0.60497 & 0.40551 & 0.61713 \\
\hline $\mathrm{C}$ & 0.5943 & 0.41762 & 0.62512 \\
\hline $\mathrm{C}$ & 0.60667 & 0.3991 & 0.68254 \\
\hline $\mathrm{C}$ & 0.59664 & 0.40708 & 0.70228 \\
\hline $\mathrm{C}$ & 0.60309 & 0.4199 & 0.71666 \\
\hline $\mathrm{C}$ & 0.59335 & 0.42775 & 0.73457 \\
\hline $\mathrm{C}$ & 0.57702 & 0.42307 & 0.73811 \\
\hline $\mathrm{C}$ & 0.57068 & 0.40999 & 0.7239 \\
\hline $\mathrm{C}$ & 0.58043 & 0.4021 & 0.70609 \\
\hline $\mathrm{N}$ & 0.56751 & 0.43151 & 0.75675 \\
\hline $\mathrm{C}$ & 0.55163 & 0.43185 & 0.75799 \\
\hline $\mathrm{C}$ & 0.5432 & 0.44051 & 0.77861 \\
\hline $\mathrm{C}$ & 0.52605 & 0.44049 & 0.77957 \\
\hline $\mathrm{C}$ & 0.51781 & 0.44826 & 0.79924 \\
\hline $\mathrm{C}$ & 0.52657 & 0.45629 & 0.81834 \\
\hline $\mathrm{C}$ & 0.54375 & 0.45644 & 0.81717 \\
\hline $\mathrm{C}$ & 0.55199 & 0.44863 & 0.79754 \\
\hline $\mathrm{C}$ & 0.51784 & 0.46445 & 0.83943 \\
\hline $\mathrm{C}$ & 0.50314 & 0.47295 & 0.83519 \\
\hline $\mathrm{C}$ & 0.49504 & 0.48072 & 0.85497 \\
\hline $\mathrm{C}$ & 0.50142 & 0.48014 & 0.87943 \\
\hline $\mathrm{C}$ & 0.51604 & 0.4716 & 0.88375 \\
\hline $\mathrm{C}$ & 0.52417 & 0.46383 & 0.86398 \\
\hline $\mathrm{C}$ & 1.13433 & -0.13318 & 0.1782 \\
\hline $\mathrm{C}$ & 1.11811 & -0.11833 & 0.2021 \\
\hline $\mathrm{C}$ & 1.10214 & -0.09969 & 1.10256 \\
\hline $\mathrm{C}$ & 1.09106 & -0.08763 & 1.10929 \\
\hline
\end{tabular}




\begin{tabular}{|c|c|c|c|}
\hline $\mathrm{C}$ & 1.10428 & -0.10294 & 0.16848 \\
\hline $\mathrm{C}$ & 1.09455 & -0.09395 & 0.18747 \\
\hline $\mathrm{C}$ & 1.10143 & -0.08078 & 0.20058 \\
\hline $\mathrm{C}$ & 1.09204 & -0.07204 & 0.2179 \\
\hline $\mathrm{C}$ & 1.07564 & -0.07619 & 0.22209 \\
\hline $\mathrm{C}$ & 1.06888 & -0.08967 & 0.20929 \\
\hline $\mathrm{C}$ & 1.07829 & -0.09845 & 0.19206 \\
\hline $\mathrm{N}$ & 1.06642 & -0.06669 & 0.2398 \\
\hline $\mathrm{C}$ & 1.05057 & -0.06586 & 0.24094 \\
\hline $\mathrm{C}$ & 1.04245 & -0.05581 & 0.26025 \\
\hline $\mathrm{C}$ & 1.02535 & -0.05447 & 0.26023 \\
\hline $\mathrm{C}$ & 1.0174 & -0.04505 & 0.27837 \\
\hline $\mathrm{C}$ & 1.02642 & -0.0368 & 0.29694 \\
\hline $\mathrm{C}$ & 1.04355 & -0.03816 & 0.29693 \\
\hline $\mathrm{C}$ & 1.0515 & -0.04755 & 0.27875 \\
\hline $\mathrm{C}$ & 1.018 & -0.02672 & 0.31616 \\
\hline $\mathrm{C}$ & 1.00465 & -0.01682 & 0.30946 \\
\hline $\mathrm{C}$ & 0.99677 & -0.00725 & 0.32748 \\
\hline $\mathrm{C}$ & 1.00204 & -0.00742 & 0.35257 \\
\hline $\mathrm{C}$ & 1.01533 & -0.01734 & 0.35934 \\
\hline $\mathrm{C}$ & 1.02326 & -0.02689 & 0.3413 \\
\hline $\mathrm{N}$ & 0.61116 & 0.39719 & 0.63697 \\
\hline $\mathrm{C}$ & 1.20878 & 0.46901 & 0.38099 \\
\hline $\mathrm{C}$ & 1.21648 & 0.4841 & 0.38158 \\
\hline $\mathrm{C}$ & 0.25092 & 0.45814 & 0.37852 \\
\hline $\mathrm{C}$ & 0.26599 & 0.46649 & 0.37964 \\
\hline $\mathrm{C}$ & 0.29259 & 0.47074 & 0.3817 \\
\hline $\mathrm{C}$ & 0.31012 & -0.49855 & 0.38462 \\
\hline $\mathrm{C}$ & 0.31983 & 0.49796 & 0.36359 \\
\hline $\mathrm{C}$ & 0.33685 & 0.49916 & 0.36511 \\
\hline $\mathrm{C}$ & 0.34438 & -0.49575 & 0.38739 \\
\hline $\mathrm{C}$ & 0.33465 & -0.49244 & 0.40859 \\
\hline $\mathrm{C}$ & 0.31764 & -0.49389 & 0.40719 \\
\hline $\mathrm{N}$ & 0.3619 & -0.49468 & 0.38809 \\
\hline $\mathrm{C}$ & 0.3703 & -0.48513 & 0.40273 \\
\hline $\mathrm{C}$ & 0.38835 & -0.4854 & 0.40228 \\
\hline $\mathrm{C}$ & 0.39717 & -0.4733 & 0.41512 \\
\hline $\mathrm{C}$ & 0.41428 & -0.47353 & 0.4153 \\
\hline $\mathrm{C}$ & 0.42289 & -0.48595 & 0.40269 \\
\hline $\mathrm{C}$ & 0.414 & 0.50198 & 0.38974 \\
\hline $\mathrm{C}$ & 0.39693 & 0.50226 & 0.38948 \\
\hline $\mathrm{C}$ & 0.44104 & -0.4864 & 0.40292 \\
\hline
\end{tabular}




\begin{tabular}{|c|c|c|c|}
\hline $\mathrm{C}$ & 0.45022 & -0.47195 & 0.40026 \\
\hline $\mathrm{C}$ & 0.4673 & -0.47256 & 0.39939 \\
\hline $\mathrm{C}$ & 0.47551 & -0.48758 & 0.40141 \\
\hline $\mathrm{C}$ & 0.4664 & 0.49803 & 0.40455 \\
\hline $\mathrm{C}$ & 0.44935 & 0.4986 & 0.40524 \\
\hline $\mathrm{N}$ & 0.4931 & 0.48829 & 0.89982 \\
\hline $\mathrm{C}$ & 0.61786 & -0.3869 & 0.18983 \\
\hline $\mathrm{C}$ & 0.63214 & -0.37033 & 0.21405 \\
\hline $\mathrm{C}$ & 0.65195 & -0.3546 & 1.11502 \\
\hline $\mathrm{C}$ & 0.66378 & -0.34334 & 1.12201 \\
\hline $\mathrm{C}$ & 0.64844 & -0.35703 & 0.18093 \\
\hline $\mathrm{C}$ & 0.65727 & -0.34729 & 0.20017 \\
\hline $\mathrm{C}$ & 0.67093 & -0.35383 & 0.21249 \\
\hline $\mathrm{C}$ & 0.67946 & -0.34446 & 0.23013 \\
\hline $\mathrm{C}$ & 0.67459 & -0.32838 & 0.23548 \\
\hline $\mathrm{C}$ & 0.66064 & -0.32198 & 0.22343 \\
\hline $\mathrm{C}$ & 0.6521 & -0.33138 & 0.20587 \\
\hline $\mathrm{N}$ & 0.68386 & -0.31916 & 0.25357 \\
\hline $\mathrm{C}$ & 0.68394 & -0.30331 & 0.25568 \\
\hline $\mathrm{C}$ & 0.6938 & -0.29506 & 0.27518 \\
\hline $\mathrm{C}$ & 0.69399 & -0.27791 & 0.27623 \\
\hline $\mathrm{C}$ & 0.70318 & -0.26976 & 0.29447 \\
\hline $\mathrm{C}$ & 0.71244 & -0.27861 & 0.3121 \\
\hline $\mathrm{C}$ & 0.71222 & -0.29581 & 0.31106 \\
\hline $\mathrm{C}$ & 0.70301 & -0.30396 & 0.29279 \\
\hline $\mathrm{C}$ & 0.7223 & -0.2699 & 0.33141 \\
\hline $\mathrm{C}$ & 0.73074 & -0.25544 & 0.3253 \\
\hline $\mathrm{C}$ & 0.73987 & -0.24714 & 0.34345 \\
\hline $\mathrm{C}$ & 0.74076 & -0.25307 & 0.36815 \\
\hline $\mathrm{C}$ & 0.7324 & -0.26754 & 0.37431 \\
\hline $\mathrm{C}$ & 0.72328 & -0.27587 & 0.35614 \\
\hline $\mathrm{C}$ & 1.1156 & 0.11467 & 0.677 \\
\hline $\mathrm{C}$ & 1.12998 & 0.13004 & 0.70286 \\
\hline $\mathrm{C}$ & 1.15098 & 0.14878 & 0.60567 \\
\hline $\mathrm{C}$ & 1.16291 & 0.1596 & 0.61379 \\
\hline $\mathrm{C}$ & 1.14636 & 0.14462 & 0.67117 \\
\hline $\mathrm{C}$ & 1.15494 & 0.15376 & 0.69127 \\
\hline $\mathrm{C}$ & 1.1681 & 0.14664 & 0.70412 \\
\hline $\mathrm{C}$ & 1.17677 & 0.1557 & 0.72197 \\
\hline $\mathrm{C}$ & 1.17256 & 0.17201 & 0.72693 \\
\hline $\mathrm{C}$ & 1.15896 & 0.17891 & 0.71461 \\
\hline $\mathrm{C}$ & 1.15028 & 0.16984 & 0.69685 \\
\hline
\end{tabular}




\begin{tabular}{|c|c|c|c|}
\hline $\mathrm{N}$ & 1.18228 & 0.18111 & 0.74455 \\
\hline $\mathrm{C}$ & 1.18329 & 0.19697 & 0.74542 \\
\hline $\mathrm{C}$ & 1.19369 & 0.20525 & 0.76414 \\
\hline $\mathrm{C}$ & 1.19528 & 0.22232 & 0.76325 \\
\hline $\mathrm{C}$ & 1.20513 & 0.23051 & 0.78058 \\
\hline $\mathrm{C}$ & 1.21362 & 0.22177 & 0.7992 \\
\hline $\mathrm{C}$ & 1.21196 & 0.20466 & 0.80016 \\
\hline $\mathrm{C}$ & 1.20211 & 0.19646 & 0.78277 \\
\hline $\mathrm{C}$ & 1.22422 & 0.23052 & 0.81742 \\
\hline $\mathrm{C}$ & 1.23405 & 0.24365 & 0.8095 \\
\hline $\mathrm{C}$ & 1.24385 & 0.25207 & 0.82663 \\
\hline $\mathrm{C}$ & 1.24401 & 0.24759 & 0.85207 \\
\hline $\mathrm{C}$ & 1.23426 & 0.23443 & 0.86003 \\
\hline $\mathrm{C}$ & 1.22448 & 0.22596 & 0.84287 \\
\hline $\mathrm{N}$ & 0.64484 & -0.36135 & 0.13554 \\
\hline $\mathrm{C}$ & 0.72003 & 0.04205 & 0.87965 \\
\hline $\mathrm{C}$ & 0.73492 & 0.03397 & 0.8798 \\
\hline $\mathrm{C}$ & 0.70812 & 0.00014 & 0.87862 \\
\hline $\mathrm{C}$ & 0.71613 & -0.01513 & 0.87897 \\
\hline $\mathrm{C}$ & 0.71972 & -0.04186 & 0.87954 \\
\hline $\mathrm{C}$ & 0.74979 & -0.06023 & 0.88064 \\
\hline $\mathrm{C}$ & 0.74629 & -0.06915 & 0.85881 \\
\hline $\mathrm{C}$ & 0.74617 & -0.08625 & 0.85938 \\
\hline $\mathrm{C}$ & 0.7499 & -0.09467 & 0.88156 \\
\hline $\mathrm{C}$ & 0.75328 & -0.08569 & 0.90353 \\
\hline $\mathrm{C}$ & 0.75319 & -0.06858 & 0.90303 \\
\hline $\mathrm{N}$ & 0.74958 & -0.11224 & 0.88127 \\
\hline $\mathrm{C}$ & 0.75756 & -0.12169 & 0.8966 \\
\hline $\mathrm{C}$ & 0.7562 & -0.13967 & 0.89482 \\
\hline $\mathrm{C}$ & 0.76681 & -0.14955 & 0.90892 \\
\hline $\mathrm{C}$ & 0.76579 & -0.16659 & 0.90748 \\
\hline $\mathrm{C}$ & 0.75405 & -0.1741 & 0.89204 \\
\hline $\mathrm{C}$ & 0.74332 & -0.16419 & 0.87811 \\
\hline $\mathrm{C}$ & 0.74442 & -0.14715 & 0.8794 \\
\hline $\mathrm{C}$ & 0.75302 & -0.1922 & 0.89046 \\
\hline $\mathrm{C}$ & 0.76732 & -0.20172 & 0.88901 \\
\hline $\mathrm{C}$ & 0.76635 & -0.21876 & 0.88762 \\
\hline $\mathrm{C}$ & 0.7511 & -0.22661 & 0.88789 \\
\hline $\mathrm{C}$ & 0.73682 & -0.21716 & 0.88935 \\
\hline $\mathrm{C}$ & 0.73775 & -0.20011 & 0.89049 \\
\hline $\mathrm{N}$ & 0.74987 & -0.24421 & 0.387 \\
\hline $\mathrm{N}$ & 0.37347 & -0.12472 & 0.58647 \\
\hline
\end{tabular}




\begin{tabular}{|c|c|c|c|}
\hline $\mathrm{N}$ & 0.62821 & -0.38015 & 1.09833 \\
\hline $\mathrm{N}$ & 0.62776 & 0.3784 & 0.67413 \\
\hline $\mathrm{N}$ & 0.37448 & 0.12379 & 0.16219 \\
\hline $\mathrm{N}$ & 0.76732 & -0.01753 & 0.87938 \\
\hline $\mathrm{N}$ & 0.26704 & -0.48229 & 0.38055 \\
\hline $\mathrm{N}$ & 0.73254 & -0.01743 & 0.87935 \\
\hline $\mathrm{N}$ & 0.26788 & 0.48295 & 0.38055 \\
\hline $\mathrm{H}$ & 0.38552 & -0.1156 & 0.52898 \\
\hline $\mathrm{H}$ & 0.41685 & -0.07933 & 0.64617 \\
\hline $\mathrm{H}$ & 0.38474 & -0.07738 & 0.5503 \\
\hline $\mathrm{H}$ & 0.40075 & -0.06249 & 0.51842 \\
\hline $\mathrm{H}$ & 0.44033 & -0.09689 & 0.52807 \\
\hline $\mathrm{H}$ & 0.42443 & -0.11144 & 0.55983 \\
\hline $\mathrm{H}$ & 0.45437 & -0.07547 & 0.51427 \\
\hline $\mathrm{H}$ & 0.47934 & -0.06558 & 0.49423 \\
\hline $\mathrm{H}$ & 0.49363 & -0.04947 & 0.46247 \\
\hline $\mathrm{H}$ & 0.44724 & -0.03278 & 0.43335 \\
\hline $\mathrm{H}$ & 0.43297 & -0.04922 & 0.46476 \\
\hline $\mathrm{H}$ & 0.49951 & -0.02181 & 0.45161 \\
\hline $\mathrm{H}$ & 0.5142 & -0.00589 & 0.42005 \\
\hline $\mathrm{H}$ & 0.47826 & -0.01921 & 0.36448 \\
\hline $\mathrm{H}$ & 0.46347 & -0.03521 & 0.39597 \\
\hline $\mathrm{H}$ & 0.88368 & 0.39207 & 1.04201 \\
\hline $\mathrm{H}$ & 0.91348 & 0.42444 & 0.16277 \\
\hline $\mathrm{H}$ & 0.88184 & 0.42989 & 1.06747 \\
\hline $\mathrm{H}$ & 0.89811 & 0.44699 & 1.03856 \\
\hline $\mathrm{H}$ & 0.93835 & 0.41316 & 1.04531 \\
\hline $\mathrm{H}$ & 0.9221 & 0.39616 & 1.07371 \\
\hline $\mathrm{H}$ & 0.95193 & 0.43688 & 1.03858 \\
\hline $\mathrm{H}$ & 0.97715 & 0.45019 & 1.02418 \\
\hline $\mathrm{H}$ & 0.99229 & 0.46681 & 0.99397 \\
\hline $\mathrm{H}$ & 0.94717 & 0.47818 & 0.95559 \\
\hline $\mathrm{H}$ & 0.93211 & 0.46131 & 0.98554 \\
\hline $\mathrm{H}$ & 0.99687 & 0.49421 & 0.98059 \\
\hline $\mathrm{H}$ & 1.01204 & 0.5104 & 0.94985 \\
\hline $\mathrm{H}$ & 0.98143 & 0.49146 & 0.89071 \\
\hline $\mathrm{H}$ & 0.96613 & 0.47532 & 0.92144 \\
\hline $\mathrm{H}$ & 0.80481 & -0.03251 & 0.87919 \\
\hline $\mathrm{H}$ & 0.69495 & -0.03215 & 0.87856 \\
\hline $\mathrm{H}$ & 0.68724 & -0.00586 & 0.91723 \\
\hline $\mathrm{H}$ & 0.65713 & -0.00582 & 0.91611 \\
\hline $\mathrm{H}$ & 0.65857 & 0.0063 & 0.83729 \\
\hline
\end{tabular}




\begin{tabular}{|c|c|c|c|}
\hline $\mathrm{H}$ & 0.6885 & 0.00607 & 0.83847 \\
\hline $\mathrm{H}$ & 0.63398 & 0.01687 & 0.84805 \\
\hline $\mathrm{H}$ & 0.60655 & 0.02729 & 0.83744 \\
\hline $\mathrm{H}$ & 0.5767 & 0.02572 & 0.83987 \\
\hline $\mathrm{H}$ & 0.58067 & -0.01416 & 0.89269 \\
\hline $\mathrm{H}$ & 0.61044 & -0.01268 & 0.89008 \\
\hline $\mathrm{H}$ & 0.55472 & 0.03109 & 0.8699 \\
\hline $\mathrm{H}$ & 0.52481 & 0.02954 & 0.87187 \\
\hline $\mathrm{H}$ & 0.52733 & -0.02306 & 0.86913 \\
\hline $\mathrm{H}$ & 0.55723 & -0.02166 & 0.86691 \\
\hline $\mathrm{H}$ & 0.36301 & 0.13845 & 1.03044 \\
\hline $\mathrm{H}$ & 0.32954 & 0.16631 & 0.15159 \\
\hline $\mathrm{H}$ & 0.32616 & 0.13685 & 1.05275 \\
\hline $\mathrm{H}$ & 0.31093 & 0.1535 & 1.02207 \\
\hline $\mathrm{H}$ & 0.34435 & 0.19356 & 1.03477 \\
\hline $\mathrm{H}$ & 0.35929 & 0.17698 & 1.06528 \\
\hline $\mathrm{H}$ & 0.32317 & 0.20754 & 1.01998 \\
\hline $\mathrm{H}$ & 0.31365 & 0.23249 & 0.99875 \\
\hline $\mathrm{H}$ & 0.29849 & 0.24635 & 0.96539 \\
\hline $\mathrm{H}$ & 0.28221 & 0.19955 & 0.93723 \\
\hline $\mathrm{H}$ & 0.29766 & 0.18573 & 0.97013 \\
\hline $\mathrm{H}$ & 0.27128 & 0.25224 & 0.95178 \\
\hline $\mathrm{H}$ & 0.25618 & 0.26598 & 0.91825 \\
\hline $\mathrm{H}$ & 0.2712 & 0.22864 & 0.86596 \\
\hline $\mathrm{H}$ & 0.28636 & 0.21481 & 0.89939 \\
\hline $\mathrm{H}$ & 0.86232 & -0.3681 & 0.541 \\
\hline $\mathrm{H}$ & 0.82816 & -0.33551 & 0.65907 \\
\hline $\mathrm{H}$ & 0.8265 & -0.36743 & 0.56019 \\
\hline $\mathrm{H}$ & 0.81179 & -0.35059 & 0.52915 \\
\hline $\mathrm{H}$ & 0.84467 & -0.31045 & 0.54431 \\
\hline $\mathrm{H}$ & 0.85906 & -0.32719 & 0.57521 \\
\hline $\mathrm{H}$ & 0.8234 & -0.29642 & 0.52998 \\
\hline $\mathrm{H}$ & 0.81241 & -0.27112 & 0.51185 \\
\hline $\mathrm{H}$ & 0.79642 & -0.25646 & 0.48024 \\
\hline $\mathrm{H}$ & 0.78313 & -0.30247 & 0.446 \\
\hline $\mathrm{H}$ & 0.79932 & -0.31707 & 0.47732 \\
\hline $\mathrm{H}$ & 0.76852 & -0.25192 & 0.46781 \\
\hline $\mathrm{H}$ & 0.75208 & -0.23752 & 0.43652 \\
\hline $\mathrm{H}$ & 0.76977 & -0.27009 & 0.37896 \\
\hline $\mathrm{H}$ & 0.78628 & -0.28459 & 0.4102 \\
\hline $\mathrm{H}$ & 0.28112 & -0.44444 & 0.37914 \\
\hline $\mathrm{H}$ & 0.28351 & 0.44573 & 0.37951 \\
\hline
\end{tabular}




\begin{tabular}{|c|c|c|c|}
\hline $\mathrm{H}$ & 0.25751 & 0.43672 & 0.41617 \\
\hline $\mathrm{H}$ & 0.25672 & 0.40662 & 0.41435 \\
\hline $\mathrm{H}$ & 1.24365 & 0.40911 & 0.33598 \\
\hline $\mathrm{H}$ & 1.24471 & 0.43907 & 0.33779 \\
\hline $\mathrm{H}$ & 1.23206 & 0.38474 & 0.3471 \\
\hline $\mathrm{H}$ & 1.22048 & 0.35744 & 0.33716 \\
\hline $\mathrm{H}$ & 1.22168 & 0.32751 & 0.3389 \\
\hline $\mathrm{H}$ & 0.26326 & 0.3305 & 0.38868 \\
\hline $\mathrm{H}$ & 0.26215 & 0.36027 & 0.38672 \\
\hline $\mathrm{H}$ & 1.21724 & 0.305 & 0.36827 \\
\hline $\mathrm{H}$ & 1.21886 & 0.27508 & 0.37068 \\
\hline $\mathrm{H}$ & 0.27133 & 0.2776 & 0.36488 \\
\hline $\mathrm{H}$ & 0.26985 & 0.30746 & 0.36208 \\
\hline $\mathrm{H}$ & 0.61423 & 0.38664 & 0.73114 \\
\hline $\mathrm{H}$ & 0.58745 & 0.42619 & 0.61374 \\
\hline $\mathrm{H}$ & 0.61559 & 0.42387 & 0.71385 \\
\hline $\mathrm{H}$ & 0.59848 & 0.43767 & 0.74553 \\
\hline $\mathrm{H}$ & 0.55833 & 0.40564 & 0.72664 \\
\hline $\mathrm{H}$ & 0.57536 & 0.39214 & 0.69518 \\
\hline $\mathrm{H}$ & 0.54426 & 0.42594 & 0.74376 \\
\hline $\mathrm{H}$ & 0.51901 & 0.43434 & 0.76517 \\
\hline $\mathrm{H}$ & 0.50459 & 0.44773 & 0.7997 \\
\hline $\mathrm{H}$ & 0.55086 & 0.46274 & 0.83132 \\
\hline $\mathrm{H}$ & 0.56523 & 0.44896 & 0.79723 \\
\hline $\mathrm{H}$ & 0.49803 & 0.47385 & 0.81649 \\
\hline $\mathrm{H}$ & 0.48388 & 0.4874 & 0.85115 \\
\hline $\mathrm{H}$ & 0.52108 & 0.47084 & 0.90251 \\
\hline $\mathrm{H}$ & 0.53529 & 0.45716 & 0.868 \\
\hline $\mathrm{H}$ & 1.11205 & -0.11309 & 0.21827 \\
\hline $\mathrm{H}$ & 1.08394 & -0.07982 & 1.09705 \\
\hline $\mathrm{H}$ & 1.11401 & -0.07725 & 0.19723 \\
\hline $\mathrm{H}$ & 1.09747 & -0.06184 & 0.22785 \\
\hline $\mathrm{H}$ & 1.05647 & -0.09363 & 0.21268 \\
\hline $\mathrm{H}$ & 1.07291 & -0.10871 & 0.18221 \\
\hline $\mathrm{H}$ & 1.04303 & -0.07217 & 0.22735 \\
\hline $\mathrm{H}$ & 1.01813 & -0.06076 & 0.24617 \\
\hline $\mathrm{H}$ & 1.00418 & -0.04437 & 0.27807 \\
\hline $\mathrm{H}$ & 1.05083 & -0.03176 & 0.31077 \\
\hline $\mathrm{H}$ & 1.06472 & -0.04832 & 0.27919 \\
\hline $\mathrm{H}$ & 1.00044 & -0.01628 & 0.29021 \\
\hline $\mathrm{H}$ & 0.98662 & 0.0004 & 0.32182 \\
\hline $\mathrm{H}$ & 1.0194 & -0.01786 & 0.37866 \\
\hline
\end{tabular}




\begin{tabular}{|c|c|c|c|}
\hline $\mathrm{H}$ & 1.03333 & -0.03461 & 0.3471 \\
\hline $\mathrm{H}$ & 1.19579 & 0.46648 & 0.38134 \\
\hline $\mathrm{H}$ & 0.30566 & 0.46878 & 0.3826 \\
\hline $\mathrm{H}$ & 0.3142 & 0.4944 & 0.34602 \\
\hline $\mathrm{H}$ & 0.34421 & 0.49639 & 0.34875 \\
\hline $\mathrm{H}$ & 0.34013 & -0.48898 & 0.42629 \\
\hline $\mathrm{H}$ & 0.31029 & -0.49126 & 0.42363 \\
\hline $\mathrm{H}$ & 0.36417 & -0.47651 & 0.41497 \\
\hline $\mathrm{H}$ & 0.39078 & -0.46367 & 0.42499 \\
\hline $\mathrm{H}$ & 0.42078 & -0.46414 & 0.42562 \\
\hline $\mathrm{H}$ & 0.42026 & 0.49238 & 0.37956 \\
\hline $\mathrm{H}$ & 0.39046 & 0.49275 & 0.37941 \\
\hline $\mathrm{H}$ & 0.4442 & -0.46023 & 0.39827 \\
\hline $\mathrm{H}$ & 0.47417 & -0.46133 & 0.39709 \\
\hline $\mathrm{H}$ & 0.47252 & 0.48633 & 0.40605 \\
\hline $\mathrm{H}$ & 0.44264 & 0.48729 & 0.40764 \\
\hline $\mathrm{H}$ & 0.63694 & -0.36397 & 0.23028 \\
\hline $\mathrm{H}$ & 0.67152 & -0.33602 & 1.10993 \\
\hline $\mathrm{H}$ & 0.67502 & -0.36613 & 0.20828 \\
\hline $\mathrm{H}$ & 0.69003 & -0.34964 & 0.23943 \\
\hline $\mathrm{H}$ & 0.65614 & -0.30986 & 0.22767 \\
\hline $\mathrm{H}$ & 0.64146 & -0.32626 & 0.19665 \\
\hline $\mathrm{H}$ & 0.6771 & -0.29577 & 0.24269 \\
\hline $\mathrm{H}$ & 0.68698 & -0.27078 & 0.26289 \\
\hline $\mathrm{H}$ & 0.70287 & -0.25652 & 0.29497 \\
\hline $\mathrm{H}$ & 0.71936 & -0.30298 & 0.32416 \\
\hline $\mathrm{H}$ & 0.70315 & -0.3172 & 0.29242 \\
\hline $\mathrm{H}$ & 0.73044 & -0.25061 & 0.30638 \\
\hline $\mathrm{H}$ & 0.74633 & -0.2361 & 0.33821 \\
\hline $\mathrm{H}$ & 0.73271 & -0.27222 & 0.39332 \\
\hline $\mathrm{H}$ & 0.71673 & -0.28682 & 0.36159 \\
\hline $\mathrm{H}$ & 1.13486 & 0.1356 & 0.71972 \\
\hline $\mathrm{H}$ & 1.17097 & 0.16707 & 0.60248 \\
\hline $\mathrm{H}$ & 1.17177 & 0.1342 & 0.70001 \\
\hline $\mathrm{H}$ & 1.18705 & 0.15014 & 0.73154 \\
\hline $\mathrm{H}$ & 1.15493 & 0.19122 & 0.71865 \\
\hline $\mathrm{H}$ & 1.14003 & 0.1754 & 0.6872 \\
\hline $\mathrm{H}$ & 1.17697 & 0.20445 & 0.73174 \\
\hline $\mathrm{H}$ & 1.1889 & 0.22935 & 0.74907 \\
\hline $\mathrm{H}$ & 1.20597 & 0.24371 & 0.77955 \\
\hline $\mathrm{H}$ & 1.21848 & 0.19758 & 0.81411 \\
\hline $\mathrm{H}$ & 1.20115 & 0.18326 & 0.78387 \\
\hline
\end{tabular}




\begin{tabular}{|r|r|r|r|}
\hline $\mathrm{H}$ & 1.23429 & 0.24733 & 0.78997 \\
\hline $\mathrm{H}$ & 1.25137 & 0.26208 & 0.82002 \\
\hline $\mathrm{H}$ & 1.234 & 0.23092 & 0.87962 \\
\hline $\mathrm{H}$ & 1.21687 & 0.21605 & 0.84961 \\
\hline $\mathrm{H}$ & 0.71786 & 0.05511 & 0.87973 \\
\hline $\mathrm{H}$ & 0.71744 & -0.0549 & 0.87983 \\
\hline $\mathrm{H}$ & 0.7436 & -0.06285 & 0.84141 \\
\hline $\mathrm{H}$ & 0.74335 & -0.09299 & 0.84242 \\
\hline $\mathrm{H}$ & 0.75567 & -0.09179 & 0.92116 \\
\hline $\mathrm{H}$ & 0.75572 & -0.06183 & 0.9201 \\
\hline $\mathrm{H}$ & 0.76584 & -0.11654 & 0.91036 \\
\hline $\mathrm{H}$ & 0.77594 & -0.14407 & 0.921 \\
\hline $\mathrm{H}$ & 0.77406 & -0.17391 & 0.91871 \\
\hline $\mathrm{H}$ & 0.73422 & -0.16964 & 0.86594 \\
\hline $\mathrm{H}$ & 0.73603 & -0.13984 & 0.86838 \\
\hline $\mathrm{H}$ & 0.77924 & -0.19597 & 0.88872 \\
\hline $\mathrm{H}$ & 0.7775 & -0.22588 & 0.88662 \\
\hline $\mathrm{H}$ & 0.72495 & -0.22303 & 0.8895 \\
\hline $\mathrm{H}$ & 0.72654 & -0.19311 & 0.89177 \\
\hline
\end{tabular}

Table S8. Fractional atomic coordinates for the unit cell of COF-1 with the interpenetrated tbo topology.

\begin{tabular}{|c|c|c|c|}
\hline \multicolumn{4}{|c|}{ Space group: $F \mathrm{~d}-3 \mathrm{~m}$} \\
\hline \multicolumn{4}{|c|}{$\mathrm{a}=\mathrm{b}=\mathrm{c}=96.1389 \AA, \alpha=\beta=\gamma=90^{\circ}$} \\
\hline $\mathrm{C}$ & 0.29302 & 1.02022 & 0.53805 \\
\hline $\mathrm{C}$ & 0.30344 & 1.02739 & 0.54521 \\
\hline $\mathrm{C}$ & 0.33405 & 1.07565 & 0.55783 \\
\hline $\mathrm{C}$ & 0.34526 & 1.0822 & 0.56437 \\
\hline $\mathrm{C}$ & 0.21029 & 0.97981 & 0.46198 \\
\hline $\mathrm{C}$ & 0.19973 & 0.97274 & 0.45492 \\
\hline $\mathrm{C}$ & 0.1539 & 0.93631 & 0.45413 \\
\hline $\mathrm{C}$ & 0.14295 & 0.92955 & 0.44738 \\
\hline $\mathrm{H}$ & 0.28907 & 1.01054 & 0.54222 \\
\hline $\mathrm{H}$ & 0.30749 & 1.02321 & 0.55484 \\
\hline $\mathrm{H}$ & 0.32981 & 1.08005 & 0.54837 \\
\hline $\mathrm{H}$ & 0.34958 & 1.09159 & 0.55991 \\
\hline $\mathrm{H}$ & 0.21428 & 0.98946 & 0.45778 \\
\hline $\mathrm{H}$ & 0.19564 & 0.97696 & 0.44533 \\
\hline $\mathrm{H}$ & 0.15802 & 0.93201 & 0.46369 \\
\hline
\end{tabular}




\begin{tabular}{|c|c|c|c|}
\hline $\mathrm{H}$ & 0.1387 & 0.9201 & 0.45178 \\
\hline $\mathrm{N}$ & 0.25 & 1.02261 & 0.25 \\
\hline $\mathrm{C}$ & 0.24048 & 1.02086 & 0.52086 \\
\hline $\mathrm{C}$ & 0.25877 & 1.03062 & 0.53062 \\
\hline $\mathrm{C}$ & 0.28779 & 1.02552 & 0.52552 \\
\hline $\mathrm{C}$ & 0.30864 & 1.03991 & 0.53991 \\
\hline $\mathrm{N}$ & 0.31951 & 1.04706 & 0.54706 \\
\hline $\mathrm{C}$ & 0.31693 & 1.05641 & 0.55641 \\
\hline $\mathrm{C}$ & 0.32846 & 1.06342 & 0.56342 \\
\hline $\mathrm{C}$ & 0.35092 & 1.07655 & 0.57655 \\
\hline $\mathrm{C}$ & 0.36294 & 1.08321 & 0.58321 \\
\hline $\mathrm{C}$ & 0.38595 & 1.09586 & 0.59586 \\
\hline $\mathrm{C}$ & 0.27687 & 1.01782 & 0.51782 \\
\hline $\mathrm{C}$ & 0.26288 & 1.02086 & 0.52086 \\
\hline $\mathrm{C}$ & 0.24459 & 1.03062 & 0.53062 \\
\hline $\mathrm{N}$ & 0.25168 & 1.01482 & 0.51482 \\
\hline $\mathrm{C}$ & 0.21556 & 0.97448 & 0.47448 \\
\hline $\mathrm{C}$ & 0.19437 & 0.96033 & 0.46033 \\
\hline $\mathrm{N}$ & 0.18336 & 0.95329 & 0.45329 \\
\hline $\mathrm{C}$ & 0.17045 & 0.956 & 0.456 \\
\hline $\mathrm{C}$ & 0.15938 & 0.94863 & 0.44863 \\
\hline $\mathrm{C}$ & 0.1374 & 0.93509 & 0.43509 \\
\hline $\mathrm{C}$ & 0.12551 & 0.92831 & 0.42831 \\
\hline $\mathrm{C}$ & 0.10272 & 0.91546 & 0.41546 \\
\hline $\mathrm{C}$ & 0.22649 & 0.98218 & 0.48218 \\
\hline $\mathrm{H}$ & 0.26531 & 1.0371 & 0.5371 \\
\hline $\mathrm{H}$ & 0.30633 & 1.05912 & 0.55912 \\
\hline $\mathrm{H}$ & 0.23805 & 1.0371 & 0.5371 \\
\hline $\mathrm{H}$ & 0.16768 & 0.96373 & 0.46373 \\
\hline $\mathrm{N}$ & 0.39767 & 1.10233 & 0.60233 \\
\hline $\mathrm{N}$ & 0.09111 & 0.90889 & 0.40889 \\
\hline $\mathrm{C}$ & 0.63903 & 1.09342 & 0.40658 \\
\hline $\mathrm{C}$ & 0.62755 & 1.09973 & 0.40027 \\
\hline $\mathrm{C}$ & 0.62355 & 1.07935 & 0.42065 \\
\hline $\mathrm{C}$ & 0.61209 & 1.08566 & 0.41434 \\
\hline $\mathrm{C}$ & 0.04496 & 1.24499 & 0.25501 \\
\hline $\mathrm{H}$ & 0.64942 & 1.09648 & 0.40352 \\
\hline $\mathrm{H}$ & 0.62913 & 1.10761 & 0.39239 \\
\hline $\mathrm{H}$ & 0.62191 & 1.07147 & 0.42853 \\
\hline $\mathrm{H}$ & 0.6017 & 1.08261 & 0.41739 \\
\hline $\mathrm{H}$ & 0.05412 & 1.24037 & 0.25963 \\
\hline $\mathrm{C}$ & 0.66172 & 0.66172 & 0.63371 \\
\hline
\end{tabular}




\begin{tabular}{|l|r|r|r|}
\hline C & 0.66812 & 0.66812 & 0.62234 \\
\hline C & 0.68205 & 0.68205 & 0.63806 \\
\hline C & 0.67565 & 0.67565 & 0.64941 \\
\hline H & 0.65385 & 0.65385 & 0.632 \\
\hline H & 0.66515 & 0.66515 & 0.6119 \\
\hline H & 0.68992 & 0.68992 & 0.63984 \\
\hline H & 0.67862 & 0.67862 & 0.65985 \\
\hline C & 0.24208 & 1.03116 & 0.25792 \\
\hline C & 0.49208 & 1.00792 & 0.7778 \\
\hline C & 0.49499 & 1.00501 & 0.7916 \\
\hline H & 0.49037 & 1.00963 & 0.80076 \\
\hline N & 0.5 & 1 & 0.76925 \\
\hline
\end{tabular}

Table S9. Fractional atomic coordinates for the unit cell of COF-2 with the noninterpenetrated pto topology.

\begin{tabular}{|c|c|c|c|}
\hline \multicolumn{4}{|c|}{ Space group: $P-43 \mathrm{n}$} \\
\hline \multicolumn{4}{|c|}{$\mathrm{a}=\mathrm{b}=\mathrm{c}=55.7606 \AA$} \\
\hline Atom & $\mathrm{x}$ & $\mathrm{y}$ & $\mathrm{z}$ \\
\hline $\mathrm{C}$ & -0.01442 & 0.54794 & 0.23769 \\
\hline $\mathrm{N}$ & 0.00335 & 0.53149 & 0.23777 \\
\hline $\mathrm{C}$ & 0.02393 & 0.54423 & 0.23861 \\
\hline $\mathrm{C}$ & 0.01935 & 0.56782 & 0.23221 \\
\hline $\mathrm{C}$ & -0.00512 & 0.57018 & 0.23157 \\
\hline $\mathrm{C}$ & 0.04504 & 0.53696 & 0.25087 \\
\hline $\mathrm{C}$ & 0.06542 & 0.55405 & 0.25182 \\
\hline $\mathrm{C}$ & 0.07189 & 0.56478 & 0.27359 \\
\hline $\mathrm{C}$ & 0.09093 & 0.58101 & 0.27449 \\
\hline $\mathrm{C}$ & 0.10397 & 0.58657 & 0.25377 \\
\hline $\mathrm{C}$ & 0.09735 & 0.5759 & 0.23184 \\
\hline $\mathrm{C}$ & 0.07824 & 0.55971 & 0.23095 \\
\hline $\mathrm{N}$ & 0.1231 & 0.60373 & 0.25565 \\
\hline $\mathrm{C}$ & 0.14024 & 0.60698 & 0.24022 \\
\hline $\mathrm{C}$ & 0.15853 & 0.62573 & 0.24408 \\
\hline $\mathrm{C}$ & 0.17731 & 0.62829 & 0.22756 \\
\hline $\mathrm{C}$ & 0.19462 & 0.64613 & 0.23065 \\
\hline $\mathrm{C}$ & 0.19348 & 0.66182 & 0.25034 \\
\hline $\mathrm{C}$ & 0.17471 & 0.65915 & 0.26695 \\
\hline $\mathrm{C}$ & 0.15742 & 0.64129 & 0.26387 \\
\hline $\mathrm{C}$ & 0.21159 & 0.6811 & 0.2534 \\
\hline
\end{tabular}




\begin{tabular}{|c|c|c|c|}
\hline $\mathrm{C}$ & 0.21988 & 0.69414 & 0.23349 \\
\hline $\mathrm{C}$ & 0.23659 & 0.71256 & 0.23639 \\
\hline $\mathrm{C}$ & 0.24537 & 0.71835 & 0.25925 \\
\hline $\mathrm{C}$ & 0.23723 & 0.70527 & 0.27915 \\
\hline $\mathrm{C}$ & 0.22052 & 0.68683 & 0.27626 \\
\hline $\mathrm{H}$ & 0.03185 & 0.58266 & 0.23217 \\
\hline $\mathrm{H}$ & -0.01454 & 0.58713 & 0.23101 \\
\hline $\mathrm{H}$ & 0.06207 & 0.56067 & 0.28983 \\
\hline $\mathrm{H}$ & 0.09564 & 0.58926 & 0.29141 \\
\hline $\mathrm{H}$ & 0.10645 & 0.58026 & 0.21532 \\
\hline $\mathrm{H}$ & 0.07332 & 0.55166 & 0.21398 \\
\hline $\mathrm{H}$ & 0.14171 & 0.59576 & 0.22448 \\
\hline $\mathrm{H}$ & 0.17858 & 0.61647 & 0.21221 \\
\hline $\mathrm{H}$ & 0.20902 & 0.64752 & 0.21773 \\
\hline $\mathrm{H}$ & 0.17313 & 0.67115 & 0.28211 \\
\hline $\mathrm{H}$ & 0.14309 & 0.63982 & 0.27687 \\
\hline $\mathrm{H}$ & 0.21308 & 0.69042 & 0.21569 \\
\hline $\mathrm{H}$ & 0.24241 & 0.72258 & 0.22083 \\
\hline $\mathrm{H}$ & 0.24393 & 0.7093 & 0.29691 \\
\hline $\mathrm{H}$ & 0.21477 & 0.6769 & 0.29191 \\
\hline $\mathrm{N}$ & 0.2623 & 0.2623 & 0.7377 \\
\hline $\mathrm{Cu}$ & 0.5 & 1.25 & 1 \\
\hline
\end{tabular}

Table S10. Fractional atomic coordinates for the unit cell of COF-2 with the noninterpenetrated fjh topology.

\begin{tabular}{|c|c|c|c|}
\hline \multicolumn{4}{|c|}{ Space group: $P 42 / \mathrm{ncm}$} \\
\hline \multicolumn{4}{|c|}{$\mathrm{a}=\mathrm{b}=81.6412 \AA, \mathrm{c}=52.6399 \AA, \alpha=\beta=\gamma=90^{\circ}$} \\
\hline Atom & $\mathrm{x}$ & $\mathrm{y}$ & $\mathrm{z}$ \\
\hline $\mathrm{C}$ & 0.12081 & 0.36056 & 0.44638 \\
\hline $\mathrm{C}$ & 0.13917 & 0.37271 & 0.42201 \\
\hline $\mathrm{C}$ & 0.141 & 0.40386 & 0.51575 \\
\hline $\mathrm{C}$ & 0.14919 & 0.41796 & 0.50833 \\
\hline $\mathrm{C}$ & 0.14445 & 0.39547 & 0.45177 \\
\hline $\mathrm{C}$ & 0.15415 & 0.40425 & 0.43215 \\
\hline $\mathrm{C}$ & 0.1476 & 0.41794 & 0.41968 \\
\hline $\mathrm{C}$ & 0.15691 & 0.42636 & 0.40162 \\
\hline $\mathrm{C}$ & 0.17291 & 0.42136 & 0.39593 \\
\hline $\mathrm{C}$ & 0.17933 & 0.40739 & 0.40811 \\
\hline $\mathrm{C}$ & 0.17002 & 0.39897 & 0.42613 \\
\hline
\end{tabular}




\begin{tabular}{|c|c|c|c|}
\hline $\mathrm{N}$ & 0.18203 & 0.43058 & 0.37745 \\
\hline $\mathrm{C}$ & 0.19783 & 0.43026 & 0.37445 \\
\hline $\mathrm{C}$ & 0.20587 & 0.44029 & 0.35473 \\
\hline $\mathrm{C}$ & 0.223 & 0.4401 & 0.3528 \\
\hline $\mathrm{C}$ & 0.23096 & 0.44957 & 0.33446 \\
\hline $\mathrm{C}$ & 0.22193 & 0.45941 & 0.31761 \\
\hline $\mathrm{C}$ & 0.20475 & 0.45952 & 0.31946 \\
\hline $\mathrm{C}$ & 0.19679 & 0.45007 & 0.33786 \\
\hline $\mathrm{C}$ & 0.23045 & 0.46952 & 0.29825 \\
\hline $\mathrm{C}$ & 0.24493 & 0.47793 & 0.30446 \\
\hline $\mathrm{C}$ & 0.25314 & 0.48721 & 0.28617 \\
\hline $\mathrm{C}$ & 0.24706 & 0.48829 & 0.26127 \\
\hline $\mathrm{C}$ & 0.23254 & 0.47999 & 0.25506 \\
\hline $\mathrm{C}$ & 0.22429 & 0.47072 & 0.27337 \\
\hline $\mathrm{C}$ & 0.62065 & 0.86072 & -0.04445 \\
\hline $\mathrm{C}$ & 0.63894 & 0.87295 & -0.06886 \\
\hline $\mathrm{C}$ & 0.64108 & 0.90379 & 0.0251 \\
\hline $\mathrm{C}$ & 0.64924 & 0.91792 & 0.01772 \\
\hline $\mathrm{C}$ & 0.64429 & 0.89562 & -0.03897 \\
\hline $\mathrm{C}$ & 0.65391 & 0.90448 & -0.05859 \\
\hline $\mathrm{C}$ & 0.64723 & 0.9181 & -0.07106 \\
\hline $\mathrm{C}$ & 0.65651 & 0.92669 & -0.08896 \\
\hline $\mathrm{C}$ & 0.67262 & 0.92194 & -0.09445 \\
\hline $\mathrm{C}$ & 0.67916 & 0.908 & -0.08234 \\
\hline $\mathrm{C}$ & 0.66986 & 0.8994 & -0.06449 \\
\hline $\mathrm{N}$ & 0.68176 & 0.9314 & -0.11259 \\
\hline $\mathrm{C}$ & 0.69761 & 0.93153 & -0.1149 \\
\hline $\mathrm{C}$ & 0.70572 & 0.94188 & -0.13416 \\
\hline $\mathrm{C}$ & 0.72288 & 0.9426 & -0.13479 \\
\hline $\mathrm{C}$ & 0.73089 & 0.95248 & -0.15253 \\
\hline $\mathrm{C}$ & 0.72189 & 0.96182 & -0.17009 \\
\hline $\mathrm{C}$ & 0.70469 & 0.96098 & -0.16961 \\
\hline $\mathrm{C}$ & 0.69668 & 0.95112 & -0.15178 \\
\hline $\mathrm{C}$ & 0.73045 & 0.97246 & -0.18869 \\
\hline $\mathrm{C}$ & 0.74406 & 0.98182 & -0.18119 \\
\hline $\mathrm{C}$ & 0.75227 & 0.9917 & -0.19869 \\
\hline $\mathrm{C}$ & 0.74709 & 0.99242 & -0.22411 \\
\hline $\mathrm{C}$ & 0.73343 & 0.98314 & -0.23162 \\
\hline $\mathrm{C}$ & 0.72517 & 0.97329 & -0.21408 \\
\hline $\mathrm{N}$ & 0.13369 & 0.39699 & 0.49532 \\
\hline $\mathrm{C}$ & 0.54176 & 0.47001 & 0.74983 \\
\hline $\mathrm{C}$ & 0.53369 & 0.48494 & 0.74964 \\
\hline
\end{tabular}




\begin{tabular}{|c|c|c|c|}
\hline $\mathrm{C}$ & 0.5 & 0.45814 & 0.75061 \\
\hline $\mathrm{C}$ & 0.48494 & 0.4663 & 0.75027 \\
\hline $\mathrm{C}$ & 0.45824 & 0.47001 & 0.74974 \\
\hline $\mathrm{C}$ & 0.43993 & 0.50003 & 0.74882 \\
\hline $\mathrm{C}$ & 0.43098 & 0.49633 & 0.77081 \\
\hline $\mathrm{C}$ & 0.41386 & 0.49643 & 0.77026 \\
\hline $\mathrm{C}$ & 0.40547 & 0.50053 & 0.74794 \\
\hline $\mathrm{C}$ & 0.41449 & 0.50405 & 0.72579 \\
\hline $\mathrm{C}$ & 0.4316 & 0.50379 & 0.72628 \\
\hline $\mathrm{N}$ & 0.38788 & 0.50048 & 0.74829 \\
\hline $\mathrm{C}$ & 0.37851 & 0.50866 & 0.73288 \\
\hline $\mathrm{C}$ & 0.3605 & 0.50756 & 0.73475 \\
\hline $\mathrm{C}$ & 0.35076 & 0.51819 & 0.72028 \\
\hline $\mathrm{C}$ & 0.33369 & 0.51741 & 0.72174 \\
\hline $\mathrm{C}$ & 0.32603 & 0.50586 & 0.73755 \\
\hline $\mathrm{C}$ & 0.33579 & 0.49514 & 0.75195 \\
\hline $\mathrm{C}$ & 0.35287 & 0.49602 & 0.75065 \\
\hline $\mathrm{C}$ & 0.30792 & 0.50496 & 0.73896 \\
\hline $\mathrm{C}$ & 0.29852 & 0.51933 & 0.74082 \\
\hline $\mathrm{C}$ & 0.28145 & 0.51848 & 0.74207 \\
\hline $\mathrm{C}$ & 0.27345 & 0.50327 & 0.74127 \\
\hline $\mathrm{C}$ & 0.28282 & 0.48891 & 0.73938 \\
\hline $\mathrm{C}$ & 0.2999 & 0.48973 & 0.73834 \\
\hline $\mathrm{N}$ & 0.25582 & 0.49758 & 0.24227 \\
\hline $\mathrm{H}$ & 0.14472 & 0.37781 & 0.4051 \\
\hline $\mathrm{H}$ & 0.15681 & 0.4258 & 0.51996 \\
\hline $\mathrm{H}$ & 0.13532 & 0.42211 & 0.42402 \\
\hline $\mathrm{H}$ & 0.15172 & 0.43695 & 0.39222 \\
\hline $\mathrm{H}$ & 0.1914 & 0.40277 & 0.40365 \\
\hline $\mathrm{H}$ & 0.17517 & 0.38833 & 0.43547 \\
\hline $\mathrm{H}$ & 0.2055 & 0.423 & 0.38688 \\
\hline $\mathrm{H}$ & 0.23026 & 0.43262 & 0.36553 \\
\hline $\mathrm{H}$ & 0.24419 & 0.44903 & 0.33328 \\
\hline $\mathrm{H}$ & 0.19748 & 0.46711 & 0.30692 \\
\hline $\mathrm{H}$ & 0.18355 & 0.45047 & 0.33891 \\
\hline $\mathrm{H}$ & 0.24984 & 0.47746 & 0.32355 \\
\hline $\mathrm{H}$ & 0.26422 & 0.49363 & 0.29147 \\
\hline $\mathrm{H}$ & 0.22772 & 0.48048 & 0.23592 \\
\hline $\mathrm{H}$ & 0.21328 & 0.46421 & 0.26794 \\
\hline $\mathrm{H}$ & 0.64444 & 0.87811 & -0.08577 \\
\hline $\mathrm{H}$ & 0.65691 & 0.92571 & 0.02934 \\
\hline $\mathrm{H}$ & 0.63489 & 0.92211 & -0.0668 \\
\hline
\end{tabular}




\begin{tabular}{|c|c|c|c|}
\hline $\mathrm{H}$ & 0.65124 & 0.93725 & -0.09833 \\
\hline $\mathrm{H}$ & 0.69131 & 0.90358 & -0.08669 \\
\hline $\mathrm{H}$ & 0.67511 & 0.88881 & -0.05515 \\
\hline $\mathrm{H}$ & 0.70524 & 0.92453 & -0.10208 \\
\hline $\mathrm{H}$ & 0.73012 & 0.93554 & -0.12147 \\
\hline $\mathrm{H}$ & 0.74415 & 0.9527 & -0.15267 \\
\hline $\mathrm{H}$ & 0.69743 & 0.96815 & -0.18274 \\
\hline $\mathrm{H}$ & 0.68342 & 0.95081 & -0.15174 \\
\hline $\mathrm{H}$ & 0.74825 & 0.98164 & -0.16168 \\
\hline $\mathrm{H}$ & 0.76264 & 0.99888 & -0.19237 \\
\hline $\mathrm{H}$ & 0.72932 & 0.98335 & -0.25116 \\
\hline $\mathrm{H}$ & 0.71484 & 0.96606 & -0.22048 \\
\hline $\mathrm{H}$ & 0.55482 & 0.46778 & 0.74979 \\
\hline $\mathrm{H}$ & 0.44518 & 0.46777 & 0.74942 \\
\hline $\mathrm{H}$ & 0.43726 & 0.49333 & 0.78832 \\
\hline $\mathrm{H}$ & 0.40709 & 0.4935 & 0.78735 \\
\hline $\mathrm{H}$ & 0.40842 & 0.50672 & 0.70801 \\
\hline $\mathrm{H}$ & 0.43838 & 0.50653 & 0.70912 \\
\hline $\mathrm{H}$ & 0.38376 & 0.51688 & 0.71896 \\
\hline $\mathrm{H}$ & 0.35637 & 0.52715 & 0.70784 \\
\hline $\mathrm{H}$ & 0.32646 & 0.52569 & 0.71024 \\
\hline $\mathrm{H}$ & 0.3302 & 0.48622 & 0.76446 \\
\hline $\mathrm{H}$ & 0.36008 & 0.48764 & 0.76202 \\
\hline $\mathrm{H}$ & 0.30439 & 0.53121 & 0.74157 \\
\hline $\mathrm{H}$ & 0.27442 & 0.52969 & 0.74344 \\
\hline $\mathrm{H}$ & 0.27686 & 0.47707 & 0.73882 \\
\hline $\mathrm{H}$ & 0.30684 & 0.47848 & 0.73668 \\
\hline $\mathrm{N}$ & 0.13021 & 0.36979 & 0.4619 \\
\hline $\mathrm{N}$ & 0.3699 & 0.1301 & -0.02894 \\
\hline $\mathrm{N}$ & 0.51722 & 0.48278 & 0.75 \\
\hline $\mathrm{N}$ & 0.48277 & 0.48277 & 0.75 \\
\hline $\mathrm{Cu}$ & 0.87579 & 0.62421 & 0.49537 \\
\hline $\mathrm{Cu}$ & 1 & 1 & 0.75 \\
\hline
\end{tabular}

Table S11. Fractional atomic coordinates for the unit cell of COF-2 with the noninterpenetrated ffc topology.

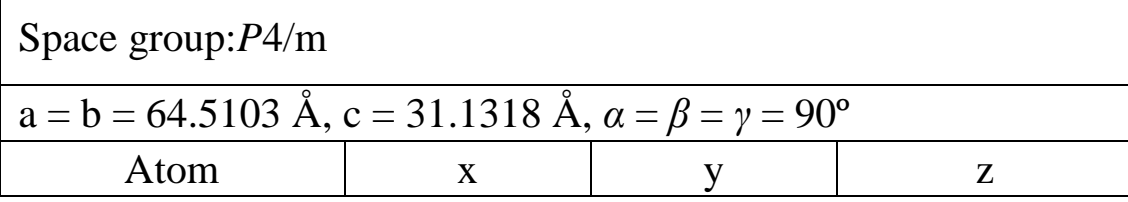




\begin{tabular}{|c|c|c|c|}
\hline $\mathrm{C}$ & 0.41331 & 0.49511 & 0.53895 \\
\hline $\mathrm{C}$ & 0.39168 & 0.49392 & 0.53892 \\
\hline $\mathrm{C}$ & 0.31373 & 0.50535 & 0.46133 \\
\hline $\mathrm{C}$ & 0.05345 & 0.49556 & 0.11136 \\
\hline $\mathrm{C}$ & 0.06356 & 0.51349 & 0.12564 \\
\hline $\mathrm{C}$ & 0.07803 & 0.51232 & 0.15893 \\
\hline $\mathrm{C}$ & 0.08248 & 0.49332 & 0.17866 \\
\hline $\mathrm{C}$ & 0.07268 & 0.47523 & 0.16342 \\
\hline $\mathrm{C}$ & 0.05823 & 0.47641 & 0.13009 \\
\hline $\mathrm{N}$ & 0.09689 & 0.49321 & 0.21388 \\
\hline $\mathrm{C}$ & 0.09961 & 0.47827 & 0.24132 \\
\hline $\mathrm{C}$ & 0.11448 & 0.48046 & 0.27708 \\
\hline $\mathrm{C}$ & 0.11584 & 0.46475 & 0.30804 \\
\hline $\mathrm{C}$ & 0.12918 & 0.46679 & 0.34312 \\
\hline $\mathrm{C}$ & 0.1415 & 0.48461 & 0.34792 \\
\hline $\mathrm{C}$ & 0.14037 & 0.50021 & 0.31647 \\
\hline $\mathrm{C}$ & 0.12699 & 0.49817 & 0.28141 \\
\hline $\mathrm{C}$ & 0.15501 & 0.48714 & 0.38619 \\
\hline $\mathrm{C}$ & 0.16602 & 0.47016 & 0.40304 \\
\hline $\mathrm{C}$ & 0.17801 & 0.4724 & 0.44012 \\
\hline $\mathrm{C}$ & 0.17923 & 0.49161 & 0.46082 \\
\hline $\mathrm{C}$ & 0.16858 & 0.50865 & 0.44394 \\
\hline $\mathrm{C}$ & 0.15655 & 0.50646 & 0.40691 \\
\hline $\mathrm{C}$ & 0.29217 & 0.50336 & 0.53881 \\
\hline $\mathrm{C}$ & 0.24583 & 0.49902 & 0.5387 \\
\hline $\mathrm{C}$ & 0.22428 & 0.49693 & 0.53864 \\
\hline $\mathrm{C}$ & 0.50138 & 0.01661 & 0.90969 \\
\hline $\mathrm{C}$ & 0.50353 & 0.04349 & 0.03453 \\
\hline $\mathrm{C}$ & 0.50528 & 0.06399 & 0.02184 \\
\hline $\mathrm{C}$ & 0.0105 & 0.49915 & 0.86707 \\
\hline $\mathrm{C}$ & 0.96269 & 0.50307 & 0.07752 \\
\hline $\mathrm{H}$ & 0.42157 & 0.49555 & 0.56919 \\
\hline $\mathrm{H}$ & 0.38337 & 0.49342 & 0.5691 \\
\hline $\mathrm{H}$ & 0.32199 & 0.50613 & 0.43108 \\
\hline $\mathrm{H}$ & 0.06003 & 0.52838 & 0.11134 \\
\hline $\mathrm{H}$ & 0.08555 & 0.52634 & 0.16998 \\
\hline $\mathrm{H}$ & 0.07609 & 0.46016 & 0.17676 \\
\hline $\mathrm{H}$ & 0.0506 & 0.46243 & 0.11907 \\
\hline $\mathrm{H}$ & 0.09035 & 0.46434 & 0.23981 \\
\hline $\mathrm{H}$ & 0.10632 & 0.45098 & 0.30545 \\
\hline $\mathrm{H}$ & 0.12947 & 0.45463 & 0.36705 \\
\hline $\mathrm{H}$ & 0.14987 & 0.51398 & 0.31892 \\
\hline
\end{tabular}




\begin{tabular}{|c|c|c|c|}
\hline $\mathrm{H}$ & 0.12636 & 0.51051 & 0.2579 \\
\hline $\mathrm{H}$ & 0.16538 & 0.45516 & 0.38753 \\
\hline $\mathrm{H}$ & 0.18623 & 0.45916 & 0.45301 \\
\hline $\mathrm{H}$ & 0.16943 & 0.52356 & 0.45973 \\
\hline $\mathrm{H}$ & 0.14806 & 0.51976 & 0.39512 \\
\hline $\mathrm{H}$ & 0.28499 & 0.5027 & 0.56994 \\
\hline $\mathrm{H}$ & 0.25301 & 0.49977 & 0.56982 \\
\hline $\mathrm{H}$ & 0.2164 & 0.4963 & 0.56927 \\
\hline $\mathrm{H}$ & 0.5065 & 0.07759 & 0.04201 \\
\hline $\mathrm{H}$ & 0.02018 & 0.49838 & 0.83873 \\
\hline $\mathrm{C}$ & 0.49702 & 0.55284 & 0.5 \\
\hline $\mathrm{C}$ & 0.47858 & 0.54148 & 0.5 \\
\hline $\mathrm{C}$ & 0.45916 & 0.55062 & 0.5 \\
\hline $\mathrm{C}$ & 0.4242 & 0.49573 & 0.5 \\
\hline $\mathrm{C}$ & 0.38082 & 0.49341 & 0.5 \\
\hline $\mathrm{N}$ & 0.35873 & 0.49204 & 0.5 \\
\hline $\mathrm{C}$ & 0.2807 & 0.50229 & 0.5 \\
\hline $\mathrm{C}$ & 0.25728 & 0.50008 & 0.5 \\
\hline $\mathrm{C}$ & 0.21314 & 0.49573 & 0.5 \\
\hline $\mathrm{C}$ & 0.45637 & 0.51662 & 0.5 \\
\hline $\mathrm{C}$ & 0.44512 & 0.5349 & 0.5 \\
\hline $\mathrm{N}$ & 0.47702 & 0.52053 & 0.5 \\
\hline $\mathrm{N}$ & 0.19076 & 0.49363 & 0.5 \\
\hline $\mathrm{C}$ & 0.34715 & 0.50843 & 0.5 \\
\hline $\mathrm{C}$ & 0.32456 & 0.50631 & 0.5 \\
\hline $\mathrm{H}$ & 0.45545 & 0.56698 & 0.5 \\
\hline $\mathrm{H}$ & 0.42844 & 0.53675 & 0.5 \\
\hline $\mathrm{H}$ & 0.35395 & 0.52379 & 0.5 \\
\hline $\mathrm{N}$ & 0.96927 & 0.50246 & 0 \\
\hline $\mathrm{N}$ & 0 & 0.5 & 0.06379 \\
\hline $\mathrm{Cu}$ & 0.5 & 1.5 & 1.5 \\
\hline $\mathrm{Cu}$ & 1 & 1.5 & 1 \\
\hline
\end{tabular}

Table S12. Fractional atomic coordinates for the unit cell of COF-2 with the noninterpenetrated tbo topology.

\begin{tabular}{|c|c|c|c|}
\hline \multicolumn{3}{|l|}{ Space group: $P \mathrm{~m}-3$} \\
\hline $\mathrm{a}=\mathrm{b}=\mathrm{c}=95.2459 \AA, \alpha=\beta=\gamma=90^{\circ}$ \\
\hline Atom & $\mathrm{x}$ & $\mathrm{y}$ & $\mathrm{z}$ \\
\hline $\mathrm{C}$ & 0.27643 & 0.72864 & 0.46505 \\
\hline
\end{tabular}




\begin{tabular}{|c|c|c|c|}
\hline $\mathrm{C}$ & 0.27722 & 0.71413 & 0.46428 \\
\hline $\mathrm{C}$ & 0.28441 & 0.7078 & 0.45337 \\
\hline $\mathrm{C}$ & 0.29062 & 0.71585 & 0.44298 \\
\hline $\mathrm{C}$ & 0.29014 & 0.73043 & 0.444 \\
\hline $\mathrm{C}$ & 0.28309 & 0.73676 & 0.45498 \\
\hline $\mathrm{N}$ & 0.29732 & 0.70893 & 0.43159 \\
\hline $\mathrm{C}$ & 0.29868 & 0.71411 & 0.4192 \\
\hline $\mathrm{C}$ & 0.30547 & 0.70617 & 0.408 \\
\hline $\mathrm{C}$ & 0.30429 & 0.71085 & 0.39427 \\
\hline $\mathrm{C}$ & 0.31033 & 0.70339 & 0.3834 \\
\hline $\mathrm{C}$ & 0.31785 & 0.69114 & 0.38605 \\
\hline $\mathrm{C}$ & 0.31915 & 0.68656 & 0.39986 \\
\hline $\mathrm{C}$ & 0.31298 & 0.69396 & 0.41073 \\
\hline $\mathrm{C}$ & 0.32409 & 0.68314 & 0.37439 \\
\hline $\mathrm{C}$ & 0.32968 & 0.69004 & 0.3628 \\
\hline $\mathrm{C}$ & 0.33491 & 0.68255 & 0.35155 \\
\hline $\mathrm{C}$ & 0.33487 & 0.66797 & 0.35168 \\
\hline $\mathrm{C}$ & 0.3296 & 0.66104 & 0.36338 \\
\hline $\mathrm{C}$ & 0.32421 & 0.66853 & 0.37458 \\
\hline $\mathrm{C}$ & 0.77581 & 0.22602 & 0.46508 \\
\hline $\mathrm{C}$ & 0.77022 & 0.21427 & 0.45857 \\
\hline $\mathrm{C}$ & 0.77756 & 0.20758 & 0.448 \\
\hline $\mathrm{C}$ & 0.79055 & 0.21252 & 0.44371 \\
\hline $\mathrm{C}$ & 0.79626 & 0.22423 & 0.45041 \\
\hline $\mathrm{C}$ & 0.7889 & 0.23091 & 0.461 \\
\hline $\mathrm{N}$ & 0.79744 & 0.20532 & 0.43259 \\
\hline $\mathrm{C}$ & 0.80874 & 0.20906 & 0.42623 \\
\hline $\mathrm{C}$ & 0.81439 & 0.20065 & 0.41472 \\
\hline $\mathrm{C}$ & 0.82634 & 0.20518 & 0.40774 \\
\hline $\mathrm{C}$ & 0.83168 & 0.19759 & 0.39659 \\
\hline $\mathrm{C}$ & 0.82517 & 0.18529 & 0.39219 \\
\hline $\mathrm{C}$ & 0.81327 & 0.18068 & 0.3993 \\
\hline $\mathrm{C}$ & 0.80793 & 0.18829 & 0.41044 \\
\hline $\mathrm{C}$ & 0.83061 & 0.17742 & 0.3801 \\
\hline $\mathrm{C}$ & 0.83528 & 0.18447 & 0.3682 \\
\hline $\mathrm{C}$ & 0.84012 & 0.17708 & 0.3567 \\
\hline $\mathrm{C}$ & 0.84042 & 0.1625 & 0.35685 \\
\hline $\mathrm{C}$ & 0.83585 & 0.15545 & 0.36876 \\
\hline $\mathrm{C}$ & 0.83097 & 0.16283 & 0.38027 \\
\hline $\mathrm{C}$ & 0.27521 & 0.22596 & 0.96517 \\
\hline $\mathrm{C}$ & 0.2716 & 0.21242 & 0.96122 \\
\hline $\mathrm{C}$ & 0.27896 & 0.20569 & 0.95069 \\
\hline
\end{tabular}




\begin{tabular}{|c|c|c|c|}
\hline $\mathrm{C}$ & 0.29005 & 0.21229 & 0.94399 \\
\hline $\mathrm{C}$ & 0.29365 & 0.22592 & 0.94797 \\
\hline $\mathrm{C}$ & 0.28629 & 0.23265 & 0.95851 \\
\hline $\mathrm{N}$ & 0.29694 & 0.20498 & 0.93294 \\
\hline $\mathrm{C}$ & 0.30848 & 0.2085 & 0.92689 \\
\hline $\mathrm{C}$ & 0.3141 & 0.20018 & 0.9153 \\
\hline $\mathrm{C}$ & 0.32631 & 0.20451 & 0.90864 \\
\hline $\mathrm{C}$ & 0.33164 & 0.19699 & 0.89743 \\
\hline $\mathrm{C}$ & 0.32487 & 0.18497 & 0.89267 \\
\hline $\mathrm{C}$ & 0.31272 & 0.18057 & 0.89945 \\
\hline $\mathrm{C}$ & 0.30737 & 0.1881 & 0.91064 \\
\hline $\mathrm{C}$ & 0.33032 & 0.17718 & 0.88054 \\
\hline $\mathrm{C}$ & 0.3353 & 0.1843 & 0.86882 \\
\hline $\mathrm{C}$ & 0.34014 & 0.17698 & 0.85726 \\
\hline $\mathrm{C}$ & 0.34014 & 0.16239 & 0.8572 \\
\hline $\mathrm{C}$ & 0.33528 & 0.15527 & 0.86896 \\
\hline $\mathrm{C}$ & 0.3304 & 0.16258 & 0.88051 \\
\hline $\mathrm{C}$ & 0.77574 & 0.72685 & 0.96515 \\
\hline $\mathrm{C}$ & 0.76992 & 0.71529 & 0.95851 \\
\hline $\mathrm{C}$ & 0.77719 & 0.70851 & 0.94794 \\
\hline $\mathrm{C}$ & 0.79032 & 0.71319 & 0.94378 \\
\hline $\mathrm{C}$ & 0.79626 & 0.72469 & 0.95064 \\
\hline $\mathrm{C}$ & 0.78898 & 0.73145 & 0.96122 \\
\hline $\mathrm{N}$ & 0.79713 & 0.70595 & 0.93263 \\
\hline $\mathrm{C}$ & 0.80838 & 0.70972 & 0.92619 \\
\hline $\mathrm{C}$ & 0.81401 & 0.70129 & 0.91469 \\
\hline $\mathrm{C}$ & 0.82595 & 0.70581 & 0.90768 \\
\hline $\mathrm{C}$ & 0.8313 & 0.69818 & 0.89656 \\
\hline $\mathrm{C}$ & 0.82481 & 0.68585 & 0.89222 \\
\hline $\mathrm{C}$ & 0.81292 & 0.68126 & 0.89933 \\
\hline $\mathrm{C}$ & 0.80757 & 0.6889 & 0.91045 \\
\hline $\mathrm{C}$ & 0.83029 & 0.67792 & 0.88019 \\
\hline $\mathrm{C}$ & 0.83507 & 0.68488 & 0.86828 \\
\hline $\mathrm{C}$ & 0.84 & 0.67743 & 0.85686 \\
\hline $\mathrm{C}$ & 0.84029 & 0.66286 & 0.85709 \\
\hline $\mathrm{C}$ & 0.83559 & 0.65588 & 0.869 \\
\hline $\mathrm{C}$ & 0.83064 & 0.66332 & 0.88043 \\
\hline $\mathrm{C}$ & 0.2617 & 0.45986 & 0.75148 \\
\hline $\mathrm{C}$ & 0.26149 & 0.47316 & 0.74597 \\
\hline $\mathrm{N}$ & 0.25433 & 0.48166 & 0.75454 \\
\hline $\mathrm{C}$ & 0.24686 & 0.47316 & 0.76282 \\
\hline $\mathrm{C}$ & 0.25232 & 0.45984 & 0.7622 \\
\hline
\end{tabular}




\begin{tabular}{|c|c|c|c|}
\hline $\mathrm{C}$ & 0.2333 & 0.4761 & 0.76785 \\
\hline $\mathrm{C}$ & 0.2262 & 0.48883 & 0.76563 \\
\hline $\mathrm{C}$ & 0.21471 & 0.50712 & 0.77333 \\
\hline $\mathrm{C}$ & 0.2646 & 0.47611 & 0.73185 \\
\hline $\mathrm{C}$ & 0.26148 & 0.48885 & 0.72514 \\
\hline $\mathrm{C}$ & 0.26739 & 0.49287 & 0.71263 \\
\hline $\mathrm{C}$ & 0.75339 & 0.04017 & 0.2373 \\
\hline $\mathrm{C}$ & 0.74798 & 0.02683 & 0.2368 \\
\hline $\mathrm{N}$ & 0.75572 & 0.01833 & 0.24484 \\
\hline $\mathrm{C}$ & 0.76314 & 0.02683 & 0.25319 \\
\hline $\mathrm{C}$ & 0.76306 & 0.04017 & 0.24775 \\
\hline $\mathrm{C}$ & 0.76673 & 0.02387 & 0.26719 \\
\hline $\mathrm{C}$ & 0.7637 & 0.01116 & 0.27403 \\
\hline $\mathrm{C}$ & 0.77011 & 0.99288 & 0.28629 \\
\hline $\mathrm{C}$ & 0.73429 & 0.02388 & 0.23214 \\
\hline $\mathrm{C}$ & 0.72724 & 0.01116 & 0.23459 \\
\hline $\mathrm{C}$ & 0.71554 & 0.00712 & 0.22723 \\
\hline $\mathrm{N}$ & 0.84517 & 0.15487 & 0.34484 \\
\hline $\mathrm{H}$ & 0.27215 & 0.70776 & 0.47204 \\
\hline $\mathrm{H}$ & 0.28483 & 0.69658 & 0.4528 \\
\hline $\mathrm{H}$ & 0.29524 & 0.73695 & 0.43641 \\
\hline $\mathrm{H}$ & 0.28261 & 0.74798 & 0.45553 \\
\hline $\mathrm{H}$ & 0.29413 & 0.72408 & 0.41668 \\
\hline $\mathrm{H}$ & 0.29849 & 0.72019 & 0.39191 \\
\hline $\mathrm{H}$ & 0.30886 & 0.70709 & 0.3729 \\
\hline $\mathrm{H}$ & 0.32505 & 0.6773 & 0.4023 \\
\hline $\mathrm{H}$ & 0.31414 & 0.69017 & 0.42125 \\
\hline $\mathrm{H}$ & 0.33001 & 0.70127 & 0.36239 \\
\hline $\mathrm{H}$ & 0.33883 & 0.68817 & 0.34263 \\
\hline $\mathrm{H}$ & 0.32949 & 0.64981 & 0.36375 \\
\hline $\mathrm{H}$ & 0.31982 & 0.66286 & 0.38322 \\
\hline $\mathrm{H}$ & 0.76012 & 0.21036 & 0.46162 \\
\hline $\mathrm{H}$ & 0.77306 & 0.19857 & 0.44302 \\
\hline $\mathrm{H}$ & 0.80633 & 0.2283 & 0.44766 \\
\hline $\mathrm{H}$ & 0.79338 & 0.23993 & 0.46601 \\
\hline $\mathrm{H}$ & 0.81405 & 0.21856 & 0.42895 \\
\hline $\mathrm{H}$ & 0.83153 & 0.21466 & 0.41088 \\
\hline $\mathrm{H}$ & 0.84096 & 0.20131 & 0.39144 \\
\hline $\mathrm{H}$ & 0.80792 & 0.17135 & 0.39605 \\
\hline $\mathrm{H}$ & 0.79867 & 0.18454 & 0.4156 \\
\hline $\mathrm{H}$ & 0.83493 & 0.19569 & 0.36768 \\
\hline $\mathrm{H}$ & 0.84344 & 0.18274 & 0.34757 \\
\hline
\end{tabular}




\begin{tabular}{|c|c|c|c|}
\hline $\mathrm{H}$ & 0.8361 & 0.14422 & 0.36915 \\
\hline $\mathrm{H}$ & 0.82761 & 0.15713 & 0.38935 \\
\hline $\mathrm{H}$ & 0.26305 & 0.20713 & 0.96625 \\
\hline $\mathrm{H}$ & 0.27599 & 0.19527 & 0.94768 \\
\hline $\mathrm{H}$ & 0.30189 & 0.23156 & 0.94288 \\
\hline $\mathrm{H}$ & 0.2891 & 0.24315 & 0.96139 \\
\hline $\mathrm{H}$ & 0.31423 & 0.21756 & 0.9302 \\
\hline $\mathrm{H}$ & 0.3317 & 0.21377 & 0.91207 \\
\hline $\mathrm{H}$ & 0.34112 & 0.20054 & 0.89252 \\
\hline $\mathrm{H}$ & 0.30719 & 0.17144 & 0.89591 \\
\hline $\mathrm{H}$ & 0.29792 & 0.1845 & 0.91555 \\
\hline $\mathrm{H}$ & 0.33518 & 0.19553 & 0.86845 \\
\hline $\mathrm{H}$ & 0.34367 & 0.1827 & 0.84825 \\
\hline $\mathrm{H}$ & 0.33531 & 0.14403 & 0.86919 \\
\hline $\mathrm{H}$ & 0.32679 & 0.15682 & 0.88946 \\
\hline $\mathrm{H}$ & 0.75971 & 0.7116 & 0.96145 \\
\hline $\mathrm{H}$ & 0.77252 & 0.69965 & 0.94284 \\
\hline $\mathrm{H}$ & 0.80647 & 0.7285 & 0.94803 \\
\hline $\mathrm{H}$ & 0.79364 & 0.74031 & 0.96634 \\
\hline $\mathrm{H}$ & 0.81364 & 0.71927 & 0.92882 \\
\hline $\mathrm{H}$ & 0.83113 & 0.7153 & 0.91079 \\
\hline $\mathrm{H}$ & 0.84058 & 0.70189 & 0.89141 \\
\hline $\mathrm{H}$ & 0.80759 & 0.6719 & 0.89612 \\
\hline $\mathrm{H}$ & 0.79832 & 0.68516 & 0.91563 \\
\hline $\mathrm{H}$ & 0.83473 & 0.6961 & 0.86768 \\
\hline $\mathrm{H}$ & 0.8434 & 0.68303 & 0.84772 \\
\hline $\mathrm{H}$ & 0.83582 & 0.64465 & 0.86944 \\
\hline $\mathrm{H}$ & 0.82722 & 0.65767 & 0.88952 \\
\hline $\mathrm{H}$ & 0.26617 & 0.45063 & 0.74688 \\
\hline $\mathrm{H}$ & 0.24833 & 0.45058 & 0.76716 \\
\hline $\mathrm{H}$ & 0.20852 & 0.5135 & 0.7802 \\
\hline $\mathrm{H}$ & 0.27317 & 0.48645 & 0.70544 \\
\hline $\mathrm{H}$ & 0.74919 & 0.04944 & 0.23253 \\
\hline $\mathrm{H}$ & 0.76751 & 0.04943 & 0.25231 \\
\hline $\mathrm{H}$ & 0.77629 & 0.9865 & 0.29318 \\
\hline $\mathrm{H}$ & 0.70915 & 0.0135 & 0.22054 \\
\hline $\mathrm{N}$ & 0.75909 & 0.23162 & 0.5 \\
\hline $\mathrm{N}$ & 0.73142 & 0.25578 & 0.5 \\
\hline $\mathrm{N}$ & 0.26795 & 0.75777 & 0 \\
\hline $\mathrm{N}$ & 0.24099 & 0.73284 & 0 \\
\hline $\mathrm{N}$ & 0.33978 & 0.66022 & 0.33978 \\
\hline $\mathrm{Cu}$ & 1.24967 & 1.2495 & 1 \\
\hline
\end{tabular}


Table S13. Fractional atomic coordinates for the unit cell of COF-2 with the interpenetrated pto topology.

\begin{tabular}{|c|c|c|c|}
\hline \multicolumn{4}{|c|}{ Space group: $P-421 \mathrm{c}$} \\
\hline \multicolumn{4}{|c|}{$\mathrm{a}=\mathrm{b}=57.4067 \AA, \mathrm{c}=52.7041 \AA, \alpha=\beta=\gamma=90^{\circ}$} \\
\hline Atom & $\mathrm{x}$ & $\mathrm{y}$ & $\mathrm{Z}$ \\
\hline $\mathrm{C}$ & 0.98939 & 0.55067 & 0.12256 \\
\hline $\mathrm{N}$ & 1.00553 & 0.53334 & 0.12336 \\
\hline $\mathrm{C}$ & 0.02642 & 0.5444 & 0.12405 \\
\hline $\mathrm{C}$ & 0.02354 & 0.56818 & 0.12053 \\
\hline $\mathrm{C}$ & 1.0001 & 0.57216 & 0.11959 \\
\hline $\mathrm{C}$ & 0.048 & 0.53411 & 0.12995 \\
\hline $\mathrm{C}$ & 0.06929 & 0.54888 & 0.12806 \\
\hline $\mathrm{C}$ & 0.07596 & 0.56346 & 0.14816 \\
\hline $\mathrm{C}$ & 0.09585 & 0.57744 & 0.14598 \\
\hline $\mathrm{C}$ & 0.10946 & 0.57692 & 0.12388 \\
\hline $\mathrm{C}$ & 0.10263 & 0.56237 & 0.10366 \\
\hline $\mathrm{C}$ & 0.08268 & 0.54852 & 0.10577 \\
\hline $\mathrm{N}$ & 0.12904 & 0.5925 & 0.12217 \\
\hline $\mathrm{C}$ & 0.14589 & 0.59154 & 0.10567 \\
\hline $\mathrm{C}$ & 0.16377 & 0.61008 & 0.10465 \\
\hline $\mathrm{C}$ & 0.17985 & 0.61046 & 0.08457 \\
\hline $\mathrm{C}$ & 0.19548 & 0.62904 & 0.08194 \\
\hline $\mathrm{C}$ & 0.19551 & 0.64747 & 0.09954 \\
\hline $\mathrm{C}$ & 0.18004 & 0.64653 & 0.12024 \\
\hline $\mathrm{C}$ & 0.16429 & 0.62809 & 0.12273 \\
\hline $\mathrm{C}$ & 0.21081 & 0.66811 & 0.09582 \\
\hline $\mathrm{C}$ & 0.21381 & 0.67789 & 0.07152 \\
\hline $\mathrm{C}$ & 0.22691 & 0.69827 & 0.06829 \\
\hline $\mathrm{C}$ & 0.23704 & 0.70933 & 0.08933 \\
\hline $\mathrm{C}$ & 0.23463 & 0.69925 & 0.11344 \\
\hline $\mathrm{C}$ & 0.22164 & 0.67888 & 0.11669 \\
\hline $\mathrm{C}$ & 0.01033 & 0.55074 & 0.6358 \\
\hline $\mathrm{N}$ & -0.0059 & 0.53351 & 0.63607 \\
\hline $\mathrm{C}$ & 0.97328 & 0.54475 & 0.63418 \\
\hline $\mathrm{C}$ & 0.97631 & 0.56862 & 0.63669 \\
\hline $\mathrm{C}$ & -0.00025 & 0.57238 & 0.63785 \\
\hline $\mathrm{C}$ & 0.95193 & 0.53461 & 0.62695 \\
\hline
\end{tabular}




\begin{tabular}{|c|c|c|c|}
\hline $\mathrm{C}$ & 0.93152 & 0.55026 & 0.62224 \\
\hline $\mathrm{C}$ & 0.92948 & 0.56167 & 0.59881 \\
\hline $\mathrm{C}$ & 0.91108 & 0.57711 & 0.59432 \\
\hline $\mathrm{C}$ & 0.89433 & 0.58154 & 0.61307 \\
\hline $\mathrm{C}$ & 0.89608 & 0.56977 & 0.63653 \\
\hline $\mathrm{C}$ & 0.91463 & 0.55432 & 0.64106 \\
\hline $\mathrm{N}$ & 0.87692 & 0.59899 & 0.60777 \\
\hline $\mathrm{C}$ & 0.85822 & 0.60378 & 0.62074 \\
\hline $\mathrm{C}$ & 0.84246 & 0.62294 & 0.61263 \\
\hline $\mathrm{C}$ & 0.82131 & 0.62671 & 0.62545 \\
\hline $\mathrm{C}$ & 0.80629 & 0.64472 & 0.61798 \\
\hline $\mathrm{C}$ & 0.81211 & 0.6594 & 0.59753 \\
\hline $\mathrm{C}$ & 0.83339 & 0.65577 & 0.58491 \\
\hline $\mathrm{C}$ & 0.84837 & 0.63776 & 0.59233 \\
\hline $\mathrm{C}$ & 0.79621 & 0.67854 & 0.58947 \\
\hline $\mathrm{C}$ & 0.78442 & 0.69212 & 0.60763 \\
\hline $\mathrm{C}$ & 0.76972 & 0.71025 & 0.60004 \\
\hline $\mathrm{C}$ & 0.76623 & 0.71501 & 0.57418 \\
\hline $\mathrm{C}$ & 0.77811 & 0.70171 & 0.55599 \\
\hline $\mathrm{C}$ & 0.79283 & 0.68353 & 0.56354 \\
\hline $\mathrm{C}$ & 0.23203 & 0.98428 & 0.38046 \\
\hline $\mathrm{N}$ & 0.23042 & 1.00211 & 0.36351 \\
\hline $\mathrm{C}$ & 0.2311 & 0.02176 & 0.37803 \\
\hline $\mathrm{C}$ & 0.23143 & 0.01627 & 0.40359 \\
\hline $\mathrm{C}$ & 0.23226 & 0.99265 & 0.40516 \\
\hline $\mathrm{C}$ & 0.2336 & 0.04467 & 0.36918 \\
\hline $\mathrm{C}$ & 0.23396 & 0.06417 & 0.3881 \\
\hline $\mathrm{C}$ & 0.25431 & 0.06925 & 0.40184 \\
\hline $\mathrm{C}$ & 0.25447 & 0.08734 & 0.41971 \\
\hline $\mathrm{C}$ & 0.23442 & 0.10071 & 0.42419 \\
\hline $\mathrm{C}$ & 0.21401 & 0.09563 & 0.41035 \\
\hline $\mathrm{C}$ & 0.21381 & 0.07744 & 0.39257 \\
\hline $\mathrm{N}$ & 0.23588 & 0.11973 & 0.44202 \\
\hline $\mathrm{C}$ & 0.21853 & 0.13073 & 0.45258 \\
\hline $\mathrm{C}$ & 0.22267 & 0.15074 & 0.46984 \\
\hline $\mathrm{C}$ & 0.20369 & 0.1623 & 0.48106 \\
\hline $\mathrm{C}$ & 0.20726 & 0.18165 & 0.49683 \\
\hline $\mathrm{C}$ & 0.2299 & 0.19005 & 0.50143 \\
\hline $\mathrm{C}$ & 0.24888 & 0.17811 & 0.49062 \\
\hline $\mathrm{C}$ & 0.24532 & 0.15871 & 0.47503 \\
\hline $\mathrm{C}$ & 0.23391 & 0.21116 & 0.51732 \\
\hline $\mathrm{C}$ & 0.22055 & 0.21532 & 0.53929 \\
\hline
\end{tabular}




\begin{tabular}{|c|c|c|c|}
\hline $\mathrm{C}$ & 0.2254 & 0.23447 & 0.5549 \\
\hline $\mathrm{C}$ & 0.24381 & 0.24961 & 0.54897 \\
\hline $\mathrm{C}$ & 0.2567 & 0.24595 & 0.52675 \\
\hline $\mathrm{C}$ & 0.25169 & 0.22703 & 0.51097 \\
\hline $\mathrm{C}$ & 0.22395 & 0.01605 & 0.27268 \\
\hline $\mathrm{N}$ & 0.22481 & -0.00191 & 0.28953 \\
\hline $\mathrm{C}$ & 0.2231 & 0.97854 & 0.27504 \\
\hline $\mathrm{C}$ & 0.21932 & 0.98423 & 0.24973 \\
\hline $\mathrm{C}$ & 0.2199 & 0.00794 & 0.24828 \\
\hline $\mathrm{C}$ & 0.22681 & 0.95554 & 0.2834 \\
\hline $\mathrm{C}$ & 0.22588 & 0.93611 & 0.26453 \\
\hline $\mathrm{C}$ & 0.24525 & 0.93145 & 0.24914 \\
\hline $\mathrm{C}$ & 0.24492 & 0.91273 & 0.23209 \\
\hline $\mathrm{C}$ & 0.2254 & 0.8981 & 0.23016 \\
\hline $\mathrm{C}$ & 0.20582 & 0.90284 & 0.24558 \\
\hline $\mathrm{C}$ & 0.20611 & 0.92175 & 0.26257 \\
\hline $\mathrm{N}$ & 0.2263 & 0.87913 & 0.21211 \\
\hline $\mathrm{C}$ & 0.21249 & 0.86112 & 0.21058 \\
\hline $\mathrm{C}$ & 0.21641 & 0.84269 & 0.19105 \\
\hline $\mathrm{C}$ & 0.20383 & 0.82165 & 0.19209 \\
\hline $\mathrm{C}$ & 0.20816 & 0.8037 & 0.17455 \\
\hline $\mathrm{C}$ & 0.22518 & 0.80638 & 0.15551 \\
\hline $\mathrm{C}$ & 0.23718 & 0.82773 & 0.15401 \\
\hline $\mathrm{C}$ & 0.2329 & 0.84562 & 0.17153 \\
\hline $\mathrm{C}$ & 0.23102 & 0.78691 & 0.13774 \\
\hline $\mathrm{C}$ & 0.21352 & 0.77265 & 0.12722 \\
\hline $\mathrm{C}$ & 0.21927 & 0.75466 & 0.11032 \\
\hline $\mathrm{C}$ & 0.24262 & 0.7504 & 0.10368 \\
\hline $\mathrm{C}$ & 0.26011 & 0.76443 & 0.11433 \\
\hline $\mathrm{C}$ & 0.2544 & 0.7824 & 0.13117 \\
\hline $\mathrm{C}$ & 0.55 & 0.26818 & 0.80879 \\
\hline $\mathrm{N}$ & 0.53457 & 0.26573 & 0.82812 \\
\hline $\mathrm{C}$ & 0.54773 & 0.26416 & -0.15054 \\
\hline $\mathrm{C}$ & 0.57123 & 0.26367 & -0.15664 \\
\hline $\mathrm{C}$ & 0.57266 & 0.26613 & 0.81765 \\
\hline $\mathrm{C}$ & 0.53935 & 0.26481 & -0.12552 \\
\hline $\mathrm{C}$ & 0.55656 & 0.26315 & -0.10432 \\
\hline $\mathrm{C}$ & 0.56145 & 0.2827 & -0.08923 \\
\hline $\mathrm{C}$ & 0.5781 & 0.28136 & -0.06986 \\
\hline $\mathrm{C}$ & 0.59063 & 0.26072 & -0.06575 \\
\hline $\mathrm{C}$ & 0.58527 & 0.24089 & -0.08042 \\
\hline $\mathrm{C}$ & 0.5683 & 0.24214 & -0.09951 \\
\hline
\end{tabular}




\begin{tabular}{|c|c|c|c|}
\hline $\mathrm{N}$ & 0.60847 & 0.26053 & -0.04652 \\
\hline $\mathrm{C}$ & 0.62711 & 0.24759 & -0.04679 \\
\hline $\mathrm{C}$ & 0.64472 & 0.24939 & -0.02627 \\
\hline $\mathrm{C}$ & 0.66711 & 0.24006 & -0.02985 \\
\hline $\mathrm{C}$ & 0.68422 & 0.24247 & -0.01105 \\
\hline $\mathrm{C}$ & 0.67931 & 0.25414 & 0.01186 \\
\hline $\mathrm{C}$ & 0.65665 & 0.26288 & 0.01562 \\
\hline $\mathrm{C}$ & 0.6396 & 0.26065 & -0.00323 \\
\hline $\mathrm{C}$ & 0.69784 & 0.25771 & 0.03142 \\
\hline $\mathrm{C}$ & 0.71524 & 0.2408 & 0.03543 \\
\hline $\mathrm{C}$ & 0.73295 & 0.24453 & 0.05322 \\
\hline $\mathrm{C}$ & 0.73372 & 0.2652 & 0.06745 \\
\hline $\mathrm{C}$ & 0.71645 & 0.28214 & 0.06353 \\
\hline $\mathrm{C}$ & 0.69854 & 0.2783 & 0.046 \\
\hline $\mathrm{C}$ & 0.44994 & 0.2647 & -0.15667 \\
\hline $\mathrm{N}$ & 0.46549 & 0.26548 & -0.17612 \\
\hline $\mathrm{C}$ & 0.45225 & 0.26684 & 0.80262 \\
\hline $\mathrm{C}$ & 0.4288 & 0.26458 & 0.80847 \\
\hline $\mathrm{C}$ & 0.42733 & 0.26328 & -0.16577 \\
\hline $\mathrm{C}$ & 0.46044 & 0.27145 & 0.77807 \\
\hline $\mathrm{C}$ & 0.44321 & 0.27205 & 0.7569 \\
\hline $\mathrm{C}$ & 0.42909 & 0.29171 & 0.75313 \\
\hline $\mathrm{C}$ & 0.41293 & 0.29218 & 0.73323 \\
\hline $\mathrm{C}$ & 0.41083 & 0.27324 & 0.71655 \\
\hline $\mathrm{C}$ & 0.42473 & 0.25332 & 0.72069 \\
\hline $\mathrm{C}$ & 0.44079 & 0.2528 & 0.7407 \\
\hline $\mathrm{N}$ & 0.39386 & 0.27463 & 0.69644 \\
\hline $\mathrm{C}$ & 0.39318 & 0.26176 & 0.67608 \\
\hline $\mathrm{C}$ & 0.37401 & 0.26421 & 0.65738 \\
\hline $\mathrm{C}$ & 0.37305 & 0.2492 & 0.63632 \\
\hline $\mathrm{C}$ & 0.35435 & 0.25028 & 0.61925 \\
\hline $\mathrm{C}$ & 0.3363 & 0.26652 & 0.62281 \\
\hline $\mathrm{C}$ & 0.33765 & 0.28201 & 0.64345 \\
\hline $\mathrm{C}$ & 0.35627 & 0.28088 & 0.66055 \\
\hline $\mathrm{C}$ & 0.31556 & 0.26675 & 0.60585 \\
\hline $\mathrm{C}$ & 0.3048 & 0.24577 & 0.59849 \\
\hline $\mathrm{C}$ & 0.28393 & 0.24603 & 0.58475 \\
\hline $\mathrm{C}$ & 0.27338 & 0.26727 & 0.57807 \\
\hline $\mathrm{C}$ & 0.28475 & 0.2882 & 0.58431 \\
\hline $\mathrm{C}$ & 0.30552 & 0.28795 & 0.59815 \\
\hline $\mathrm{N}$ & 0.25054 & 0.26768 & 0.56645 \\
\hline $\mathrm{N}$ & 0.75144 & 0.26845 & 0.0866 \\
\hline
\end{tabular}




\begin{tabular}{|c|c|c|c|}
\hline $\mathrm{H}$ & 0.03688 & 0.58155 & 0.12058 \\
\hline $\mathrm{H}$ & 0.99198 & 0.5892 & 0.11903 \\
\hline $\mathrm{H}$ & 0.0656 & 0.56427 & 0.16533 \\
\hline $\mathrm{H}$ & 0.10064 & 0.58886 & 0.16149 \\
\hline $\mathrm{H}$ & 0.11215 & 0.562 & 0.08598 \\
\hline $\mathrm{H}$ & 0.0775 & 0.53763 & 0.08994 \\
\hline $\mathrm{H}$ & 0.14674 & 0.57761 & 0.09187 \\
\hline $\mathrm{H}$ & 0.17992 & 0.59664 & 0.07053 \\
\hline $\mathrm{H}$ & 0.2074 & 0.62907 & 0.06601 \\
\hline $\mathrm{H}$ & 0.17949 & 0.66048 & 0.13405 \\
\hline $\mathrm{H}$ & 0.15218 & 0.6282 & 0.13851 \\
\hline $\mathrm{H}$ & 0.20533 & 0.67025 & 0.05515 \\
\hline $\mathrm{H}$ & 0.2284 & 0.7059 & 0.04957 \\
\hline $\mathrm{H}$ & 0.24259 & 0.70745 & 0.1298 \\
\hline $\mathrm{H}$ & 0.21991 & 0.67172 & 0.1356 \\
\hline $\mathrm{H}$ & 0.96318 & 0.58215 & 0.63545 \\
\hline $\mathrm{H}$ & 0.00809 & 0.58931 & 0.63755 \\
\hline $\mathrm{H}$ & 0.94244 & 0.55893 & 0.58415 \\
\hline $\mathrm{H}$ & 0.91002 & 0.58594 & 0.57618 \\
\hline $\mathrm{H}$ & 0.88365 & 0.57275 & 0.6516 \\
\hline $\mathrm{H}$ & 0.91606 & 0.54577 & 0.65933 \\
\hline $\mathrm{H}$ & 0.85359 & 0.59362 & 0.63729 \\
\hline $\mathrm{H}$ & 0.81637 & 0.61569 & 0.64127 \\
\hline $\mathrm{H}$ & 0.78995 & 0.64695 & 0.62798 \\
\hline $\mathrm{H}$ & 0.83863 & 0.66709 & 0.56947 \\
\hline $\mathrm{H}$ & 0.8647 & 0.63561 & 0.5823 \\
\hline $\mathrm{H}$ & 0.78662 & 0.68884 & 0.62773 \\
\hline $\mathrm{H}$ & 0.76086 & 0.72055 & 0.61433 \\
\hline $\mathrm{H}$ & 0.77539 & 0.70496 & 0.53595 \\
\hline $\mathrm{H}$ & 0.80107 & 0.67297 & 0.54908 \\
\hline $\mathrm{H}$ & 0.23272 & 0.02814 & 0.41951 \\
\hline $\mathrm{H}$ & 0.23446 & 0.98291 & 0.42261 \\
\hline $\mathrm{H}$ & 0.26997 & 0.05905 & 0.3989 \\
\hline $\mathrm{H}$ & 0.27024 & 0.09084 & 0.43032 \\
\hline $\mathrm{H}$ & 0.1983 & 0.10575 & 0.41285 \\
\hline $\mathrm{H}$ & 0.19794 & 0.07371 & 0.3822 \\
\hline $\mathrm{H}$ & 0.20074 & 0.12541 & 0.44905 \\
\hline $\mathrm{H}$ & 0.18603 & 0.1565 & 0.47743 \\
\hline $\mathrm{H}$ & 0.19223 & 0.19048 & 0.50476 \\
\hline $\mathrm{H}$ & 0.26658 & 0.18368 & 0.49434 \\
\hline $\mathrm{H}$ & 0.26034 & 0.15002 & 0.46697 \\
\hline $\mathrm{H}$ & 0.20686 & 0.20338 & 0.54487 \\
\hline
\end{tabular}




\begin{tabular}{|c|c|c|c|}
\hline $\mathrm{H}$ & 0.21539 & 0.23703 & 0.5721 \\
\hline $\mathrm{H}$ & 0.27081 & 0.25765 & 0.52185 \\
\hline $\mathrm{H}$ & 0.26189 & 0.22474 & 0.49386 \\
\hline $\mathrm{H}$ & 0.21773 & 0.97256 & 0.23363 \\
\hline $\mathrm{H}$ & 0.21892 & 0.01808 & 0.23094 \\
\hline $\mathrm{H}$ & 0.26069 & 0.94224 & 0.25053 \\
\hline $\mathrm{H}$ & 0.26019 & 0.90936 & 0.22057 \\
\hline $\mathrm{H}$ & 0.19028 & 0.89222 & 0.24461 \\
\hline $\mathrm{H}$ & 0.19106 & 0.92512 & 0.27444 \\
\hline $\mathrm{H}$ & 0.19865 & 0.85869 & 0.22428 \\
\hline $\mathrm{H}$ & 0.19093 & 0.81889 & 0.20681 \\
\hline $\mathrm{H}$ & 0.19867 & 0.78745 & 0.17634 \\
\hline $\mathrm{H}$ & 0.24995 & 0.8306 & 0.1392 \\
\hline $\mathrm{H}$ & 0.24262 & 0.86171 & 0.16983 \\
\hline $\mathrm{H}$ & 0.19534 & 0.77556 & 0.13171 \\
\hline $\mathrm{H}$ & 0.20551 & 0.74411 & 0.10218 \\
\hline $\mathrm{H}$ & 0.27826 & 0.76123 & 0.10984 \\
\hline $\mathrm{H}$ & 0.26837 & 0.79257 & 0.13941 \\
\hline $\mathrm{H}$ & 0.586 & 0.26263 & -0.14386 \\
\hline $\mathrm{H}$ & 0.58868 & 0.26727 & 0.80688 \\
\hline $\mathrm{H}$ & 0.55247 & 0.29901 & -0.09262 \\
\hline $\mathrm{H}$ & 0.58171 & 0.29668 & -0.0585 \\
\hline $\mathrm{H}$ & 0.59403 & 0.22446 & -0.07715 \\
\hline $\mathrm{H}$ & 0.56447 & 0.22684 & -0.11082 \\
\hline $\mathrm{H}$ & 0.63069 & 0.23634 & -0.06286 \\
\hline $\mathrm{H}$ & 0.67159 & 0.23138 & -0.04746 \\
\hline $\mathrm{H}$ & 0.70149 & 0.23573 & -0.01476 \\
\hline $\mathrm{H}$ & 0.65199 & 0.27138 & 0.03325 \\
\hline $\mathrm{H}$ & 0.62242 & 0.26777 & 0.00026 \\
\hline $\mathrm{H}$ & 0.71499 & 0.22445 & 0.02518 \\
\hline $\mathrm{H}$ & 0.7461 & 0.23124 & 0.05599 \\
\hline $\mathrm{H}$ & 0.71657 & 0.29818 & 0.07438 \\
\hline $\mathrm{H}$ & 0.68544 & 0.29169 & 0.04363 \\
\hline $\mathrm{H}$ & 0.41409 & 0.26482 & 0.79562 \\
\hline $\mathrm{H}$ & 0.41129 & 0.26231 & -0.15498 \\
\hline $\mathrm{H}$ & 0.43052 & 0.3066 & 0.7657 \\
\hline $\mathrm{H}$ & 0.40205 & 0.30743 & 0.73068 \\
\hline $\mathrm{H}$ & 0.42301 & 0.23798 & 0.70894 \\
\hline $\mathrm{H}$ & 0.45135 & 0.23738 & 0.74367 \\
\hline $\mathrm{H}$ & 0.40657 & 0.2489 & 0.67248 \\
\hline $\mathrm{H}$ & 0.38665 & 0.23641 & 0.63325 \\
\hline $\mathrm{H}$ & 0.35398 & 0.23836 & 0.60331 \\
\hline
\end{tabular}




\begin{tabular}{|r|r|r|r|}
\hline $\mathrm{H}$ & 0.3239 & 0.29456 & 0.64683 \\
\hline $\mathrm{H}$ & 0.35658 & 0.29285 & 0.67644 \\
\hline $\mathrm{H}$ & 0.31196 & 0.22914 & 0.6043 \\
\hline $\mathrm{H}$ & 0.27553 & 0.22964 & 0.58025 \\
\hline $\mathrm{H}$ & 0.27721 & 0.30479 & 0.57891 \\
\hline $\mathrm{H}$ & 0.31358 & 0.3044 & 0.60314 \\
\hline $\mathrm{Cu}$ & 0.49992 & 1.7344 & 0.826 \\
\hline $\mathrm{Cu}$ & 1 & 1.5 & 0.63038 \\
\hline
\end{tabular}

Table S14. Fractional atomic coordinates for the unit cell of COF-2 with the interpenetrated ffc topology.

\begin{tabular}{|c|c|c|c|}
\hline \multicolumn{4}{|c|}{ Space group:I4/m } \\
\hline \multicolumn{4}{|c|}{$\mathrm{a}=\mathrm{b}=65.666 \AA, \mathrm{c}=31.8663 \AA, \alpha=\beta=\gamma=90^{\circ}$} \\
\hline Atom & $\mathrm{x}$ & $\mathrm{y}$ & $\mathrm{Z}$ \\
\hline $\mathrm{C}$ & 0.91348 & 0.99593 & 0.53905 \\
\hline $\mathrm{C}$ & 0.89189 & 0.99493 & 0.53902 \\
\hline $\mathrm{C}$ & 0.81422 & 0.00692 & 0.46122 \\
\hline $\mathrm{C}$ & 0.55338 & 0.99688 & 0.1117 \\
\hline $\mathrm{C}$ & 0.5631 & 0.01497 & 0.12595 \\
\hline $\mathrm{C}$ & 0.5775 & 0.01413 & 0.15944 \\
\hline $\mathrm{C}$ & 0.58223 & 0.9953 & 0.17941 \\
\hline $\mathrm{C}$ & 0.57282 & 0.97705 & 0.16421 \\
\hline $\mathrm{C}$ & 0.55845 & 0.9779 & 0.13067 \\
\hline $\mathrm{N}$ & 0.59661 & 0.99547 & 0.21472 \\
\hline $\mathrm{C}$ & 0.59931 & 0.98077 & 0.24267 \\
\hline $\mathrm{C}$ & 0.61424 & 0.98312 & 0.2783 \\
\hline $\mathrm{C}$ & 0.61568 & 0.96759 & 0.30964 \\
\hline $\mathrm{C}$ & 0.62918 & 0.96974 & 0.34446 \\
\hline $\mathrm{C}$ & 0.64156 & 0.98748 & 0.34861 \\
\hline $\mathrm{C}$ & 0.6403 & 0.00291 & 0.31684 \\
\hline $\mathrm{C}$ & 0.62678 & 0.00077 & 0.28203 \\
\hline $\mathrm{C}$ & 0.65531 & 0.99008 & 0.38654 \\
\hline $\mathrm{C}$ & 0.66635 & 0.97316 & 0.40333 \\
\hline $\mathrm{C}$ & 0.67852 & 0.97545 & 0.44019 \\
\hline $\mathrm{C}$ & 0.67989 & 0.99466 & 0.46074 \\
\hline $\mathrm{C}$ & 0.66923 & 0.01165 & 0.44386 \\
\hline $\mathrm{C}$ & 0.65703 & 0.0094 & 0.40704 \\
\hline $\mathrm{C}$ & 0.79268 & 0.00516 & 0.53892 \\
\hline $\mathrm{C}$ & 0.74641 & 0.0014 & 0.53881 \\
\hline
\end{tabular}




\begin{tabular}{|c|c|c|c|}
\hline $\mathrm{C}$ & 0.72489 & -0.00042 & 0.53875 \\
\hline $\mathrm{C}$ & 0.00095 & 0.5166 & 0.90943 \\
\hline $\mathrm{C}$ & 0.0024 & 0.54345 & 0.03463 \\
\hline $\mathrm{C}$ & 0.00363 & 0.56393 & 0.02191 \\
\hline $\mathrm{C}$ & 0.51049 & 0.99941 & 0.86668 \\
\hline $\mathrm{C}$ & 0.46273 & 0.00212 & 0.07774 \\
\hline $\mathrm{H}$ & 0.92172 & 0.99629 & 0.56939 \\
\hline $\mathrm{H}$ & 0.8836 & 0.99451 & 0.56928 \\
\hline $\mathrm{H}$ & 0.82246 & 0.00761 & 0.43089 \\
\hline $\mathrm{H}$ & 0.55933 & 0.02973 & 0.11147 \\
\hline $\mathrm{H}$ & 0.58473 & 0.02827 & 0.17046 \\
\hline $\mathrm{H}$ & 0.5765 & 0.96211 & 0.17772 \\
\hline $\mathrm{H}$ & 0.55111 & 0.96381 & 0.11969 \\
\hline $\mathrm{H}$ & 0.59003 & 0.96688 & 0.24159 \\
\hline $\mathrm{H}$ & 0.60613 & 0.95388 & 0.30751 \\
\hline $\mathrm{H}$ & 0.62956 & 0.95771 & 0.36868 \\
\hline $\mathrm{H}$ & 0.64984 & 0.01663 & 0.31882 \\
\hline $\mathrm{H}$ & 0.62607 & 0.01297 & 0.25823 \\
\hline $\mathrm{H}$ & 0.66557 & 0.95817 & 0.38797 \\
\hline $\mathrm{H}$ & 0.68672 & 0.96225 & 0.4531 \\
\hline $\mathrm{H}$ & 0.67018 & 0.02655 & 0.45957 \\
\hline $\mathrm{H}$ & 0.64853 & 0.02266 & 0.39527 \\
\hline $\mathrm{H}$ & 0.78552 & 0.00458 & 0.57013 \\
\hline $\mathrm{H}$ & 0.75358 & 0.00207 & 0.57001 \\
\hline $\mathrm{H}$ & 0.71702 & -0.00095 & 0.56946 \\
\hline $\mathrm{H}$ & 0.0045 & 0.57752 & 0.04213 \\
\hline $\mathrm{H}$ & 0.52015 & 0.9989 & 0.83826 \\
\hline $\mathrm{C}$ & 0.99752 & 0.05273 & 0.5 \\
\hline $\mathrm{C}$ & 0.97902 & 0.04157 & 0.5 \\
\hline $\mathrm{C}$ & 0.95974 & 0.05086 & 0.5 \\
\hline $\mathrm{C}$ & 0.92435 & 0.99645 & 0.5 \\
\hline $\mathrm{C}$ & 0.88105 & 0.9945 & 0.5 \\
\hline $\mathrm{N}$ & 0.85901 & 0.99329 & 0.5 \\
\hline $\mathrm{C}$ & 0.78124 & 0.00423 & 0.5 \\
\hline $\mathrm{C}$ & 0.75785 & 0.00232 & 0.5 \\
\hline $\mathrm{C}$ & 0.71376 & -0.00149 & 0.5 \\
\hline $\mathrm{C}$ & 0.95664 & 0.01698 & 0.5 \\
\hline $\mathrm{C}$ & 0.94559 & 0.03531 & 0.5 \\
\hline $\mathrm{N}$ & 0.97727 & 0.02069 & 0.5 \\
\hline $\mathrm{N}$ & 0.69141 & -0.00333 & 0.5 \\
\hline $\mathrm{C}$ & 0.84757 & 0.00972 & 0.5 \\
\hline $\mathrm{C}$ & 0.82503 & 0.00777 & 0.5 \\
\hline
\end{tabular}




\begin{tabular}{|r|r|r|r|}
\hline $\mathrm{H}$ & 0.95619 & 0.06721 & 0.5 \\
\hline $\mathrm{H}$ & 0.92898 & 0.03732 & 0.5 \\
\hline $\mathrm{H}$ & 0.85446 & 0.02499 & 0.5 \\
\hline $\mathrm{N}$ & 0.46929 & 0.00165 & 0 \\
\hline $\mathrm{N}$ & 0.5 & 0 & 0.06398 \\
\hline $\mathrm{Cu}$ & 0.5 & 0.5 & 1 \\
\hline $\mathrm{Cu}$ & 1 & 0.5 & 0.5 \\
\hline
\end{tabular}

Table S15. Fractional atomic coordinates for the unit cell of COF-2 with the interpenetrated fjh topology.

\begin{tabular}{|c|c|c|c|}
\hline \multicolumn{4}{|c|}{ Space group: $P$ cca } \\
\hline \multicolumn{4}{|c|}{$\mathrm{a}=81.7429 \AA, \mathrm{b}=81.704 \quad \AA, \mathrm{c}=53.2234 \AA, \alpha=\beta=\gamma=90^{\circ}$} \\
\hline Atom & $\mathrm{x}$ & $\mathrm{y}$ & $\mathrm{z}$ \\
\hline $\mathrm{C}$ & 0.36437 & -0.13462 & 0.57209 \\
\hline $\mathrm{C}$ & 0.37977 & -0.12033 & 0.54625 \\
\hline $\mathrm{C}$ & 0.39842 & -0.09953 & 0.64297 \\
\hline $\mathrm{C}$ & 0.40925 & -0.08761 & 0.63483 \\
\hline $\mathrm{C}$ & 0.39427 & -0.10397 & 0.57766 \\
\hline $\mathrm{C}$ & 0.4035 & -0.09543 & 0.55743 \\
\hline $\mathrm{C}$ & 0.39685 & -0.08166 & 0.54543 \\
\hline $\mathrm{C}$ & 0.40598 & -0.07318 & 0.52738 \\
\hline $\mathrm{C}$ & 0.4219 & -0.07817 & 0.52134 \\
\hline $\mathrm{C}$ & 0.42836 & -0.09228 & 0.53291 \\
\hline $\mathrm{C}$ & 0.41923 & -0.10077 & 0.55087 \\
\hline $\mathrm{N}$ & 0.431 & -0.0688 & 0.50326 \\
\hline $\mathrm{C}$ & 0.44685 & -0.06865 & 0.50124 \\
\hline $\mathrm{C}$ & 0.45509 & -0.05867 & 0.48192 \\
\hline $\mathrm{C}$ & 0.47223 & -0.0585 & 0.48097 \\
\hline $\mathrm{C}$ & 0.48039 & -0.04917 & 0.46291 \\
\hline $\mathrm{C}$ & 0.47157 & -0.03978 & 0.44541 \\
\hline $\mathrm{C}$ & 0.45437 & -0.03999 & 0.44635 \\
\hline $\mathrm{C}$ & 0.44621 & -0.04934 & 0.46443 \\
\hline $\mathrm{C}$ & 0.48031 & -0.02981 & 0.42632 \\
\hline $\mathrm{C}$ & 0.49475 & -0.02142 & 0.43268 \\
\hline $\mathrm{C}$ & 0.50317 & -0.0123 & 0.41467 \\
\hline $\mathrm{C}$ & 0.49735 & -0.01134 & 0.38991 \\
\hline $\mathrm{C}$ & 0.48286 & -0.01959 & 0.38353 \\
\hline $\mathrm{C}$ & 0.47442 & -0.02873 & 0.40155 \\
\hline $\mathrm{C}$ & 0.86333 & 0.37026 & 1.08296 \\
\hline
\end{tabular}




\begin{tabular}{|c|c|c|c|}
\hline $\mathrm{C}$ & 0.87812 & 0.38632 & 1.05873 \\
\hline $\mathrm{C}$ & 0.89565 & 0.40396 & 0.15751 \\
\hline $\mathrm{C}$ & 0.90615 & 0.41649 & 0.15061 \\
\hline $\mathrm{C}$ & 0.89168 & 0.40212 & 1.09183 \\
\hline $\mathrm{C}$ & 0.90081 & 0.41176 & 1.07266 \\
\hline $\mathrm{C}$ & 0.8941 & 0.42611 & 1.06242 \\
\hline $\mathrm{C}$ & 0.90339 & 0.43587 & 1.04617 \\
\hline $\mathrm{C}$ & 0.9196 & 0.43172 & 1.04051 \\
\hline $\mathrm{C}$ & 0.92614 & 0.41708 & 1.05018 \\
\hline $\mathrm{C}$ & 0.9168 & 0.40721 & 1.06613 \\
\hline $\mathrm{N}$ & 0.92895 & 0.44237 & 1.02454 \\
\hline $\mathrm{C}$ & 0.9448 & 0.44364 & 1.02493 \\
\hline $\mathrm{C}$ & 0.95351 & 0.4544 & 1.00712 \\
\hline $\mathrm{C}$ & 0.97051 & 0.45627 & 1.00929 \\
\hline $\mathrm{C}$ & 0.97914 & 0.46587 & 0.99208 \\
\hline $\mathrm{C}$ & 0.97094 & 0.47377 & 0.97227 \\
\hline $\mathrm{C}$ & 0.95387 & 0.47206 & 0.97035 \\
\hline $\mathrm{C}$ & 0.94523 & 0.46249 & 0.98761 \\
\hline $\mathrm{C}$ & 0.98018 & 0.48361 & 0.95355 \\
\hline $\mathrm{C}$ & 0.99336 & 0.4935 & 0.96109 \\
\hline $\mathrm{C}$ & 1.00205 & 0.50276 & 0.94353 \\
\hline $\mathrm{C}$ & 0.99779 & 0.50227 & 0.91799 \\
\hline $\mathrm{C}$ & 0.98471 & 0.49231 & 0.91036 \\
\hline $\mathrm{C}$ & 0.97598 & 0.48309 & 0.92794 \\
\hline $\mathrm{N}$ & 0.39115 & -0.10708 & 0.62322 \\
\hline $\mathrm{C}$ & 0.79158 & -0.03011 & 0.87923 \\
\hline $\mathrm{C}$ & 0.78357 & -0.01517 & 0.87899 \\
\hline $\mathrm{C}$ & 0.74985 & -0.04187 & 0.88007 \\
\hline $\mathrm{C}$ & 0.73484 & -0.03365 & 0.87963 \\
\hline $\mathrm{C}$ & 0.7082 & -0.02983 & 0.87895 \\
\hline $\mathrm{C}$ & 0.69003 & 0.00022 & 0.87799 \\
\hline $\mathrm{C}$ & 0.68102 & -0.00316 & 0.89981 \\
\hline $\mathrm{C}$ & 0.66393 & -0.0031 & 0.89917 \\
\hline $\mathrm{C}$ & 0.6556 & 0.00066 & 0.87692 \\
\hline $\mathrm{C}$ & 0.66463 & 0.00389 & 0.85494 \\
\hline $\mathrm{C}$ & 0.68173 & 0.00365 & 0.85552 \\
\hline $\mathrm{N}$ & 0.63804 & 0.00055 & 0.87717 \\
\hline $\mathrm{C}$ & 0.62873 & 0.00865 & 0.8618 \\
\hline $\mathrm{C}$ & 0.61077 & 0.00749 & 0.86361 \\
\hline $\mathrm{C}$ & 0.601 & 0.01821 & 0.84954 \\
\hline $\mathrm{C}$ & 0.58396 & 0.01738 & 0.85107 \\
\hline $\mathrm{C}$ & 0.57638 & 0.00572 & 0.86659 \\
\hline
\end{tabular}




\begin{tabular}{|c|c|c|c|}
\hline $\mathrm{C}$ & 0.58618 & -0.00511 & 0.88051 \\
\hline $\mathrm{C}$ & 0.60321 & -0.00421 & 0.87911 \\
\hline $\mathrm{C}$ & 0.5583 & 0.00483 & 0.86819 \\
\hline $\mathrm{C}$ & 0.5489 & 0.01921 & 0.86964 \\
\hline $\mathrm{C}$ & 0.53186 & 0.01836 & 0.87098 \\
\hline $\mathrm{C}$ & 0.52394 & 0.00314 & 0.87068 \\
\hline $\mathrm{C}$ & 0.53328 & -0.01123 & 0.86929 \\
\hline $\mathrm{C}$ & 0.55032 & -0.01041 & 0.86817 \\
\hline $\mathrm{N}$ & 0.50635 & -0.00227 & 0.3712 \\
\hline $\mathrm{C}$ & 0.38285 & 0.1159 & 1.0709 \\
\hline $\mathrm{C}$ & 0.36809 & 0.13218 & 1.04699 \\
\hline $\mathrm{C}$ & 0.34968 & 0.14805 & 0.14604 \\
\hline $\mathrm{C}$ & 0.33761 & 0.15909 & 0.1393 \\
\hline $\mathrm{C}$ & 0.35274 & 0.14595 & 1.08033 \\
\hline $\mathrm{C}$ & 0.34377 & 0.15577 & 1.06128 \\
\hline $\mathrm{C}$ & 0.33012 & 0.14923 & 1.04885 \\
\hline $\mathrm{C}$ & 0.32144 & 0.15871 & 1.0315 \\
\hline $\mathrm{C}$ & 0.32612 & 0.17489 & 1.02657 \\
\hline $\mathrm{C}$ & 0.34001 & 0.18135 & 1.03872 \\
\hline $\mathrm{C}$ & 0.34871 & 0.17184 & 1.05599 \\
\hline $\mathrm{N}$ & 0.31684 & 0.18413 & 1.0086 \\
\hline $\mathrm{C}$ & 0.31654 & 0.19998 & 1.00677 \\
\hline $\mathrm{C}$ & 0.30689 & 0.20808 & 0.98694 \\
\hline $\mathrm{C}$ & 0.30683 & 0.22521 & 0.98539 \\
\hline $\mathrm{C}$ & 0.29807 & 0.23313 & 0.96641 \\
\hline $\mathrm{C}$ & 0.28916 & 0.22403 & 0.94862 \\
\hline $\mathrm{C}$ & 0.28913 & 0.20687 & 0.95036 \\
\hline $\mathrm{C}$ & 0.29791 & 0.19897 & 0.96931 \\
\hline $\mathrm{C}$ & 0.27998 & 0.2324 & 0.92829 \\
\hline $\mathrm{C}$ & 0.27144 & 0.24699 & 0.93315 \\
\hline $\mathrm{C}$ & 0.26281 & 0.25486 & 0.91402 \\
\hline $\mathrm{C}$ & 0.26254 & 0.24832 & 0.88963 \\
\hline $\mathrm{C}$ & 0.27105 & 0.23376 & 0.88471 \\
\hline $\mathrm{C}$ & 0.27969 & 0.22587 & 0.90384 \\
\hline $\mathrm{C}$ & 0.88092 & -0.38988 & 0.58378 \\
\hline $\mathrm{C}$ & 0.86704 & -0.37388 & 0.55819 \\
\hline $\mathrm{C}$ & 0.84792 & -0.35441 & 0.65549 \\
\hline $\mathrm{C}$ & 0.8363 & -0.34322 & 0.64756 \\
\hline $\mathrm{C}$ & 0.85208 & -0.35823 & 0.59003 \\
\hline $\mathrm{C}$ & 0.8438 & -0.3485 & 0.57004 \\
\hline $\mathrm{C}$ & 0.8305 & -0.35502 & 0.55669 \\
\hline $\mathrm{C}$ & 0.82212 & -0.34542 & 0.5391 \\
\hline
\end{tabular}




\begin{tabular}{|c|c|c|c|}
\hline $\mathrm{C}$ & 0.82674 & -0.32913 & 0.53485 \\
\hline $\mathrm{C}$ & 0.84037 & -0.32275 & 0.54773 \\
\hline $\mathrm{C}$ & 0.84877 & -0.33236 & 0.56521 \\
\hline $\mathrm{N}$ & 0.81749 & -0.31962 & 0.51712 \\
\hline $\mathrm{C}$ & 0.81698 & -0.30373 & 0.51621 \\
\hline $\mathrm{C}$ & 0.80713 & -0.29519 & 0.497 \\
\hline $\mathrm{C}$ & 0.80609 & -0.27806 & 0.49752 \\
\hline $\mathrm{C}$ & 0.79686 & -0.26969 & 0.47952 \\
\hline $\mathrm{C}$ & 0.78852 & -0.27831 & 0.46058 \\
\hline $\mathrm{C}$ & 0.78959 & -0.29549 & 0.4601 \\
\hline $\mathrm{C}$ & 0.79878 & -0.30385 & 0.47814 \\
\hline $\mathrm{C}$ & 0.77866 & -0.26945 & 0.44152 \\
\hline $\mathrm{C}$ & 0.769 & -0.25596 & 0.44849 \\
\hline $\mathrm{C}$ & 0.75957 & -0.24773 & 0.43063 \\
\hline $\mathrm{C}$ & 0.7596 & -0.25278 & 0.40541 \\
\hline $\mathrm{C}$ & 0.76932 & -0.26618 & 0.39836 \\
\hline $\mathrm{C}$ & 0.77875 & -0.27444 & 0.41623 \\
\hline $\mathrm{N}$ & 0.3568 & 0.14143 & 1.12541 \\
\hline $\mathrm{C}$ & 0.27904 & -0.4577 & 0.37967 \\
\hline $\mathrm{C}$ & 0.26431 & -0.46609 & 0.37934 \\
\hline $\mathrm{C}$ & 0.29181 & -0.4991 & 0.38275 \\
\hline $\mathrm{C}$ & 0.284 & 0.48567 & 0.38175 \\
\hline $\mathrm{C}$ & 0.28085 & 0.45893 & 0.3801 \\
\hline $\mathrm{C}$ & 1.25116 & 0.44006 & 0.37714 \\
\hline $\mathrm{C}$ & 0.25458 & 0.43074 & 0.39865 \\
\hline $\mathrm{C}$ & 0.25405 & 0.41366 & 0.39762 \\
\hline $\mathrm{C}$ & 1.24972 & 0.40565 & 0.37534 \\
\hline $\mathrm{C}$ & 1.24652 & 0.41496 & 0.35364 \\
\hline $\mathrm{C}$ & 1.24728 & 0.43205 & 0.35458 \\
\hline $\mathrm{N}$ & 1.24923 & 0.38811 & 0.37527 \\
\hline $\mathrm{C}$ & 1.24045 & 0.37914 & 0.36028 \\
\hline $\mathrm{C}$ & 1.24121 & 0.36114 & 0.36178 \\
\hline $\mathrm{C}$ & 1.22986 & 0.35165 & 0.34842 \\
\hline $\mathrm{C}$ & 1.23048 & 0.33456 & 0.34955 \\
\hline $\mathrm{C}$ & 1.24257 & 0.32668 & 0.36392 \\
\hline $\mathrm{C}$ & 0.25392 & 0.33622 & 0.37728 \\
\hline $\mathrm{C}$ & 0.25323 & 0.35327 & 0.37627 \\
\hline $\mathrm{C}$ & 1.24343 & 0.30857 & 0.36501 \\
\hline $\mathrm{C}$ & 1.2291 & 0.29917 & 0.3673 \\
\hline $\mathrm{C}$ & 1.23 & 0.28213 & 0.36887 \\
\hline $\mathrm{C}$ & 1.24521 & 0.2742 & 0.36798 \\
\hline $\mathrm{C}$ & 0.25951 & 0.28353 & 0.36546 \\
\hline
\end{tabular}




\begin{tabular}{|c|c|c|c|}
\hline $\mathrm{C}$ & 0.25864 & 0.30056 & 0.36405 \\
\hline $\mathrm{N}$ & 0.25384 & 0.25664 & 0.86982 \\
\hline $\mathrm{C}$ & 0.63645 & 0.36819 & 0.68868 \\
\hline $\mathrm{C}$ & 0.62042 & 0.38214 & 0.71409 \\
\hline $\mathrm{C}$ & 0.60513 & 0.40539 & 0.61719 \\
\hline $\mathrm{C}$ & 0.59436 & 0.41742 & 0.62512 \\
\hline $\mathrm{C}$ & 0.60672 & 0.39902 & 0.6824 \\
\hline $\mathrm{C}$ & 0.59669 & 0.40702 & 0.70223 \\
\hline $\mathrm{C}$ & 0.60311 & 0.41988 & 0.71664 \\
\hline $\mathrm{C}$ & 0.59336 & 0.42775 & 0.73456 \\
\hline $\mathrm{C}$ & 0.57702 & 0.42307 & 0.73809 \\
\hline $\mathrm{C}$ & 0.57068 & 0.40998 & 0.72389 \\
\hline $\mathrm{C}$ & 0.58045 & 0.40208 & 0.70606 \\
\hline $\mathrm{N}$ & 0.56751 & 0.43152 & 0.75675 \\
\hline $\mathrm{C}$ & 0.55163 & 0.43184 & 0.758 \\
\hline $\mathrm{C}$ & 0.5432 & 0.44051 & 0.77861 \\
\hline $\mathrm{C}$ & 0.52605 & 0.44047 & 0.77956 \\
\hline $\mathrm{C}$ & 0.51781 & 0.44825 & 0.79924 \\
\hline $\mathrm{C}$ & 0.52657 & 0.45629 & 0.81835 \\
\hline $\mathrm{C}$ & 0.54376 & 0.45646 & 0.81719 \\
\hline $\mathrm{C}$ & 0.55199 & 0.44864 & 0.79756 \\
\hline $\mathrm{C}$ & 0.51785 & 0.46445 & 0.83943 \\
\hline $\mathrm{C}$ & 0.50315 & 0.47296 & 0.83517 \\
\hline $\mathrm{C}$ & 0.49503 & 0.4807 & 0.85496 \\
\hline $\mathrm{C}$ & 0.50142 & 0.48013 & 0.87944 \\
\hline $\mathrm{C}$ & 0.51604 & 0.47159 & 0.88377 \\
\hline $\mathrm{C}$ & 0.52417 & 0.46382 & 0.86399 \\
\hline $\mathrm{C}$ & 1.1344 & -0.13308 & 0.17778 \\
\hline $\mathrm{C}$ & 1.11822 & -0.11827 & 0.20172 \\
\hline $\mathrm{C}$ & 1.10224 & -0.09993 & 1.10261 \\
\hline $\mathrm{C}$ & 1.09116 & -0.08785 & 1.10931 \\
\hline $\mathrm{C}$ & 1.10434 & -0.103 & 0.16834 \\
\hline $\mathrm{C}$ & 1.09458 & -0.09399 & 0.18741 \\
\hline $\mathrm{C}$ & 1.10144 & -0.08079 & 0.20055 \\
\hline $\mathrm{C}$ & 1.09203 & -0.07204 & 0.21788 \\
\hline $\mathrm{C}$ & 1.07563 & -0.0762 & 0.22207 \\
\hline $\mathrm{C}$ & 1.06887 & -0.08969 & 0.20927 \\
\hline $\mathrm{C}$ & 1.0783 & -0.09846 & 0.19202 \\
\hline $\mathrm{N}$ & 1.06642 & -0.0667 & 0.23981 \\
\hline $\mathrm{C}$ & 1.05056 & -0.06586 & 0.24094 \\
\hline $\mathrm{C}$ & 1.04245 & -0.05581 & 0.26025 \\
\hline $\mathrm{C}$ & 1.02535 & -0.05448 & 0.26022 \\
\hline
\end{tabular}




\begin{tabular}{|c|c|c|c|}
\hline $\mathrm{C}$ & 1.0174 & -0.04505 & 0.27837 \\
\hline $\mathrm{C}$ & 1.02642 & -0.03679 & 0.29695 \\
\hline $\mathrm{C}$ & 1.04355 & -0.03814 & 0.29695 \\
\hline $\mathrm{C}$ & 1.0515 & -0.04754 & 0.27876 \\
\hline $\mathrm{C}$ & 1.018 & -0.02672 & 0.31615 \\
\hline $\mathrm{C}$ & 1.00465 & -0.0168 & 0.30944 \\
\hline $\mathrm{C}$ & 0.99677 & -0.00725 & 0.32747 \\
\hline $\mathrm{C}$ & 1.00204 & -0.00741 & 0.35257 \\
\hline $\mathrm{C}$ & 1.01534 & -0.01733 & 0.35935 \\
\hline $\mathrm{C}$ & 1.02326 & -0.02689 & 0.34131 \\
\hline $\mathrm{N}$ & 0.6116 & 0.3973 & 0.63707 \\
\hline $\mathrm{C}$ & 1.20896 & 0.46917 & 0.38101 \\
\hline $\mathrm{C}$ & 1.21669 & 0.48425 & 0.38158 \\
\hline $\mathrm{C}$ & 0.2509 & 0.45826 & 0.37852 \\
\hline $\mathrm{C}$ & 0.26579 & 0.46668 & 0.37966 \\
\hline $\mathrm{C}$ & 0.29236 & 0.47092 & 0.38167 \\
\hline $\mathrm{C}$ & 0.31003 & -0.49856 & 0.3846 \\
\hline $\mathrm{C}$ & 0.31979 & 0.49794 & 0.36357 \\
\hline $\mathrm{C}$ & 0.33683 & 0.49915 & 0.36509 \\
\hline $\mathrm{C}$ & 0.34437 & -0.49576 & 0.38738 \\
\hline $\mathrm{C}$ & 0.33464 & -0.49244 & 0.40859 \\
\hline $\mathrm{C}$ & 0.3176 & -0.49388 & 0.40718 \\
\hline $\mathrm{N}$ & 0.3619 & -0.4947 & 0.38807 \\
\hline $\mathrm{C}$ & 0.37029 & -0.48515 & 0.40273 \\
\hline $\mathrm{C}$ & 0.38835 & -0.48542 & 0.40228 \\
\hline $\mathrm{C}$ & 0.39716 & -0.4733 & 0.41513 \\
\hline $\mathrm{C}$ & 0.41428 & -0.47352 & 0.4153 \\
\hline $\mathrm{C}$ & 0.42288 & -0.48595 & 0.40268 \\
\hline $\mathrm{C}$ & 0.414 & 0.50198 & 0.3897 \\
\hline $\mathrm{C}$ & 0.39692 & 0.50225 & 0.38946 \\
\hline $\mathrm{C}$ & 0.44104 & -0.4864 & 0.40292 \\
\hline $\mathrm{C}$ & 0.45022 & -0.47194 & 0.40024 \\
\hline $\mathrm{C}$ & 0.4673 & -0.47255 & 0.39938 \\
\hline $\mathrm{C}$ & 0.47551 & -0.48758 & 0.40142 \\
\hline $\mathrm{C}$ & 0.4664 & 0.49803 & 0.40458 \\
\hline $\mathrm{C}$ & 0.44934 & 0.49859 & 0.40527 \\
\hline $\mathrm{N}$ & 0.4931 & 0.48828 & 0.89983 \\
\hline $\mathrm{C}$ & 0.61786 & -0.38683 & 0.18938 \\
\hline $\mathrm{C}$ & 0.63222 & -0.37039 & 0.21367 \\
\hline $\mathrm{C}$ & 0.6518 & -0.35486 & 1.11511 \\
\hline $\mathrm{C}$ & 0.66351 & -0.34347 & 1.12205 \\
\hline $\mathrm{C}$ & 0.64837 & -0.35708 & 0.18078 \\
\hline
\end{tabular}




\begin{tabular}{|c|c|c|c|}
\hline $\mathrm{C}$ & 0.65725 & -0.34731 & 0.20007 \\
\hline $\mathrm{C}$ & 0.67092 & -0.35384 & 0.21246 \\
\hline $\mathrm{C}$ & 0.67946 & -0.34445 & 0.23012 \\
\hline $\mathrm{C}$ & 0.6746 & -0.32838 & 0.23547 \\
\hline $\mathrm{C}$ & 0.66064 & -0.32197 & 0.22342 \\
\hline $\mathrm{C}$ & 0.65209 & -0.33137 & 0.20583 \\
\hline $\mathrm{N}$ & 0.68387 & -0.31915 & 0.25356 \\
\hline $\mathrm{C}$ & 0.68394 & -0.3033 & 0.25568 \\
\hline $\mathrm{C}$ & 0.69381 & -0.29505 & 0.27518 \\
\hline $\mathrm{C}$ & 0.69398 & -0.27791 & 0.27622 \\
\hline $\mathrm{C}$ & 0.70318 & -0.26977 & 0.29447 \\
\hline $\mathrm{C}$ & 0.71245 & -0.27861 & 0.3121 \\
\hline $\mathrm{C}$ & 0.71224 & -0.29582 & 0.31108 \\
\hline $\mathrm{C}$ & 0.70302 & -0.30396 & 0.2928 \\
\hline $\mathrm{C}$ & 0.72231 & -0.2699 & 0.33141 \\
\hline $\mathrm{C}$ & 0.73075 & -0.25545 & 0.32527 \\
\hline $\mathrm{C}$ & 0.73986 & -0.24713 & 0.34344 \\
\hline $\mathrm{C}$ & 0.74075 & -0.25307 & 0.36815 \\
\hline $\mathrm{C}$ & 0.7324 & -0.26754 & 0.37432 \\
\hline $\mathrm{C}$ & 0.72328 & -0.27588 & 0.35615 \\
\hline $\mathrm{C}$ & 1.1157 & 0.1146 & 0.6766 \\
\hline $\mathrm{C}$ & 1.13005 & 0.12996 & 0.70248 \\
\hline $\mathrm{C}$ & 1.15075 & 0.14867 & 0.60567 \\
\hline $\mathrm{C}$ & 1.16266 & 0.15949 & 0.61379 \\
\hline $\mathrm{C}$ & 1.14629 & 0.14455 & 0.67099 \\
\hline $\mathrm{C}$ & 1.15491 & 0.15373 & 0.69116 \\
\hline $\mathrm{C}$ & 1.16809 & 0.14664 & 0.70408 \\
\hline $\mathrm{C}$ & 1.17677 & 0.15571 & 0.72196 \\
\hline $\mathrm{C}$ & 1.17256 & 0.17202 & 0.72693 \\
\hline $\mathrm{C}$ & 1.15895 & 0.17892 & 0.7146 \\
\hline $\mathrm{C}$ & 1.15026 & 0.16984 & 0.6968 \\
\hline $\mathrm{N}$ & 1.18227 & 0.18111 & 0.74457 \\
\hline $\mathrm{C}$ & 1.18329 & 0.19698 & 0.74542 \\
\hline $\mathrm{C}$ & 1.19369 & 0.20525 & 0.76414 \\
\hline $\mathrm{C}$ & 1.19527 & 0.22233 & 0.76325 \\
\hline $\mathrm{C}$ & 1.20513 & 0.23051 & 0.78058 \\
\hline $\mathrm{C}$ & 1.21363 & 0.22177 & 0.7992 \\
\hline $\mathrm{C}$ & 1.21198 & 0.20465 & 0.80017 \\
\hline $\mathrm{C}$ & 1.20212 & 0.19646 & 0.78278 \\
\hline $\mathrm{C}$ & 1.22422 & 0.23052 & 0.81742 \\
\hline $\mathrm{C}$ & 1.23406 & 0.24365 & 0.80947 \\
\hline $\mathrm{C}$ & 1.24385 & 0.25207 & 0.82661 \\
\hline
\end{tabular}




\begin{tabular}{|c|c|c|c|}
\hline $\mathrm{C}$ & 1.24402 & 0.24759 & 0.85207 \\
\hline $\mathrm{C}$ & 1.23427 & 0.23443 & 0.86004 \\
\hline $\mathrm{C}$ & 1.22448 & 0.22595 & 0.84288 \\
\hline $\mathrm{N}$ & 0.64491 & -0.36192 & 0.13562 \\
\hline $\mathrm{C}$ & 0.7202 & 0.04185 & 0.87965 \\
\hline $\mathrm{C}$ & 0.73508 & 0.03375 & 0.87982 \\
\hline $\mathrm{C}$ & 0.70824 & 0.00014 & 0.87862 \\
\hline $\mathrm{C}$ & 0.71632 & -0.01495 & 0.87897 \\
\hline $\mathrm{C}$ & 0.7199 & -0.04164 & 0.87954 \\
\hline $\mathrm{C}$ & 0.7498 & -0.06013 & 0.88063 \\
\hline $\mathrm{C}$ & 0.74628 & -0.06911 & 0.8588 \\
\hline $\mathrm{C}$ & 0.74618 & -0.08624 & 0.85937 \\
\hline $\mathrm{C}$ & 0.7499 & -0.09466 & 0.88156 \\
\hline $\mathrm{C}$ & 0.75328 & -0.08567 & 0.90354 \\
\hline $\mathrm{C}$ & 0.7532 & -0.06855 & 0.90302 \\
\hline $\mathrm{N}$ & 0.74958 & -0.11224 & 0.88127 \\
\hline $\mathrm{C}$ & 0.75756 & -0.12169 & 0.89661 \\
\hline $\mathrm{C}$ & 0.75619 & -0.13967 & 0.89483 \\
\hline $\mathrm{C}$ & 0.76681 & -0.14954 & 0.90893 \\
\hline $\mathrm{C}$ & 0.7658 & -0.16659 & 0.90749 \\
\hline $\mathrm{C}$ & 0.75405 & -0.1741 & 0.89203 \\
\hline $\mathrm{C}$ & 0.74332 & -0.16419 & 0.87808 \\
\hline $\mathrm{C}$ & 0.74442 & -0.14714 & 0.87938 \\
\hline $\mathrm{C}$ & 0.75302 & -0.1922 & 0.89047 \\
\hline $\mathrm{C}$ & 0.76732 & -0.20171 & 0.88899 \\
\hline $\mathrm{C}$ & 0.76636 & -0.21875 & 0.8876 \\
\hline $\mathrm{C}$ & 0.7511 & -0.2266 & 0.88789 \\
\hline $\mathrm{C}$ & 0.7368 & -0.21716 & 0.88936 \\
\hline $\mathrm{C}$ & 0.73774 & -0.2001 & 0.89051 \\
\hline $\mathrm{N}$ & 0.74986 & -0.24421 & 0.387 \\
\hline $\mathrm{N}$ & 0.3735 & -0.12475 & 0.58698 \\
\hline $\mathrm{N}$ & 0.62808 & -0.37995 & 1.09882 \\
\hline $\mathrm{N}$ & 0.62757 & 0.37815 & 0.67352 \\
\hline $\mathrm{N}$ & 0.37445 & 0.12376 & 0.16169 \\
\hline $\mathrm{N}$ & 0.76712 & -0.01729 & 0.87941 \\
\hline $\mathrm{N}$ & 0.26683 & -0.48247 & 0.38046 \\
\hline $\mathrm{N}$ & 0.73275 & -0.01718 & 0.87931 \\
\hline $\mathrm{N}$ & 0.26761 & 0.48317 & 0.38065 \\
\hline $\mathrm{H}$ & 0.38544 & -0.11564 & 0.52935 \\
\hline $\mathrm{H}$ & 0.4167 & -0.07947 & 0.64607 \\
\hline $\mathrm{H}$ & 0.38472 & -0.07739 & 0.55029 \\
\hline $\mathrm{H}$ & 0.40076 & -0.06249 & 0.51842 \\
\hline
\end{tabular}




\begin{tabular}{|c|c|c|c|}
\hline $\mathrm{H}$ & 0.44035 & -0.0969 & 0.52806 \\
\hline $\mathrm{H}$ & 0.42445 & -0.11145 & 0.55985 \\
\hline $\mathrm{H}$ & 0.45438 & -0.07549 & 0.51426 \\
\hline $\mathrm{H}$ & 0.47934 & -0.06561 & 0.49423 \\
\hline $\mathrm{H}$ & 0.49363 & -0.04948 & 0.46247 \\
\hline $\mathrm{H}$ & 0.44722 & -0.03275 & 0.43333 \\
\hline $\mathrm{H}$ & 0.43297 & -0.04922 & 0.46475 \\
\hline $\mathrm{H}$ & 0.49948 & -0.02179 & 0.45167 \\
\hline $\mathrm{H}$ & 0.51422 & -0.0059 & 0.42007 \\
\hline $\mathrm{H}$ & 0.47825 & -0.01919 & 0.36448 \\
\hline $\mathrm{H}$ & 0.46347 & -0.03523 & 0.39596 \\
\hline $\mathrm{H}$ & 0.88359 & 0.39201 & 1.04241 \\
\hline $\mathrm{H}$ & 0.91339 & 0.4243 & 0.16268 \\
\hline $\mathrm{H}$ & 0.88182 & 0.42987 & 1.06741 \\
\hline $\mathrm{H}$ & 0.89812 & 0.44696 & 1.03849 \\
\hline $\mathrm{H}$ & 0.93838 & 0.41315 & 1.04534 \\
\hline $\mathrm{H}$ & 0.92212 & 0.39615 & 1.07377 \\
\hline $\mathrm{H}$ & 0.95194 & 0.43692 & 1.03863 \\
\hline $\mathrm{H}$ & 0.97715 & 0.45017 & 1.0242 \\
\hline $\mathrm{H}$ & 0.9923 & 0.4668 & 0.99397 \\
\hline $\mathrm{H}$ & 0.94715 & 0.4782 & 0.95556 \\
\hline $\mathrm{H}$ & 0.93211 & 0.46131 & 0.98553 \\
\hline $\mathrm{H}$ & 0.99685 & 0.49423 & 0.98063 \\
\hline $\mathrm{H}$ & 1.01205 & 0.51041 & 0.94986 \\
\hline $\mathrm{H}$ & 0.9814 & 0.4915 & 0.8907 \\
\hline $\mathrm{H}$ & 0.96612 & 0.47532 & 0.92143 \\
\hline $\mathrm{H}$ & 0.80462 & -0.03242 & 0.87917 \\
\hline $\mathrm{H}$ & 0.69515 & -0.03206 & 0.87857 \\
\hline $\mathrm{H}$ & 0.68725 & -0.00589 & 0.91726 \\
\hline $\mathrm{H}$ & 0.65712 & -0.00582 & 0.91612 \\
\hline $\mathrm{H}$ & 0.65857 & 0.00629 & 0.83726 \\
\hline $\mathrm{H}$ & 0.68852 & 0.0061 & 0.83847 \\
\hline $\mathrm{H}$ & 0.63399 & 0.01687 & 0.84804 \\
\hline $\mathrm{H}$ & 0.60656 & 0.02729 & 0.83741 \\
\hline $\mathrm{H}$ & 0.57671 & 0.02573 & 0.83986 \\
\hline $\mathrm{H}$ & 0.58066 & -0.01415 & 0.89274 \\
\hline $\mathrm{H}$ & 0.61045 & -0.01269 & 0.8901 \\
\hline $\mathrm{H}$ & 0.55474 & 0.0311 & 0.86993 \\
\hline $\mathrm{H}$ & 0.5248 & 0.02955 & 0.87189 \\
\hline $\mathrm{H}$ & 0.52732 & -0.02307 & 0.8691 \\
\hline $\mathrm{H}$ & 0.55724 & -0.02168 & 0.86686 \\
\hline $\mathrm{H}$ & 0.36304 & 0.13837 & 1.03077 \\
\hline
\end{tabular}




\begin{tabular}{|c|c|c|c|}
\hline $\mathrm{H}$ & 0.3297 & 0.16615 & 0.15145 \\
\hline $\mathrm{H}$ & 0.32617 & 0.13684 & 1.05273 \\
\hline $\mathrm{H}$ & 0.31092 & 0.15351 & 1.02206 \\
\hline $\mathrm{H}$ & 0.34436 & 0.19357 & 1.03476 \\
\hline $\mathrm{H}$ & 0.35929 & 0.177 & 1.0653 \\
\hline $\mathrm{H}$ & 0.32319 & 0.20755 & 1.01997 \\
\hline $\mathrm{H}$ & 0.31369 & 0.23249 & 0.99875 \\
\hline $\mathrm{H}$ & 0.29849 & 0.24635 & 0.9654 \\
\hline $\mathrm{H}$ & 0.28218 & 0.19953 & 0.9372 \\
\hline $\mathrm{H}$ & 0.29767 & 0.18573 & 0.97011 \\
\hline $\mathrm{H}$ & 0.27125 & 0.25223 & 0.95183 \\
\hline $\mathrm{H}$ & 0.2562 & 0.266 & 0.91826 \\
\hline $\mathrm{H}$ & 0.27118 & 0.22863 & 0.86595 \\
\hline $\mathrm{H}$ & 0.28637 & 0.2148 & 0.89939 \\
\hline $\mathrm{H}$ & 0.86234 & -0.36817 & 0.54133 \\
\hline $\mathrm{H}$ & 0.82835 & -0.33566 & 0.65894 \\
\hline $\mathrm{H}$ & 0.82649 & -0.36745 & 0.56017 \\
\hline $\mathrm{H}$ & 0.81179 & -0.35058 & 0.52913 \\
\hline $\mathrm{H}$ & 0.84469 & -0.31044 & 0.54431 \\
\hline $\mathrm{H}$ & 0.85907 & -0.32719 & 0.57524 \\
\hline $\mathrm{H}$ & 0.82339 & -0.29641 & 0.53 \\
\hline $\mathrm{H}$ & 0.81244 & -0.27112 & 0.51185 \\
\hline $\mathrm{H}$ & 0.79643 & -0.25646 & 0.48025 \\
\hline $\mathrm{H}$ & 0.78311 & -0.30249 & 0.44597 \\
\hline $\mathrm{H}$ & 0.79932 & -0.31708 & 0.4773 \\
\hline $\mathrm{H}$ & 0.76851 & -0.25193 & 0.46785 \\
\hline $\mathrm{H}$ & 0.75208 & -0.2375 & 0.43653 \\
\hline $\mathrm{H}$ & 0.76973 & -0.27012 & 0.37895 \\
\hline $\mathrm{H}$ & 0.78628 & -0.2846 & 0.41019 \\
\hline $\mathrm{H}$ & 0.28105 & -0.44462 & 0.37913 \\
\hline $\mathrm{H}$ & 0.28341 & 0.44594 & 0.37952 \\
\hline $\mathrm{H}$ & 0.25754 & 0.43673 & 0.41621 \\
\hline $\mathrm{H}$ & 0.25673 & 0.40662 & 0.41436 \\
\hline $\mathrm{H}$ & 1.24366 & 0.40912 & 0.33595 \\
\hline $\mathrm{H}$ & 1.24468 & 0.43908 & 0.3378 \\
\hline $\mathrm{H}$ & 1.23206 & 0.38475 & 0.34709 \\
\hline $\mathrm{H}$ & 1.22048 & 0.35745 & 0.33713 \\
\hline $\mathrm{H}$ & 1.22167 & 0.32751 & 0.33888 \\
\hline $\mathrm{H}$ & 0.26325 & 0.33049 & 0.38874 \\
\hline $\mathrm{H}$ & 0.26217 & 0.36027 & 0.38675 \\
\hline $\mathrm{H}$ & 1.21723 & 0.30501 & 0.3683 \\
\hline $\mathrm{H}$ & 1.21885 & 0.27508 & 0.37071 \\
\hline
\end{tabular}




\begin{tabular}{|c|c|c|c|}
\hline $\mathrm{H}$ & 0.27135 & 0.27759 & 0.36486 \\
\hline $\mathrm{H}$ & 0.26987 & 0.30747 & 0.36204 \\
\hline $\mathrm{H}$ & 0.61434 & 0.38663 & 0.73079 \\
\hline $\mathrm{H}$ & 0.58754 & 0.42606 & 0.61379 \\
\hline $\mathrm{H}$ & 0.61561 & 0.42387 & 0.71388 \\
\hline $\mathrm{H}$ & 0.59847 & 0.43766 & 0.74555 \\
\hline $\mathrm{H}$ & 0.55832 & 0.40562 & 0.72665 \\
\hline $\mathrm{H}$ & 0.57535 & 0.39213 & 0.69515 \\
\hline $\mathrm{H}$ & 0.54425 & 0.42592 & 0.74378 \\
\hline $\mathrm{H}$ & 0.519 & 0.4343 & 0.76517 \\
\hline $\mathrm{H}$ & 0.50458 & 0.44772 & 0.7997 \\
\hline $\mathrm{H}$ & 0.55088 & 0.46277 & 0.83135 \\
\hline $\mathrm{H}$ & 0.56523 & 0.44895 & 0.79725 \\
\hline $\mathrm{H}$ & 0.49805 & 0.47388 & 0.81645 \\
\hline $\mathrm{H}$ & 0.48386 & 0.48738 & 0.85114 \\
\hline $\mathrm{H}$ & 0.52109 & 0.47085 & 0.90254 \\
\hline $\mathrm{H}$ & 0.5353 & 0.45714 & 0.86801 \\
\hline $\mathrm{H}$ & 1.11211 & -0.11315 & 0.21795 \\
\hline $\mathrm{H}$ & 1.08407 & -0.07995 & 1.09712 \\
\hline $\mathrm{H}$ & 1.11402 & -0.07724 & 0.19724 \\
\hline $\mathrm{H}$ & 1.09746 & -0.06184 & 0.22785 \\
\hline $\mathrm{H}$ & 1.05645 & -0.09365 & 0.21268 \\
\hline $\mathrm{H}$ & 1.0729 & -0.10873 & 0.18217 \\
\hline $\mathrm{H}$ & 1.04301 & -0.07216 & 0.22733 \\
\hline $\mathrm{H}$ & 1.01812 & -0.06079 & 0.24617 \\
\hline $\mathrm{H}$ & 1.00417 & -0.04438 & 0.27807 \\
\hline $\mathrm{H}$ & 1.05084 & -0.03174 & 0.3108 \\
\hline $\mathrm{H}$ & 1.06472 & -0.04832 & 0.27921 \\
\hline $\mathrm{H}$ & 1.00045 & -0.01625 & 0.29017 \\
\hline $\mathrm{H}$ & 0.9866 & 0.0004 & 0.32181 \\
\hline $\mathrm{H}$ & 1.01943 & -0.01782 & 0.37868 \\
\hline $\mathrm{H}$ & 1.03334 & -0.03461 & 0.34711 \\
\hline $\mathrm{H}$ & 1.19598 & 0.46658 & 0.38136 \\
\hline $\mathrm{H}$ & 0.30543 & 0.46889 & 0.38257 \\
\hline $\mathrm{H}$ & 0.31418 & 0.49437 & 0.34598 \\
\hline $\mathrm{H}$ & 0.3442 & 0.49638 & 0.34872 \\
\hline $\mathrm{H}$ & 0.34013 & -0.48898 & 0.42631 \\
\hline $\mathrm{H}$ & 0.31028 & -0.49122 & 0.42362 \\
\hline $\mathrm{H}$ & 0.36416 & -0.47651 & 0.41497 \\
\hline $\mathrm{H}$ & 0.39076 & -0.46368 & 0.42502 \\
\hline $\mathrm{H}$ & 0.42077 & -0.46413 & 0.42563 \\
\hline $\mathrm{H}$ & 0.42027 & 0.49238 & 0.3795 \\
\hline
\end{tabular}




\begin{tabular}{|c|c|c|c|}
\hline $\mathrm{H}$ & 0.39045 & 0.49273 & 0.37938 \\
\hline $\mathrm{H}$ & 0.44418 & -0.46022 & 0.39824 \\
\hline $\mathrm{H}$ & 0.47417 & -0.46132 & 0.39707 \\
\hline $\mathrm{H}$ & 0.47253 & 0.48632 & 0.40608 \\
\hline $\mathrm{H}$ & 0.44263 & 0.48728 & 0.40769 \\
\hline $\mathrm{H}$ & 0.63695 & -0.36406 & 0.23001 \\
\hline $\mathrm{H}$ & 0.67134 & -0.33617 & 1.11006 \\
\hline $\mathrm{H}$ & 0.67502 & -0.36615 & 0.2083 \\
\hline $\mathrm{H}$ & 0.69003 & -0.34963 & 0.23944 \\
\hline $\mathrm{H}$ & 0.65613 & -0.30984 & 0.22768 \\
\hline $\mathrm{H}$ & 0.64146 & -0.32624 & 0.19663 \\
\hline $\mathrm{H}$ & 0.67708 & -0.29576 & 0.2427 \\
\hline $\mathrm{H}$ & 0.68694 & -0.27077 & 0.26289 \\
\hline $\mathrm{H}$ & 0.70286 & -0.25652 & 0.29496 \\
\hline $\mathrm{H}$ & 0.71939 & -0.303 & 0.32418 \\
\hline $\mathrm{H}$ & 0.70315 & -0.31721 & 0.29244 \\
\hline $\mathrm{H}$ & 0.73046 & -0.25063 & 0.30633 \\
\hline $\mathrm{H}$ & 0.74632 & -0.23608 & 0.33819 \\
\hline $\mathrm{H}$ & 0.73273 & -0.27223 & 0.39333 \\
\hline $\mathrm{H}$ & 0.71672 & -0.28683 & 0.36159 \\
\hline $\mathrm{H}$ & 1.13482 & 0.13557 & 0.7194 \\
\hline $\mathrm{H}$ & 1.17082 & 0.16693 & 0.60256 \\
\hline $\mathrm{H}$ & 1.17178 & 0.13419 & 0.70002 \\
\hline $\mathrm{H}$ & 1.18706 & 0.15016 & 0.73154 \\
\hline $\mathrm{H}$ & 1.15491 & 0.19124 & 0.71865 \\
\hline $\mathrm{H}$ & 1.14001 & 0.17541 & 0.68719 \\
\hline $\mathrm{H}$ & 1.17699 & 0.20446 & 0.73172 \\
\hline $\mathrm{H}$ & 1.18887 & 0.22936 & 0.74907 \\
\hline $\mathrm{H}$ & 1.20596 & 0.24372 & 0.77955 \\
\hline $\mathrm{H}$ & 1.21851 & 0.19757 & 0.81413 \\
\hline $\mathrm{H}$ & 1.20114 & 0.18326 & 0.78389 \\
\hline $\mathrm{H}$ & 1.23432 & 0.24731 & 0.78993 \\
\hline $\mathrm{H}$ & 1.25138 & 0.26209 & 0.82 \\
\hline $\mathrm{H}$ & 1.23403 & 0.2309 & 0.87964 \\
\hline $\mathrm{H}$ & 1.21686 & 0.21605 & 0.84963 \\
\hline $\mathrm{H}$ & 0.71796 & 0.0549 & 0.87975 \\
\hline $\mathrm{H}$ & 0.71755 & -0.05468 & 0.87982 \\
\hline $\mathrm{H}$ & 0.74357 & -0.06284 & 0.84138 \\
\hline $\mathrm{H}$ & 0.74336 & -0.09299 & 0.8424 \\
\hline $\mathrm{H}$ & 0.75567 & -0.09178 & 0.9212 \\
\hline $\mathrm{H}$ & 0.75575 & -0.06182 & 0.9201 \\
\hline $\mathrm{H}$ & 0.76584 & -0.11653 & 0.91038 \\
\hline
\end{tabular}




\begin{tabular}{|r|r|r|r|}
\hline $\mathrm{H}$ & 0.77594 & -0.14405 & 0.92103 \\
\hline $\mathrm{H}$ & 0.77408 & -0.1739 & 0.91871 \\
\hline $\mathrm{H}$ & 0.73423 & -0.16964 & 0.86588 \\
\hline $\mathrm{H}$ & 0.73602 & -0.13983 & 0.86835 \\
\hline $\mathrm{H}$ & 0.77925 & -0.19595 & 0.88868 \\
\hline $\mathrm{H}$ & 0.77751 & -0.22588 & 0.8866 \\
\hline $\mathrm{H}$ & 0.72493 & -0.22304 & 0.88952 \\
\hline $\mathrm{H}$ & 0.72652 & -0.1931 & 0.89182 \\
\hline $\mathrm{Cu}$ & 0.37206 & 0.37943 & 0.86398 \\
\hline $\mathrm{Cu}$ & 0.37397 & 0.87575 & 0.62433 \\
\hline $\mathrm{Cu}$ & 0.25 & 0.5 & 0.88055 \\
\hline $\mathrm{Cu}$ & 0.25 & 1 & 0.62063 \\
\hline
\end{tabular}

Table S16. Fractional atomic coordinates for the unit cell of COF-2 with the interpenetrated tbo topology.

\begin{tabular}{|c|c|c|c|}
\hline \multicolumn{4}{|l|}{ Space group: $F \mathrm{~d}-3 \mathrm{~m}$} \\
\hline \multicolumn{4}{|c|}{$\mathrm{a}=\mathrm{b}=\mathrm{c}=96.0716 \AA, \alpha=\beta=\gamma=90^{\circ}$} \\
\hline Atom & $\mathrm{x}$ & $\mathrm{y}$ & $\mathrm{Z}$ \\
\hline $\mathrm{C}$ & 0.29294 & 1.02017 & 0.53801 \\
\hline $\mathrm{C}$ & 0.30336 & 1.02735 & 0.54517 \\
\hline $\mathrm{C}$ & 0.33404 & 1.07561 & 0.55778 \\
\hline $\mathrm{C}$ & 0.34526 & 1.08216 & 0.56432 \\
\hline $\mathrm{C}$ & 0.21034 & 0.97986 & 0.46202 \\
\hline $\mathrm{C}$ & 0.19978 & 0.97279 & 0.45496 \\
\hline $\mathrm{C}$ & 0.15393 & 0.93633 & 0.45416 \\
\hline $\mathrm{C}$ & 0.14297 & 0.92957 & 0.4474 \\
\hline $\mathrm{H}$ & 0.28899 & 1.01048 & 0.54218 \\
\hline $\mathrm{H}$ & 0.30741 & 1.02316 & 0.55481 \\
\hline $\mathrm{H}$ & 0.32979 & 1.08002 & 0.54832 \\
\hline $\mathrm{H}$ & 0.34958 & 1.09155 & 0.55985 \\
\hline $\mathrm{H}$ & 0.21433 & 0.98952 & 0.45782 \\
\hline $\mathrm{H}$ & 0.19569 & 0.97701 & 0.44536 \\
\hline $\mathrm{H}$ & 0.15805 & 0.93203 & 0.46373 \\
\hline $\mathrm{H}$ & 0.13872 & 0.92011 & 0.45181 \\
\hline $\mathrm{N}$ & 0.25 & 1.02234 & 0.25 \\
\hline $\mathrm{C}$ & 0.24048 & 1.0207 & 0.5207 \\
\hline
\end{tabular}




\begin{tabular}{|c|c|c|c|}
\hline $\mathrm{C}$ & 0.25874 & 1.03046 & 0.53046 \\
\hline $\mathrm{C}$ & 0.2877 & 1.02547 & 0.52547 \\
\hline $\mathrm{C}$ & 0.30857 & 1.03987 & 0.53987 \\
\hline $\mathrm{N}$ & 0.31945 & 1.04702 & 0.54702 \\
\hline $\mathrm{C}$ & 0.31689 & 1.05637 & 0.55637 \\
\hline $\mathrm{C}$ & 0.32843 & 1.06338 & 0.56338 \\
\hline $\mathrm{C}$ & 0.35093 & 1.07651 & 0.57651 \\
\hline $\mathrm{C}$ & 0.36295 & 1.08317 & 0.58317 \\
\hline $\mathrm{C}$ & 0.38596 & 1.09585 & 0.59585 \\
\hline $\mathrm{C}$ & 0.27678 & 1.01776 & 0.51776 \\
\hline $\mathrm{C}$ & 0.26285 & 1.0207 & 0.5207 \\
\hline $\mathrm{C}$ & 0.24459 & 1.03046 & 0.53046 \\
\hline $\mathrm{N}$ & 0.25166 & 1.01463 & 0.51463 \\
\hline $\mathrm{C}$ & 0.21562 & 0.97453 & 0.47453 \\
\hline $\mathrm{C}$ & 0.19442 & 0.96037 & 0.46037 \\
\hline $\mathrm{N}$ & 0.18341 & 0.95332 & 0.45332 \\
\hline $\mathrm{C}$ & 0.17049 & 0.95604 & 0.45604 \\
\hline $\mathrm{C}$ & 0.15941 & 0.94866 & 0.44866 \\
\hline $\mathrm{C}$ & 0.13742 & 0.93511 & 0.43511 \\
\hline $\mathrm{C}$ & 0.12553 & 0.92833 & 0.42833 \\
\hline $\mathrm{C}$ & 0.10271 & 0.91548 & 0.41548 \\
\hline $\mathrm{C}$ & 0.22655 & 0.98224 & 0.48224 \\
\hline $\mathrm{H}$ & 0.26527 & 1.03695 & 0.53695 \\
\hline $\mathrm{H}$ & 0.30629 & 1.05909 & 0.55909 \\
\hline $\mathrm{H}$ & 0.23806 & 1.03695 & 0.53695 \\
\hline $\mathrm{H}$ & 0.16772 & 0.96377 & 0.46377 \\
\hline $\mathrm{N}$ & 0.39768 & 1.10232 & 0.60232 \\
\hline $\mathrm{N}$ & 0.0911 & 0.9089 & 0.4089 \\
\hline $\mathrm{C}$ & 0.63903 & 1.09339 & 0.40661 \\
\hline $\mathrm{C}$ & 0.62755 & 1.0997 & 0.4003 \\
\hline $\mathrm{C}$ & 0.62353 & 1.07932 & 0.42068 \\
\hline $\mathrm{C}$ & 0.61207 & 1.08564 & 0.41436 \\
\hline $\mathrm{C}$ & 0.04473 & 1.245 & 0.255 \\
\hline $\mathrm{H}$ & 0.64943 & 1.09645 & 0.40355 \\
\hline $\mathrm{H}$ & 0.62914 & 1.10759 & 0.39241 \\
\hline $\mathrm{H}$ & 0.62188 & 1.07144 & 0.42856 \\
\hline $\mathrm{H}$ & 0.60167 & 1.08259 & 0.41741 \\
\hline $\mathrm{H}$ & 0.05391 & 1.24038 & 0.25962 \\
\hline $\mathrm{C}$ & 0.66173 & 0.66173 & 0.63372 \\
\hline $\mathrm{C}$ & 0.66813 & 0.66813 & 0.62233 \\
\hline $\mathrm{C}$ & 0.68208 & 0.68208 & 0.63806 \\
\hline $\mathrm{C}$ & 0.67567 & 0.67567 & 0.64942 \\
\hline
\end{tabular}




\begin{tabular}{|r|r|r|r|}
\hline $\mathrm{H}$ & 0.65386 & 0.65386 & 0.632 \\
\hline $\mathrm{H}$ & 0.66516 & 0.66516 & 0.61188 \\
\hline $\mathrm{H}$ & 0.68994 & 0.68994 & 0.63983 \\
\hline $\mathrm{H}$ & 0.67864 & 0.67864 & 0.65986 \\
\hline $\mathrm{C}$ & 0.24209 & 1.03093 & 0.25791 \\
\hline $\mathrm{C}$ & 0.49209 & 1.00791 & 0.7776 \\
\hline $\mathrm{C}$ & 0.495 & 1.005 & 0.7914 \\
\hline $\mathrm{H}$ & 0.49039 & 1.00961 & 0.80058 \\
\hline $\mathrm{N}$ & 0.5 & 1 & 0.76901 \\
\hline $\mathrm{Cu}$ & 0.24834 & 0.5 & 0.5 \\
\hline
\end{tabular}

\section{Figure S24. The stability test of COFs.}

The samples were immersed in different solvents at room temperature (except for boiling water) for $48 \mathrm{~h}$. After that, the powders were filtrated, washed with THF and diethyl ether, and dried in $60{ }^{\circ} \mathrm{C}$ for $6 \mathrm{~h}$.
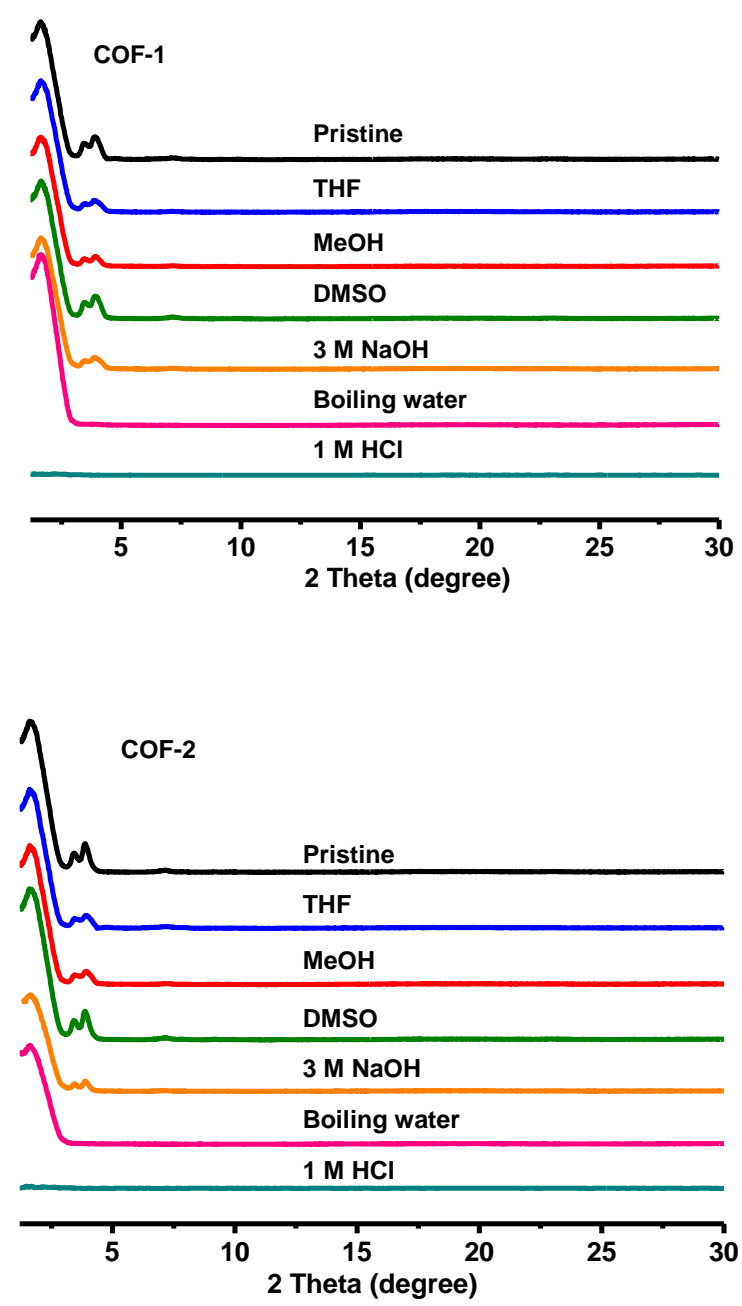


\section{Figures S25-S29 \& Table S17. Dye adsorption measurements}

\subsection{Dye uptake measurements by UV-vis}

The activated COFs ( $5 \mathrm{mg}$ ) were soaked in a THF solution of different dyes for $24 \mathrm{~h}$. To remove the dyes adsorbed on the surface of COFs, the resulted solids were washed with THF thoroughly until the washings became colorless. The solids were sonicated with solvent several times, and the solution that contained dye-A was diluted to 250 $\mathrm{mL}$, while the solution containing dye-B $(\mathbf{C})$ was diluted to $100 \mathrm{~mL}$. The absorbance of the sample solutions were determined by the UV-vis Spectrometer. The concentrations of dye can be calculated by comparing the UV-vis absorbance with a standard curve. Data for known concentrations of dyes were used to make the standard curve, plotting concentration on the $\mathrm{X}$ axis, and the assay measurement of absorbance on the $\mathrm{Y}$ axis. According to the Beer-Lambert law, the standard curve can be calculated by linear fitting of the data.

\subsection{Confocal fluorescence microscopy (CFM) experiments}

The as-obtained COFs were collected by filtration and washed with DMF and acetone. Then further purification of the resulted COFs was carried out by Soxhlet extraction in THF for $24 \mathrm{~h}$ and dried at $60{ }^{\circ} \mathrm{C}$ under vacuum overnight to afford the activated COFs. The activated COFs $(5 \mathrm{mg})$ were soaked in a solution of different dyes (A-C) for $24 \mathrm{~h}$. To remove the dye adsorbed on the surface of COFs, the obtained samples were washed with THF or EtOH thoroughly until the washings became colorless. The Confocal fluorescence microscopy images of the inclusion-dye samples were obtained by the confocal fluorescence microscopy, which reflected the local concentration of dyes.

\subsection{Substrate uptake measurements by UV-vis}

The activated COFs $(5 \mathrm{mg})$ were soaked in a EtOH solution of biphenylboric acid (4f) for $24 \mathrm{~h}$. To remove the dyes adsorbed on the surface of COFs, the resulted solids were washed with EtOH for 6 times. The solids were sonicated with solvent several times, and the solution that contained $\mathbf{4 f}$ was diluted to $100 \mathrm{~mL}$. The absorbance of the sample solutions were determined by the UV-vis Spectrometer. The concentrations of dye can be calculated by comparing the UV-vis absorbance with a standard curve. 
Figure S25. Creation of a standard curve
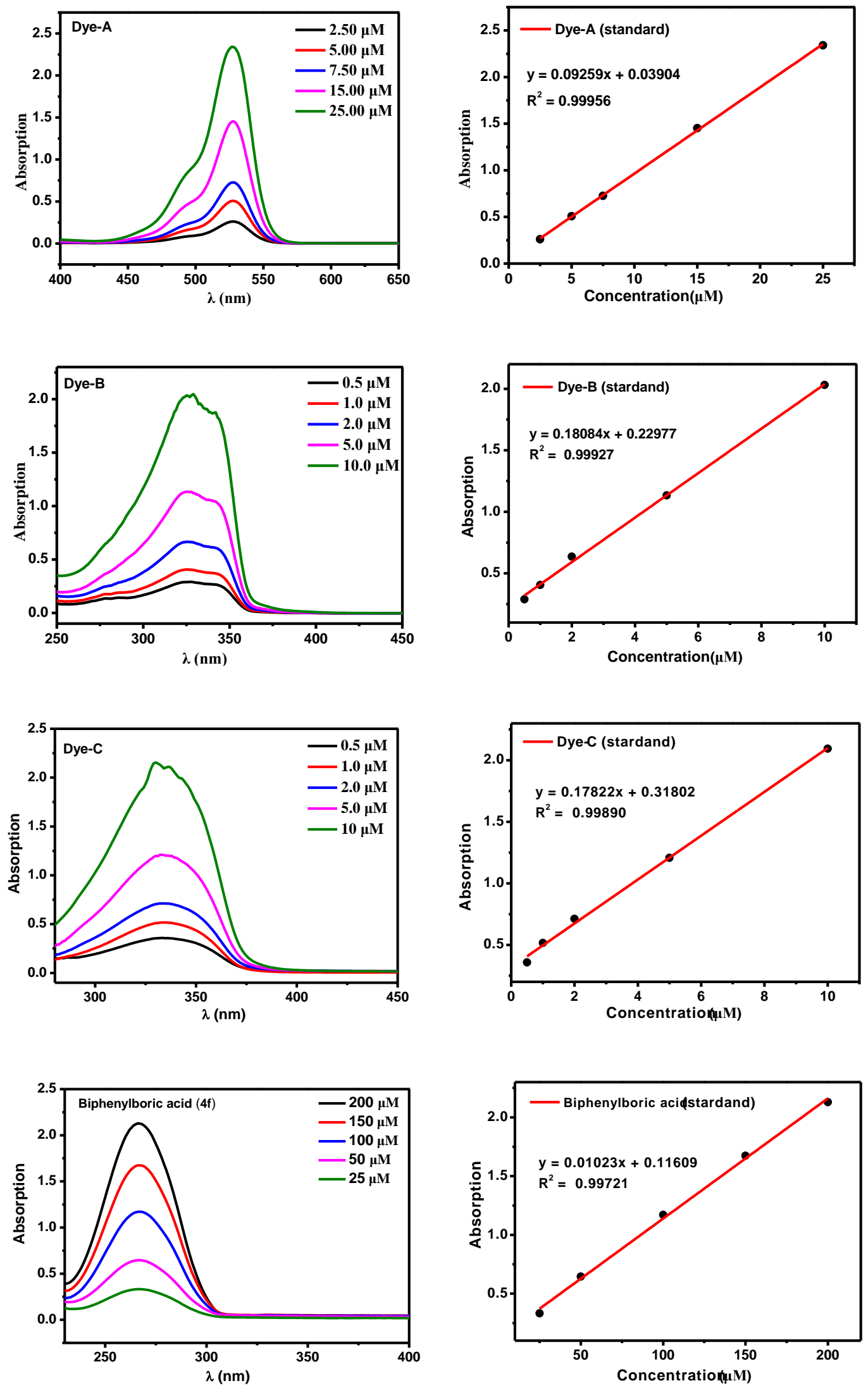
Figure S26. Adsorption curve of different dyes and $\mathbf{4 f}$
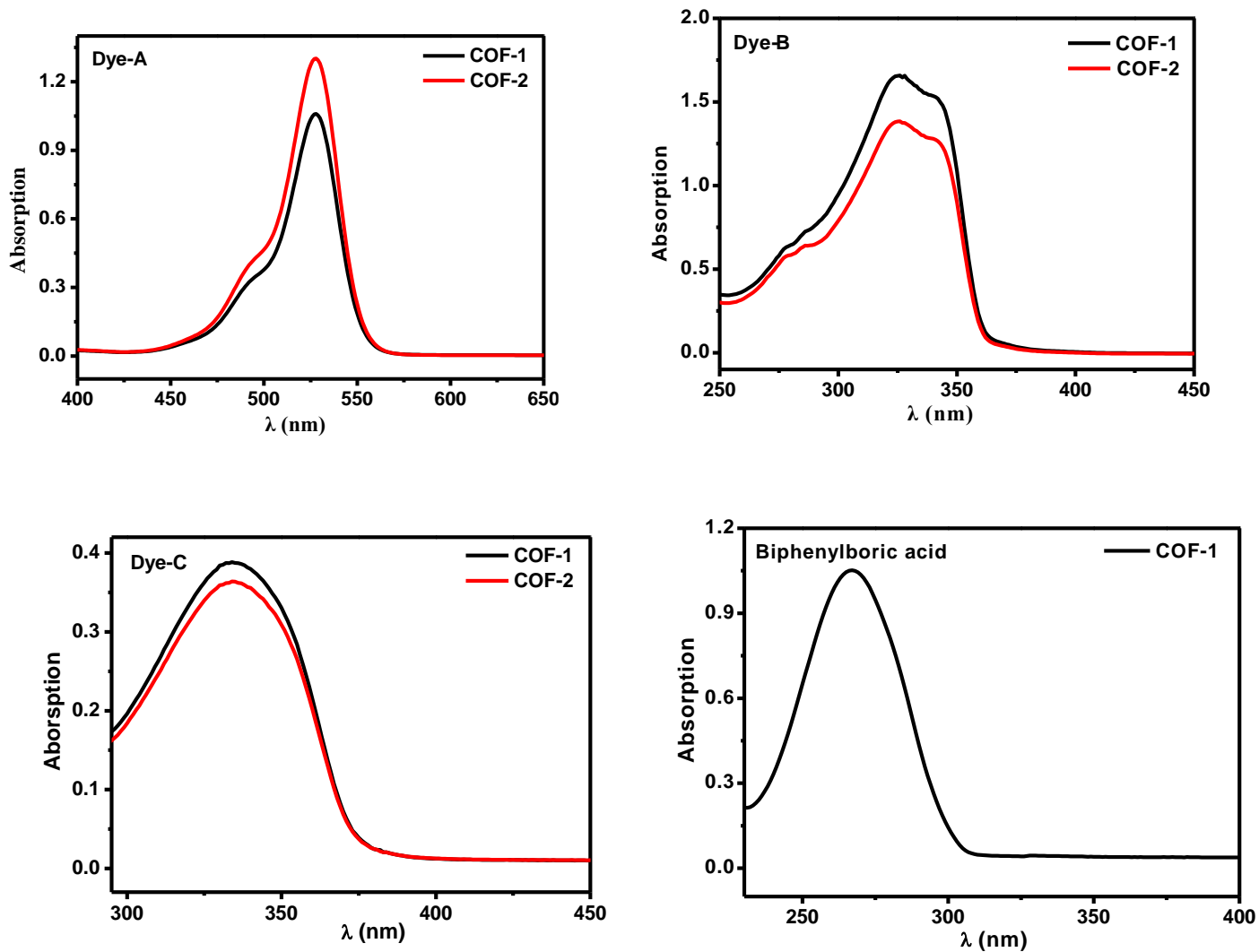

Table S17. Uptake of different dyes and $\mathbf{4} \mathbf{f}$ incubated in COFs by UV-Vis

\begin{tabular}{|c|c|c|c|c|}
\hline & $\begin{array}{l}\text { Rhodamine } \\
6 \mathrm{G} \text { (A) } \\
\text { assigned to } \\
\text { Cationic } \\
\text { organic dye }\end{array}$ & $\begin{array}{c}\left(\left(\left(\left(5^{\prime}-(4-((4-\right.\right.\right.\right. \\
\text { ((trimethylsilyl)ethynyl)ph } \\
\text { enyl)ethynyl)phenyl)- } \\
{\left[1,1^{\prime}: 3 \text { ',1"-terphenyl]- }\right.} \\
4,4^{\prime \prime} \text {-diyl)bis(ethyne-2,1- } \\
\text { diyl))bis(4,1- } \\
\text { phenylene) )bis(ethyne- } \\
\text { 2,1-diyl)) bis } \\
\text { (trimethylsilane) (B) } \\
\text { assigned to neutral } \\
\text { organic dye }\end{array}$ & $\begin{array}{l}\text { (((5'-(4-((4'-(trimethylsilyl)- } \\
{\left[1,1^{\prime}-\text { biphenyl]-4- }\right.} \\
\text { yl)ethynyl)phenyl)-[1,1':3',1"- } \\
\text { terphenyl]-4,4"-diyl)bis(ethyne- } \\
\text { 2,1-diyl))bis([1,1'-biphenyl]- } \\
\text { 4',4-diyl))bis(trimethylsilane) } \\
\text { (C) } \\
\text { assigned to neutral organic dye }\end{array}$ & $\begin{array}{c}\text { Biphen- } \\
\text { ylboric acid } \\
\text { (4f) }\end{array}$ \\
\hline COF-1 & 26.4 wt\% & 14.1 wt\% & 0.0083 wt\% & 36.1 wt\% \\
\hline COF-2 & 32.6 wt\% & 11.4 wt\% & 0.0054 wt\% & - \\
\hline
\end{tabular}

Figure S27. PXRD patterns of COFs after absorption of dye 

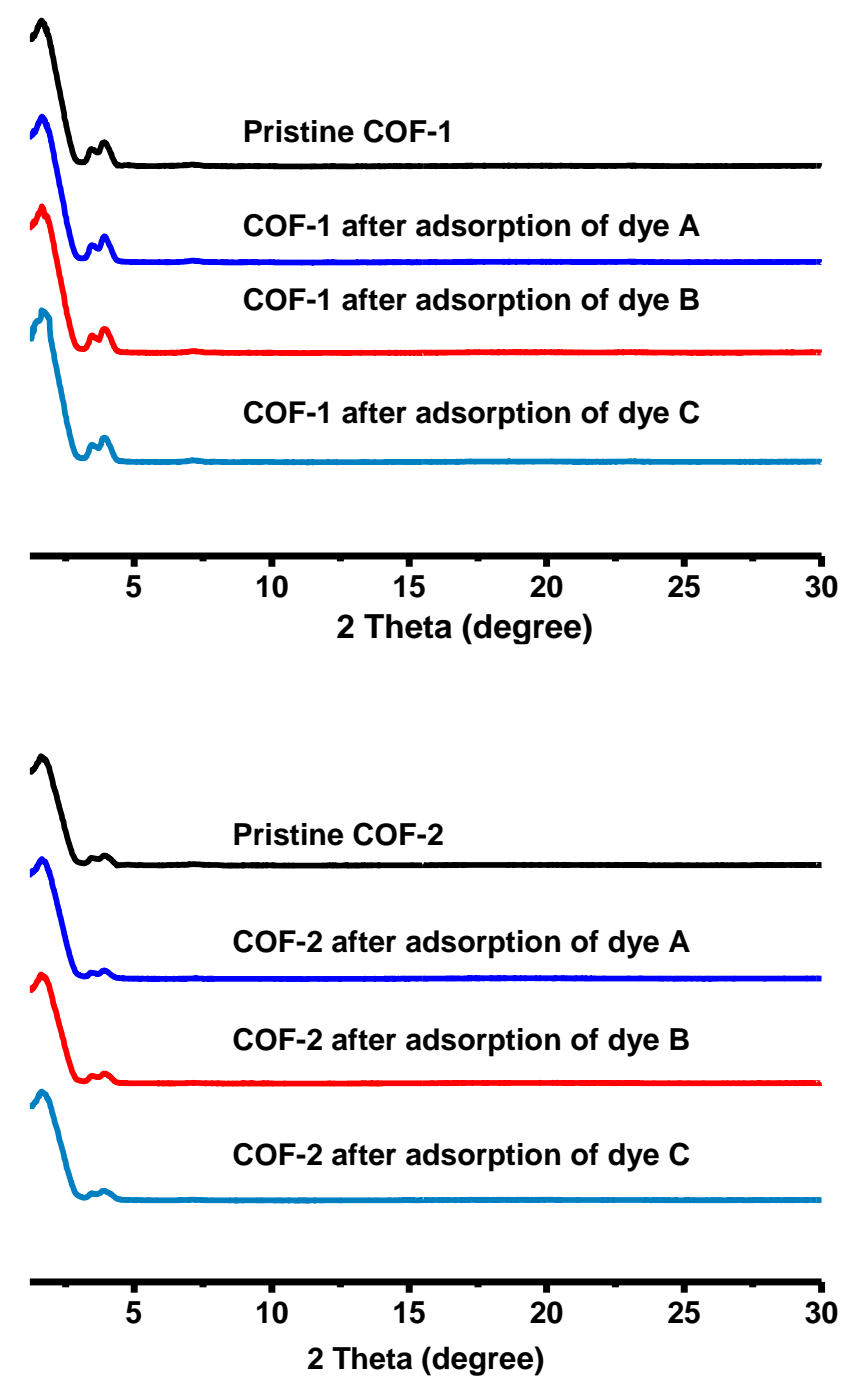

Figure S28 Adsorption curve of dye A after the recycle experiments

After recycle experiments, the as-obtained COFs were collected by filtration and washed with $\mathrm{MeOH}$ and $\mathrm{THF}$, and then dried at $60{ }^{\circ} \mathrm{C}$ under vacuum for $1 \mathrm{~h}$. Subsequently, the resulted COF-1 (5 mg) was soaked in EtOH solution of dye A for 24 h. To remove the dye adsorbed on the surface of COF-1, the resulted samples were washed with $\mathrm{EtOH}$ thoroughly until the washings became colorless. The washed samples were sonicated with solvent several times, and the solution of dye-A was diluted to $250 \mathrm{~mL}$. 


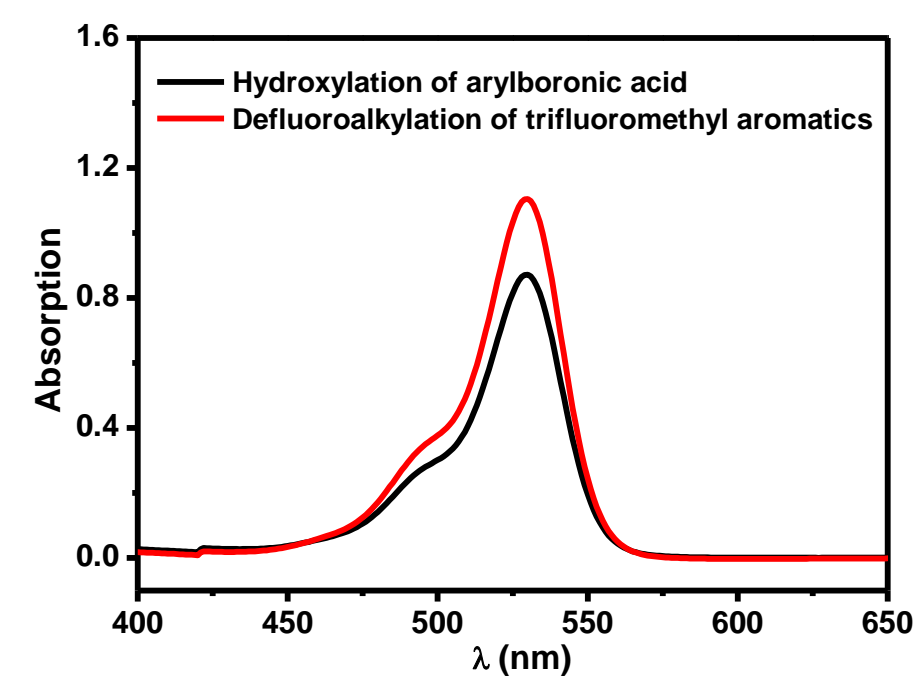

Figure S29 Uptake of dye A after the recycle experiments

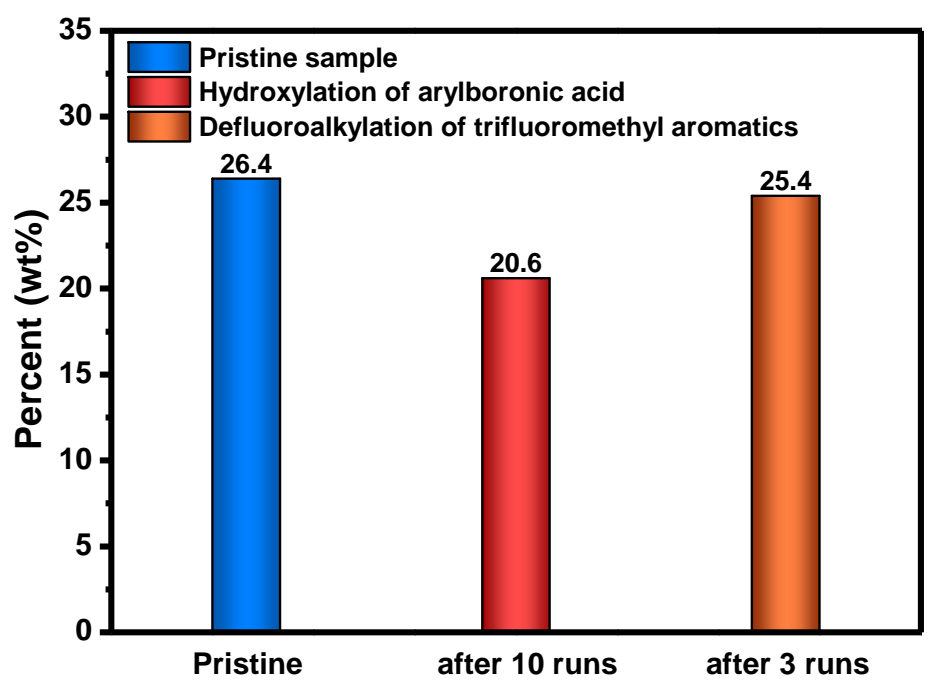

\section{Figure S30. ${ }^{1} \mathrm{H}$ NMR of digested COF-1}

The activated COF-1 $(20 \mathrm{mg}), \mathrm{CHCl}_{3}(30 \mathrm{~mL})$ and concentrated hydrochloric acid $(30 \mathrm{~mL})$ were added into the round-bottom flask. The mixture was heated at $50{ }^{\circ} \mathrm{C}$ for $12 \mathrm{~h}$ till a purple black clear solution was obtained. Then, the mixture was neutralized by $2 \mathrm{M} \mathrm{NaOH}(\mathrm{aq})$ and extracted with $\mathrm{CH}_{3} \mathrm{Cl}$. The organic phase was dried over anhydrous $\mathrm{Na}_{2} \mathrm{SO}_{4}$ and then concentrated under reduced pressure to remove the solvent. The mixture was dissolved into $\mathrm{CD}_{3} \mathrm{Cl}$ and ${ }^{1} \mathrm{H}-\mathrm{NMR}$ spectra were recorded. 
Figure S30. ${ }^{1} \mathrm{H}$ NMR of digested COF-1
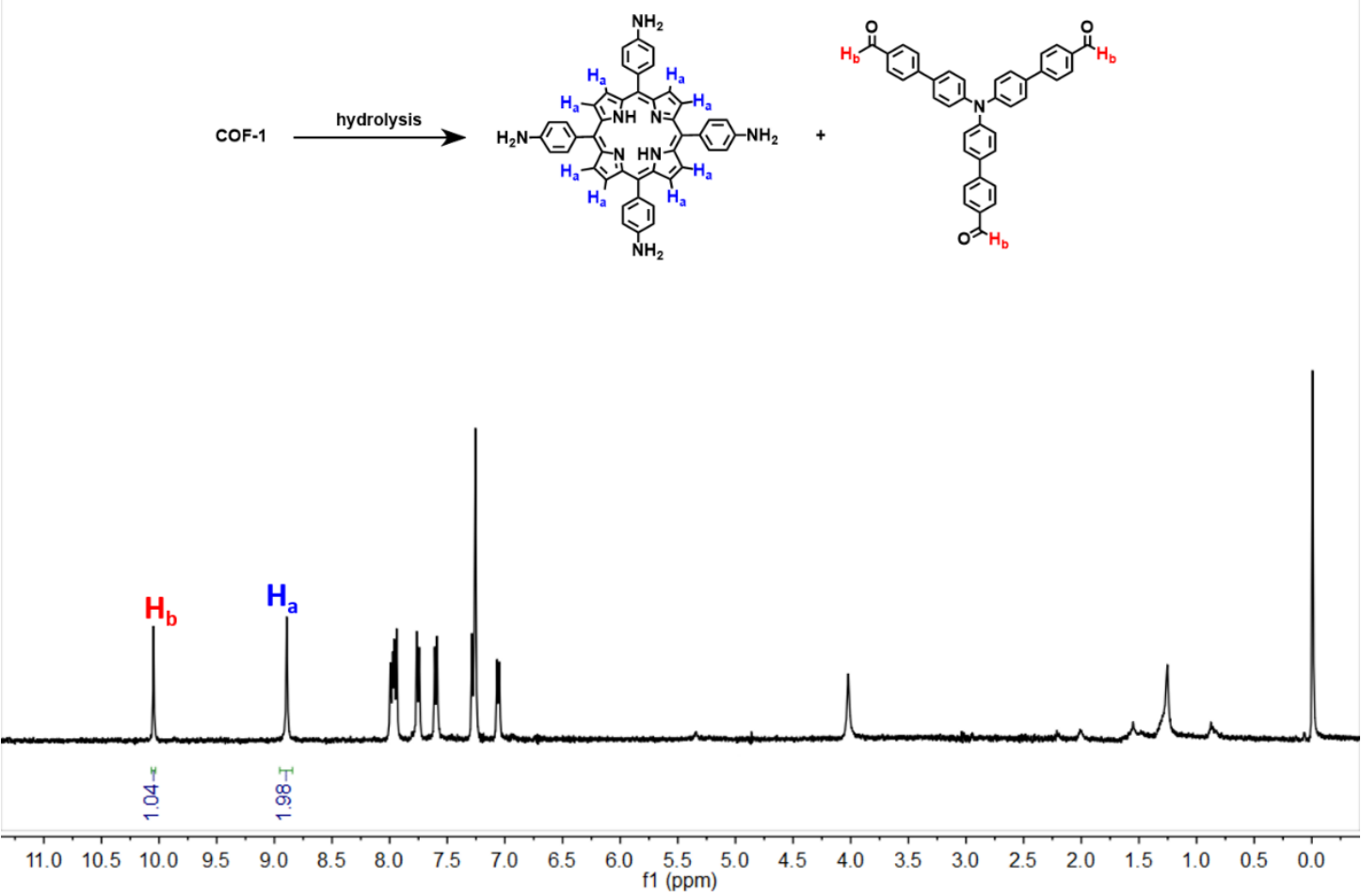

${ }^{1} \mathrm{H}$ NMR of digested COF-1 exhibited that the ratio of proton integration was 2:1 between $\mathrm{H}_{\mathrm{a}}$ of $\mathbf{H}_{2}$ (TAP) and $\mathrm{H}_{\mathrm{b}}$ of $\mathbf{N B C}$. Therefore, the ratio between $\mathrm{H}_{2}$ (TAP) and NBC was 3:4 in as-synthesized COF-1.

\section{Figure S31 a) Solid-state UV spectra and b) band gaps of monomers}

a)

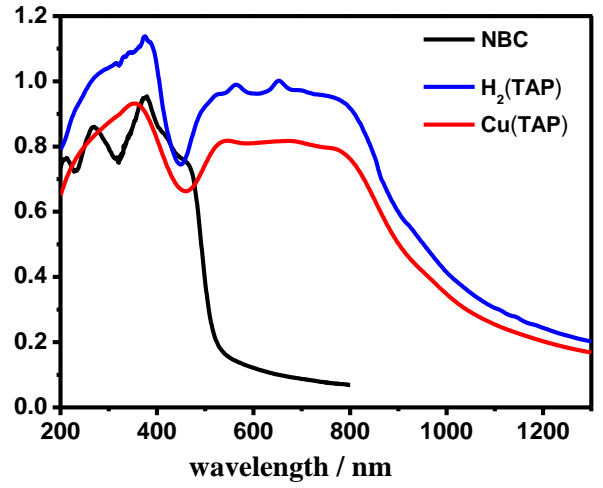

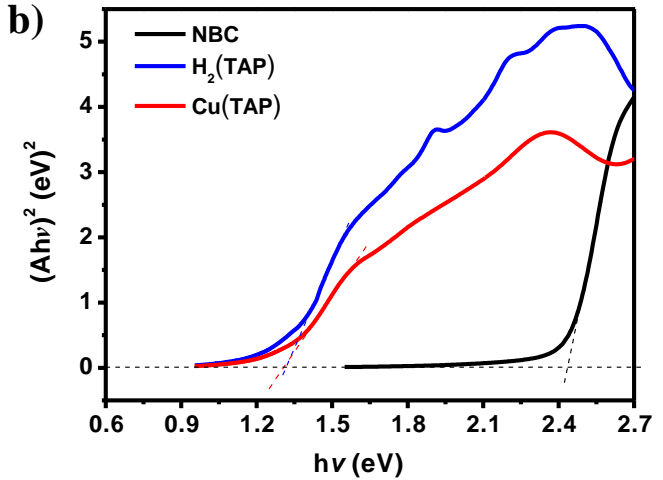




\section{Figure S32 Normalized absorption (black line) and emission spectra (red line)}

of COFs
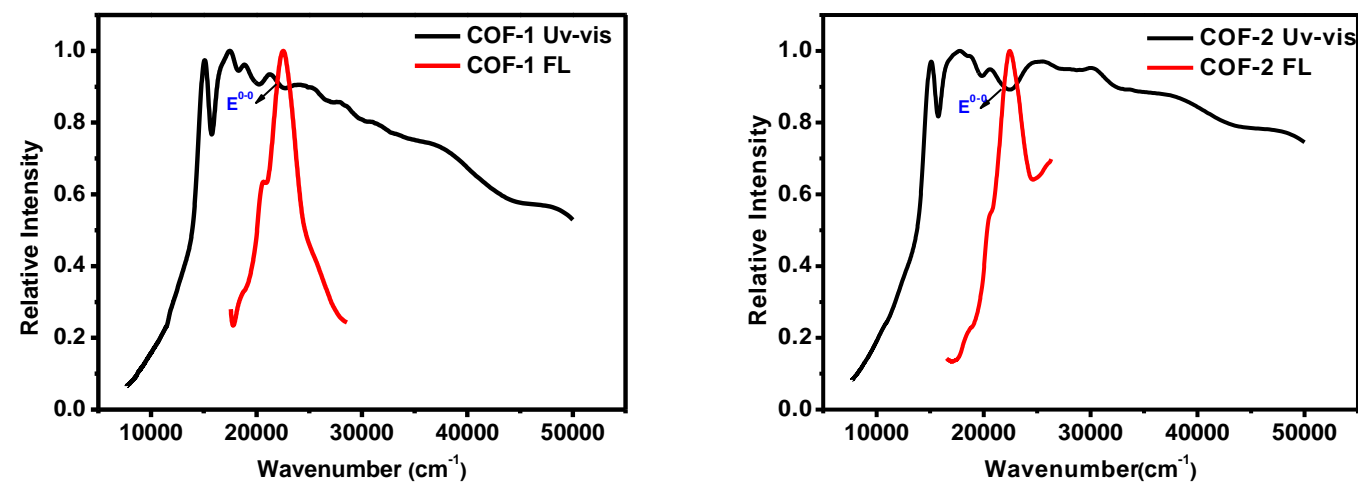

\section{Additional results of photocatalysis}

\subsection{Hydroxylation of arylboronic acids}

Table S18. Optimization of reaction conditions ${ }^{a}$

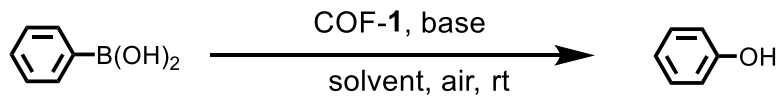

$4 a$

$5 \mathbf{a}$

\begin{tabular}{|c|c|c|c|c|}
\hline entry & solvent & base & loading of COF-1 & yield [ $\%$ ] \\
\hline 1 & $\mathrm{THF} / \mathrm{H}_{2} \mathrm{O}(4: 1, \mathrm{v} / \mathrm{v})$ & $\mathrm{Et}_{3} \mathrm{~N}$ & $1 \mathrm{~mol} \%$ & 65 \\
\hline 2 & $\mathrm{THF} / \mathrm{H}_{2} \mathrm{O}(4: 1, \mathrm{v} / \mathrm{v})$ & 2,6-lutidine & $1 \mathrm{~mol} \%$ & 57 \\
\hline 3 & $\mathrm{THF} / \mathrm{H}_{2} \mathrm{O}(4: 1, \mathrm{v} / \mathrm{v})$ & $i-\mathrm{Pr}_{2} \mathrm{NEt}$ & $1 \mathrm{~mol} \%$ & 71 \\
\hline 4 & $\mathrm{THF} / \mathrm{H}_{2} \mathrm{O}(4: 1, \mathrm{v} / \mathrm{v})$ & $i-\mathrm{Pr}_{2} \mathrm{NEt}$ & $5 \mathrm{~mol} \%$ & 85 \\
\hline 5 & $\mathrm{CH}_{3} \mathrm{CN} / \mathrm{H}_{2} \mathrm{O}(4: 1, \mathrm{v} / \mathrm{v})$ & $i-\mathrm{Pr}_{2} \mathrm{NEt}$ & $5 \mathrm{~mol} \%$ & 91 \\
\hline 6 & $\mathrm{DCM} / \mathrm{H}_{2} \mathrm{O}(4: 1, \mathrm{v} / \mathrm{v})$ & $i-\mathrm{Pr}_{2} \mathrm{NEt}$ & $5 \mathrm{~mol} \%$ & 83 \\
\hline 7 & $\mathrm{DMF} / \mathrm{H}_{2} \mathrm{O}(4: 1, \mathrm{v} / \mathrm{v})$ & $i-\mathrm{Pr}_{2} \mathrm{NEt}$ & $5 \mathrm{~mol} \%$ & 88 \\
\hline 8 & $\mathrm{MeOH} / \mathrm{H}_{2} \mathrm{O}(4: 1, \mathrm{v} / \mathrm{v})$ & $i-\mathrm{Pr}_{2} \mathrm{NEt}$ & $5 \mathrm{~mol} \%$ & 80 \\
\hline 9 & Toluene $/ \mathrm{H}_{2} \mathrm{O}(4: 1, \mathrm{v} / \mathrm{v})$ & $i-\mathrm{Pr}_{2} \mathrm{NEt}$ & $5 \mathrm{~mol} \%$ & 65 \\
\hline $10^{c}$ & $\mathrm{CH}_{3} \mathrm{CN} / \mathrm{H}_{2} \mathrm{O}(4: 1, \mathrm{v} / \mathrm{v})$ & $i-\mathrm{Pr}_{2} \mathrm{NEt}$ & $5 \mathrm{~mol} \%$ & trace \\
\hline $11^{d}$ & $\mathrm{CH}_{3} \mathrm{CN} / \mathrm{H}_{2} \mathrm{O}(4: 1, \mathrm{v} / \mathrm{v})$ & $i-\mathrm{Pr}_{2} \mathrm{NEt}$ & $5 \mathrm{~mol} \%$ & $<5$ \\
\hline $12^{\mathrm{e}}$ & $\mathrm{CH}_{3} \mathrm{CN} / \mathrm{H}_{2} \mathrm{O}(4: 1, \mathrm{v} / \mathrm{v})$ & $i-\mathrm{Pr}_{2} \mathrm{NEt}$ & $5 \mathrm{~mol} \%$ & 0 \\
\hline
\end{tabular}

${ }^{a}$ Reaction conditions: $4 \mathbf{a}(0.5 \mathrm{mmol})$, base $(1.5 \mathrm{mmol})$, COF-1, solvent $(3 \mathrm{~mL})$ under air atmosphere by LED for $30 \mathrm{~h} .{ }^{b}$ Isolated yields after column chromatography. ${ }^{c} \mathrm{~N}_{2}$ instead of $\mathrm{O}_{2} \cdot{ }^{d}$ in the dark. ${ }^{e}$ Without COF-1. 
Table S19. Results of recycle experiment

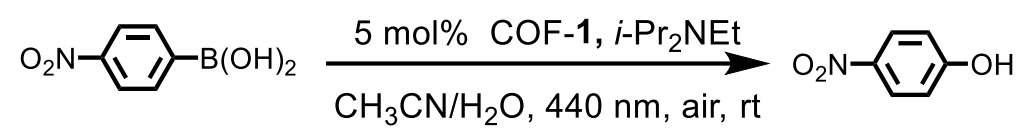

\begin{tabular}{cc}
\hline run & yield $(\%)$ \\
\hline 1 & 99 \\
2 & 98 \\
3 & 99 \\
4 & 97 \\
5 & 98 \\
6 & 96 \\
7 & 97 \\
8 & 95 \\
9 & 96 \\
10 & 97 \\
\hline
\end{tabular}

Figure S33. Quenching experiments using TEMP and DMPO

a)

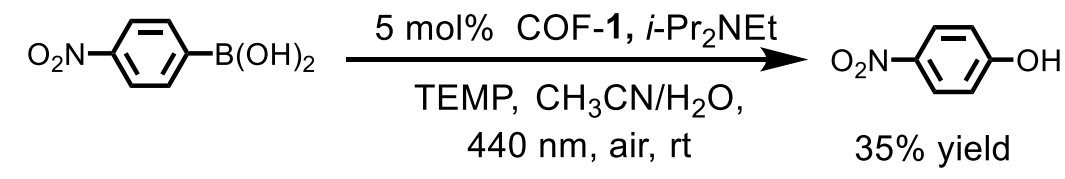

b) $\mathrm{O}_{2} \mathrm{~N}-\mathrm{B}(\mathrm{OH})_{2}$

$$
\underset{\substack{\mathrm{DMPO}, \mathrm{CH}_{3} \mathrm{CN} / \mathrm{H}_{2} \mathrm{O} \\ 440 \mathrm{~nm} \text {, air, } \mathrm{rt}}}{5 \mathrm{~mol} \% \mathrm{COF}-1, i-\mathrm{Pr}_{2} \mathrm{NEt}} \mathrm{O}_{2} \mathrm{~N} \underset{2}{2}
$$

Figure S34. HRMS data of 4-nitrophenol for the catalytic reaction in the presence of $\mathrm{H}_{2}{ }^{18} \mathrm{O}$.

The data indicated that the product was not labeled by ${ }^{18} \mathrm{O}$

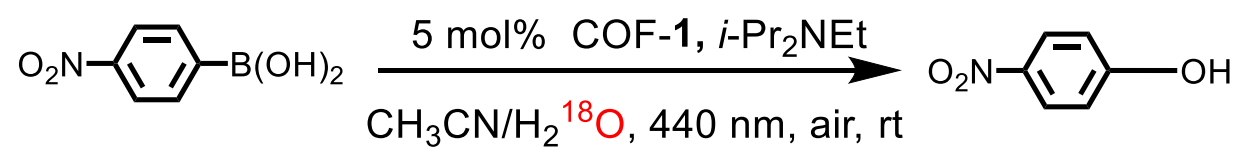




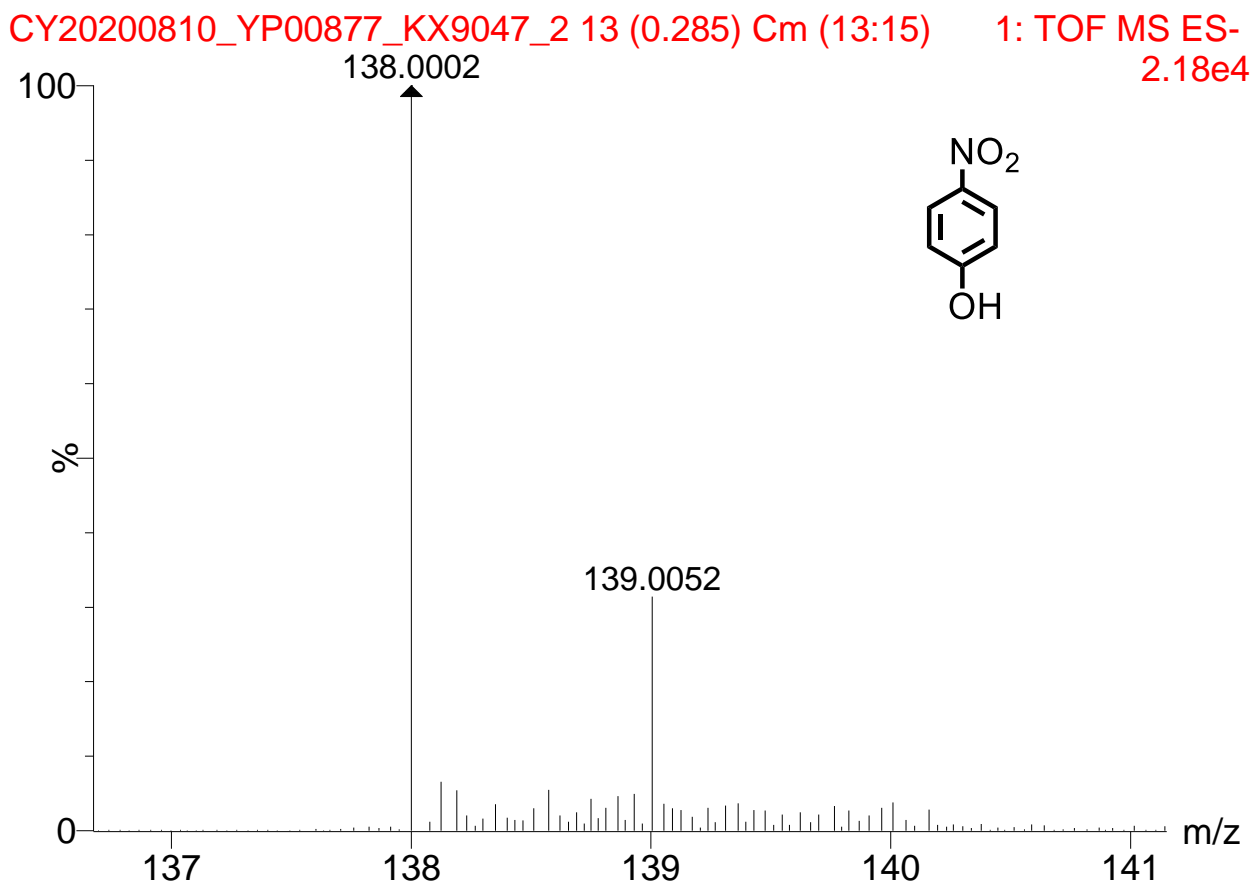

Figure S35. NMR of 4-nitrophenol for the reaction in the presence of $\mathrm{D}_{2} \mathrm{O}$.

The datas indicated that the product was labeled by $\mathrm{D}$

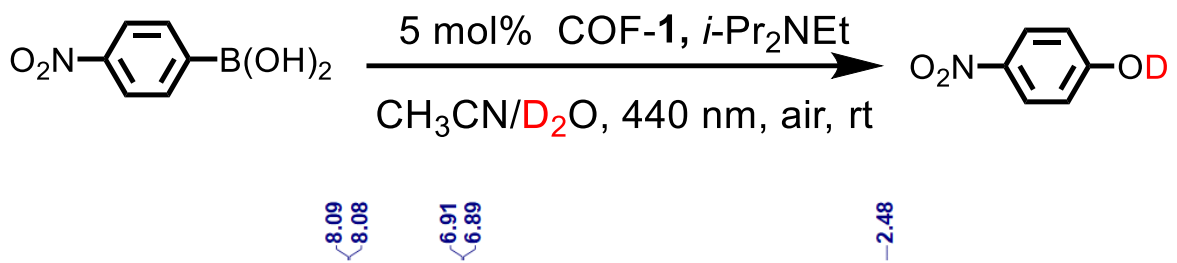

1.

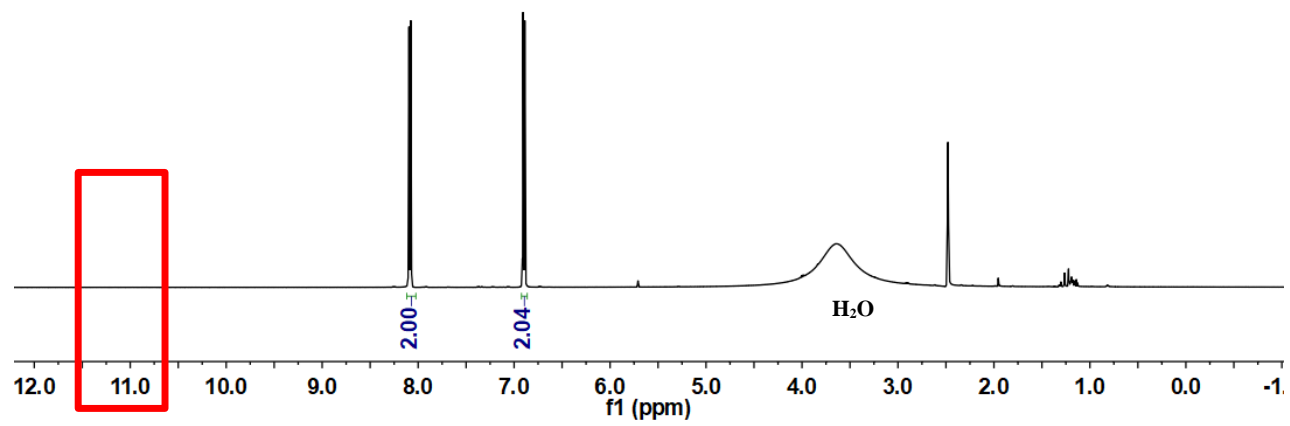


Figure S36. Proposed reaction mechanism

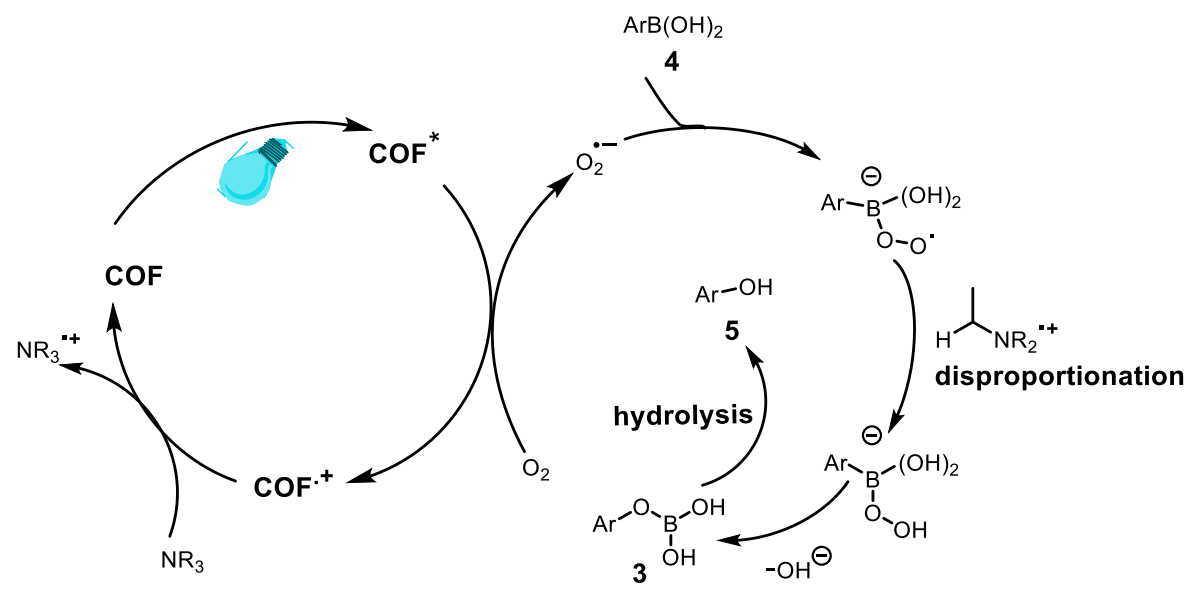

\subsection{Defluoroalkylation of 1,3-bis(trifluoromethyl)benzene}

Table S20. Optimization of reaction conditions ${ }^{a}$

\begin{tabular}{|c|c|c|c|c|}
\hline$Y_{1}$ & $\widehat{N a H}^{\mathrm{OH}}$ & $\begin{array}{l}\text { 1, CySH } \\
\text { Ivent, } 440 \mathrm{~nm} \\
\mathrm{~N}_{2}, 30 \mathrm{~h}\end{array}$ & 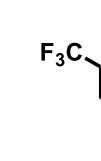 & \\
\hline entry & solvent & base & temp. & yield [ \% ] \\
\hline 1 & $\mathrm{H}_{2} \mathrm{O} / \mathrm{DMSO}(1: 20, \mathrm{v} / \mathrm{v})$ & $\mathrm{HCOONa}$ & $25^{\circ} \mathrm{C}$ & 48 \\
\hline 2 & $\mathrm{H}_{2} \mathrm{O} / \mathrm{DMSO}(1: 20, \mathrm{v} / \mathrm{v})$ & HcoOcs & $25^{\circ} \mathrm{C}$ & 45 \\
\hline 3 & $\mathrm{H}_{2} \mathrm{O} / \mathrm{DMSO}(1: 20, \mathrm{v} / \mathrm{v})$ & $\mathrm{NaHCO}_{3}$ & $25^{\circ} \mathrm{C}$ & 16 \\
\hline 4 & $\mathrm{H}_{2} \mathrm{O} / \mathrm{DMSO}(1: 20, \mathrm{v} / \mathrm{v})$ & $i-\mathrm{Pr}_{2} \mathrm{NEt}$ & $25^{\circ} \mathrm{C}$ & no detected \\
\hline 5 & $\mathrm{H}_{2} \mathrm{O} / \mathrm{DMSO}(1: 20, \mathrm{v} / \mathrm{v})$ & 2,6-lutidine & $25^{\circ} \mathrm{C}$ & no detected \\
\hline 6 & $\mathrm{H}_{2} \mathrm{O} / \mathrm{CH}_{3} \mathrm{CN}(1: 20, \mathrm{v} / \mathrm{v})$ & HCOONa & $25^{\circ} \mathrm{C}$ & 35 \\
\hline 7 & $\mathrm{H}_{2} \mathrm{O} / \mathrm{DMF}(1: 20, \mathrm{v} / \mathrm{v})$ & HCOONa & $25^{\circ} \mathrm{C}$ & 38 \\
\hline 8 & $\mathrm{H}_{2} \mathrm{O} / \mathrm{THF}(1: 20, \mathrm{v} / \mathrm{v})$ & HCOONa & $25^{\circ} \mathrm{C}$ & 43 \\
\hline 9 & $\mathrm{H}_{2} \mathrm{O} / \mathrm{DMSO}(1: 20, \mathrm{v} / \mathrm{v})$ & HCOONa & $40^{\circ} \mathrm{C}$ & 69 \\
\hline 10 & $\mathrm{H}_{2} \mathrm{O} / \mathrm{DMSO}(1: 20, \mathrm{v} / \mathrm{v})$ & $\mathrm{HCOONa}$ & $60^{\circ} \mathrm{C}$ & 83 \\
\hline 11 & $\mathrm{H}_{2} \mathrm{O} / \mathrm{DMSO}(1: 20, \mathrm{v} / \mathrm{v})$ & $\mathrm{HCOONa}$ & $80^{\circ} \mathrm{C}$ & 75 \\
\hline $12^{c}$ & $\mathrm{H}_{2} \mathrm{O} / \mathrm{DMSO}(1: 20, \mathrm{v} / \mathrm{v})$ & $\mathrm{HCOONa}$ & $60^{\circ} \mathrm{C}$ & 45 \\
\hline $13^{d}$ & $\mathrm{H}_{2} \mathrm{O} / \mathrm{DMSO}(1: 20, \mathrm{v} / \mathrm{v})$ & $\mathrm{HCOONa}$ & $60^{\circ} \mathrm{C}$ & no detected \\
\hline 14 & $\mathrm{H}_{2} \mathrm{O} / \mathrm{DMSO}(1: 20, \mathrm{v} / \mathrm{v})$ & Without & $60^{\circ} \mathrm{C}$ & no detected \\
\hline $15^{\mathrm{e}}$ & $\mathrm{H}_{2} \mathrm{O} / \mathrm{DMSO}(1: 20, \mathrm{v} / \mathrm{v})$ & HCOONa & $60^{\circ} \mathrm{C}$ & no detected \\
\hline $16^{f}$ & $\mathrm{H}_{2} \mathrm{O} / \mathrm{DMSO}(1: 20, \mathrm{v} / \mathrm{v})$ & HCOONa & $60^{\circ} \mathrm{C}$ & no detected \\
\hline
\end{tabular}

${ }^{a}$ Reaction conditions: 6 (0.5 mmol), 7a (1.5 mmol), COF-1 (0.025 mmol), CySH (0.05 mmol) in $5 \mathrm{~mL}$ of solvent under $\mathrm{N}_{2} .{ }^{b}$ Isolated yield. ${ }^{c} \mathrm{O}_{2}$ instead of $\mathrm{N}_{2} .{ }^{d}$ Without COF1 as an photocatalyst. ${ }^{e}$ in the dark. ${ }^{f}$ Without CySH. 
Table S21. Results of recycle experiment

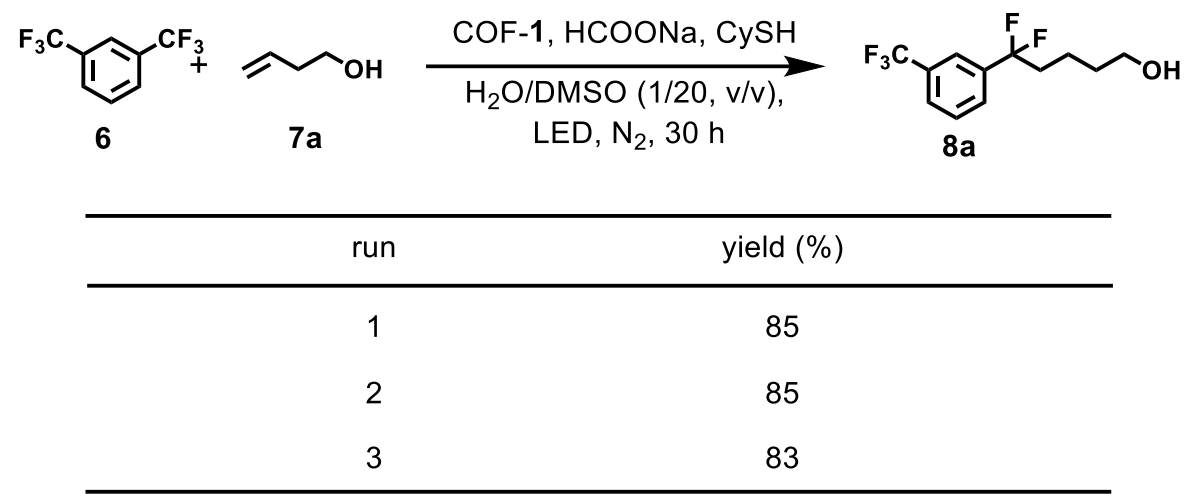

Figure S37. PXRD of COF-1 after three runs

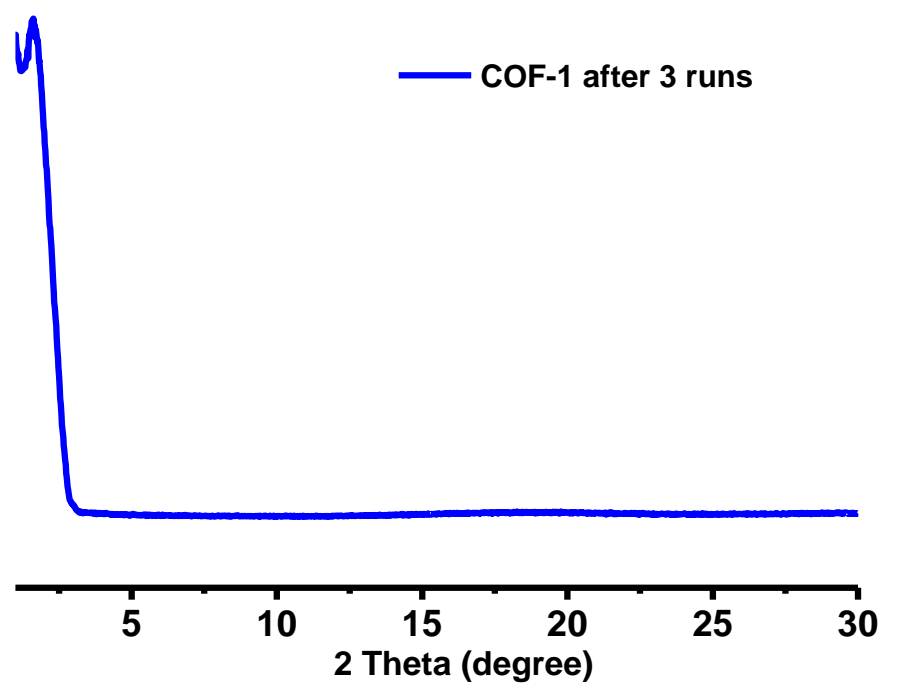

Figure S38 Stern-Volmer quenching experiment of COF-1

To understand the role of COFs in the defluoroalkylation, Stern-Volmer quenching experiments of COF-1 were conducted in $\mathrm{H}_{2} \mathrm{O} / \mathrm{DMSO}(1 / 20$, v/v). COF-1 excited at $450 \mathrm{~nm}$ in a quartz cuvette. The emission of COF-1 was measured by the addition of $\mathbf{6}$, 7a, cyclohexanethiol, and sodium formate, however the fluorescence intensity of COF$\mathbf{1}$ decreased only with increasing amounts of $\mathbf{6}$, which is the evidence for the direct substrate reduction by COFs (COFs*). 


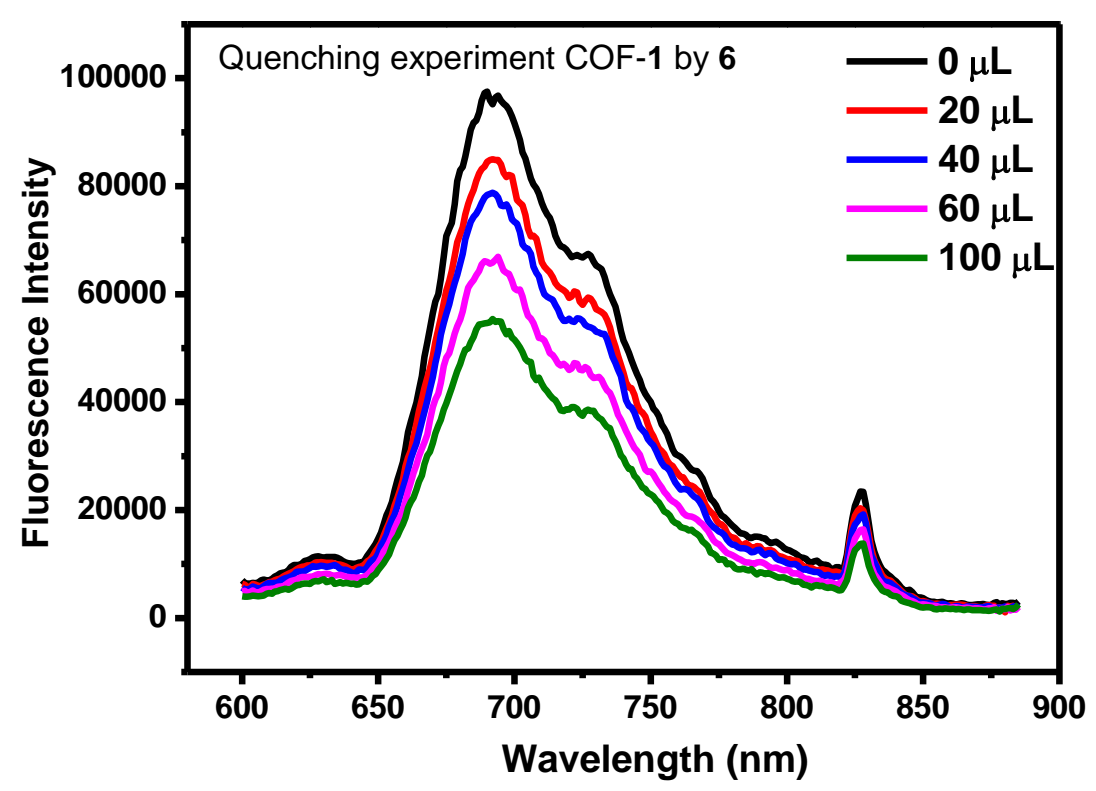

Table S22. Thiol loading experiment for the defluoroalkylation of 1,3bis(trifluoromethyl)benzene<smiles>FC(F)(F)c1cccc(C(F)(F)F)c1</smiles>

6

$+$<smiles>C=CCCO</smiles>

$7 a$<smiles>OCCCCC(F)(F)c1cccc(C(F)(F)F)c1</smiles>

$8 a$

$+$<smiles>FC(F)(F)c1cccc(C(F)(F)F)c1</smiles>

12

Thiol loading

yield (8a)

yield (12)

\begin{tabular}{lll}
\hline 5 mol\% & $85 \%$ & $5 \%$ \\
$50 \mathrm{~mol} \%$ & $47 \%$ & $31 \%$ \\
$150 \mathrm{~mol} \%$ & $11 \%$ & $54 \%$ \\
\hline
\end{tabular}


Figure S39. Proposed reaction mechanism

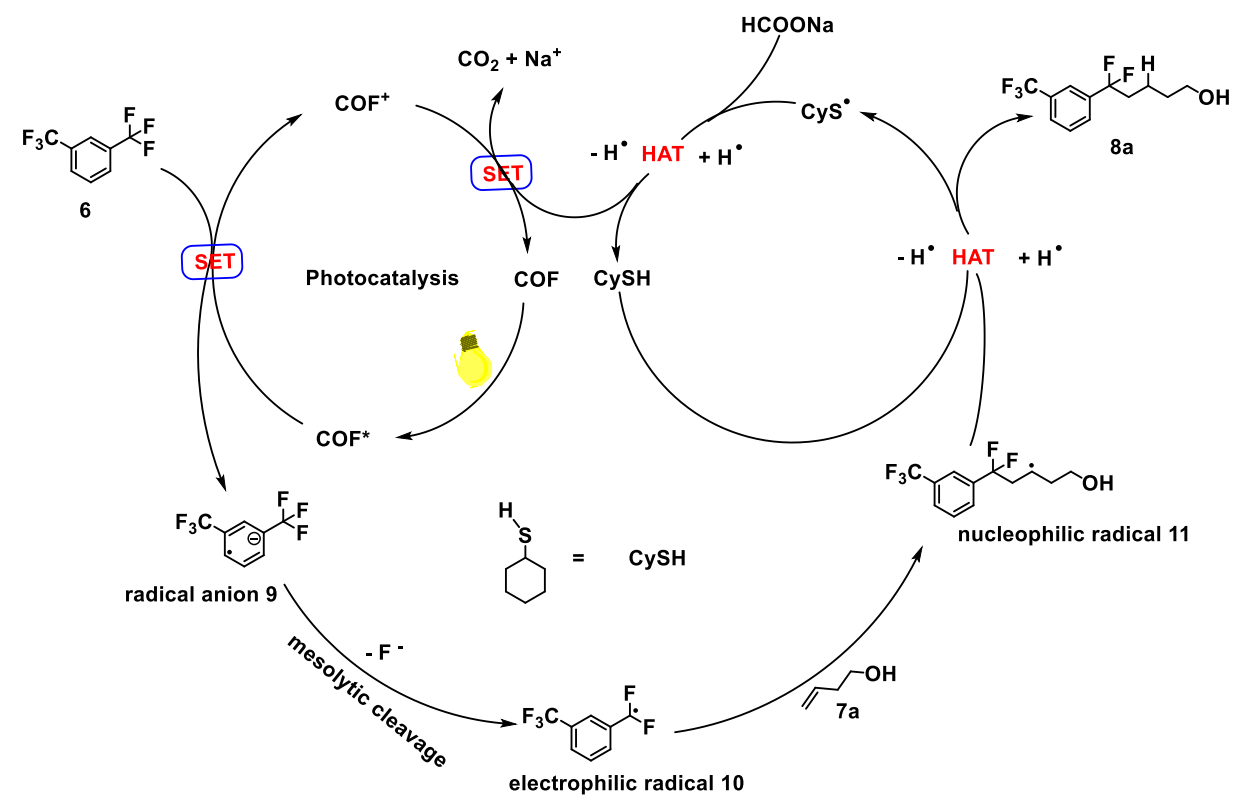

Scheme S1. Control experiment using NBC, $\mathrm{H}_{2}(\mathrm{TAP})$ and $\mathrm{Cu}(\mathrm{TAP})$ as photocatalysts

a)<smiles>O=[N+]([O-])c1ccc(Br)cc1</smiles>

b) $\mathrm{O}_{2} \mathrm{~N}-\mathrm{B}(\mathrm{OH})_{2}$ $4 \mathrm{c}$

c)

$$
{ }_{4 c}
$$
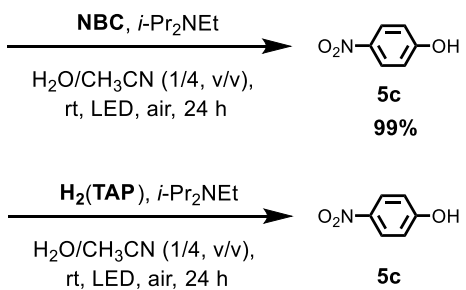
$\mathrm{rt}, \mathrm{LED}$, air, $24 \mathrm{~h}$

$$
\underset{\substack{\mathrm{H}_{2} \mathrm{O} / \mathrm{CH}_{3} \mathrm{CN}(1 / 4, \mathrm{v} / \mathrm{v}), \mathrm{rt}, \mathrm{LED}, \text { air, } 24 \mathrm{~h}}}{\stackrel{\mathrm{Cu}(\mathrm{TAP}), i-\mathrm{Pr}_{2} \mathrm{NEt}}{\longrightarrow}}
$$
rt, LED, air, $24 \mathrm{~h}$

d)

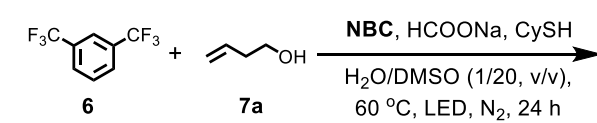

$7 a$

$60{ }^{\circ} \mathrm{C}$, LED, $\mathrm{N}_{2}, 24 \mathrm{~h}$

e) ${ }_{6}^{\mathrm{F}_{3} \mathrm{C}} \mathrm{Y}^{\mathrm{CF}_{3}}+$

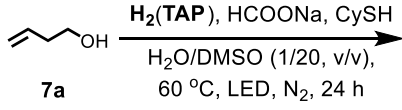

f) $\underbrace{\mathrm{F}_{3} \mathrm{C}}_{6} \underbrace{\mathrm{CF}_{3}}_{\mathrm{7a}}+\underbrace{\frac{\mathrm{Cu}(\mathrm{TAP}), \mathrm{HCOONa}, \mathrm{CySH}}{\longrightarrow}}_{\begin{array}{l}\mathrm{H}_{2} \mathrm{O} / \mathrm{DMSO}(1 / 20, \mathrm{v} / \mathrm{v}), \\ 60^{\circ} \mathrm{C}, \mathrm{LED}, \mathrm{N}_{2}, 24 \mathrm{~h}\end{array}}$

$$
\begin{gathered}
\mathrm{O}_{2} \mathrm{~N}-\mathrm{OH} \\
83 \%
\end{gathered}
$$<smiles>O=[N+]([O-])c1ccc(O)cc1</smiles>

$5 c$

$67 \%$

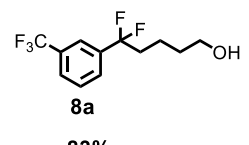<smiles>OCCCCC(F)(F)c1cccc(C(F)(F)F)c1</smiles>

$61 \%$

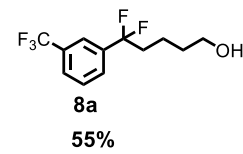


Scheme S2. Sterically substrates catalyzed by COF-1

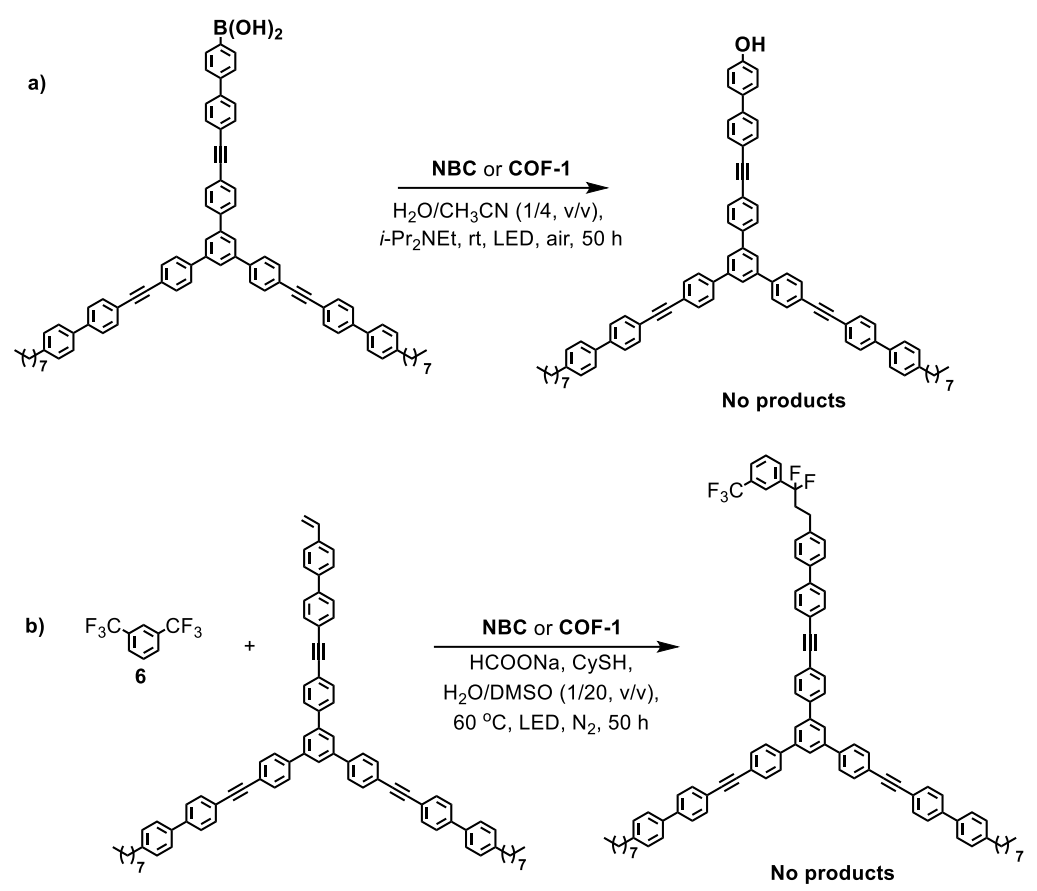

Scheme S3. Control experiment using dye B and dye B @COF-1 as photocatalysts in the hydroxylation of biphenylboric acid

(a) The activated COF-1 was soaked in a EtOH solution of biphenylboric acid (4f) for $24 \mathrm{~h}$. After removing the dyes adsorbed on the surface of COF-1, 4f @COF-1 was used for the hydroxylation.

(b) Dye $\mathbf{B}$ as a photocatalyst was used for the hydroxylation.

(c) The activated COF-1 was soaked in a THF solution of dye $\mathbf{B}$ for $24 \mathrm{~h}$. After removing the dyes adsorbed on the surface of COF-1, dye B @COF-1 was used for the hydroxylation. 
a)

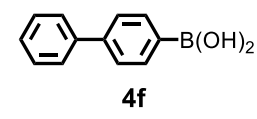

b)

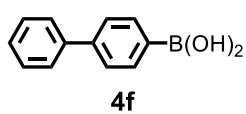

c)

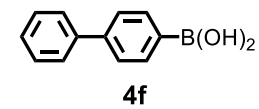

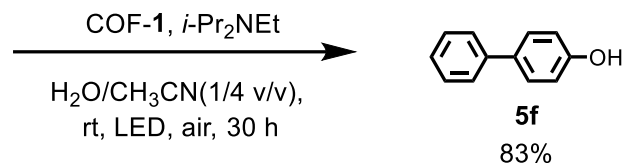

$\underset{\substack{\mathrm{H}_{2} \mathrm{O} / \mathrm{CH}_{3} \mathrm{CN}(1 / 4 \mathrm{v} / \mathrm{v}), \mathrm{rt}, \mathrm{LED}, \text { air, } 30 \mathrm{~h}}}{\text { Dye B, } i-\mathrm{Pr}_{2} \mathrm{NEt}}$

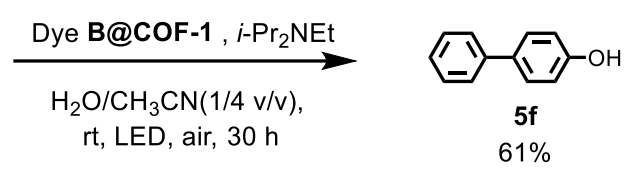

Table S23. Reported results of other kinds of photocatalysts in the hydroxylation of arylboronic acids

\begin{tabular}{|c|c|c|c|c|c|}
\hline \multirow{2}{*}{ Entry } & \multirow{2}{*}{ Photocatalysts } & \multicolumn{2}{|c|}{ Yield } & \multirow{2}{*}{ Reporters } & \multirow{2}{*}{ References } \\
\hline & & $5 \mathbf{a}$ & $5 c$ & & \\
\hline 1 & $\mathrm{Ru}(\mathrm{bpy})_{3} \mathrm{Cl}_{2}$ & $81 \%$ & - & $\begin{array}{c}\text { Wen-Jing Xiao } \\
\text { et. al. }\end{array}$ & $\begin{array}{c}\text { Angew. Chem. Int. Ed., } \\
\text { 2012, 51, 784-788 }\end{array}$ \\
\hline 2 & Methylene Blue & $94 \%$ & $98 \%$ & $\begin{array}{c}\text { Juan C. Scaiano } \\
\text { et. al. }\end{array}$ & $\begin{array}{c}\text { J. Am. Chem. Soc., } \\
\text { 2013, } 135,13286- \\
13289\end{array}$ \\
\hline 3 & $\begin{array}{c}\text { N-Substituted } \\
\text { 3(10H)- } \\
\text { Acridones }\end{array}$ & $97 \%$ & - & $\begin{array}{l}\text { Qing-Long } \mathrm{Xu} \\
\text { et. al. }\end{array}$ & $\begin{array}{c}\text { J. Org. Chem., } \\
\text { 2017, 82, 5236-5241 }\end{array}$ \\
\hline 4 & СРОР-29 & $98 \%$ & - & $\begin{array}{c}\text { Bao-Hang Han } \\
\text { et. al. }\end{array}$ & $\begin{array}{c}\text { ACS Catal., } \\
\text { 2018, 8, 5313-5322 }\end{array}$ \\
\hline 5 & $\mathrm{ZnPC}$ & $98 \%$ & $97 \%$ & $\begin{array}{c}\text { Hua-Yue Wu } \\
\text { et. al. }\end{array}$ & $\begin{array}{l}\text { Adv. Synth. Catal., } \\
\text { 2019, 361, 961-964 }\end{array}$ \\
\hline 6 & LZU-190 & $88 \%$ & $99 \%$ & $\begin{array}{c}\text { Wei Wang } \\
\text { et. al. }\end{array}$ & $\begin{array}{l}\text { J. Am. Chem. Soc., } \\
\text { 2018, 140, 4623-4631 }\end{array}$ \\
\hline
\end{tabular}




\begin{tabular}{|c|c|c|c|c|c|}
\hline $\mathbf{7}$ & Rose Bengal & $97 \%$ & $79 \%$ & $\begin{array}{c}\text { Bo Zhang } \\
\text { et. al. }\end{array}$ & $\begin{array}{c}\text { Org. Lett., } \\
\mathbf{2 0 1 8}, 20,4979-4983\end{array}$ \\
\hline $\mathbf{8}$ & $\mathrm{Cl}_{3} \mathrm{CCN}$ & $96 \%$ & $79 \%$ & $\begin{array}{c}\text { Lei Shi } \\
\text { et. al. }\end{array}$ & $\begin{array}{c}\text { Org. Biomol. } \text { Chem., } \\
\mathbf{2 0 1 9}, 17,7558-7563\end{array}$ \\
\hline $\mathbf{9}$ & thioxanthone & $94 \%$ & $95 \%$ & $\begin{array}{c}\text { Hao Gao } \\
\text { et. al. }\end{array}$ & $\begin{array}{c}\text { Tetrahedron Lett., } \\
\mathbf{2 0 1 9}, 60,660-663\end{array}$ \\
\hline
\end{tabular}

16 NMR results of photocatalysis

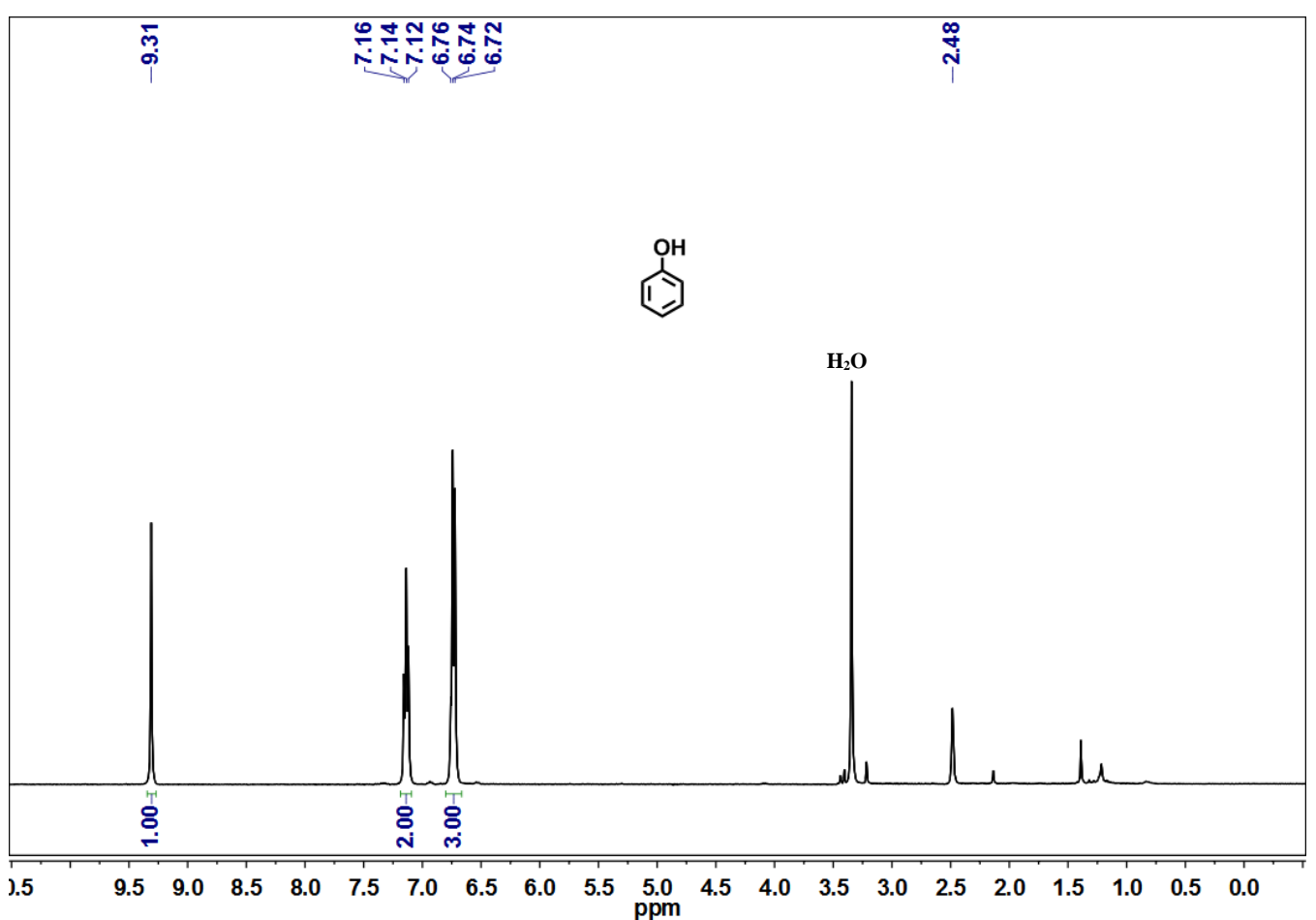

Phenol (5a) ${ }^{1} \mathrm{H}$ NMR (400 MHz, DMSO- $\left.d_{6}\right) \delta 9.31(\mathrm{~s}, 1 \mathrm{H}), 7.16-7.12(\mathrm{t}, 2 \mathrm{H}), 6.76-6.72$ $(\mathrm{t}, 3 \mathrm{H})$. 


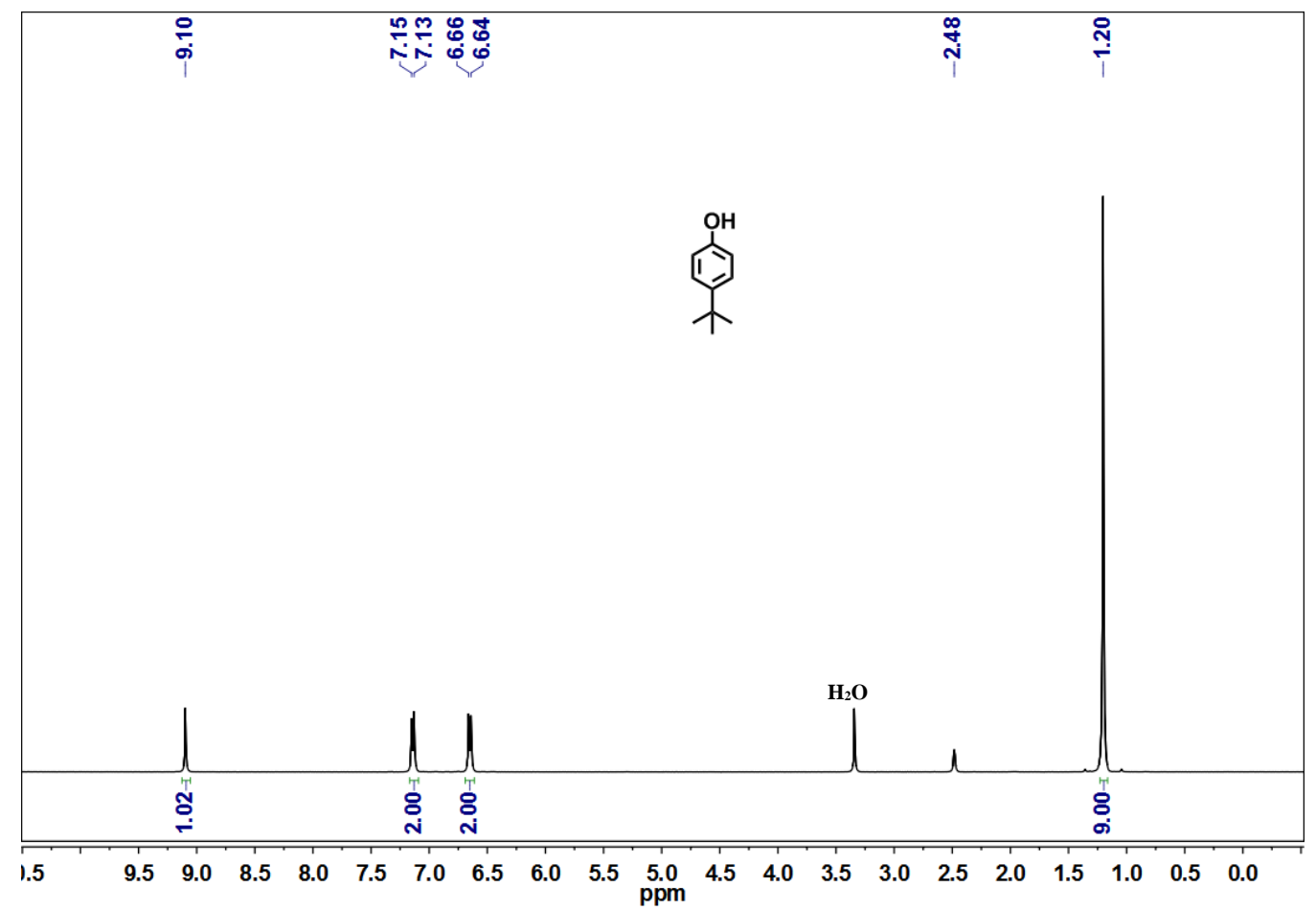

4-(tert-butyl)phenol (5b) ${ }^{1} \mathrm{H}$ NMR (400 MHz, DMSO-d $\left.)\right) \delta 9.10(\mathrm{~s}, 1 \mathrm{H}), 7.15-7.13$ (d, $J=8.4 \mathrm{~Hz}, 2 \mathrm{H}), 6.66-6.64(\mathrm{~d}, J=8.4 \mathrm{~Hz}, 2 \mathrm{H}), 1.20(\mathrm{~s}, 9 \mathrm{H})$.

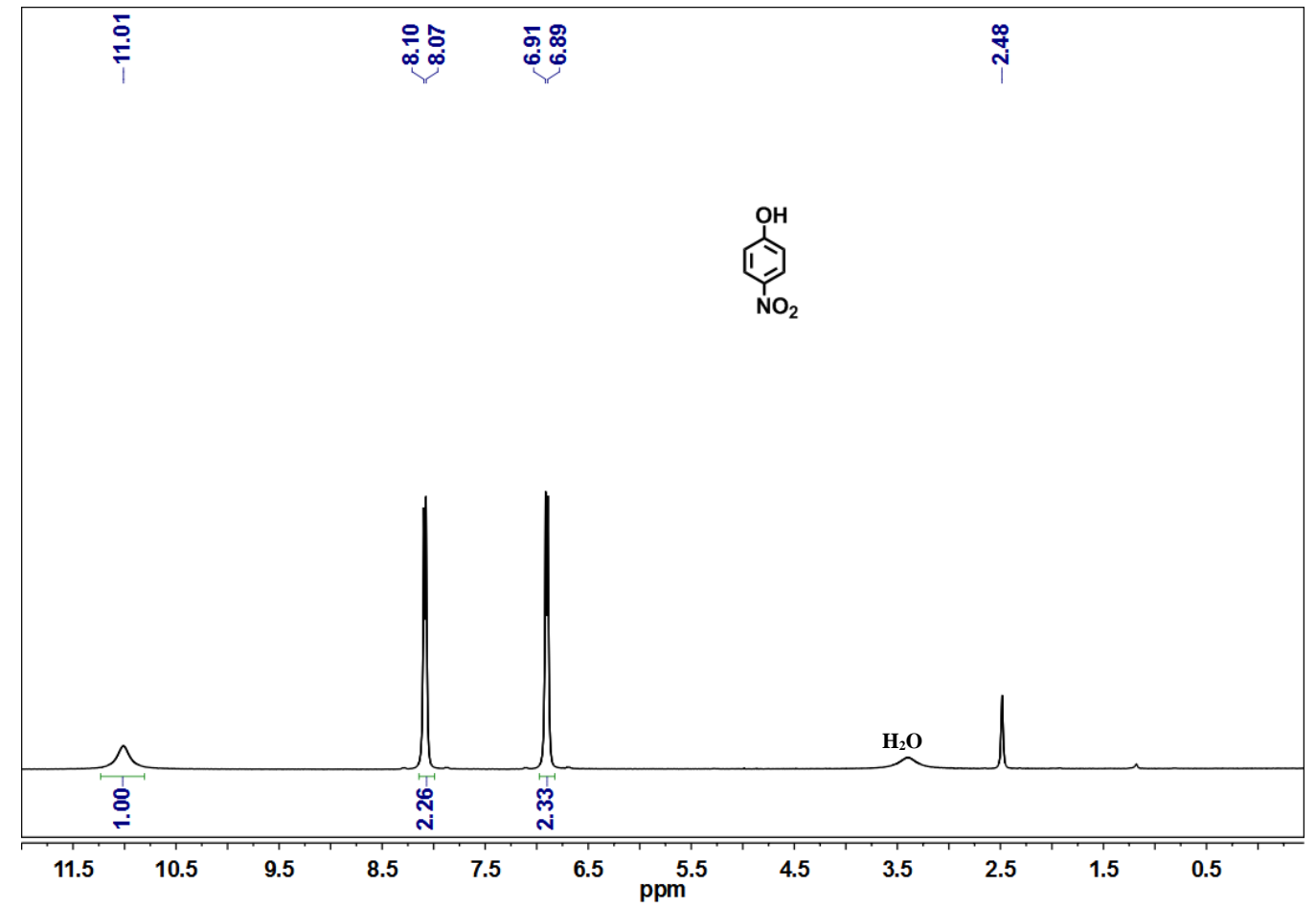

4-nitrophenol (5c) ${ }^{1} \mathrm{H}$ NMR (400 MHz, DMSO-d $) \delta 11.01(\mathrm{~s}, 1 \mathrm{H}), 8.10-8.07$ (d, $J=$ $8.6 \mathrm{~Hz}, 2 \mathrm{H}), 6.91-6.89$ (d, $J=8.6 \mathrm{~Hz}, 2 \mathrm{H})$. 


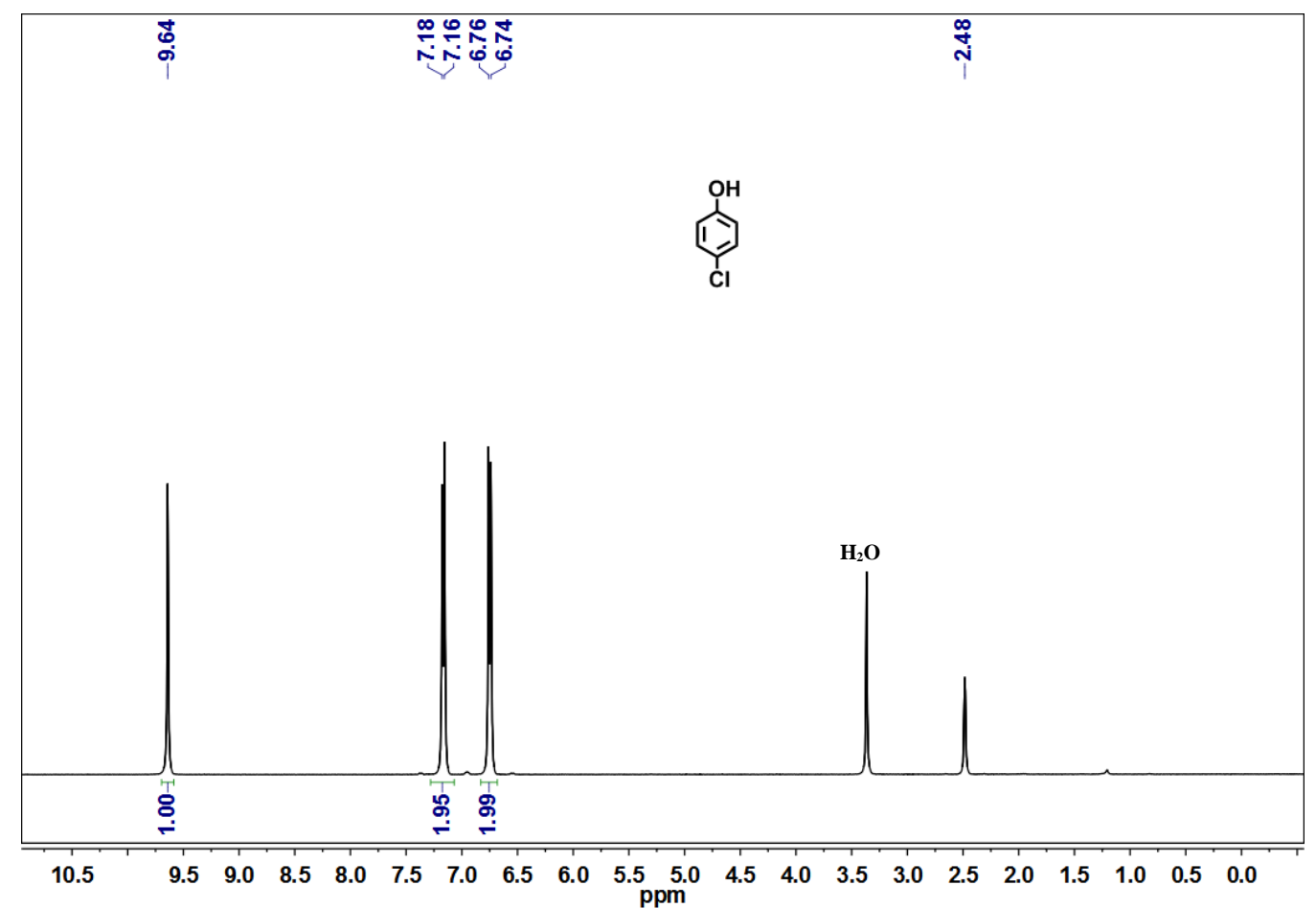

4-chlorophenol (5d) ${ }^{1} \mathrm{H}$ NMR (400 MHz, DMSO- $\left.d_{6}\right) \delta 9.64$ (s, 1H), 7.18-7.16 (d, $J=$ $8.4 \mathrm{~Hz}, 2 \mathrm{H}), 6.76-6.74(\mathrm{~d}, J=8.4 \mathrm{~Hz}, 2 \mathrm{H})$.

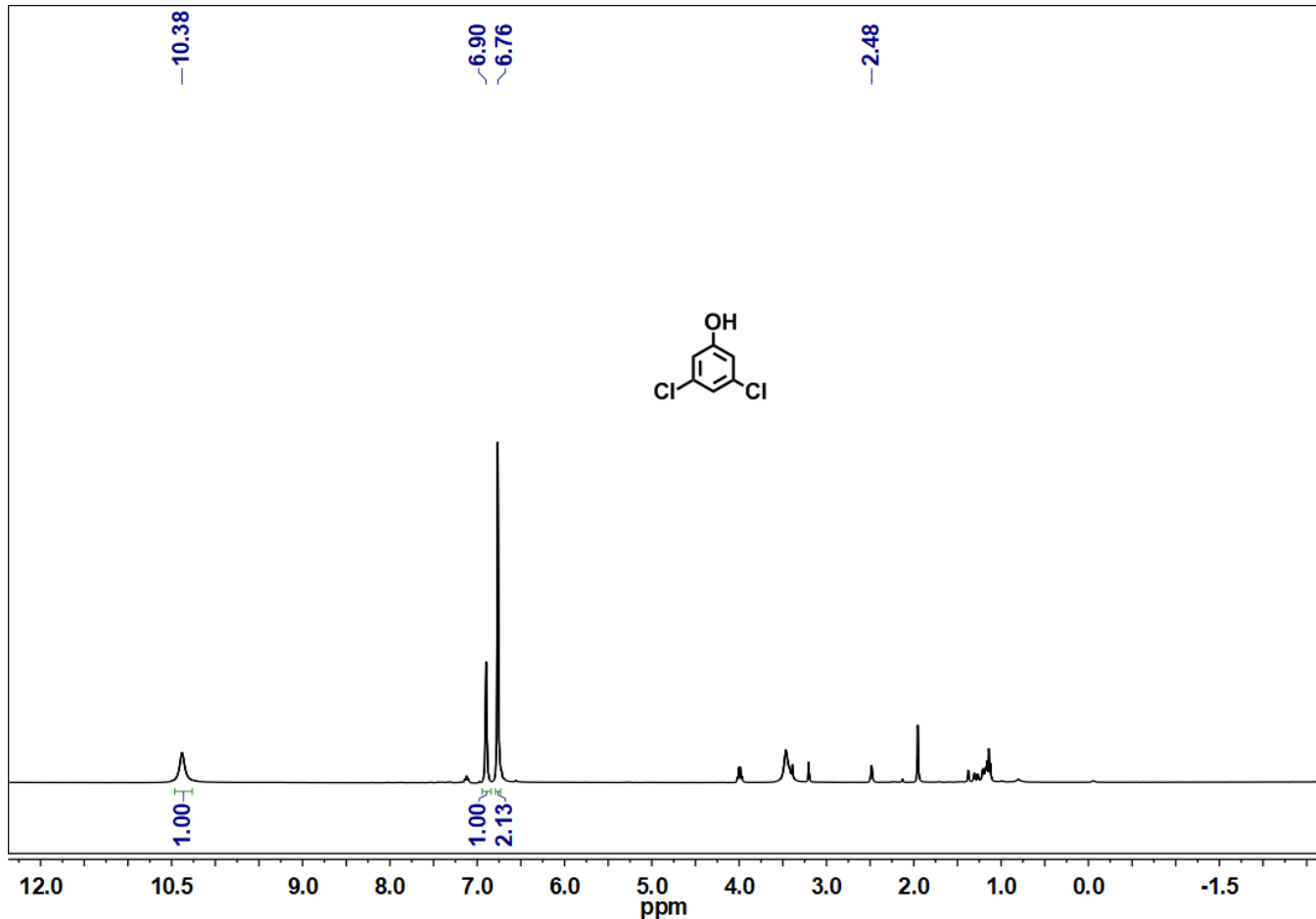

3,5-dichlorophenol (5e) ${ }^{1} \mathrm{H}$ NMR (400 MHz, DMSO-d 6 ) $\delta 10.38(\mathrm{~s}, 1 \mathrm{H}), 6.90(\mathrm{~s}, 1 \mathrm{H})$, $6.76(\mathrm{~s}, 2 \mathrm{H})$. 


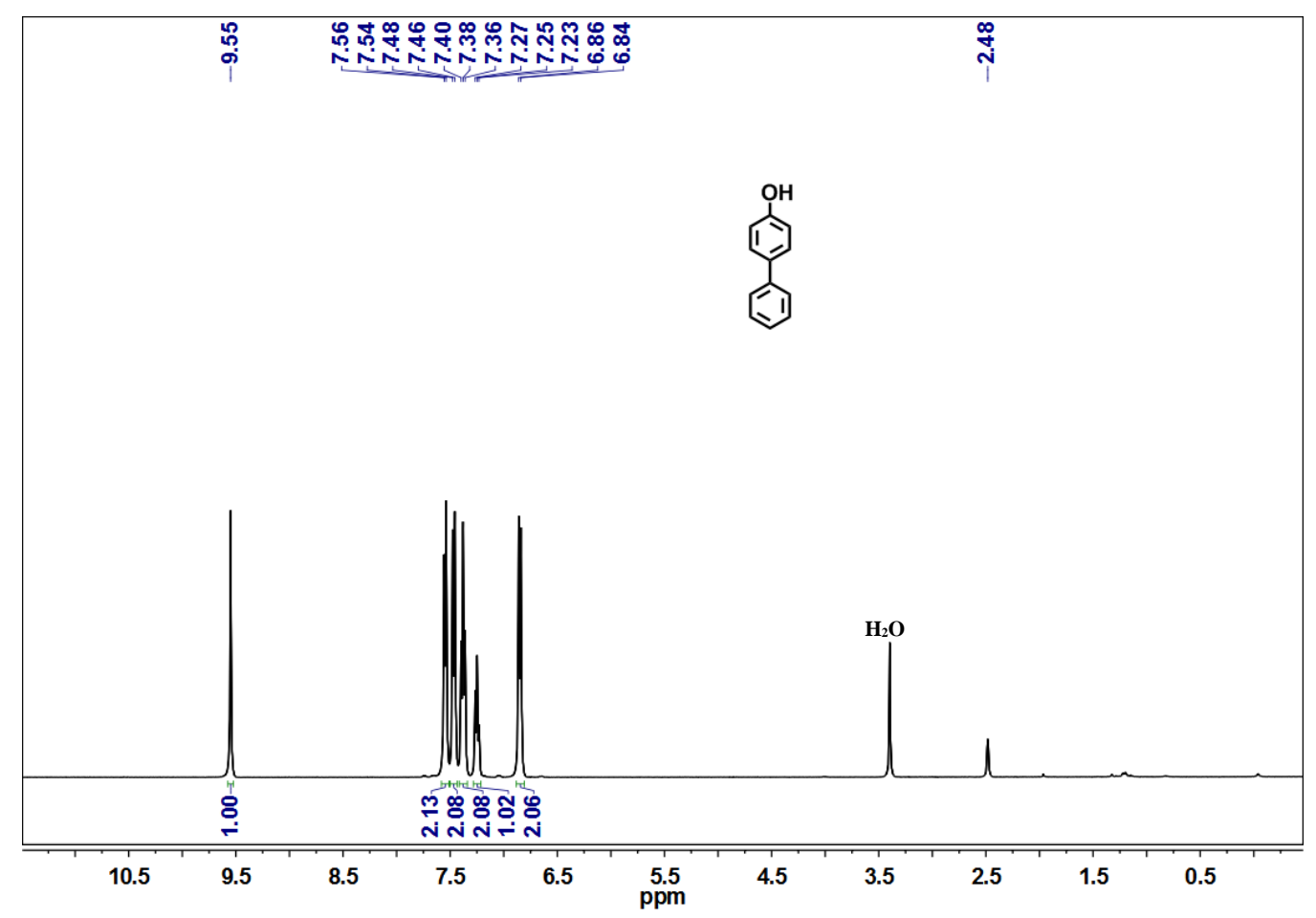

4-Phenylphenol (5f) ${ }^{1} \mathrm{H}$ NMR (400 MHz, DMSO- $\left.d_{6}\right) \delta 9.55$ (s, 1H), 7.56-7.54 (d, $J=$ $7.6 \mathrm{~Hz}, 2 \mathrm{H}), 7.48-7.46(\mathrm{~d}, J=8.1 \mathrm{~Hz}, 2 \mathrm{H}), 7.40-7.36(\mathrm{t}, 2 \mathrm{H}), 7.27-7.23(\mathrm{t}, 1 \mathrm{H}), 6.86-$ $6.84(\mathrm{~d}, J=8.1 \mathrm{~Hz}, 2 \mathrm{H})$.

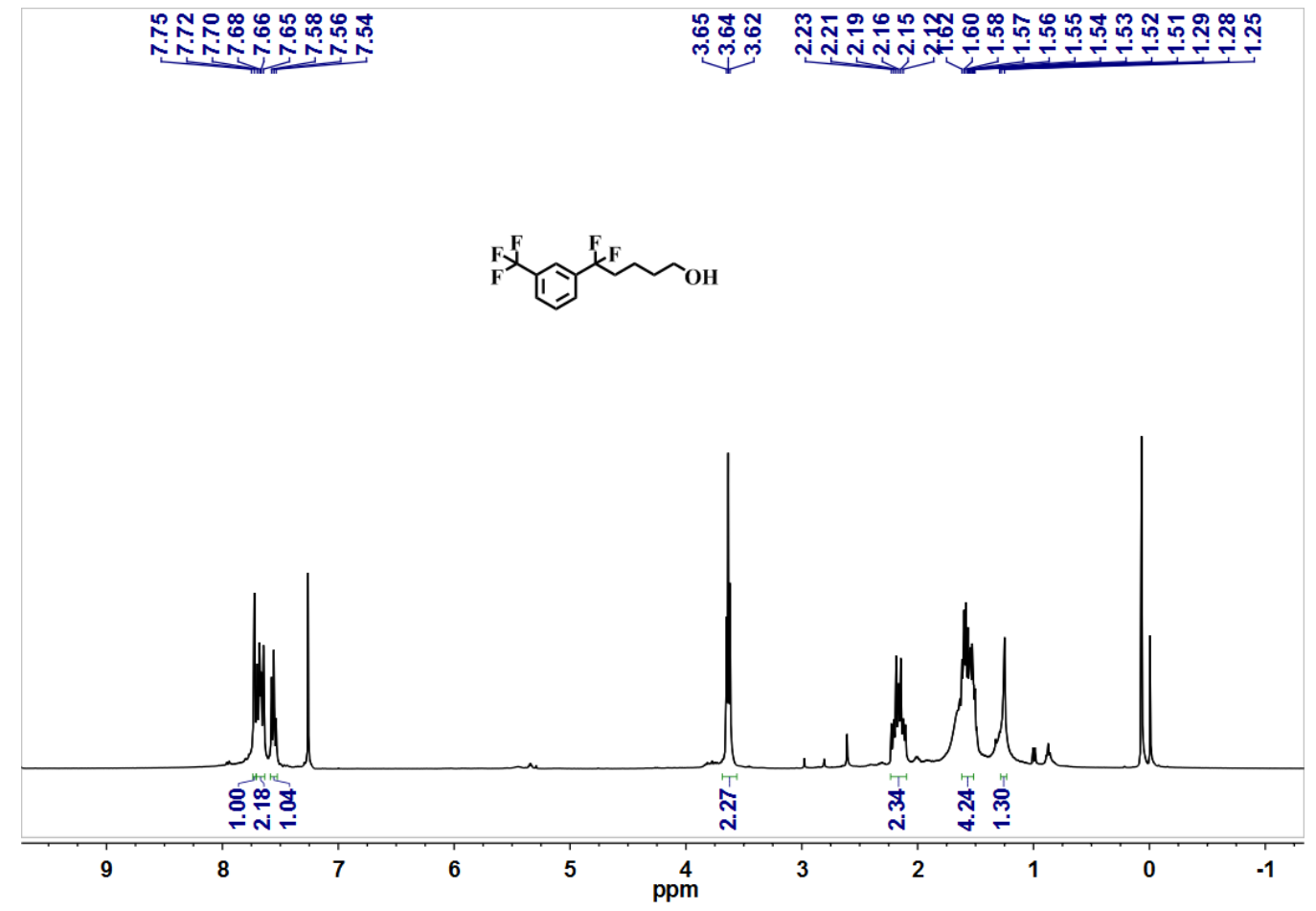




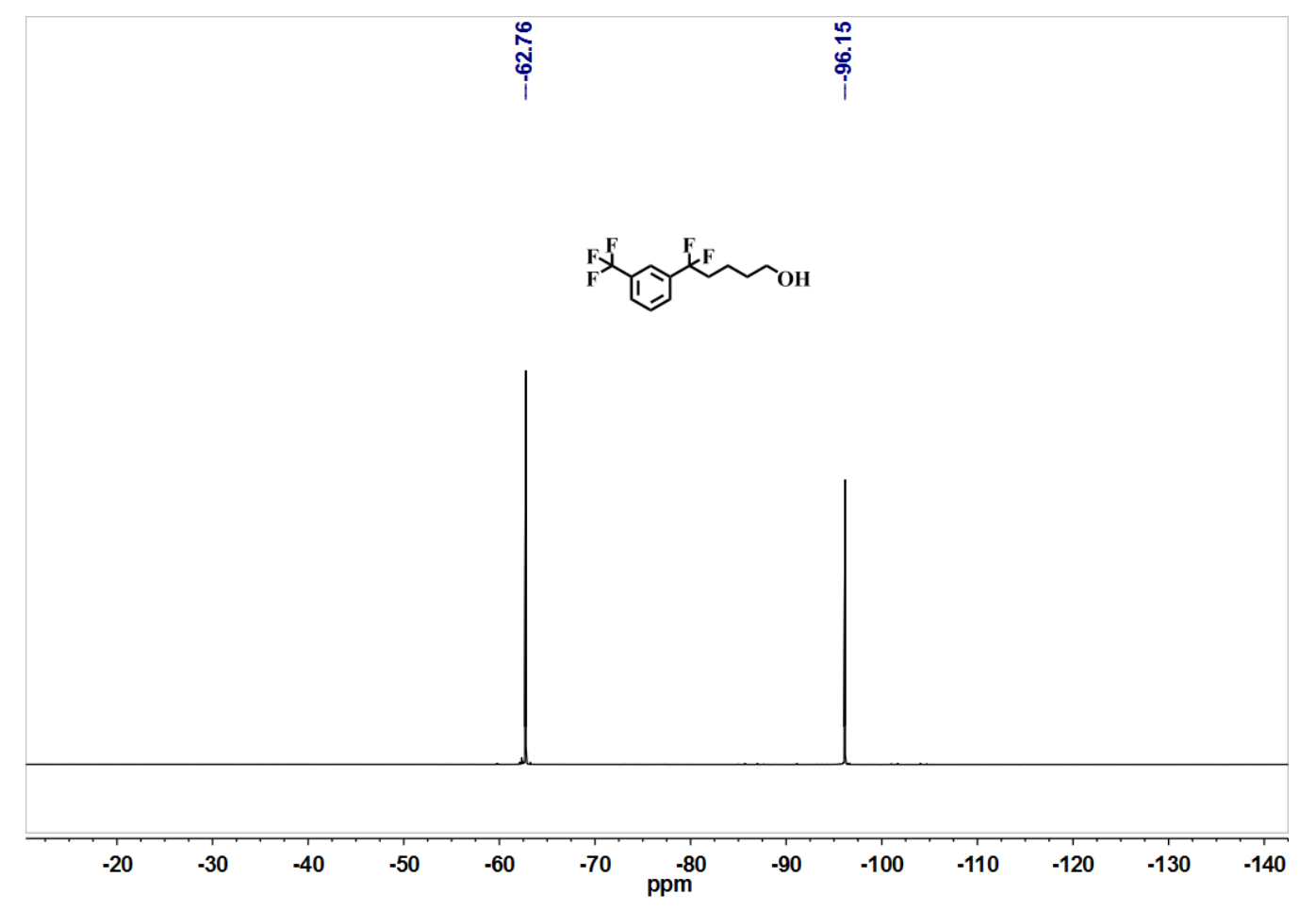

5,5-difluoro-5-(3-(trifluoromethyl)phenyl)pentan-1-ol (8a) ${ }^{1} \mathrm{H} \mathrm{NMR}\left(400 \mathrm{MHz}, \mathrm{CDCl}_{3}\right)$ $\delta 7.75(\mathrm{~s}, 1 \mathrm{H}), 7.72-7.65(\mathrm{~m}, 2 \mathrm{H}), 7.58-7.54(\mathrm{t}, 1 \mathrm{H}), 3.65-3.62(\mathrm{t}, 2 \mathrm{H}), 2.23-2.12(\mathrm{~m}$, $2 \mathrm{H}), 1.62-1.51(\mathrm{~m}, 4 \mathrm{H}), 1.29-1.25(\mathrm{t}, 1 \mathrm{H}) .{ }^{19} \mathrm{~F} \mathrm{NMR}\left(376 \mathrm{MHz}, \mathrm{CDCl}_{3}\right) \delta-62.76(\mathrm{~s}, 3 \mathrm{~F})$, $-96.15(\mathrm{~s}, 2 \mathrm{~F})$.

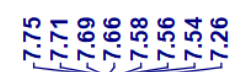

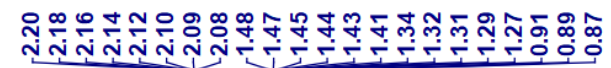

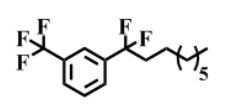

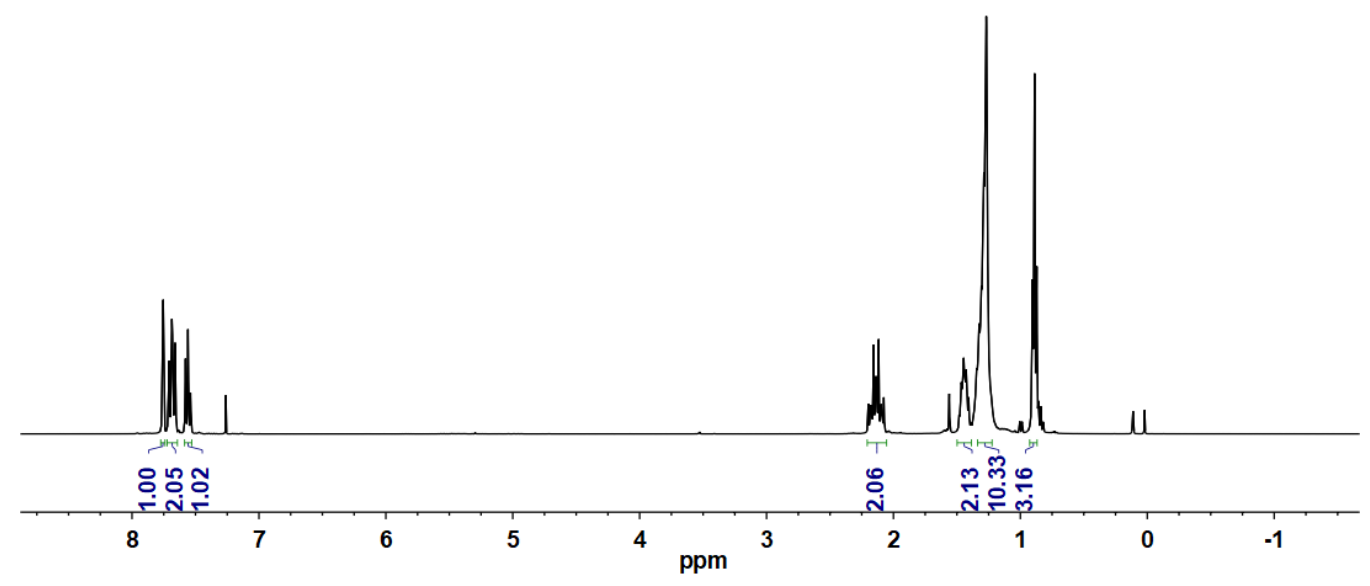




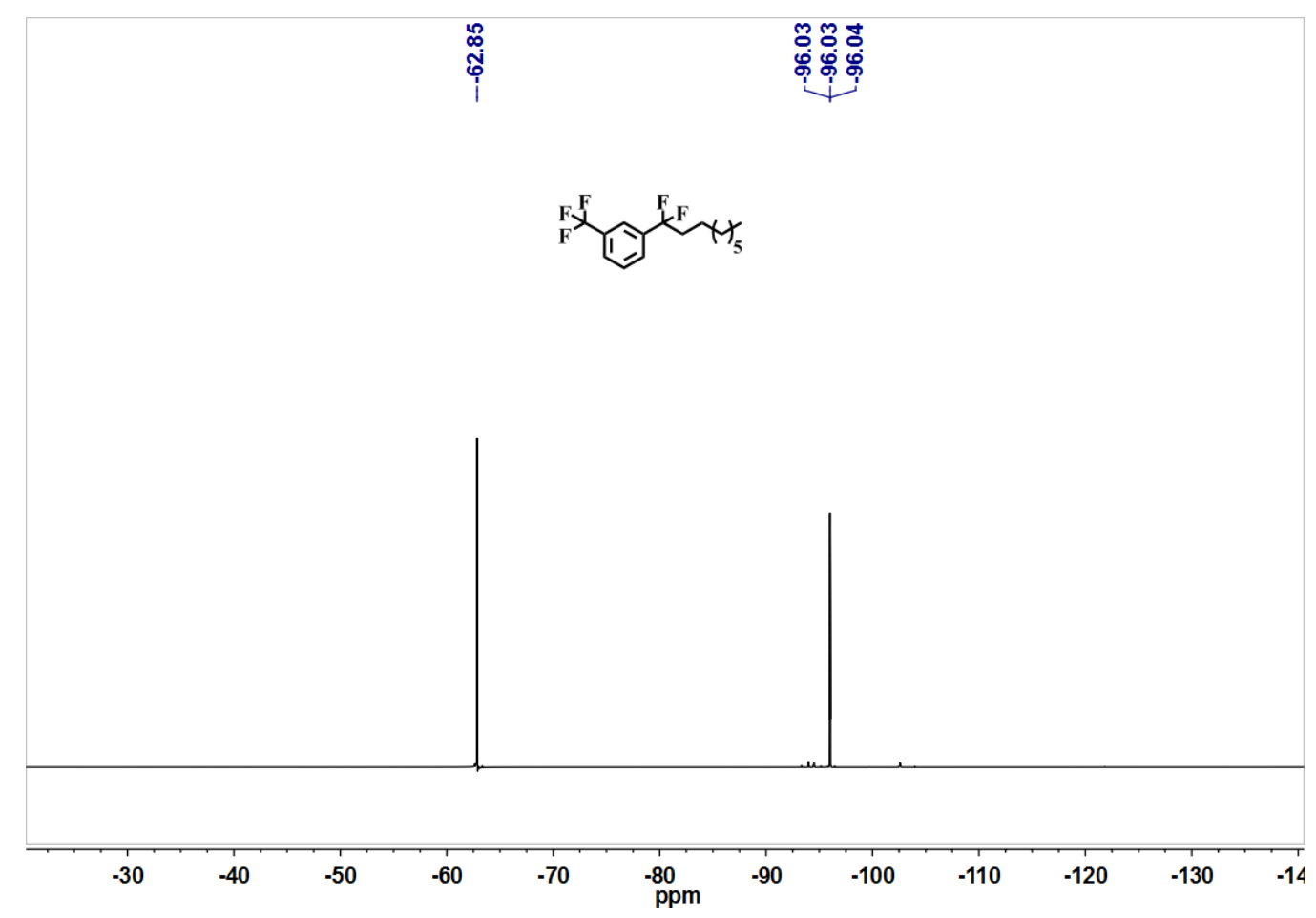

1-(1,1-difluorononyl)-3-(trifluoromethyl)benzene (8b) ${ }^{1} \mathrm{H}$ NMR $\left(400 \mathrm{MHz}, \mathrm{CDCl}_{3}\right) \delta$ $7.75(\mathrm{~s}, 1 \mathrm{H}), 7.71-7.66(\mathrm{t}, 2 \mathrm{H}), 7.58-7.54(\mathrm{t}, 1 \mathrm{H}), 2.20-2.08(\mathrm{~m}, 2 \mathrm{H}), 1.48-1.41(\mathrm{~m}, 2 \mathrm{H})$, 1.34-1.27 (m, 10H), 0.91-0.87 (t, 3H). $\left.{ }^{19} \mathrm{~F} \mathrm{NMR} \mathrm{(376} \mathrm{MHz,} \mathrm{CDCl}_{3}\right) \delta-62.85(\mathrm{~s}, 3 \mathrm{~F}),-$ $96.03(\mathrm{t}, 2 \mathrm{~F})$.

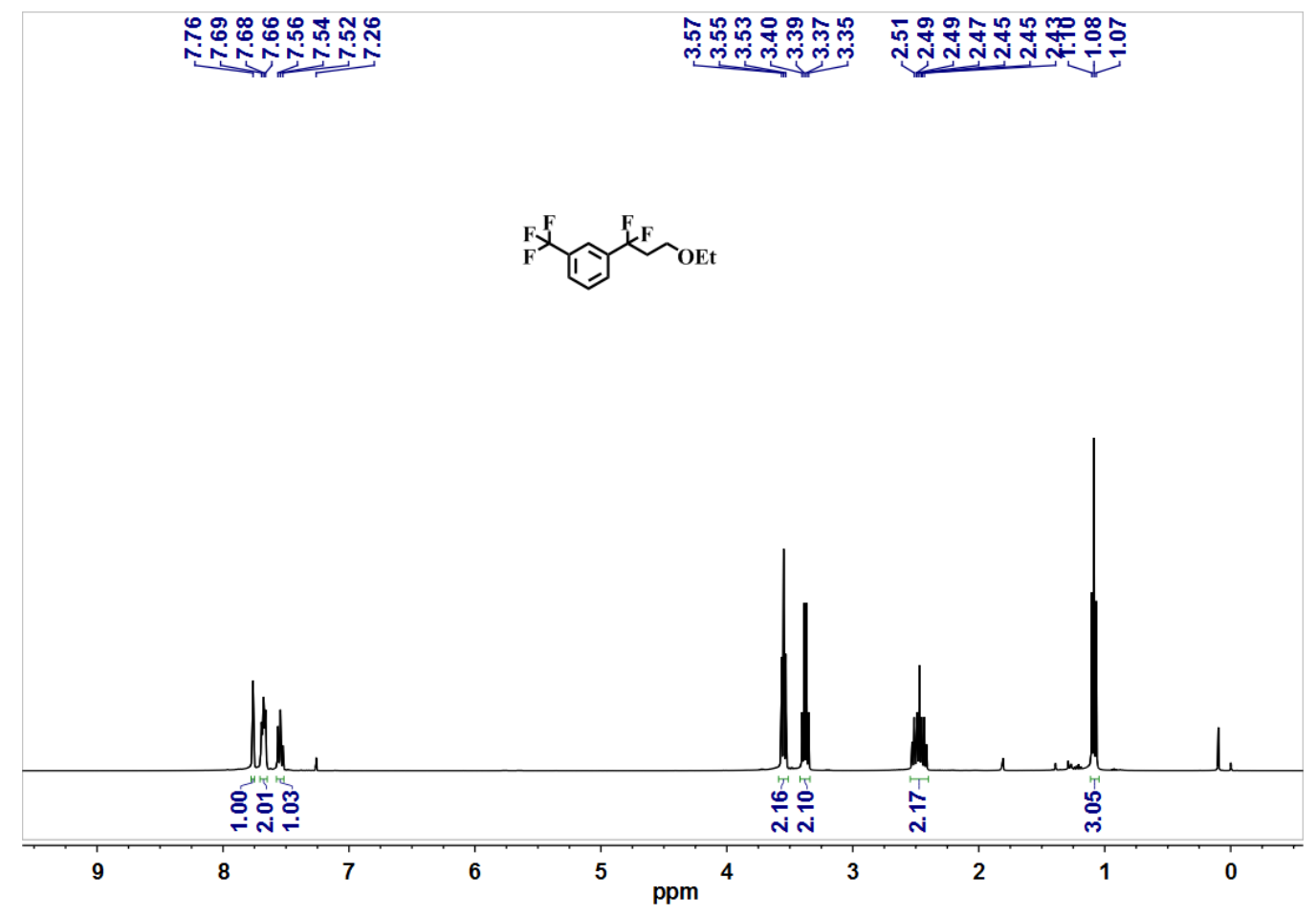




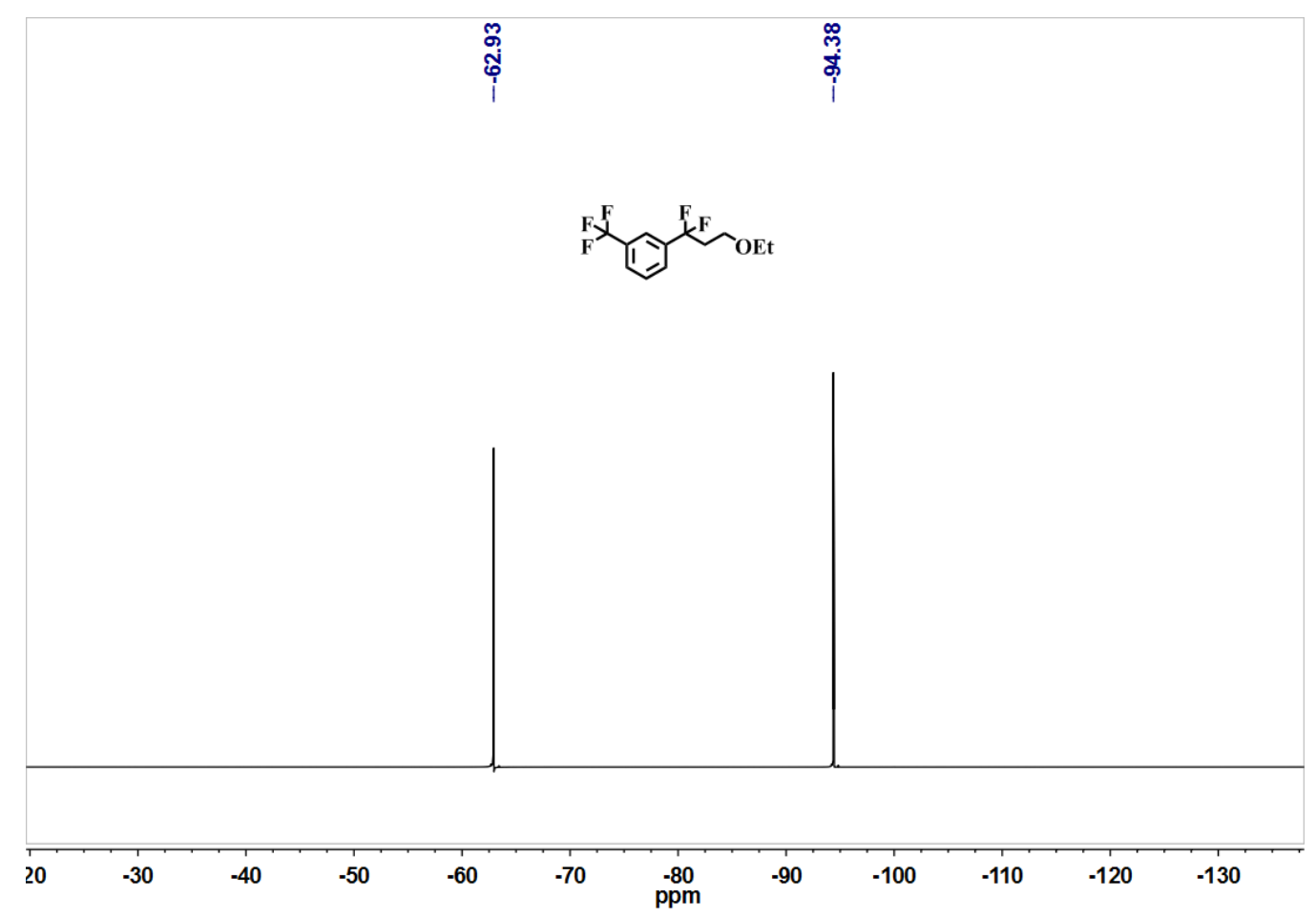

1-(3-ethoxy-1,1-difluoropropyl)-3-(trifluoromethyl)benzene (8c) ${ }^{1} \mathrm{H}$ NMR (400 MHz, $\left.\mathrm{CDCl}_{3}\right) \delta 7.76(\mathrm{~s}, 1 \mathrm{H}), 7.69-7.66(\mathrm{t}, 2 \mathrm{H}), 7.56-7.52(\mathrm{t}, 1 \mathrm{H}), 3.57-3.53(\mathrm{t}, 2 \mathrm{H}), 3.40-3.35$ (m, 2H), 2.51-2.43 (m, 2H), 1.10-1.07 (t, 3H). $\left.{ }^{19} \mathrm{~F} \mathrm{NMR} \mathrm{(376} \mathrm{MHz,} \mathrm{CDCl}_{3}\right) \delta-62.93(\mathrm{~s}$, 3F), -94.38 (s, 2F).

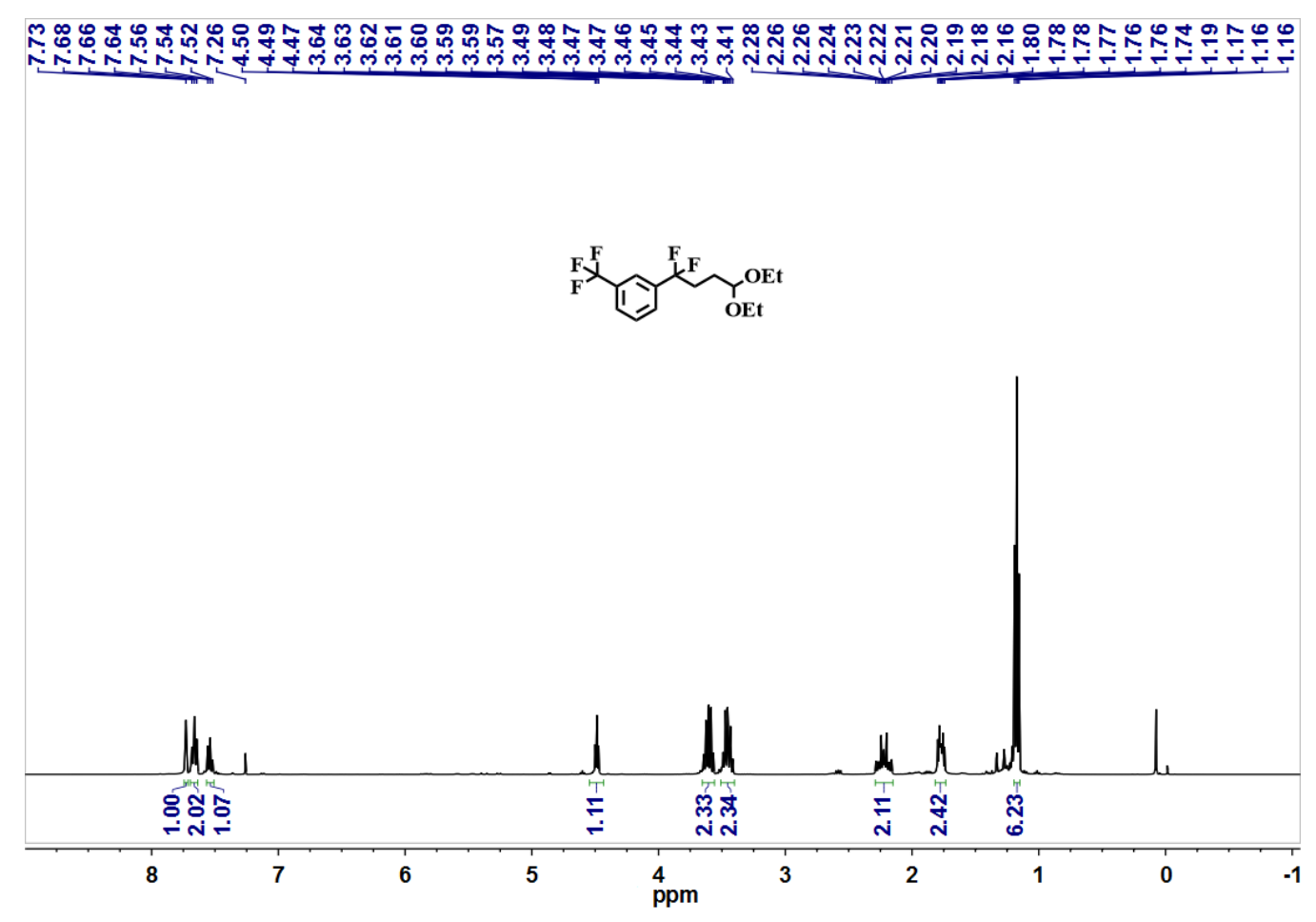




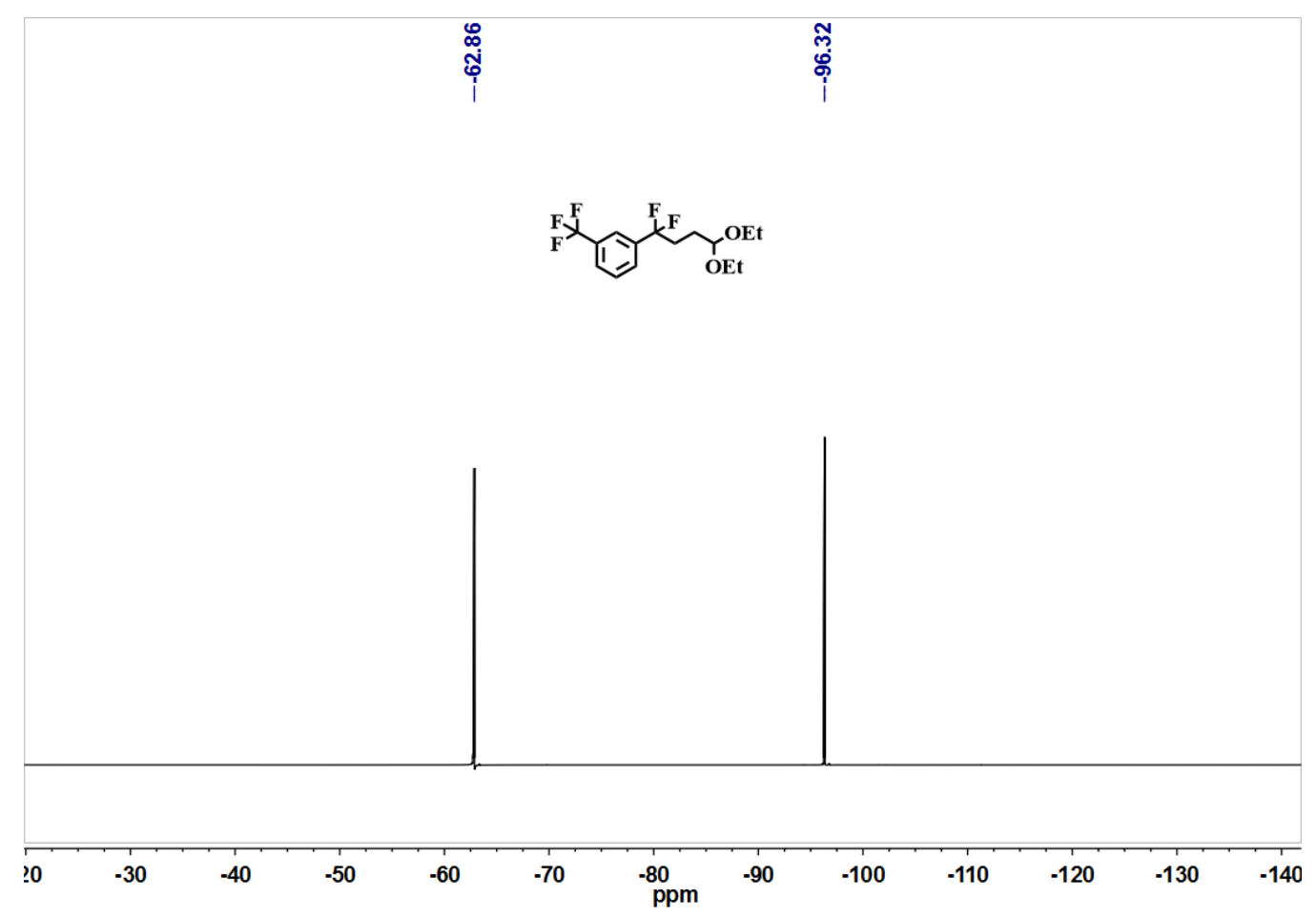

1-(5,5-diethoxy-1,1-difluoropentyl)-3-(trifluoromethyl)benzene (8d) ${ }^{1} \mathrm{H}$ NMR (400 $\left.\mathrm{MHz}, \mathrm{CDCl}_{3}\right) \delta 7.73(\mathrm{~s}, 1 \mathrm{H}), 7.68-7.64(\mathrm{t}, 2 \mathrm{H}), 7.56-7.52(\mathrm{t}, 1 \mathrm{H}), 4.50-4.47(\mathrm{t}, 1 \mathrm{H})$, 3.64-3.57 (m, 2H), 3.49-3.41 (m, 2H), 2.28-2.16 (m, 2H), 1.80-1.74 (m, 2H), 1.19-1.16 $(\mathrm{m}, 6 \mathrm{H}) .{ }^{19} \mathrm{~F} \mathrm{NMR}\left(376 \mathrm{MHz}, \mathrm{CDCl}_{3}\right) \delta-62.86(\mathrm{~s}, 3 \mathrm{~F}),-96.32(\mathrm{~s}, 2 \mathrm{~F})$.

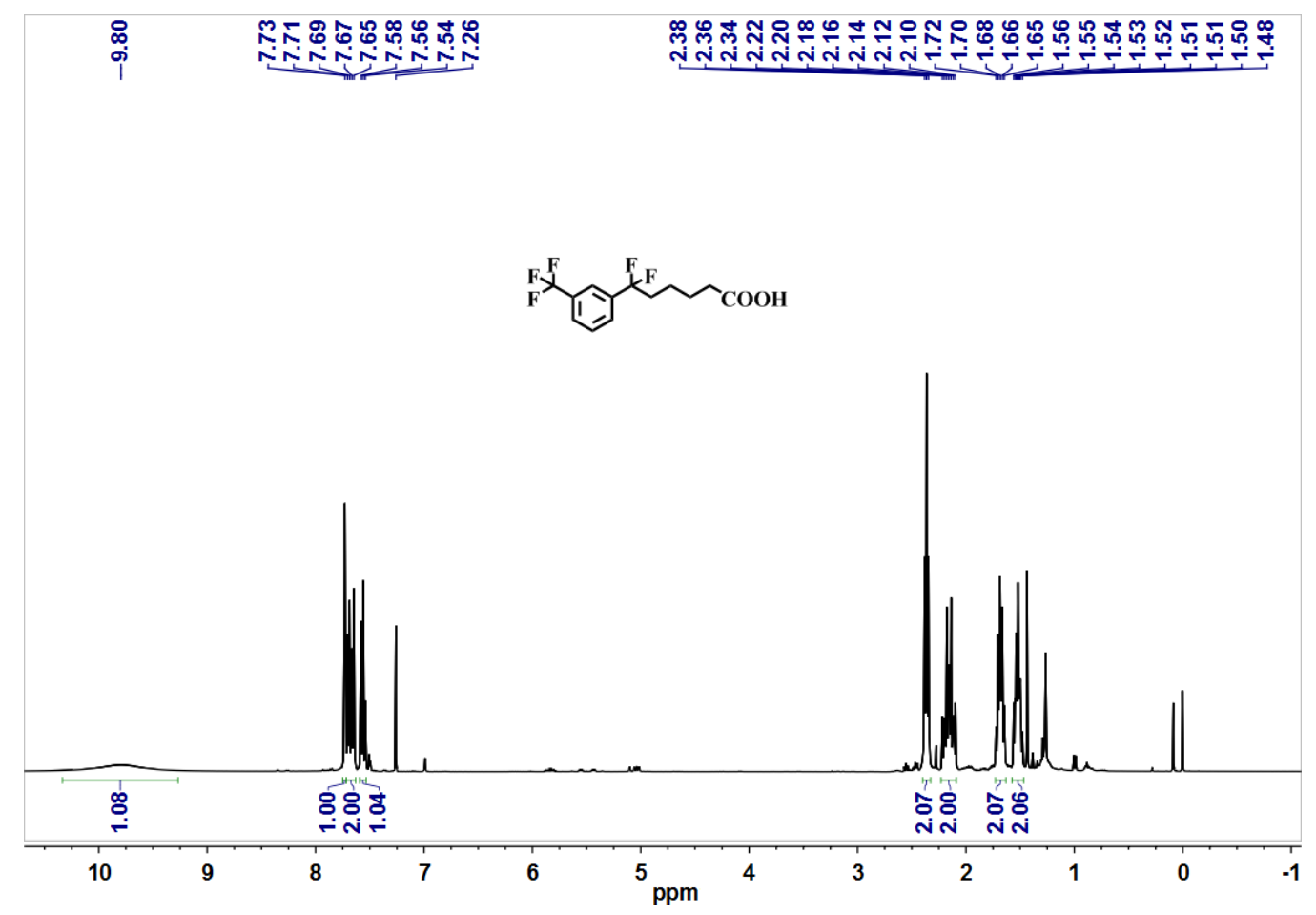




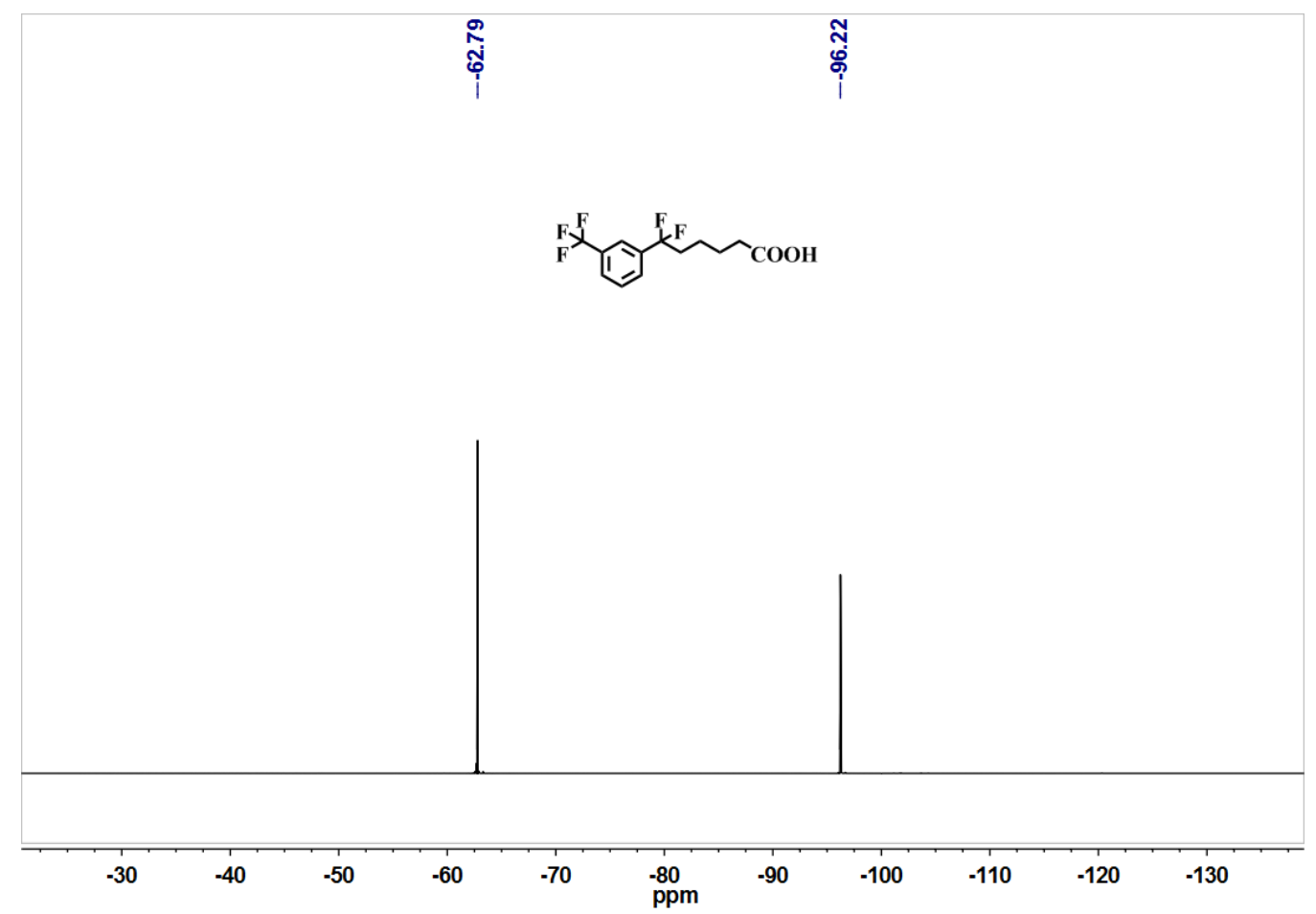

6,6-difluoro-6-(3-(trifluoromethyl)phenyl)hexanoic acid (8e) ${ }^{1} \mathrm{H}$ NMR (400 MHz, $\left.\mathrm{CDCl}_{3}\right) \delta 9.80(\mathrm{~s}, 1 \mathrm{H}), 7.73(\mathrm{~s}, 1 \mathrm{H}), 7.71-7.65(\mathrm{~m}, 2 \mathrm{H})$, 7.58-7.54 (t, 1H), 2.38-2.20 (m, 2H), 2.18-2.10 (m, 2H), 1.72-1.65 (m, 2H), 1.56-1.48 (m, 2H). ${ }^{19} \mathrm{~F}$ NMR (376 MHz, $\left.\mathrm{CDCl}_{3}\right) \delta-62.79(\mathrm{~s}, 3 \mathrm{~F}),-96.22(\mathrm{~s}, 2 \mathrm{~F})$.

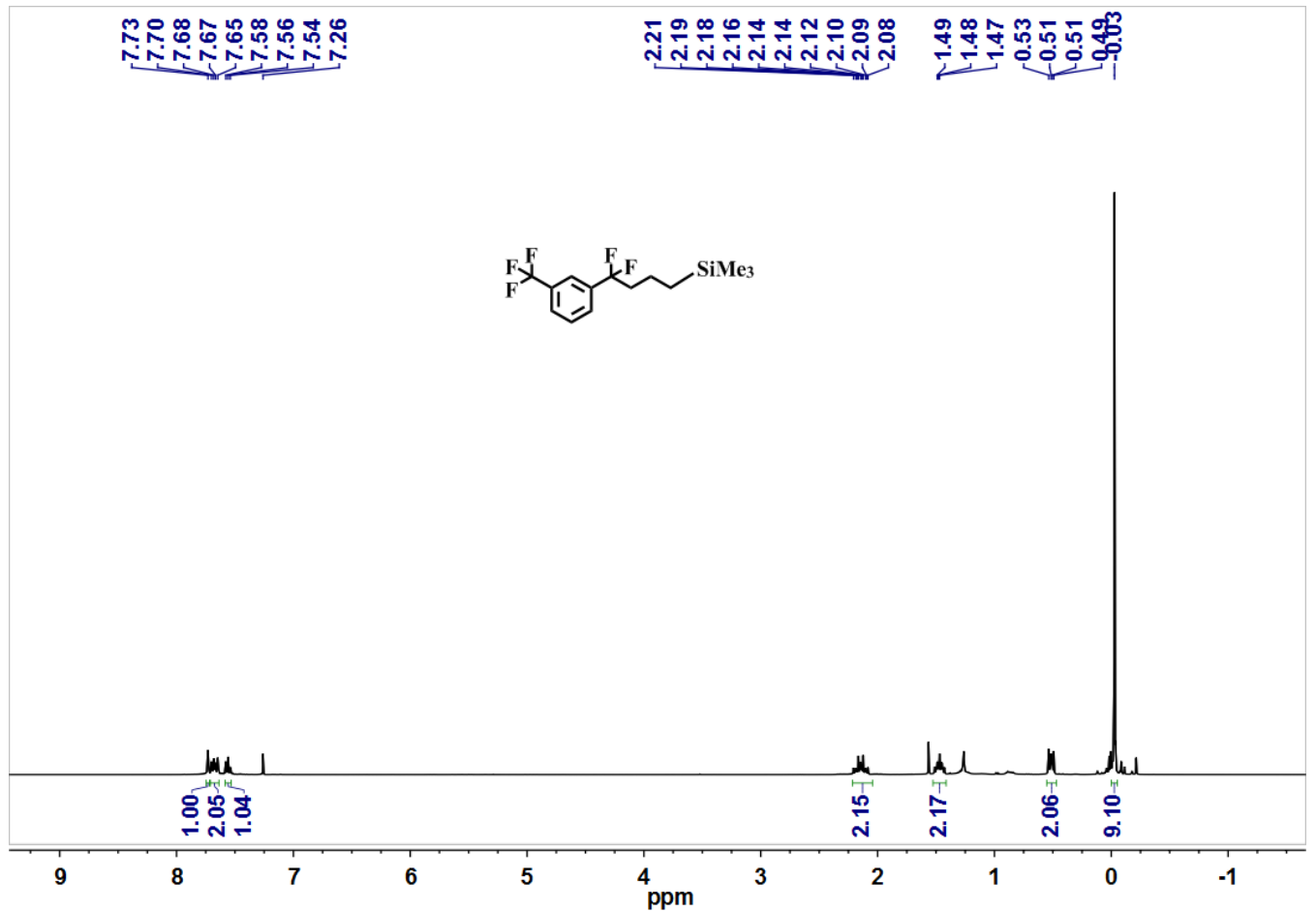




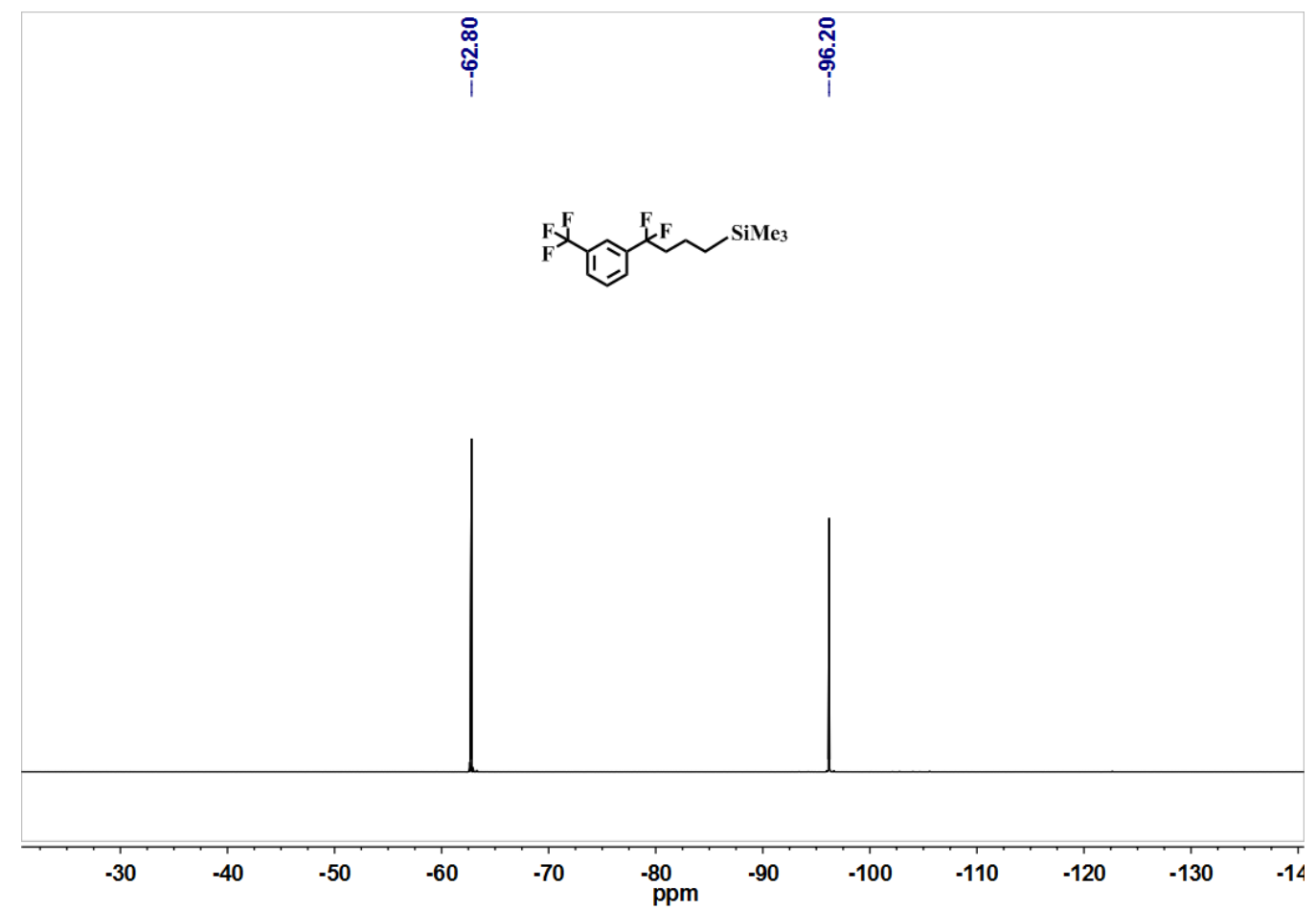

(4,4-difluoro-4-(3-(trifluoromethyl)phenyl)butyl)trimethylsilane (8f) ${ }^{1} \mathrm{H}$ NMR (400 $\left.\mathrm{MHz}, \mathrm{CDCl}_{3}\right) \delta 7.73(\mathrm{~s}, 1 \mathrm{H}), 7.70-7.65(\mathrm{~m}, 2 \mathrm{H}), 7.58-7.54(\mathrm{t}, 1 \mathrm{H}), 2.21-2.08(\mathrm{~m}, 2 \mathrm{H})$, 1.49-1.47 (m, 2H), 0.53-0.49 (m, 2H), $0.03(\mathrm{~s}, 9 \mathrm{H}) .{ }^{19} \mathrm{~F}$ NMR (376 MHz, $\left.\mathrm{CDCl}_{3}\right) \delta$ $62.80(\mathrm{~s}, 3 \mathrm{~F}),-96.20$ (s, 2F).

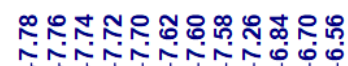

rertivinitio

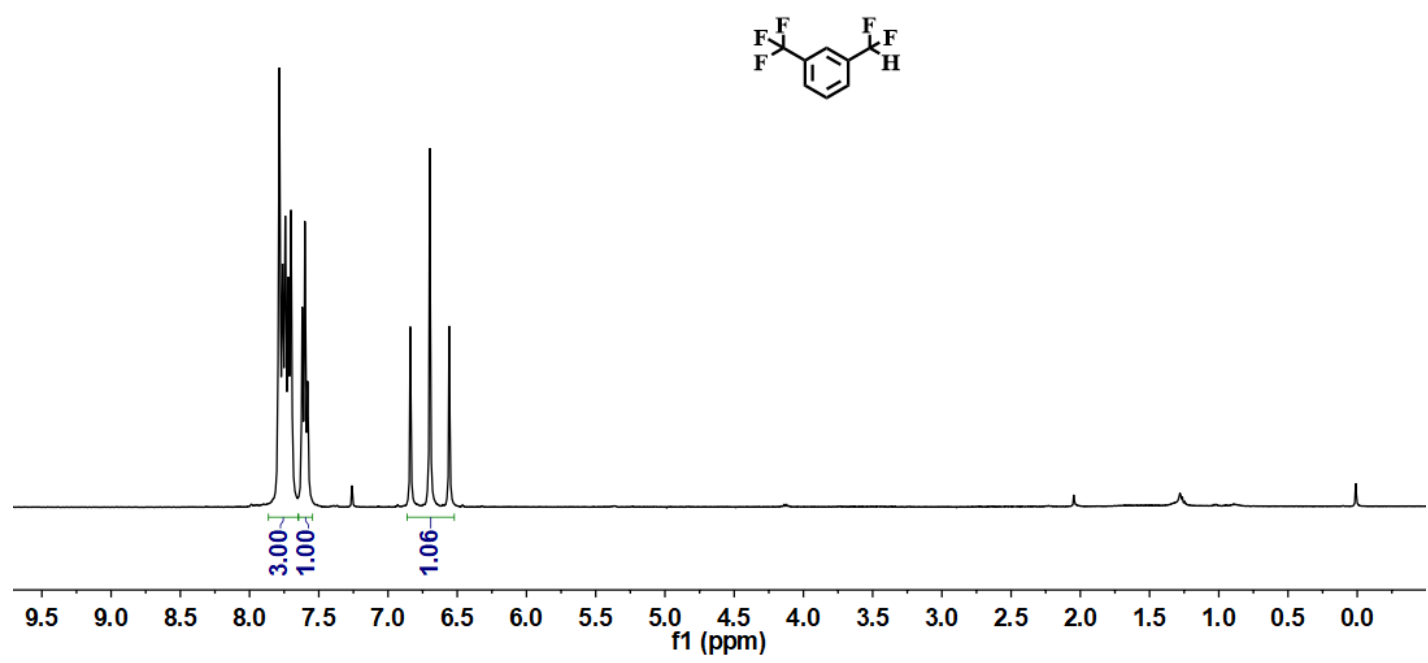



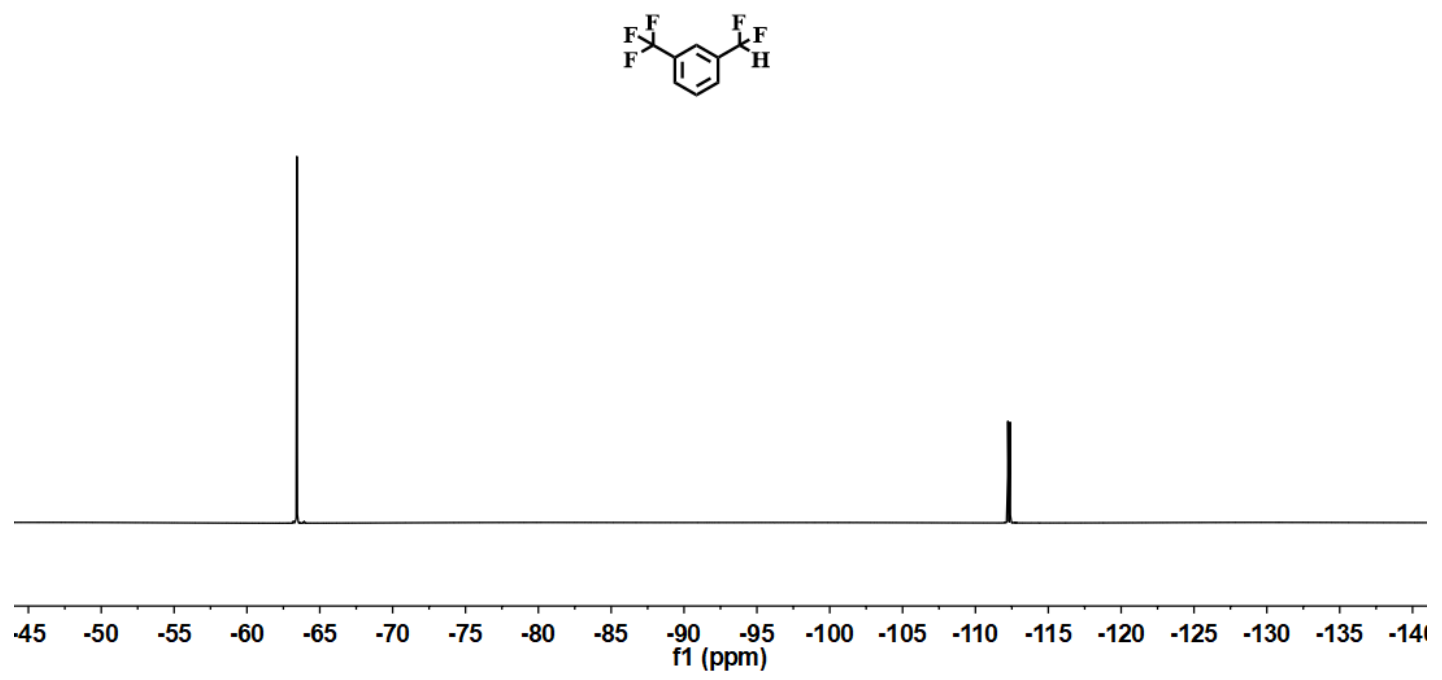

1-(difluoromethyl)-3-(trifluoromethyl)benzene (12) ${ }^{1} \mathrm{H}$ NMR (400 $\left.\mathrm{MHz}, \mathrm{CDCl}_{3}\right) \delta$ 7.78-7.70 (m, 3H), 7.62-7.58 (t, 1H), $6.84-6.56(\mathrm{t}, 1 \mathrm{H}) .{ }^{19} \mathrm{~F} \mathrm{NMR}\left(376 \mathrm{MHz}, \mathrm{CDCl}_{3}\right) \delta$ $-63.43(\mathrm{~s}, 3 \mathrm{~F}),-112.23(\mathrm{~s}, 1 \mathrm{~F}),-112.38(\mathrm{~s}, 1 \mathrm{~F})$.

\section{References}

1. Bettelheim, A.; White, B. A.; Raybuck, S. A.; Murray, R. W. Inorg. Chem. 1987, 26, 1009-1017.

2. Lin, S.; Diercks, C. S.; Zhang, Y.-B.; Kornienko, N.; Nichols, E. M.; Zhao, Y.; Paris, A. R.; Kim, D.; Yang, P.; Yaghi, O. M.; Chang, C. J. Science, 2015, 349, 1208-1213. 